Florida International University FIU Digital Commons

$5-17-2012$

\title{
Dynamic Modeling and Analysis of Single-Stage Boost Inverters under Normal and Abnormal Conditions
}

\author{
Ali Kashefi Kaviani \\ Florida International University, akash002@fiu.edu
}

DOI: $10.25148 /$ etd.FI12071107

Follow this and additional works at: https://digitalcommons.fiu.edu/etd

\section{Recommended Citation}

Kashefi Kaviani, Ali, "Dynamic Modeling and Analysis of Single-Stage Boost Inverters under Normal and Abnormal Conditions" (2012). FIU Electronic Theses and Dissertations. 655.

https://digitalcommons.fiu.edu/etd/655 


\title{
FLORIDA INTERNATIONAL UNIVERSITY
}

Miami, Florida

\section{DYNAMIC MODELING AND ANALYSIS OF SINGLE-STAGE BOOST INVERTERS UNDER NORMAL AND ABNORMAL CONDITIONS}

\author{
A dissertation submitted in partial fulfillment of \\ the requirements for the degree of \\ DOCTOR OF PHILOSOPHY \\ in \\ ELECTRICAL ENGINEERING \\ by \\ Ali Kashefi Kaviani
}


To: Dean Amir Mirmiran

College of Engineering and Computing

This dissertation, written by Ali Kashefi Kaviani, and entitled Dynamic Modeling and Analysis of Single-Stage Boost Inverters under Normal and Abnormal Conditions, having been approved in respect to style and intellectual content, is referred to you for judgment.

We have read this dissertation and recommend that it be approved.

$\begin{array}{r}\hline \text { Nezih Pala } \\ \hline \text { Jean Andrian } \\ \hline \text { Yimin Zhu } \\ \hline \text { Chris Edrington } \\ \hline \text { Kehrooz Mirafzal, Co-Major Professor } \\ \hline\end{array}$

Date of Defense: May 17, 2012

The dissertation of Ali Kashefi Kaviani is approved.

Dean Amir Mirmiran

College of Engineering and Computing

Dean Lakshmi N. Reddi

University Graduate School

Florida International University, 2012 
(C) Copyright 2012 by Ali Kashefi Kaviani

All rights reserved. 


\section{DEDICATION}

To My Dear Parents ... 


\section{ACKNOWLEDGMENTS}

I would like to express my heart-felt appreciation to all who contributed in many ways to success of my $\mathrm{PhD}$ study and completion of this dissertation. I am especially grateful to my advisor, Dr. Behrooz Mirafzal for his belief in my abilities and for accepting me in his laboratory. I appreciate his valuable guidance and contribution to this accomplishment. I would like to extend my sincerest thanks to my major-professor Dr. Kang Yen for his excellent supervision and his kind understanding during the hardest times of my life at FIU. I am indebted to Dean Amir Mirmiran, and the Department of Electrical and Computer Engineer for several semesters of financial support. Obviously, I would not be able to study at FIU without their assistance. I warmly thank our graduate program director, Dr. Jean Andrian, and the chairperson Dr. Shekhar Bhansali, for their endless supports. I wish to also thank the members of my committee, Dr. Nezih Pala, Dr. Yimin Zhu, and Dr. Chris Edrington, for their support, patience and advice. I should also acknowledge the dissertation year fellowship from the FIU graduate school during Fall 2011 and Spring 2012.

Last but not least, I express my deepest gratitude to all my dear friends, who helped me to make some of best years of life in Miami, and my special thanks go to Mr. Brian Hadley for his great help in writing this dissertation. 


\title{
ABSTRACT OF THE DISSERTATION \\ DYNAMIC MODELING AND ANALYSIS OF SINGLE-STAGE BOOST \\ INVERTERS UNDER NORMAL AND ABNORMAL CONDITIONS
}

\author{
by
}

Ali Kashefi Kaviani

Florida International University, 2012

Miami, Florida

\section{Professor Behrooz Mirafzal and Professor Kang Yen, Co-Major Professors}

Inverters play key roles in connecting sustainable energy (SE) sources to the local loads and the ac grid. Although there has been a rapid expansion in the use of renewable sources in recent years, fundamental research, on the design of inverters that are specialized for use in these systems, is still needed. Recent advances in power electronics have led to proposing new topologies and switching patterns for single-stage power conversion, which are appropriate for SE sources and energy storage devices. The current source inverter (CSI) topology, along with a newly proposed switching pattern, is capable of converting the low dc voltage to the line ac in only one stage. Simple implementation and high reliability, together with the potential advantages of higher efficiency and lower cost, turns the so-called, single-stage boost inverter (SSBI), into a viable competitor to the existing SE-based power conversion technologies.

The dynamic model is one of the most essential requirements for performance analysis and control design of any engineering system. Thus, in order to have satisfactory operation, it is necessary to derive a dynamic model for the SSBI system. However, 
because of the switching behavior and nonlinear elements involved, analysis of the SSBI is a complicated task.

This research applies the state-space averaging technique to the SSBI to develop the state-space-averaged model of the SSBI under stand-alone and grid-connected modes of operation. Then, a small-signal model is derived by means of the perturbation and linearization method. An experimental hardware set-up, including a laboratory-scaled prototype SSBI, is built and the validity of the obtained models is verified through simulation and experiments. Finally, an eigenvalue sensitivity analysis is performed to investigate the stability and dynamic behavior of the SSBI system over a typical range of operation. 


\section{TABLE OF CONTENTS}

CHAPTER

PAGE

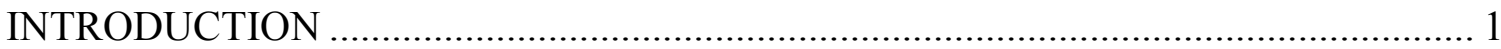

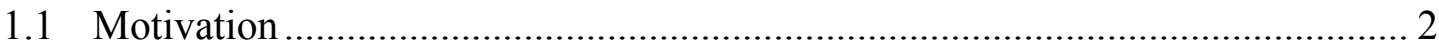

1.2 Problem Statement and Research Objectives .................................................. 4

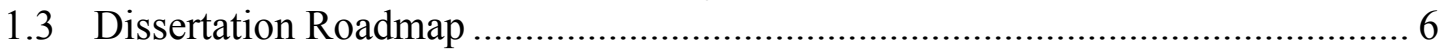

DC-AC CONVERSION IN DISTRIBUTED GENERATION SYSTEMS ........................ 8

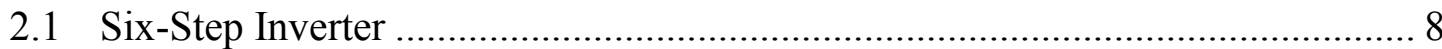

2.2 Pulse-Width-Modulated Inverter...................................................................... 10

2.2.1 Sinusoidal Pulse-Width-Modulation (SPWM) ........................................... 11

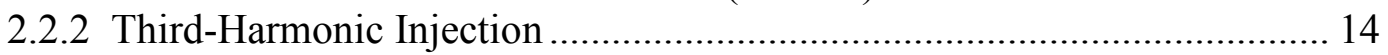

2.2.3 Selective Harmonic Elimination.............................................................. 14

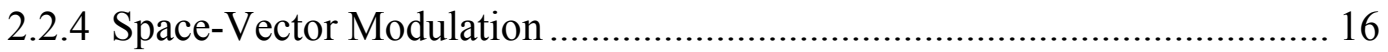

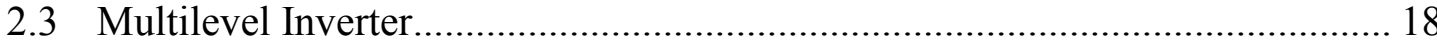

2.3.1 Diode-Clamped Multilevel Inverter (DCMI) .......................................... 19

2.3.2 Flying-Capacitor Multilevel Inverter (FCMI) …….................................. 22

2.3.3 Cascaded-Inverters with Separate dc-Sources ............................................ 23

2.3.4 A Comparison among the Multilevel Inverters ........................................ 23

2.4 DC-DC Converters for DG Applications …………......................................... 24

2.4.1 Basic Single-Stage Conversion System ................................................ 25

2.4.2 Conventional Two-Stage Conversion System .......................................... 26

2.4.3 Multilevel Inverter ............................................................................ 27

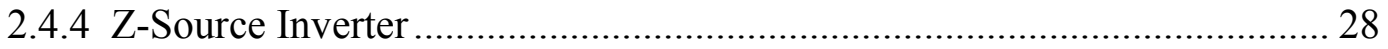

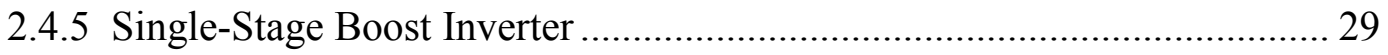

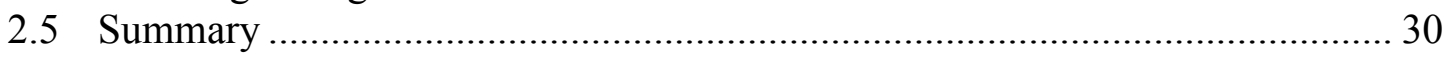

MODELING OF POWER ELECTRONIC CONVERTERS ………………………....... 32

3.1 Steady-State Modeling of DC-DC Converters .................................................... 32

3.2 Steady-State Modeling of DC-AC Converters..................................................... 34

3.2.1 Steady-State Model of SPWM Inverter ...................................................... 34

3.2.2 Steady-State Model of SVPWM Inverter ..................................................... 36

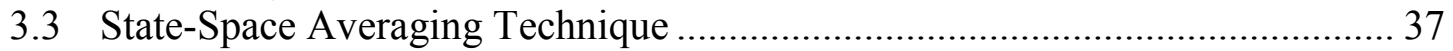

3.4 Dynamic Modeling of DC-DC Converters ………………………………........ 39

3.4.1. Linear Equivalent Circuits for Nonlinear PWM Converters ...................... 42

3.4.2 Linear Equivalent Circuits for Switch-Diode Combinations....................... 43

3.4.3 Modeling under Non-Small Ripple Conditions .......................................... 44

3.4.4 Converter Analysis in Discontinuous Modes ............................................ 45

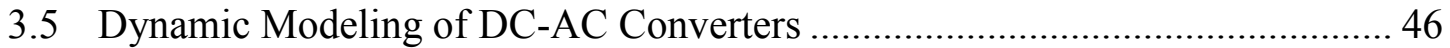

3.5.1 General Methodology for Modeling Inverters .......................................... 47

3.5.2 Transformer-Based Equivalent Circuits ..................................................... 49

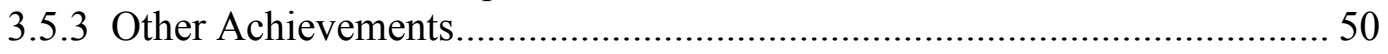

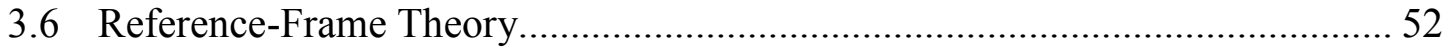




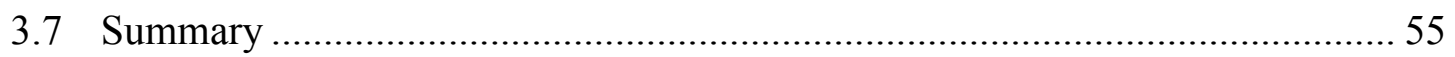

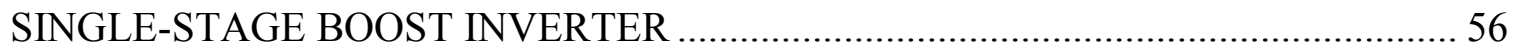

4.1 Three-Phase Single-Stage Boost Inverter …………….................................... 56

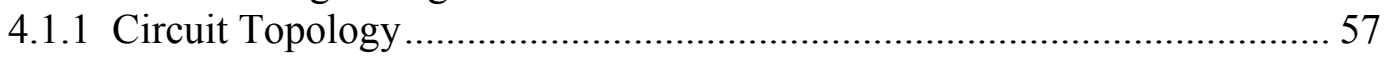

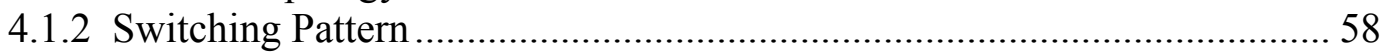

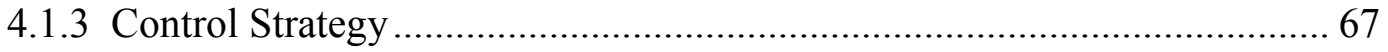

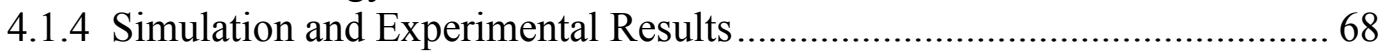

4.1.5 Abnormal Conditions.............................................................................. 73

4.2 Single-Phase Single-Stage Boost Inverter...................................................... 79

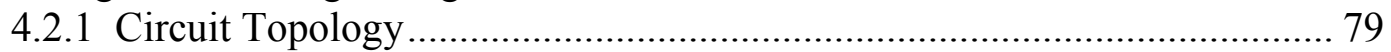

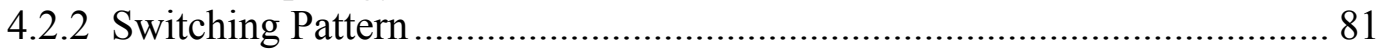

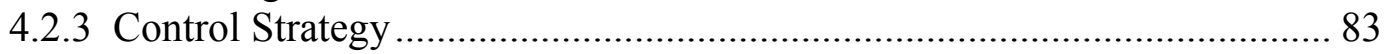

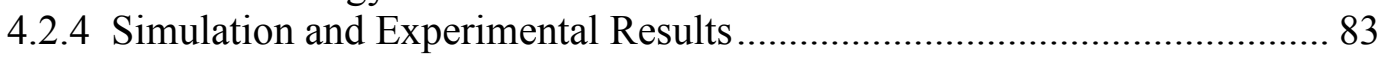

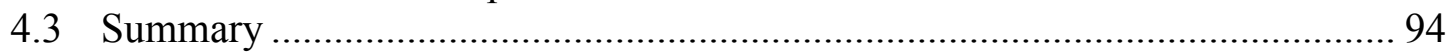

STATE-SPACE-AVERAGED MODEL OF THE STAND-ALONE SINGLE-STAGE

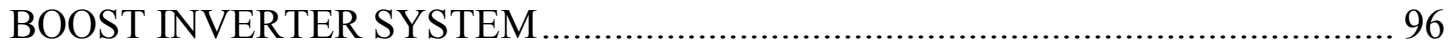

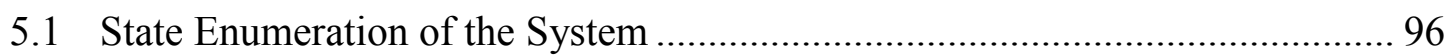

5.2 State-Space-Averaged Model of the SSBI .................................................. 99

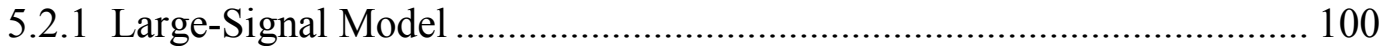

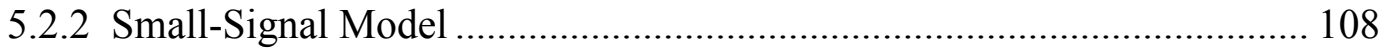

5.3 Eigenvalue Sensitivity Analysis.................................................................... 109

5.4 Simulation and Experimental Results ……………….................................. 117

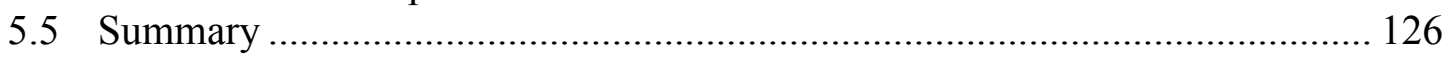

STATE-SPACE-AVERAGED MODEL OF THE GRID-CONNECTED SINGLE-

STAGE BOOST INVERTER SYSTEM ......................................................... 128

6.1 State Enumeration of the System .............................................................. 128

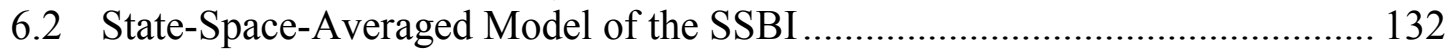

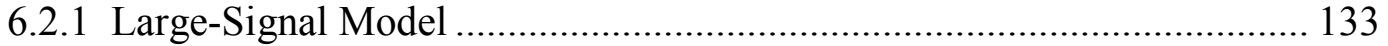

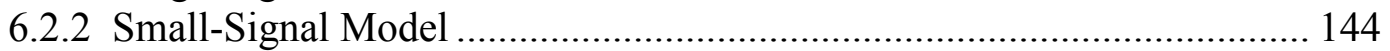

6.3 Eigenvalue Sensitivity Analysis................................................................ 145

6.4 Simulation and Experimental Results ………………................................... 153

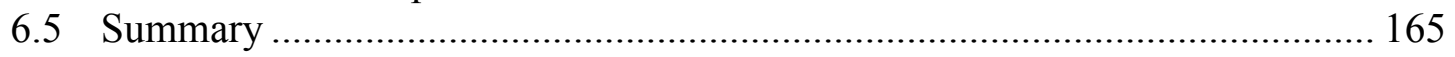

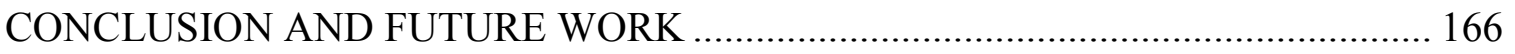

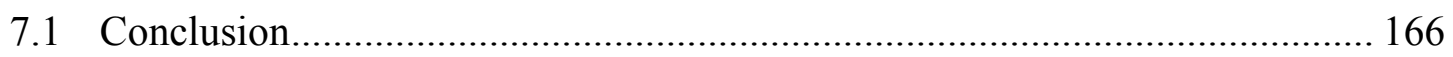

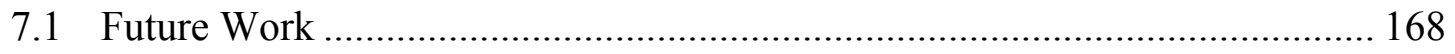

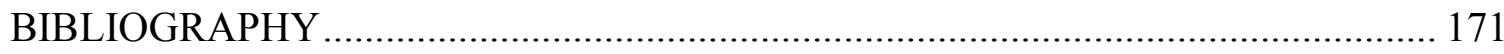

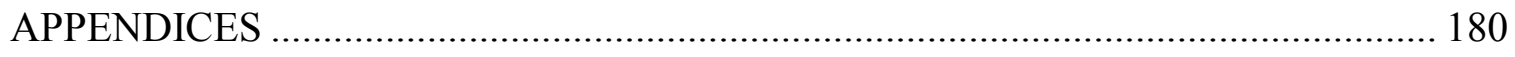

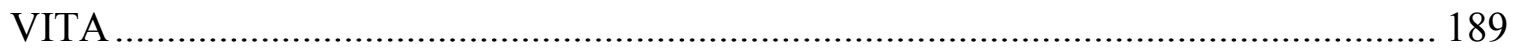




\section{LIST OF TABLES}

TABLE

PAGE

Table 2.1: Switching table and output voltages of a three-phase inverter ...................... 17

Table 2.2: Switching table of a three-level diode-clamped inverter ............................... 19

Table 2.3: Comparison of power components, required per phase leg, among the multilevel inverters

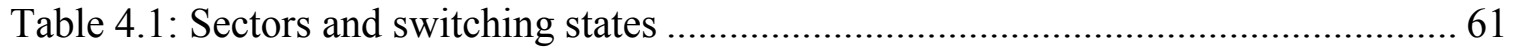

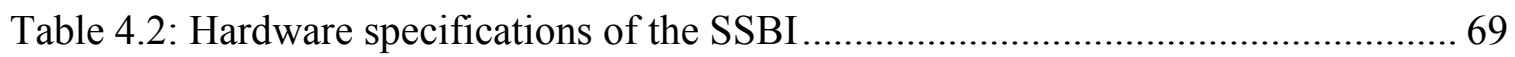

Table 4.3: Operating points of SSBI for nonlinear and linear loads............................ 70

Table 4.4: On/off time-intervals of the switches during each switching cycle................ 81

Table 4.5: Proposed switching table during each switching cycle, Ts ......................... 82

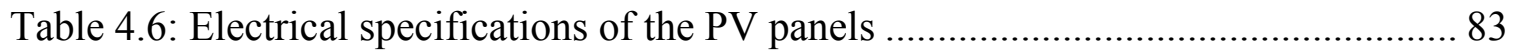

Table 4.7: The circuit parameters of the simulation and hardware setup ....................... 85

Table 4.8: Performance of the single-phase SSBI for different PV panels at maximum efficiency and minimum THD ................................................................. 93

Table 5.1: The possible states of the stand-alone SSBI system .................................. 98

Table 5.2: Circuit diagrams and state-space equations of the SSBI under CCM, for Sector

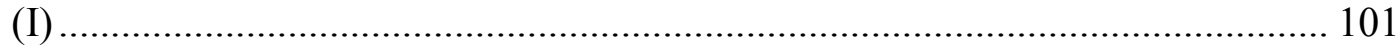

Table 5.3: Averaged system matrices of the stand-alone SSBI system for all sectors ... 102

Table 5.4: Averaged system matrices of the stand-alone SSBI system in the $d q$-frame of

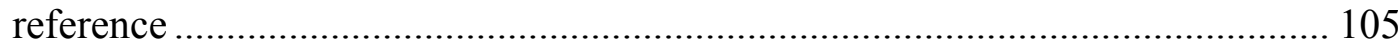

Table 5.5: System matrices for the switching states of the stand-alone SSBI in the $d q$ -

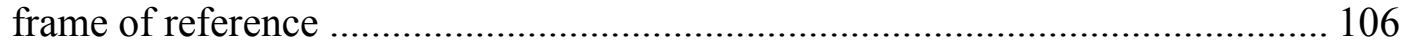

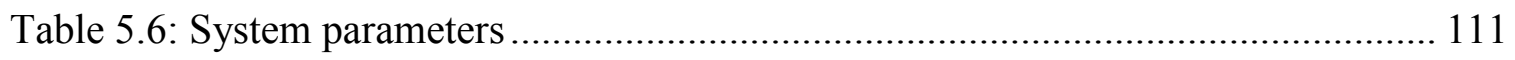

Table 5.7: Mean Absolute error of the state-space-averaged model for different switching and sampling frequencies 122 


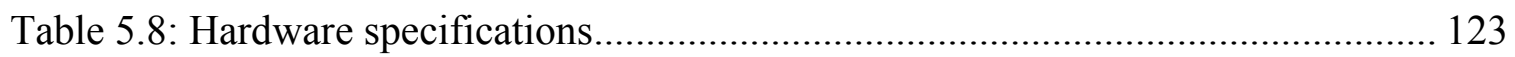

Table 6.1: The possible states of the grid-connected SSBI system .............................. 130

Table 6.2: Averaged system matrices of the grid-connected SSBI system for all sectors

Table 6.3: Averaged system matrices of the grid-connected SSBI system in the $d q$-frame of reference. 139

Table 6.4: System matrices for the switching states of the grid-connected SSBI in the $d q$ -

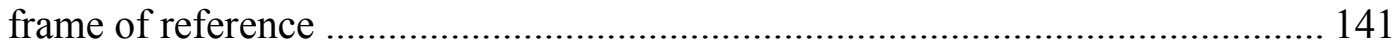

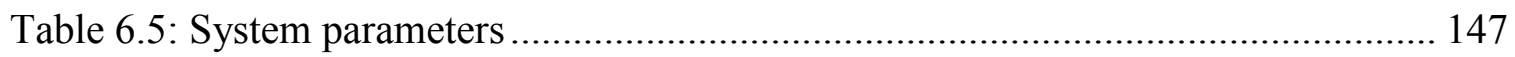

Table 6.6: Mean Absolute error of the state-space-averaged model for different sampling

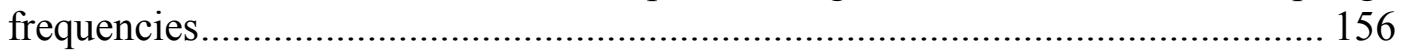

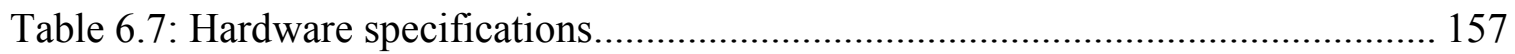




\section{LIST OF FIGURES}

FIGURE

PAGE

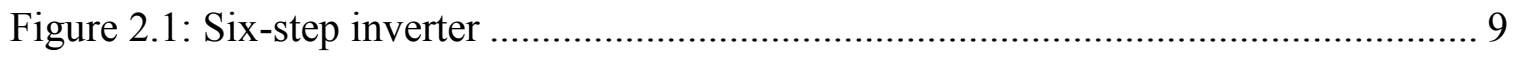

Figure 2.2: Gate signals and line-to-line voltages of six-step inverter .......................... 9

Figure 2.3: Sinusoidal PWM: control and carrier wavefroms, gate signals, and line-to-line voltages 12

Figure 2.4: Variations of the fundamental component of the output voltage versus the modulation index

Figure 2.5: Waveform of a selective harmonic elimination with two degrees of freedom 15

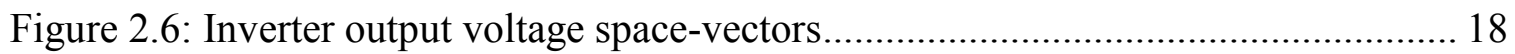

Figure 2.7: Three-phase three-level diode-clamped inverter ..................................... 20

Figure 2.8: Line-to-line voltage waveform for a three-level diode-clamped inverter ...... 21

Figure 2.9: Three-phase three-level flying-capacitor inverter .................................. 21

Figure 2.10: Three-phase three-level flying-capacitor inverter ................................... 22

Figure 2.11: Basic single-stage power conversion system, consisting of series connected dc-sources 25

Figure 2.12: Transformer-based two-stage conversion system 26

Figure 2.13: Transformer-less two-stage conversion system, consisting of a dc-dc boost converter cascaded with a PWM buck VSI

Figure 2.14: Single-stage Z-source inverter. 29

Figure 2.15: Single-stage current source boost inverter .

Figure 3.1: Inductor volt-second balance in a dc-dc boost converter, a) Schematic diagram of the boost converter, b) voltage and current profiles of the inductor under steady-state conditions. 
Figure 3.2: Steady-state modeling of a three-phase sinusoidal PWM inverter by averaging, a) schematic diagram of the inverter, b) PWM signals and the voltage between phase $\mathrm{A}$ and the neutral

Figure 3.3: Concept of the state-space averaging method, applied to a dc-dc boost converter, operating in continuous conduction mode (CCM) 40

Figure 3.4: An equivalent linear circuit for the dc-dc boost converter in CCM 43

Figure 3.5: Three-terminal equivalent circuit for a switch-diode combination used in dcdc boost converters, a) switch-diode combination, b) the equivalent three-terminal circuit

Figure 3.6: The third possible operating state, i.e. inductor discontinuous current mode (DICM), of a dc-dc boost converter under DCM. The other states have been already presented in Figure 3.3 .................................................................. 46

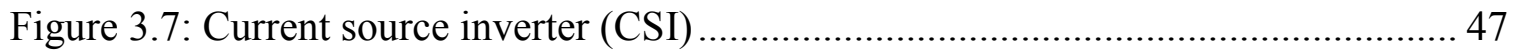

Figure 3.8: The equivalent linear circuit of the CSI of Figure 3.7 ............................. 48

Figure 3.9: The time-varying-transformer-based equivalent circuit of the CSI of Figure 2.13.

Figure 3.10: The concept of reduced order model, a) three-phase boost rectifier, b) active topology over the $60^{\circ}$ line period interval, in which phase $a$ has the highest negative voltage

Figure 3.11: $d q$-transformation for three-phase circuit quantities portrayed by trigonometric relationships 53

Figure 4.1: Complete power circuit schematic diagram of the CSI-based boost inverter 57

Figure 4.2: Line-to-line voltage phasors and the associated sectors 59

Figure 4.3: Sector (I), calculation of the discharging duty ratios, $d 1$ and $d 2$, as well as the charging duty ratio, $d c$

Figure 4.4: The charging (C1-C3) and discharging (D1-D6) states of operation for the SSBI

Figure 4.5: The voltage and current waveform of the dc-link inductor over one switching cycle in Sector (I). 64

Figure 4.6: The experimental prototype. 69 
Figure 4.7: Waveforms of the SSBI for the linear RL load, a) simulation, b) experimental

Figure 4.8: FFT Spectrum of the load terminal voltage and current for the linear RL load 71

Figure 4.9: Waveforms of the SSBI for the nonlinear rectifier load, a) simulation, b)

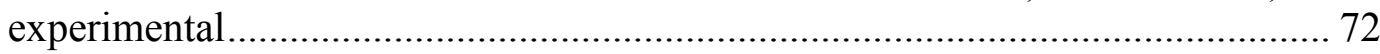

Figure 4.10: FFT Spectrum of the load terminal voltage and current for the nonlinear

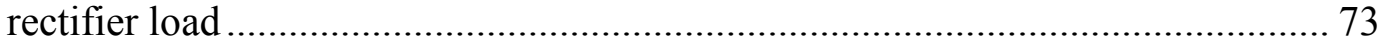

Figure 4.11: Short circuit fault on the dc-ac conversion systems ................................. 74

Figure 4.12: Performance of the inverters under an LG short circuit fault, a) SSBI, b)

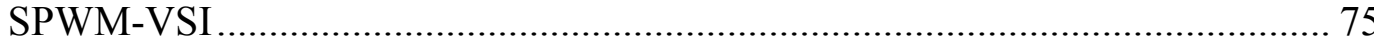

Figure 4.13: Performance of the inverters under an LL short circuit fault, a) SSBI, b)

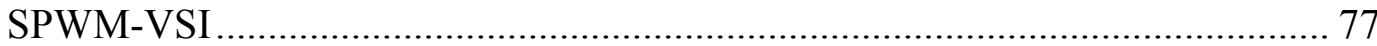

Figure 4.14: Performance of the inverters under an LLL short circuit fault, a) SSBI, b)

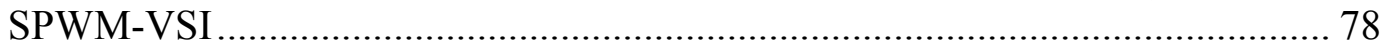

Figure 4.15: The complete power circuit schematic diagram of the CSI-based singlephase boost inverter

Figure 4.16: The switching pattern, one switch is modulated over each positive or negative half cycle in the single-phase SSBI ......................................... 80

Figure 4.17: Switch positions, positive (Mode I) and negative (Mode II) half cycles ..... 80

Figure 4.18: Performance of the single-stage boost inverter at the MPP of BP3230T, a)

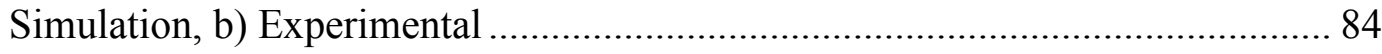

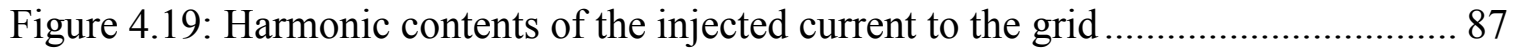

Figure 4.20: Variations of the dc-source power and the injected power to the grid versus the modulation index 90

Figure 4.21: Variations of the efficiency and the THD of the single-stage boost inverter versus its injected power to the grid

Figure 4.22: Variations of the overall efficiency and the THD of the single-stage boost inverter versus the modulation index 
Figure 5.1: Stand-alone three-phase single-stage boost inverter system

Figure 5.2: Locations of the stand-alone SSBI eigenvalues and their sensitivities versus

$R d c$ when it varies between 0.001 and $1 \Omega$ 112

Figure 5.3: Locations of the stand-alone SSBI eigenvalues and their sensitivities versus $L d c$ when it varies between 5 and $20 \mathrm{mH}$.....

Figure 5.4: Locations of the stand-alone SSBI eigenvalues and their sensitivities versus Cac when it varies between 5 and $20 \mu F$

Figure 5.5: Locations of the stand-alone SSBI eigenvalues and their sensitivities versus

$R L$ when it varies between 100 and $500 \Omega$ 115

Figure 5.6: Locations of the stand-alone SSBI eigenvalues and their sensitivities versus $L L$ when it varies between 0.5 and $5 \mathrm{mH}$ 116

Figure 5.7: Locations of the stand-alone SSBI eigenvalues and their sensitivities versus $M$ when it varies between 0.2 and 0.5 117

Figure 5.8: State variables of the simulation and state-space-averaged model for $f s=$ $2.88 \mathrm{kHz}$ and $f s a m=86 \mathrm{kHz}$. 119

Figure 5.9: State variables of the simulation and state-space-averaged model for $f s=$ $2.8 \mathrm{kHz}$ and $\mathrm{fsam}=280 \mathrm{kHz}$. 121

Figure 5.10: Start-up operation of the lab-scaled circuit and the response of the statespace-averaged model for $f s=2.88 \mathrm{kHz}$ and $f s a m=86 \mathrm{kHz}$ 124

Figure 5.11: $d q$ state variables of the lab-scaled circuit and the state-space-averaged model during the start-up for $f s=2.88 \mathrm{kHz}$ and $f \mathrm{sam}=86 \mathrm{kHz}$

Figure 5.12: Step responses of the hardware and the state-space-averaged model, to a 3\% increase in $m$, for $f s=2.88 \mathrm{kHz}$ and $f s a m=86 \mathrm{kHz}$

Figure 6.1: Grid-connected three-phase single-stage boost inverter system 129

Figure 6.2: Locations of the grid-connected SSBI eigenvalues and their sensitivities versus $R d c$ when it varies between 0.01 and $1 \Omega$. 148

Figure 6.3: Locations of the grid-connected SSBI eigenvalues and their sensitivities versus $L d c$ when it varies between 10 and $20 \mathrm{mH}$. 
Figure 6.4: Locations of the grid-connected SSBI eigenvalues and their sensitivities versus $C a c$ when it varies between 5 and $30 \mu F$

Figure 6.5: Locations of the grid-connected SSBI eigenvalues and their sensitivities versus $R g$ when it varies between 0.01 and $5 \Omega$

Figure 6.6: Locations of the stand-alone SSBI eigenvalues and their sensitivities versus $L g$ when it varies between 15 and $50 \mathrm{mH}$.

Figure 6.7: Locations of the grid-connected SSBI eigenvalues and their sensitivities versus $M$ when it varies between 0.2 and 0.5

Figure 6.8: State variables of the simulation and state-space-averaged model for $f s=$ $2.88 \mathrm{kHz}$ and $f \mathrm{sam}=2000 \mathrm{kHz}$. 155

Figure 6.9: Start-up operation of the lab-scaled circuit and the response of the statespace-averaged model (with an ideal dc-source) for $f s=2.88 \mathrm{kHz}$ and $f s a m=$ $81 \mathrm{kHz}$ 158

Figure 6.10: $d q$ state variables of the lab-scaled circuit and the state-space-averaged model (with an ideal dc-source) during the start-up for $f s=2.88 \mathrm{kHz}$ and fsam $=81 \mathrm{kHz}$

Figure 6.11: Step responses of the hardware and the state-space-averaged model, to a 3\% increase in $m$, for $f s=2.88 \mathrm{kHz}$ and $f s a m=81 \mathrm{kHz}$ 161

Figure 6.12: Step responses of the hardware and the state-space-averaged model, to a $-3^{\circ}$ decrease in $\phi$, for $f s=2.88 \mathrm{kHz}$ and $f s a m=81 \mathrm{kHz}$ 162

Figure 6.13: Start-up operation of the lab-scaled circuit and the response of the statespace-averaged model, considering the dc-source abnormality, for $f s=2.88 \mathrm{kHz}$ and $f$ sam $=81 \mathrm{kHz}$ 163

Figure 6.14: $d q$ state variables of the lab-scaled circuit and the state-space-averaged model, considering the dc-source abnormality, during the start-up for $f s=$ $2.88 \mathrm{kHz}$ and $f \mathrm{sam}=81 \mathrm{kHz}$ 


\section{CHAPTER 1}

\section{INTRODUCTION}

Renewable or sustainable energy (SE) technologies such as photovoltaic (PV) panels, wind generators (WG), fuels cells (FC), and energy storage (ES) devices, have attracted widespread attention during the last decade. This is because their generated power is environmentally friendly, and the sources are not subject to the instability of price and availability that are common to the conventional energy sources like oil and gas. In this way, advancements in semiconductor technology have led to a large utilization of SE sources and an increasing penetration of distributed generation (DG) units into existing energy infrastructures. In these systems, typically a low dc voltage, which is provided from a SE source or ES device, must be boosted and converted to an ac voltage with a fixed frequency and amplitude. Power electronic converters have been widely used for this purpose.

Several power electronic circuit topologies, for the purpose of converting the output of low dc voltage sources to the line ac voltage, have been proposed in literature [1-22]. A conventional power conversion system typically involves two stages of conversion, in which, a dc-dc converter is first used to isolate and/or boost the variable low-voltage input to a fixed dc output, and then a dc-ac converter (inverter) is employed to produce a sinusoidal output for either a stand-alone or a grid-connected conversion system. In order to realize the economic and environmental benefits of the low voltage dc-sources, like sustainable energy sources and ES systems, a significant amount of effort 
is required to develop novel power electronic circuit topologies as the interfaces between the low voltage de generation units and local loads and/or the grid.

The current source inverter (CSI) topology, along with the associated switching patterns and control schemes, is capable of converting the low de voltage to the line ac in only one stage. A switching pattern for the three-phase single-stage current source boost inverter has been recently proposed in [4]. Besides the simplicity of the proposed switching pattern, elimination of the electrolytic capacitors is one of the greatest advantages of this inverter, which can lead to a significant improvement in its reliability and lifetime. This feature is particularly interesting for photovoltaic energy conversion systems, in which the average lifetime of PV panels is about 20 years [6].

\subsection{Motivation}

In DG systems, inverters play key roles in connecting SE sources to the local loads and the ac grid. Although there has been a rapid expansion in the use of SE sources in recent years, there has been a lack of fundamental research on the design of inverters that are specialized for use in DG systems. Hence, there is a need to reconsider the design of energy conversion systems based on the specifications, limitations, and requirements of different DG technologies. Recent advances in power electronics have led to proposing new topologies as well as switching patterns and control algorithms for single-stage electric power conversion systems [3-5, 8-22], which are appropriate for SE sources and ES devices. The new topologies, including the associated control schemes, can convert a low dc voltage to the line ac voltage in only one stage. In comparison with the conventional two- or multi-stage conversion systems, the single-stage conversion results 
in reduction in size and cost of the system as well as an increase in its efficiency and reliability. However, like any emerging technology, the single-stage boost inverter (SSBI) can have as many disadvantages as advantages. The potential challenges, associated with the SSBI, must be addressed, investigated, and solved, in order to commercialize the technology, and make it a viable competitor to the existing technologies. Interconnection issues between the solid-state power converters and local networks (like a utility grid or local loads), stability and dynamics, protection, management, active and reactive power control, in either steady-state or transient conditions, and performance under abnormal operation, can be considered as some of the major technical concerns in design and implementation of the SSBI systems.

Steady-state and dynamic models are of the most essential requirements for performance analysis and control design of any engineering system. Thus, in order to have a satisfactory operation, it is necessary to derive the steady-state and dynamic models for the SSBI system. The developed models can be linearized around any operating point in order to derive the state-space representation of the system, as well as the transfer functions associated with different inputs and outputs of the system. These models and transfer functions can be used for many studies like frequency response analysis, stability, voltage regulation, seamless transition between stand-alone and gridconnected operational modes, filter design, and performing control studies of the system such as controllability and observability, nonlinear and intelligent control, and optimal and adaptive control. However, it should be noted that, because of their switching behavior and nonlinear elements, the analysis of power electronic converters is generally considered as a complicated task [23]. 


\subsection{Problem Statement and Research Objectives}

This study aims to derive the state-space-averaged model of the single-stage boost inverter, proposed in [4]. Then, the model will be used for sensitivity analysis of the SSBI system.

Since the SSBI can operate in two different modes, i.e. stand-alone and gridconnected, and each mode is associated with specific circuitry, the problem has to be split into two sub-problems. This results in developing two different large-signal state-spaceaveraged models, one for the stand-alone and the other one for the grid-connected SSBI systems. However, the obtained state-space-averaged models are nonlinear with respect to the control parameters, modulation index and angle. In order to consider these parameters as inputs to the system, small-signal analyses have to be performed. This can be done through perturbation and linearization techniques.

The small-signal models may be used for calculating the eigenvalues of the SSBI system. The placement of the eigenvalues on the $s$-plane is a measure of the stability of the SSBI system. An eigenvalue sensitivity analysis should be performed in order to investigate the stability and dynamic behavior of the system over a typical range of operation.

A variety of operating conditions such as short circuit faults, grid interruption, islanded operation, component outage, non-ideality of the dc-source, loss of load, pulse loads, harmonic loads, unbalanced operation, and the switching of large loads can all be considered as abnormal conditions in a single-stage boost-inverter system. However, in this study we are mainly interested in addressing the non-ideality of the dc-source. 
The validity of the findings of these studies has to be verified through simulation and experiments. Therefore, the stand-alone and grid-connected SSBI systems, as well as their state-space-averaged models must to be simulated, and an experimental hardware set-up, including a laboratory-scaled prototype SSBI, has to be built and tested. The obtained models are not valid, unless the outputs of the model follow those of the simulations and experiments.

The state-space-averaged models are usually developed for ideal systems, in which all non-idealities like voltage drops and delays of the semiconductor devices (switches and diodes) as well as non-linearity of the circuit components are neglected. However, in practice, these devices do not behave perfectly. Since, the non-idealities affect the accuracy of the derived state-space-averaged models, it would be great if the averaged models could be modified in a way that the effects of the aforementioned nonidealities could be included, and the models become practically applicable.

Accordingly, the objectives of this $\mathrm{PhD}$ dissertation can be summarized as follows:

i. Presenting and discussing the concept, topology, switching pattern, control strategy, and performance of the recently proposed [4] SSBI,

ii. Developing the large-signal state-space-averaged models, for the stand-alone and grid-connected, SSBI systems,

iii. Applying perturbation and linearization method in order to derive the small-signal models of the SSBI systems, with the control parameters, modulation index and angle, as inputs,

iv. Verifying the validity of the obtained models through simulation and experiments, 
v. Performing sensitivity analysis by inspecting the loci of the system eigenvalues on the s-plane,

vi. Investigating the performance of the SSBI systems under the abnormal condition of variations in the dc-source voltage.

\subsection{Dissertation Roadmap}

The rest of this dissertation is organized as follows: Chapters 2 presents a comprehensive review of the existing dc-ac power conversion systems, used for sustainable and renewable-based distributed generation applications.

An extensive literature survey of related studies on the steady-state and dynamic modeling of power electronic converters is presented in Chapter 3, in which the previous and current achievements are categorized in relation to the work proposed in this dissertation.

The recently proposed single-stage boost inverter [4] is elaborated upon in Chapter 4. The circuit topology, switching pattern, control strategy, and steady-state performance of the single- and three-phase SSBIs are explained and discussed in details and the concept is proved through simulation and experiments.

The large- and small-signal state-space-averaged models of the stand-alone SSBI, in the synchronous $d q$-frame of reference, are developed in Chapter 5. The dynamic behavior of system is investigated by analyzing their responses to different inputs. This is achieved by applying step changes in the dc-source voltage and the modulation index. The validity of the obtained models is verified through simulation and experiments. Then, 
the loci of the system's eigenvalues are plotted and the sensitivity of the stability, speed, and oscillation of the system with respect to its different parameters are discussed.

Chapter 6 includes the same tasks as performed in Chapter 5, but for the gridconnected SSBI inverter. The differences arise from the presence of the grid, which adds to the complexity of the models. Finally, the dynamics of the system, as well as the response of the models, under abnormal operation of the dc-source is investigated.

Chapter 7 provides the conclusion of the dissertation and identifies future research subjects.

Overviews of the averaging theory as well as perturbation and linearization technique, which are key concepts of this work, are presented in the Appendices. 


\section{CHAPTER 2}

\section{DC-AC CONVERSION IN DISTRIBUTED GENERATION SYSTEMS}

This chapter provides a review of the history and contribution of existing literature that relates to dc-ac power conversion systems. The focus of the work is on sustainable- and renewable-based distributed generation systems, in which the converters have to produce high voltages from low voltage dc inputs.

This chapter contains five sections. The six-step inverter is introduced in Section 1. Four major types of pulse-width-modulated inverters are presented in Section 2. Section 3 reviews three common configurations of multilevel inverters. The background work, related to existing boost-inverting power conversion systems for distributed generation applications are reviewed in Section 4, and Section 5 is the summary of the chapter.

\subsection{Six-Step Inverter}

The six-step inverter, with an H-bridge topology, is the simplest type of dc-ac conversion system. The three-phase H-bridge converter topology, which serves as the basis for nearly all three-phase inverters, is presented in Figure 2.1. Metal-oxide fieldeffect transistors (MOSFETs) have been used as switches in this figure. However, different types of semiconductor controllable switches, like bipolar junction transistors (BJTs), insulated-gate bipolar junction transistors (IGBTs), and metal-on-silicon controlled thyristors (MCTs), can be used in this inverter [28]. 


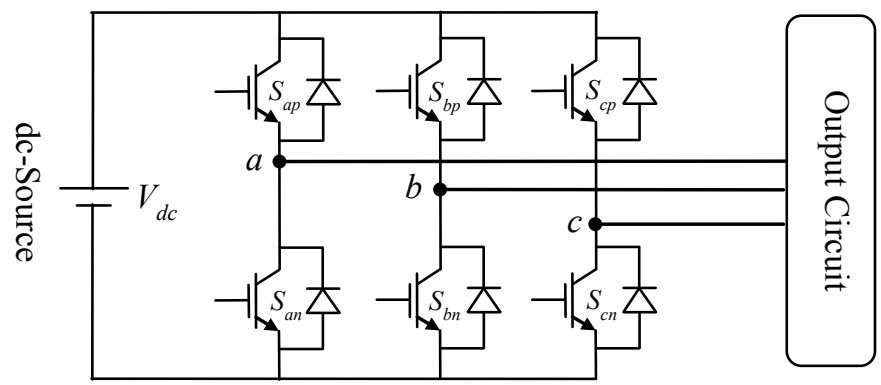

Figure 2.1: Six-step inverter

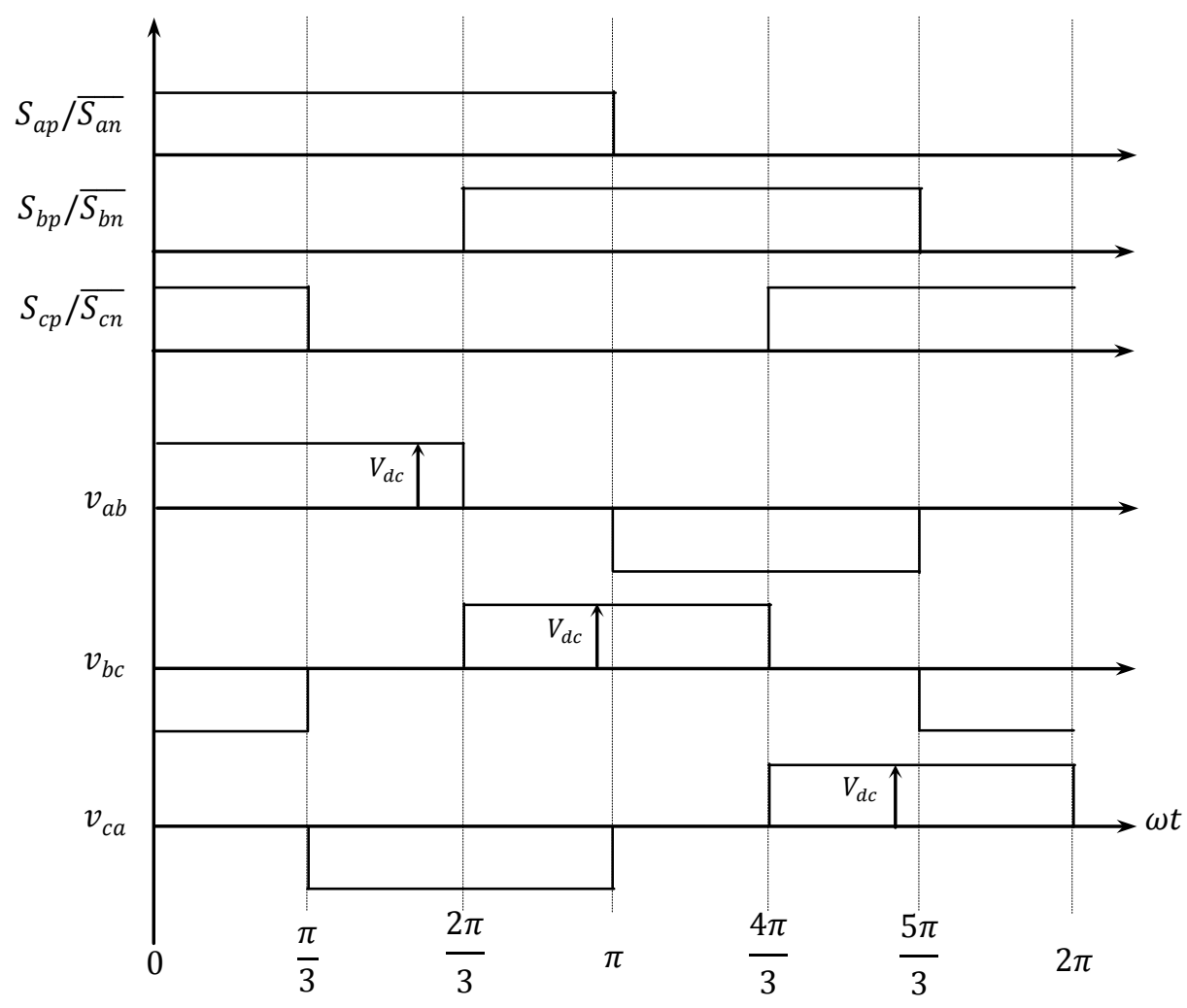

Figure 2.2: Gate signals and line-to-line voltages of six-step inverter 
The gate signals that are driving the switches of a six-step inverter, and its line-toline output voltages are shown in Figure 2.2, where the hat sign $\left(^{-}\right)$denotes the logical complement. As can be seen in this figure, each switch conducts for 180 electrical degrees per each switching cycle. Also, using this figure, one can calculate the line-toline rms voltage of the six-step inverter:

$V_{L L}=\sqrt{\frac{1}{2 \pi} V_{d c}^{2}\left(\frac{4 \pi}{3}\right)}=\sqrt{\frac{2}{3}} V_{d c}$

This equation indicates that the output voltage of the six-step inverter is only a function of the applied dc voltage; therefore, the output voltage cannot be regulated unless the applied dc voltage is adjusted. High total harmonic distortion (THD), especially at loworder harmonics of $\{5,7,11,13,17,19, \ldots\}$, is another major disadvantage of the sixstep inverter. This is due to the square waveform of the generated voltage.

\subsection{Pulse-Width-Modulated Inverter}

Similar to the six-step inverter, the output line-to-line voltage of this type of inverter can be at three voltage levels, i.e. $-V_{d c}, 0$, and $V_{d c}$. However, a pulse-widthmodulation (PWM) strategy allows the rms voltage (and amplitude of the fundamental component) to be readily controlled. There are various schemes to pulse-width modulate the inverter switches. Four major types of these schemes, i.e. sinusoidal PWM, thirdharmonic injection, selective harmonic elimination, and space-vector modulation, will be discussed in the following subsections. 


\subsubsection{Sinusoidal Pulse-Width-Modulation (SPWM)}

Sinusoidal pulse-with-modulation is based on generating a sequence of voltage pulses at a certain frequency and sinusoidal modulated pulse-widths. This can be achieved through comparing three sine-wave control voltages, $v_{c, a}, v_{c, b}$, and $v_{c, c}$, with a triangular waveform, $v_{t r i}$, which is called the carrier. The ratio between the amplitude of the control voltages, $V_{c}$, and peak of the triangular voltage, $\widehat{V}_{t r i}$, is defined as the modulation index, $m=V_{c} / \widehat{V}_{t r i}$. The frequency of the triangular waveform, which is usually kept constant, establishes the inverter switching frequency, and the frequency of the control voltages determines the output voltage frequency. Because of its simplicity and ease of implementation, the SPWM is widely used in industrial applications [84]. The concept of PWM, applied to a three-phase H-bridge converter topology is shown in Figure 2.3.

By changing the modulation index, $m$, the widths of pulses vary, which results in variations in the amplitude of the output voltage. Another advantage of the SPWM over six-step switching pattern is its higher quality voltage waveform. The harmonics in the output voltage appears as sidebands, centered around the switching frequency, $f_{s}$, and its multiples. If the ratio between the switching frequency and the desired output frequency, $f_{1}$, is defined as the modulation frequency ratio, i.e. $m_{f}=f_{s} / f_{1}$, the frequency spectrum of the output voltage of a SWPM inverter contains harmonics orders around $m_{f}, 2 m_{f}$, $3 m_{f}$, and so forth [25]. It is also worth mentioning that for a frequency modulation ratio $m_{f} \geq 9$ (which is always true except for very high-power applications), the harmonic amplitudes are almost independent of $m_{f}$. Moreover, odd integer modulation frequency 
ratios are recommended for avoiding even-order harmonics, and in particular a small dc component, at the output voltage [85].

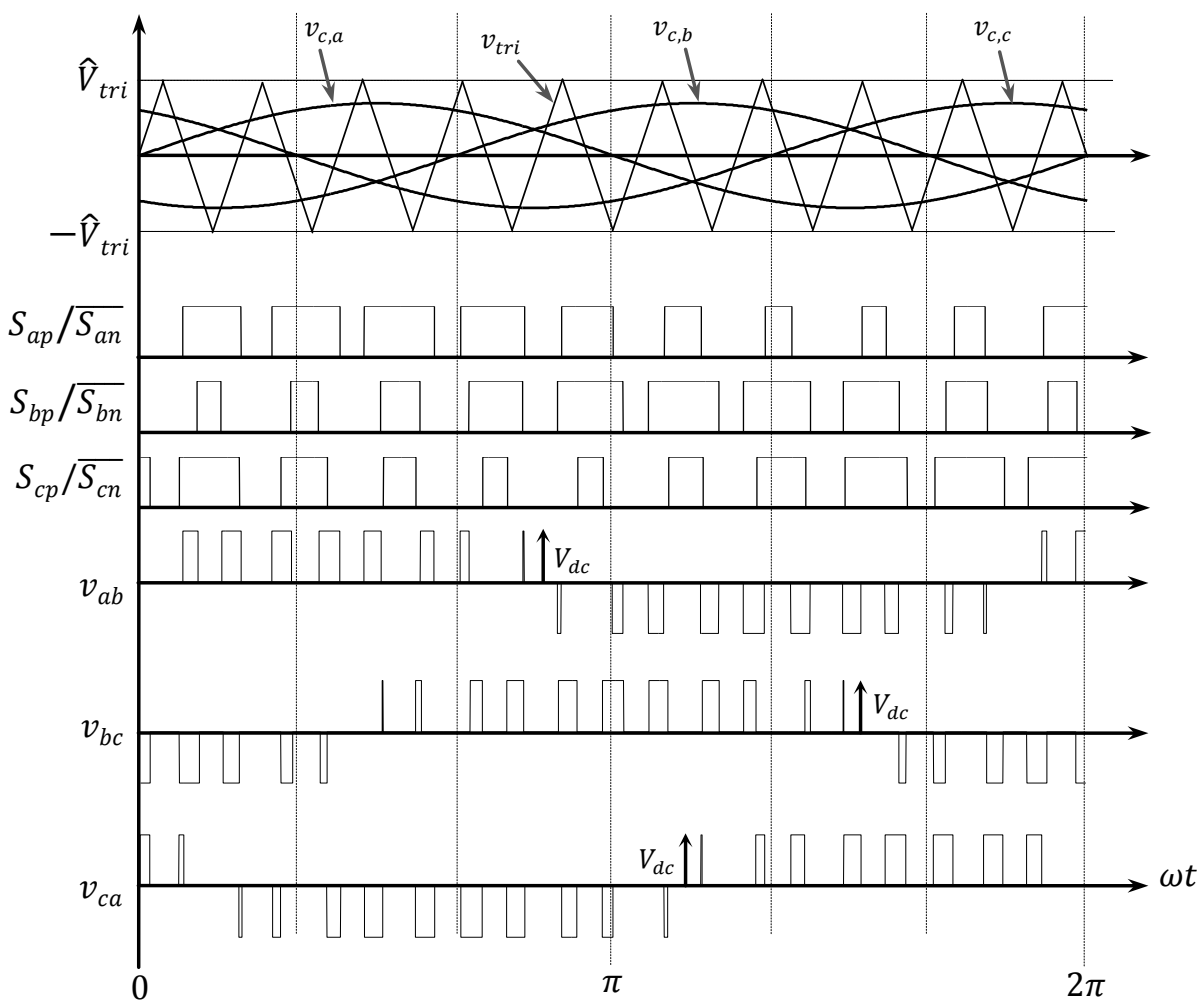

Figure 2.3: Sinusoidal PWM: control and carrier wavefroms, gate signals, and line-to-line voltages

Besides the harmonic components, an inappropriate choice of $m_{f}$ may cause subharmonics in the output voltage of the SWPM inverters. The sub-harmonics may occur when $m_{f} \leq 21$ and it is not an integer number. Thus, in order to avoid sub-harmonics the modulation frequency ratio should be an integer number, this is so-called synchronous $P W M$. On the other hand, asynchronous $P W M$, in which the frequency modulation ratio is not an integer number, can be applied when the modulation frequency ratio is 
sufficiently large, i.e. $m_{f} \geq 21$ [25]. However, it should be kept in mind that, increasing $m_{f}$ results in higher switching frequencies, which increase the switching losses and decrease the efficiency.

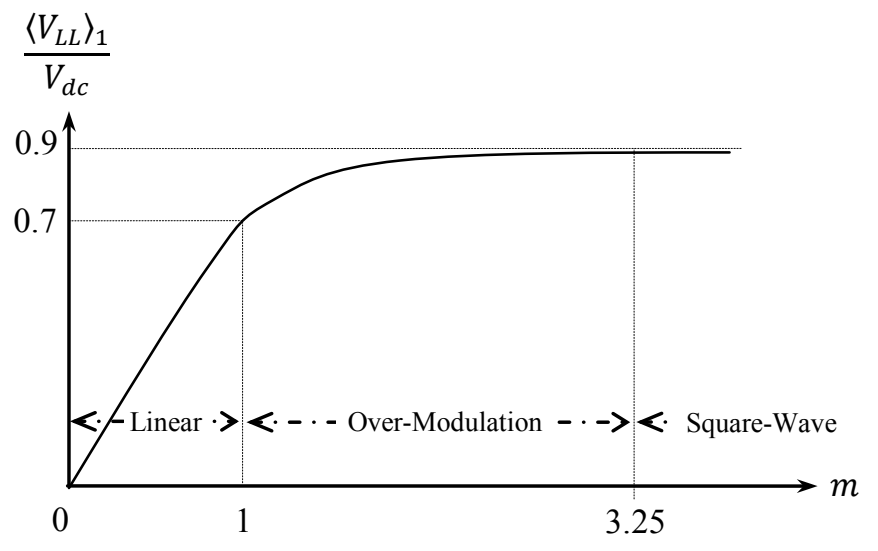

Figure 2.4: Variations of the fundamental component of the output voltage versus the modulation index

The waveforms of Figure 2.3 are a case with a modulation index $m \leq 1$ (linear range of $m$ ), in which the amplitude of the control signal is smaller than the peak of the carrier signal. However, it may happen that the modulation index exceeds 1. In this situation, which is called over-modulation, the control voltages may not intersect the triangular waveform for a period of time. In general, over-modulation causes the output voltage to contain many more harmonics in the sidebands as compared with the linear range. Furthermore, during over-modulation conditions, the amplitude of the fundamental component of the output voltage does not vary linearly with $m$, which is the case within the linear range of operation, i.e. $m \leq 1$. Moreover, it is observed that, as the modulation index increases beyond one, the output waveform turns more and more to square-wave, like that for a six-step inverter. The over-modulation effect on the fundamental 
component of line-to-line output voltage of the SPWM inverter, $\left\langle V_{L L}\right\rangle_{1}$, is shown in Figure 2.4.

\subsubsection{Third-Harmonic Injection}

As was mentioned in the previous section, the maximum value of high-quality output voltage can be achieved at the upper bound of the linear range of operation, $m=$ 1. This is one of the main limitations of SPWM. However, this limit can be increased by introducing a third harmonic to the control voltage. It has been shown in [28] that, with a proper choice of the fundamental and third-harmonic components for the control voltage, the inverter can output $15 \%$ more voltage without suffering from the consequences of over-modulation.

\subsubsection{Selective Harmonic Elimination}

Selective harmonic elimination is another PWM method that has low baseband distortion [87]. This method is particularly suitable for low switching frequency inverters, which are either high-power or have slow switching devices. This method is based on the idea of chopping the square-waveform of the six-step inverter at some certain angleintervals in order to eliminate some specific low-order harmonics from the output voltage waveform [86]. An example of selective harmonic elimination with two degrees of freedom, corresponding to one notch between 0 to $\pi / 2$, is demonstrated in Figure 2.5. In order to avoid even harmonics, it is assumed that the output waveform has quarter-wave and half-wave symmetry. The periodic waveform of line-to-line voltage, $v_{a b}$, can be written as follows: 
$v_{a b}(t)=\sum_{h=1}^{\infty} b_{h} \sin (h \omega t)$

where, the Fourier coefficient $b_{h}$ is defined as:

$b_{h}=\frac{2}{\pi} \int_{0}^{\pi} v_{a b}(t) \sin (h \omega t) d(\omega t)$

Because of the quarter-wave symmetry in Figure $2.5, b_{h}$ can be rewritten as:

$b_{h}=\frac{4}{\pi} \int_{0}^{\pi / 2} v_{a b}(t) \sin (h \omega t) d(\omega t)$

As a result:

$b_{h}=\frac{4}{\pi}\left[\int_{0}^{\alpha_{1}} v_{a b}(t) \sin (h \omega t) d(\omega t)+\int_{\alpha_{2}}^{\pi / 2} v_{a b}(t) \sin (h \omega t) d(\omega t)\right]$,

which can be simplified to:

$b_{h}=\frac{4 V_{d c}}{\pi h}\left[1-\cos \left(h \alpha_{1}\right)+\cos \left(h \alpha_{2}\right)\right]$

This equation can be solved for $\alpha_{1}$ and $\alpha_{2}$, to eliminate two selected harmonics. For instance, the following set of equation provides $\alpha_{1}$ and $\alpha_{2}$ for elimination of the $3^{\text {th }}$ and $5^{\text {th }}$ order harmonics from the line voltage waveform.

$\left\{\begin{array}{l}1-\cos \left(3 \alpha_{1}\right)+\cos \left(3 \alpha_{2}\right)=0 \\ 1-\cos \left(5 \alpha_{1}\right)+\cos \left(5 \alpha_{2}\right)=0\end{array} \Rightarrow\left\{\begin{array}{l}\alpha_{1}=17.83^{\circ} \\ \alpha_{2}=37.97^{\circ}\end{array}\right.\right.$

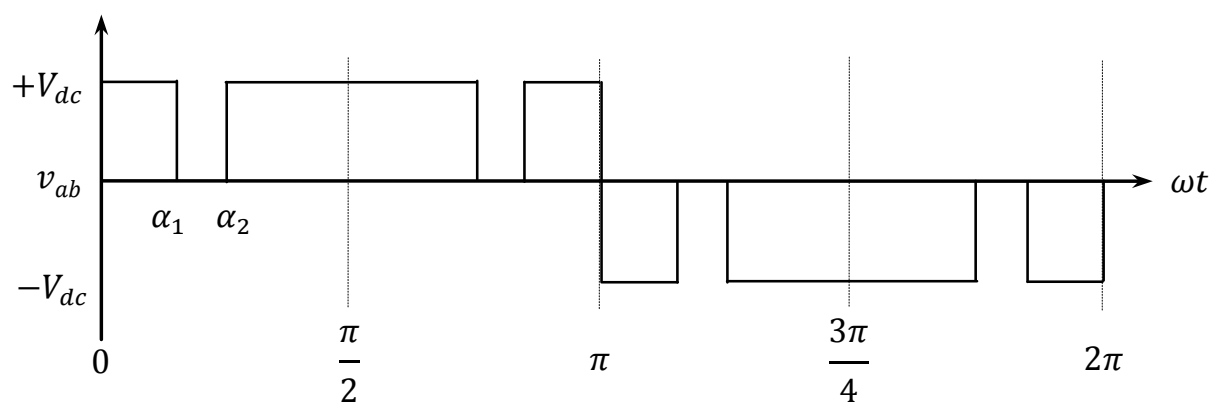

Figure 2.5: Waveform of a selective harmonic elimination with two degrees of freedom 
Accordingly, the methodology can be extended to eliminate more harmonic orders by introducing more notches between 0 to $\pi / 2$ (to increase the degrees of freedom of the equations).

\subsubsection{Space-Vector Modulation}

Space-vector pulse-with-modulation (SVPWM) is another technique of driving a voltage source three-phase H-bridge inverter, for generating voltage waveforms that are devoid of low-frequency harmonic content [90]. The approach is based on the spacevector representation of the output voltages, in which the three inverter voltages are represented by a voltage space-vector, $U$, defined as:

$U=\frac{2}{3}\left(V_{a b}+e^{j 2 \pi / 3} V_{b c}+e^{j 4 \pi / 3} V_{c a}\right)$

In total, the voltage source inverter (VSI) can operate in eight different states that are associated with eight switching positions. The switching table and the corresponding voltage space-vectors are presented in Table 2.1 and Figure 2.6, respectively. As can be seen in the figure, a reference voltage space-vector, $U^{r e f}$, can be developed by adding its two adjacent space-vectors. For instance, when the reference voltage space-vector is placed in Sector (I), it can be produced by operating the inverter is States 1 and 2, for duty ratios of $d_{1}$ and $d_{2}$, respectively. These duty ratios can be calculated by the wellknown law of sines, which is:

$\frac{d_{1}\left|U_{1}\right|}{\sin \left(\frac{\pi}{3}-\gamma\right)}=\frac{d_{2}\left|U_{2}\right|}{\sin (\gamma)}=\frac{\left|U^{r e f}\right|}{\sin \left(\frac{2 \pi}{3}\right)}$

Substituting the voltage space-vectors of Table 2.1 provides $d_{1}$ and $d_{2}$ as the following: 
$\left\{\begin{array}{l}d_{1}=\frac{\left|U^{r e f}\right|}{V_{d c}} \sin \left(\frac{\pi}{3}-\gamma\right) \\ d_{2}=\frac{\left|U^{r e f}\right|}{V_{d c}} \sin (\gamma)\end{array}\right.$

It should be noted that, these duty ratios are only valid if $d_{1}+d_{2} \leq 1$, i.e. when $U^{r e f}$ lies within the largest circle that can be circumscribed within the boundaries of the hexagon connecting the voltage space-vectors in Figure 2.6. The conditions that the reference voltage space-vector exceed the boundaries of the incircle, which is $\left|U^{r e f}\right|>V_{d c}$, is called over-modulation, which results in an increase in the harmonic content of the output voltage. On the other hand, once $d_{1}+d_{2}<1$, the inverter has to operate in the zero state (either State 0 or State 8 , or a combination of the both) for the rest of the switching cycle. This interval corresponds to a duty ratio of $d_{0}=1-d_{1}-d_{2}$.

It is also worth mentioning that to obtain the minimum switching frequency of each inverter leg, it is necessary to arrange the switching sequence in such a way that the transition from one state to the next is performed by switching only one inverter leg.

Table 2.1: Switching table and output voltages of a three-phase inverter

\begin{tabular}{ccccccccc}
\hline State & Sectors & $S_{a p} / \overline{S_{a n}}$ & $S_{b p} / \overline{S_{b n}}$ & $S_{c p} / \overline{S_{c n}}$ & $V_{a b}$ & $V_{b c}$ & $V_{c a}$ & Space-Vector, $U$ \\
\hline 0 & All & 0 & 0 & 0 & 0 & 0 & 0 & $U_{0}=0$ \\
\hline 1 & VI, I & 1 & 0 & 0 & $V_{d c}$ & 0 & $-V_{d c}$ & $U_{1}=2 / \sqrt{3} V_{d c} e^{j \pi / 6}$ \\
\hline 2 & I, II & 1 & 1 & 0 & 0 & $V_{d c}$ & $-V_{d c}$ & $U_{2}=2 / \sqrt{3} V_{d c} e^{j \pi / 2}$ \\
\hline 3 & II, III & 0 & 1 & 0 & $-V_{d c}$ & $V_{d c}$ & 0 & $U_{3}=2 / \sqrt{3} V_{d c} e^{j 5 \pi / 6}$ \\
\hline 4 & III, IV & 0 & 1 & 1 & $-V_{d c}$ & 0 & $V_{d c}$ & $U_{4}=2 / \sqrt{3} V_{d c} e^{j 7 \pi / 6}$ \\
\hline 5 & IV, V & 0 & 0 & 1 & 0 & $-V_{d c}$ & $V_{d c}$ & $U_{5}=2 / \sqrt{3} V_{d c} e^{j 3 \pi / 2}$ \\
\hline 6 & V, VI & 0 & 0 & 1 & $V_{d c}$ & $-V_{d c}$ & 0 & $U_{6}=2 / \sqrt{3} V_{d c} e^{j 11 \pi / 6}$ \\
\hline 7 & All & 1 & 1 & 1 & 0 & 0 & 0 & $U_{7}=0$ \\
\hline
\end{tabular}




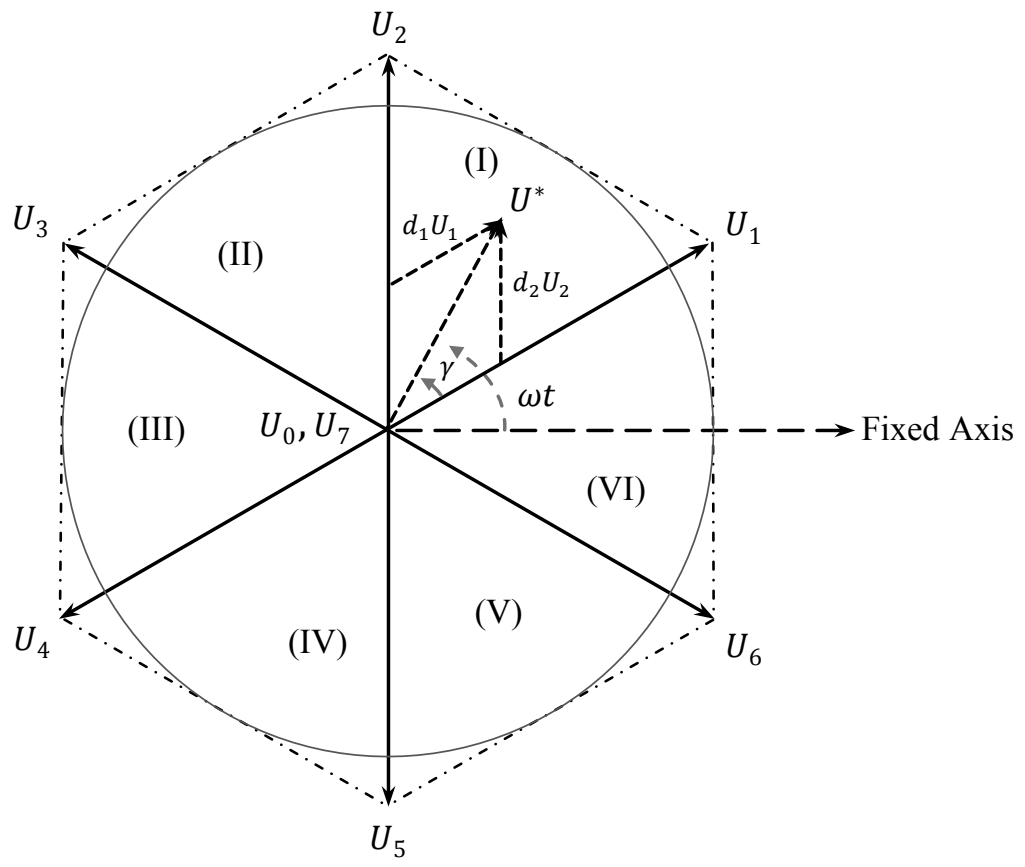

Figure 2.6: Inverter output voltage space-vectors

\subsection{Multilevel Inverter}

The multilevel inverter is another type of dc-ac converter which is more suitable for high-voltage and high-power applications. A major advantage of this inverter is a reduction in the harmonic content without increasing the switching frequency, or decreasing the inverter output power [91-93]. Besides the ability to provide a proper harmonic spectrum (making possible the use of smaller and less expensive filters), the multilevel inverter has a good dynamic response and relatively low voltage ratings for the switches. Moreover, multilevel inverters are a good tradeoff solution between performance and cost in high-voltage and high-power systems [11].

The output voltage waveform of the multilevel inverter is composed of a number of levels of voltage, typically obtained from capacitors or dc voltage sources. The so- 
called multilevel inverter starts at three levels, and as the number of levels increases the output THD decreases, and once the number of levels reaches infinity, the THD reaches zero. Multilevel inverters are categorized into three general topologies; diode-clamped multilevel inverter (DCMI), flying-capacitor multilevel inverter (FCMI), and cascaded multilevel inverter with separate dc sources.

\subsubsection{Diode-Clamped Multilevel Inverter (DCMI)}

This multilevel inverter has series capacitors at the dc-bus in order to divide the voltage into a set of voltage levels. In general, $M$-1 capacitors are used in an $M$-level diode-clamped inverter. It should be noted that the level of multilevel inverters are defined as the number of phase voltage levels, while the number of line-to-line voltage levels of a $M$-level inverter is $2 M-1$. A three-phase three-level diode-clamped inverter is shown in Figure 2.7. Each of the three phases share a common dc-bus, which has been subdivided by two capacitors into three levels. The voltage across each capacitor is $V_{d c} / 2$, and the voltage stress across each semiconductor switch is limited to $V_{d c} / 2$ through the clamping diodes. Table 2.2 lists the output phase voltage levels, with the negative dc rail voltage as a reference (point $n$ ), and the corresponding output line-to-line voltages are presented in Figure 2.8.

Table 2.2: Switching table of a three-level diode-clamped inverter

\begin{tabular}{ccccc}
\hline$v_{a n}$ & $S_{a p 1}$ & $S_{a p 2}$ & $S_{a n 1}$ & $S_{a n 2}$ \\
\hline$V_{d c}$ & 1 & 1 & 0 & 0 \\
\hline$V_{d c} / 2$ & 0 & 1 & 1 & 0 \\
\hline 0 & 0 & 0 & 1 & 1 \\
\hline
\end{tabular}


As can be seen in Figure 2.8, the output voltage waveform of a multilevel inverter has half-wave and quarter-wave symmetry. Thus, its Fourier series can be written as (2.2) and the Fourier coefficients can be obtained according to (2.4). In general, the Fourier coefficients of the line-to-line output voltage of an $M$-level inverter are obtained as the following:

$b_{h}= \begin{cases}\frac{4 V_{d c}}{M h \pi} \sum_{m=1}^{M-1} \cos \left(h \alpha_{m}\right) & ; \text { for odd } h s \\ 0 & ; \text { for even } h s\end{cases}$

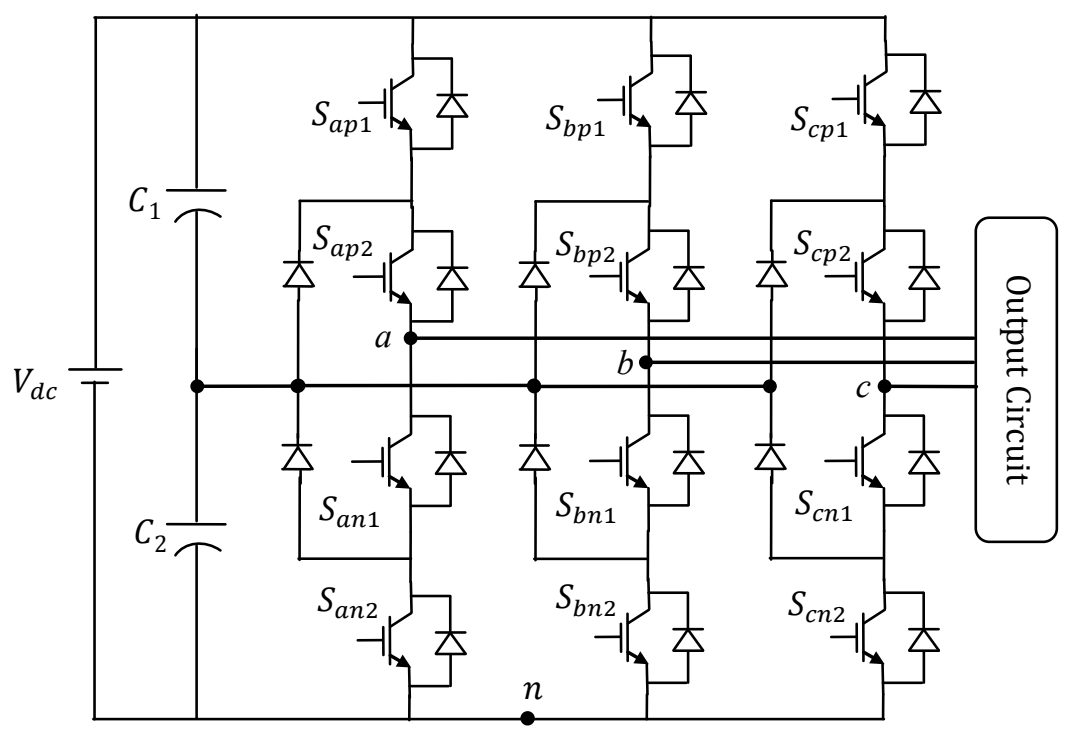

Figure 2.7: Three-phase three-level diode-clamped inverter 


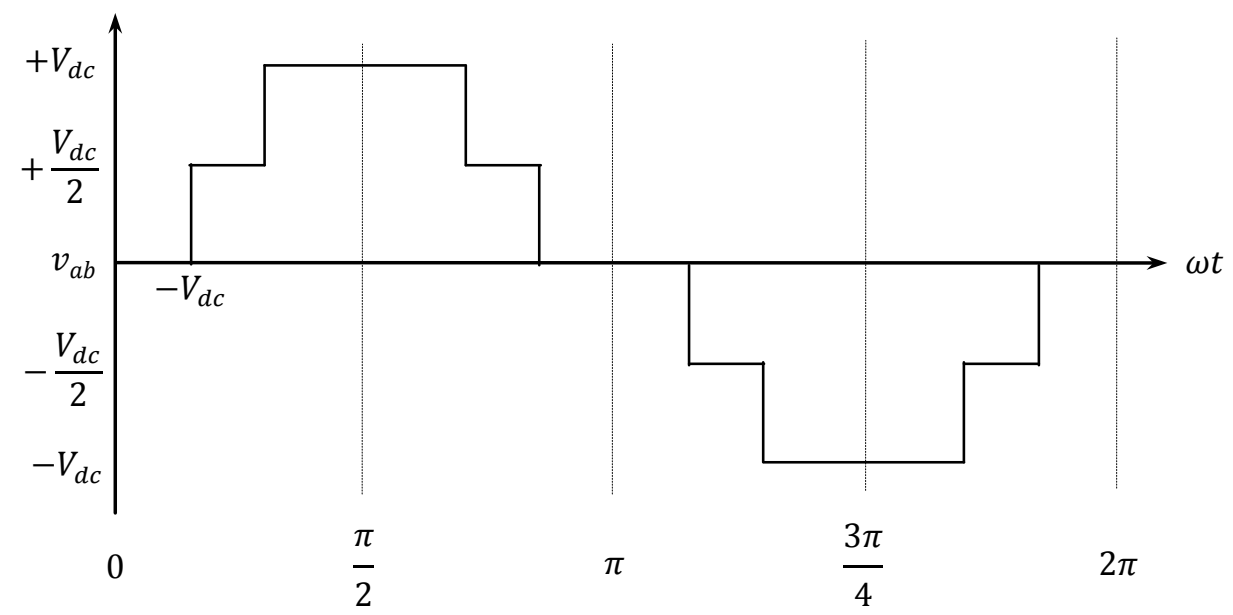

Figure 2.8: Line-to-line voltage waveform for a three-level diode-clamped inverter

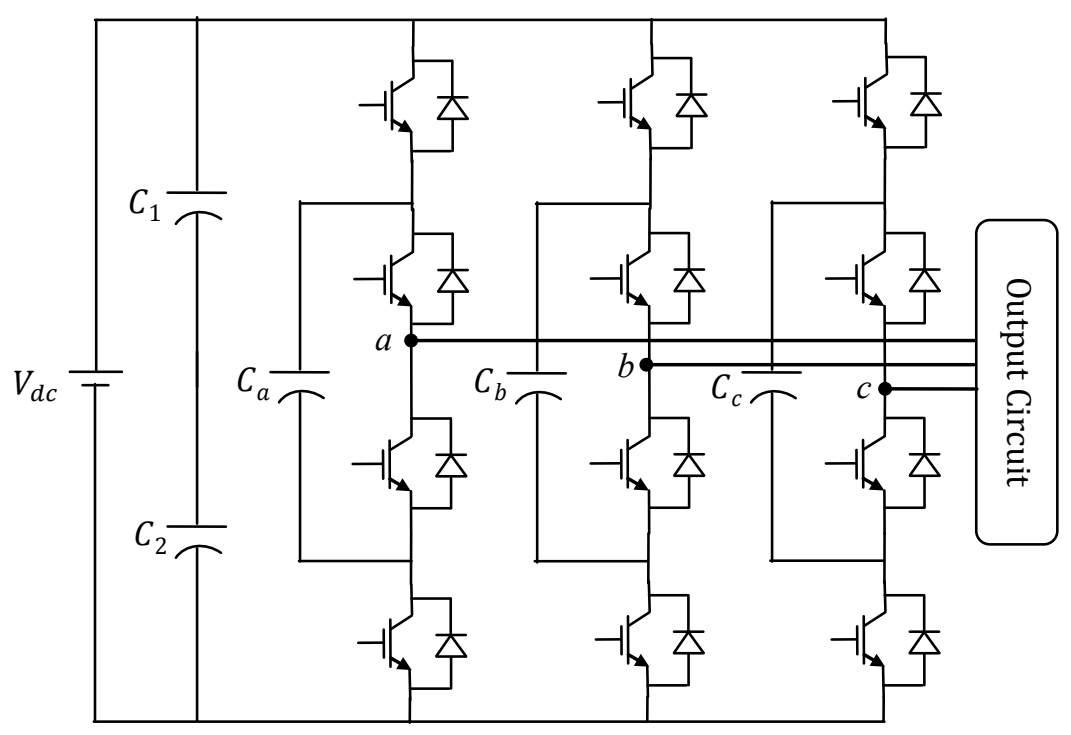

Figure 2.9: Three-phase three-level flying-capacitor inverter 


\subsubsection{Flying-Capacitor Multilevel Inverter (FCMI)}

The flying-capacitor multilevel inverter has a similar structure to the diodeclamped inverter except that is uses capacitors instead of clamping diodes. This inverter uses a ladder structure of dc-side capacitors, where each capacitor has a different voltage from the other capacitors [94-96]. The size of the voltage increment between two capacitors determines the size of the voltage levels in the output waveform. The circuit topology of three-level flying-capacitor inverter is shown in Figure 2.9.

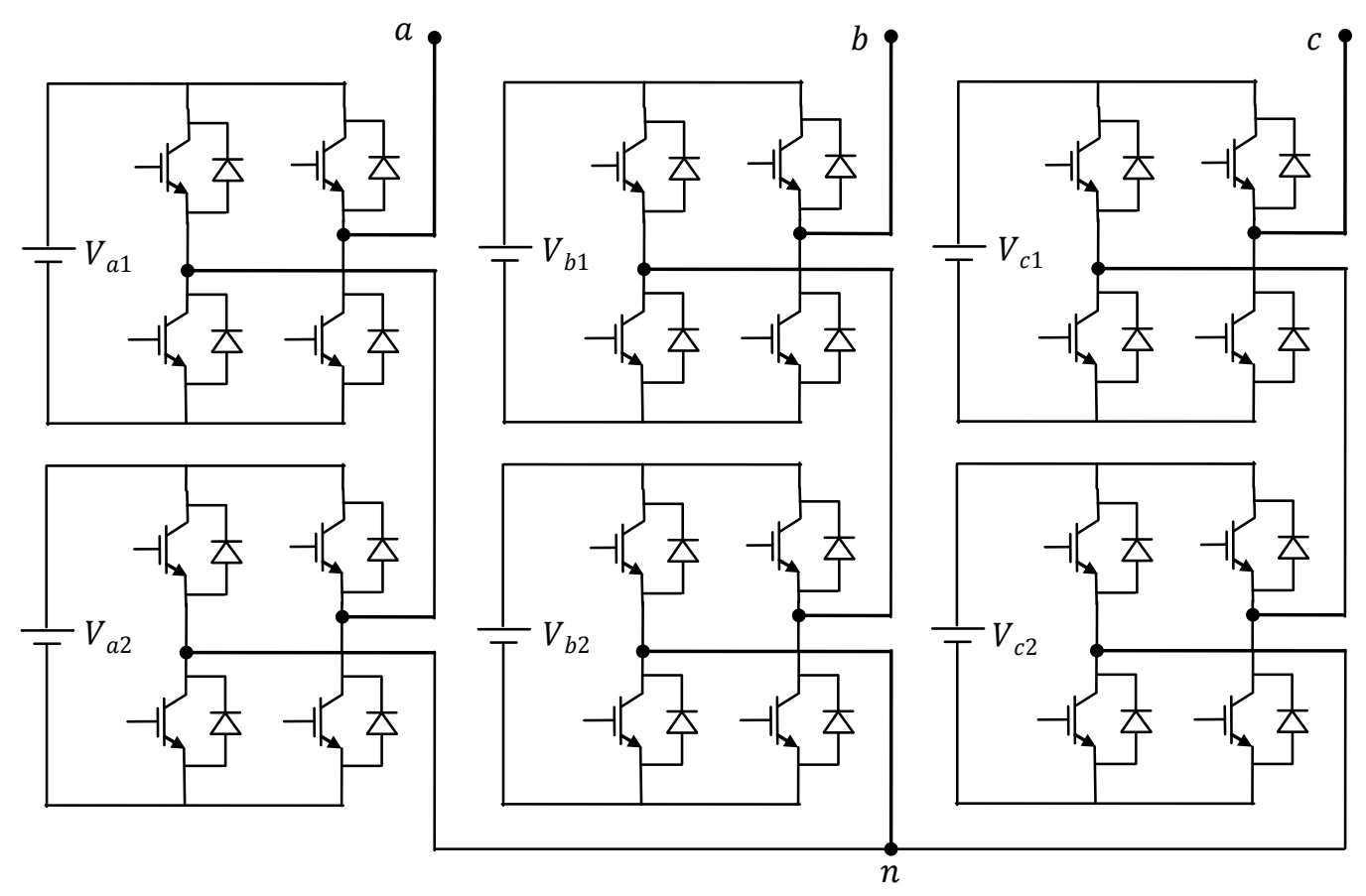

Figure 2.10: Three-phase three-level flying-capacitor inverter

In addition to the $(M-1)$ dc-bus capacitor, an $M$-level flying-capacitor inverter requires $(M-1) \times(M-2) / 2$ inner-loop (auxiliary) capacitors. It can be seen in Figure 2.9 that all phase legs share the same dc-link capacitors, $C_{1}$ and $C_{2}$. Also, the inner-loop balancing 
capacitors for each phase leg $\left(C_{a}, C_{b}\right.$ and $\left.C_{c}\right)$ are independent from those for the other legs. An advantage of the flying-capacitor multilevel inverter is that several switching combinations may be used to synthesize an output voltage. For instance, the switching table of Table 2.2 is one of the possible switching combinations for the three-level flying capacitor inverter of Figure 2.9.

\subsubsection{Cascaded-Inverters with Separate dc-Sources}

Cascaded-inverters with separate dc-sources are another prevalent structure for the multi-level inverter. Its functionality is similar to the other two types of multilevel inverters of the previous subsections, but it synthesizes a desired output voltage waveform from several independent dc-sources. Photovoltaic panels, fuel cells, and batteries, can be some of the possible choices. A three-phase, three-level configuration of the cascaded-inverter is illustrated in Figure 2.10. As can be seen, it is a combination of three Y-connected single-phase cascaded-inverters. Each inverter level can generate three different voltage outputs, e.g. $+V_{a 1}, 0$, and $+V_{a 1}$, by connecting the dc-source to the ac output by different combinations of the switches.

\subsubsection{A Comparison among the Multilevel Inverters}

A comparison between the three introduced types of multilevel inverters is presented in Table 2.3. It shows that all inverters need the same number of switches and main diodes in order to generate the same number of levels. The flying-capacitor and cascaded-inverters do not require clamping diodes, while the flying-capacitor inverter needs inner-loop balancing capacitors. Technically, the cascaded-inverter requires the 
least number of components, apart from the voltage sources. It has a modular circuit layout and its packaging is simple. The reason is that all levels have the same structure and no clamping diodes or balancing capacitors are needed. Moreover, by adding or removing the H-bridge cells, the number of output voltage levels can be easily adjusted.

Table 2.3: Comparison of power components, required per phase leg, among the multilevel inverters

\begin{tabular}{cccc}
\hline Inverter Configuration & Diode-Clamped & Flying-Capacitors & Cascaded-Inverters \\
\hline Switches & $2(M-1)$ & $2(M-1)$ & $2(M-1)$ \\
\hline Main Diodes & $2(M-1)$ & $2(M-1)$ & $2(M-1)$ \\
\hline Clamping Diodes & $(M-1) \times(M-2)$ & 0 & 0 \\
\hline dc-Bus Capacitors & $(M-1)$ & $(M-1)$ & 0 \\
\hline Balancing Capacitors & 0 & $(M-1) \times(M-2) / 2$ & 0 \\
\hline
\end{tabular}

\subsection{DC-DC Converters for DG Applications}

Dc-ac power converters (inverters) play key roles in connecting sustainable energy (SE) sources, and energy storage (ES) devices, to local loads and the utility grid. The power electronic interface circuits of the DG units perform several important tasks in order to make the output power of the SE sources and ES devices adequate for electric appliances. Typically, the first task is to boost the dc input voltage to a desired (rated) voltage level and invert the dc voltage to an ac voltage with a fixed frequency and amplitude. In the case of photovoltaic (PV) systems, it is desirable to keep the number of PV panels as low as possible. This means that the amplitude of the generated dc voltage will be lower than the grid voltage (in grid-connected mode) or the desirable load voltage (in stand-alone mode). Meanwhile, due to the nature of the ES sources, their generated power is usually varying by time. For instance, the output voltage and power of a PV panel vary in a wide range based on the operating conditions. Some of the affecting 
variables are incident solar radiation, ambient temperature, deposited dust and dirt on the panel surface, and the load current. The second task of the power electronic conversion system is to utilize the maximum available power of the SE sources. Besides the aforementioned functionalities, DG inverters have to meet some requirements like stability, efficiency, reliability, and power quality, which are common to all conversion systems. Several power electronic circuit topologies, along with the associated switching patterns and control algorithms, have been presented in literature, which perform the discussed tasks and address the aforementioned issues [1-22].

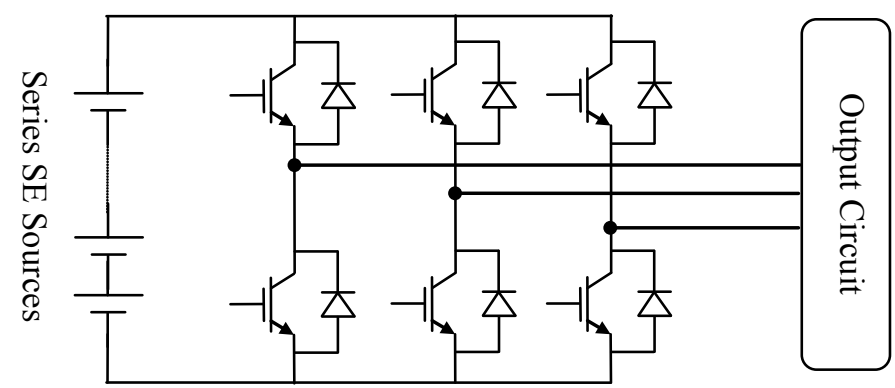

Figure 2.11: Basic single-stage power conversion system, consisting of series connected dc-sources

\subsubsection{Basic Single-Stage Conversion System}

A simple way to increase the output voltage of SE sources is to connect a number of them in series (to develop a high dc voltage without utilizing a boost circuit), and use a central pulsed width modulated (PWM) buck voltage source inverter (VSI) to produce an ac voltage [7] (see Figure 2.11). However, because of the high cost of PV panels, this series configuration seems as an expensive solution. In addition, putting a number of components in series usually lowers the reliability of the system. This is because the failure of one of the panels, or outage of the inverter, results in a complete loss of 
generation [7]. Moreover, mismatch of the series PV panels results in a reduction in the utilized energy, which can be interpreted as low efficiency [1].

\subsubsection{Conventional Two-Stage Conversion System}

In Figure 2.12, a step-up transformer is utilized at line-frequency to boost the output voltage of the PWM buck inverter. This well-known topology is robust and relatively efficient and reliable. Additionally, it can be used in module-integrated (or module-oriented) applications, which provides a higher flexibility than the topology of Figure 2.11. Besides that, each module has its own maximum power point tracking (MPP) system that increases the overall energy utilization. Its plug-and-play feature is also attractive, in which a complete PV system is achieved at a low investment cost [7]. On the other hand, this solution has some disadvantages, such as huge size, loud acoustic noise, and relatively high cost. Furthermore, the transformer should be designed for a relatively wide range of power, which leads to a low system efficiency. Overall, this topology is regarded as a poor solution [1].

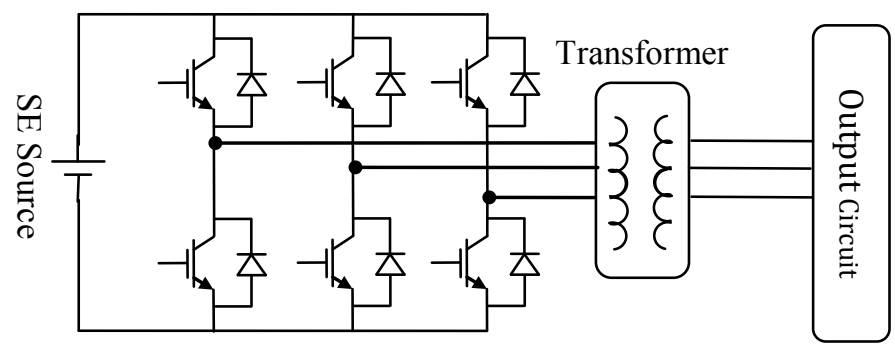

Figure 2.12: Transformer-based two-stage conversion system 


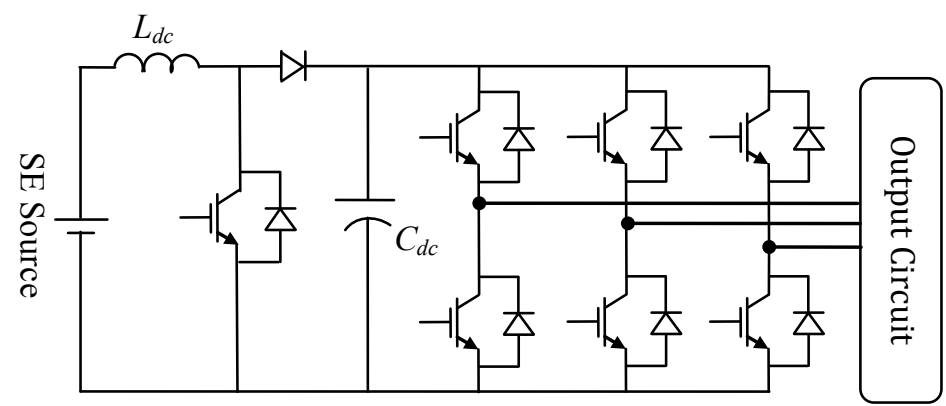

Figure 2.13: Transformer-less two-stage conversion system, consisting of a dc-dc boost converter cascaded with a PWM buck VSI

Transformer-less two-stage power conversion systems are one of the most commonly used interface circuits in SE-based DG applications. As can be seen in Figure 2.13, in this two-stage conversion topology, a dc-de converter and an inverter are cascaded. In the first stage, the dc-dc converter boosts and regulates the dc-bus voltage at a fixed level, and in the second stage, the PWM buck VSI converts the regulated dc voltage into an ac voltage. Since this topology does not contain a transformer, it has less magnetic components and higher efficiency. In comparison with the topology displayed in Figure 2.12, this topology requires two individual control systems and uses one more solid-state switch, as well as an electrolytic capacitor bank at the dc-bus. This will result in a lower reliability (and perhaps efficiency), and a more complicated control scheme [7].

\subsubsection{Multilevel Inverter}

Three-phase multilevel inverters can also be employed to interface the output of several SE sources with the grid $[2,8]$. Figure 2.7 presents the circuit diagram for the 
connection of a generic SE source to the output circuit by means of a diode-clamped three-level inverter. The SE source and additional components are represented as a voltage source, connected to the dc-side of the inverter. Like most inverters, multilevel inverters are connected to a grid through an inductive filter. A general model for the three-level inverter is presented in [9-10]. This model describes the dynamics of the dcand ac-sides, including the dynamics of dc and ac neutral points. Although the multilevel inverters are effective for this application, the added complexity of the circuit and the additional components reduce both the overall efficiency, and reliability of the system, and may raise the overall cost of the power electronic interface [2].

\subsubsection{Z-Source Inverter}

The z-source inverter has the capability of boosting and inverting the dc voltage in a single stage, with fewer solid-state switches in comparison with the multilevel inverters and the above-mentioned two-stage topologies depicted in Figures 2.13 and $2.14[3]$

The z-source inverter is a combination of a voltage source inverter, and a current source inverter. The circuit diagram of this inverter topology is shown in Figure 2.14. This inverter topology transforms the dc input voltage into the grid voltage, while the dc voltage can be above or below the grid voltage. The z-source inverter contains relatively high input current ripples, which may cause high stresses on the dc-link inductors and capacitors [12]. The application of this topology was reported by [13-15] as a gridconnected single-stage inverter for distributed generation systems, specialized for residential PV applications. 


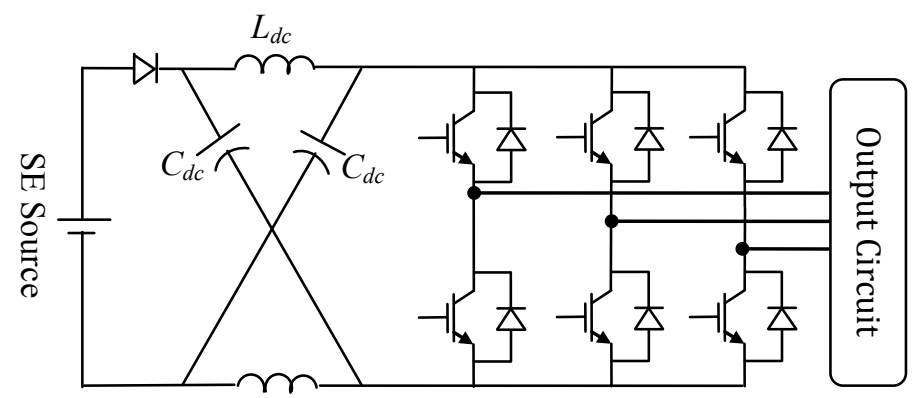

Figure 2.14: Single-stage Z-source inverter

\subsubsection{Single-Stage Boost Inverter}

In the last several decades, current source inverters have been replaced with voltage source inverters in many industrial applications. However, the CSI topology in conjunction with an appropriate control scheme can form a single-stage boost inverter that can be used for SE conversion systems. Several investigations have been recently reported on the application of CSIs as single-stage boost inverters for SE conversion systems [16-19]. As can be seen in Figure 2.15, the current source inverter utilizes a series inductor at the dc-link and a capacitor bank at the ac-side. In [4], a new switching pattern has been proposed for the CSIs in order to boost and invert the de voltage to a fixed voltage magnitude and fixed frequency for both stand-alone and grid-connected conditions. Besides the simplicity of the control scheme, elimination of dc-bus electrolytic capacitors is one of the greatest advantages of the single-stage boost inverter over the conventional two-stage converter, the multilevel inverter, and the Z-source inverter. This will significantly improve the reliability of the overall system, particularly in photovoltaic (PV) energy conversion systems, in which the mean-time-to-the-first- 
failure of inverters is about 5 years and the average lifetime of PV panels is about 20 years [6]. These attractive features have turned the attention of some of the latest research studies in the field of power electronics, to modeling and control of single-stage boost inverter [20-22]. This topology, along with a modified space-vector pulse-widthmodulation (SVPWM) technique, the so-called phasor pulse-width-modulation (PPWM), is the core of this dissertation, which will be elaborated upon in Chapter 4, and its statespace-averaged model will be derived in Chapters 5 and 6 .

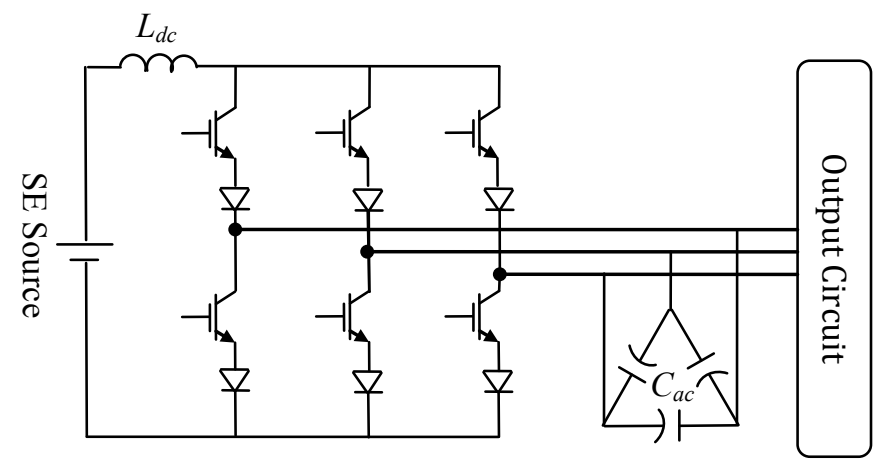

Figure 2.15: Single-stage current source boost inverter

\subsection{Summary}

Extensive research has been carried out on the related work to dc-ac power conversion systems, used in distributed generation applications. The most common converter topologies for grid-connected and stand-alone operations of sustainable energybased distributed generation system have been reviewed and compared. It should be mentioned that, besides the converter topologies reviewed in this chapter, the resonant converters are another major type of power electronic converters, which can be used for 
the same applications. However, since they are not related to the subject of this dissertation, they have not been discussed.

The next chapter provides a review of the existing techniques for steady-state and dynamic modeling of PWM converters. It includes the state-space averaging method and Park's transformation into rotating the frame of reference, which are necessary for accomplishing this dissertation. 


\section{CHAPTER 3}

\section{MODELING OF POWER ELECTRONIC CONVERTERS}

This chapter presents a review of the existing techniques for steady-state and dynamic modeling of power electronic converters. Moreover, the fundamentals of the states-space averaging technique and reference-frame theory, which will be used in Chapters 5 and 6 for dynamic modeling of the single-stage boost inverters, are provided.

This chapter contains seven sections. The steady-state modeling of dc-dc and dcac converters are discussed in Sections 1 and 2, respectively. Section 3 presents the statespace averaging technique. Dynamic modeling of $\mathrm{dc}-\mathrm{dc}$ and dc-ac converters are elaborated upon in Sections 4 and 5, respectively. The reference-frame theory is reviewed in Section 6, and Section 7 is the summary of the chapter.

\subsection{Steady-State Modeling of DC-DC Converters}

Similar to the existing topologies, numerous studies have been reported on the modeling and analysis of power electronic circuits in steady-state and dynamic conditions [23-58]. Under steady-state conditions, the voltage and current waveforms of any element in switching circuits can be found by the use of two basic principles; (1) inductor voltsecond balance and (2) capacitor amp-second or charge balance [24]. These concepts are particularly useful for analyzing dc-dc converters. For example, the inductor voltsecond balance can be used for calculating the voltage and current conversion ratios in a dc-dc boost converter [25]. The schematic diagram as well as inductor's current and voltage (over one switching cycle, $T_{S}$ ) of a boost converter are presented in Figure 3.1. 
The inductor volt-second principle implies that the hatched areas $A_{1}$ and $A_{2}$ are equal. As a result:

$V_{d c} D T_{s}=\left(V_{d c}-V_{o}\right)(1-D) T_{s}$

where, $0 \leq D<1$ denotes the duty ratio of the converter. The other parameters are as indicated on the circuit diagram. Manipulating this equation provides the voltage conversion ratio and the input-to-output transfer function of the dc-dc boost converter as [25]:

$\frac{V_{o}}{V_{d c}}=\frac{1}{1-D} \Rightarrow V_{o}=\frac{V_{d c}}{1-D}$

Accordingly, one can calculate the current conversion ratio in the same way.

(a)

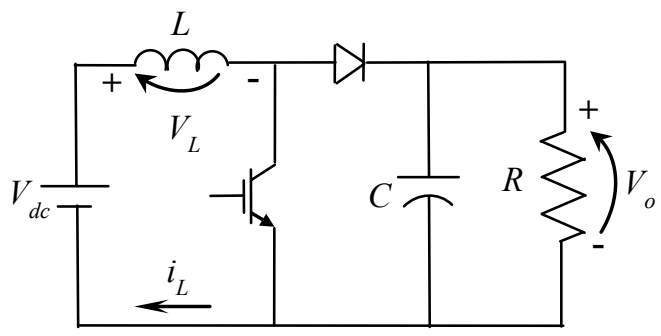

(b)

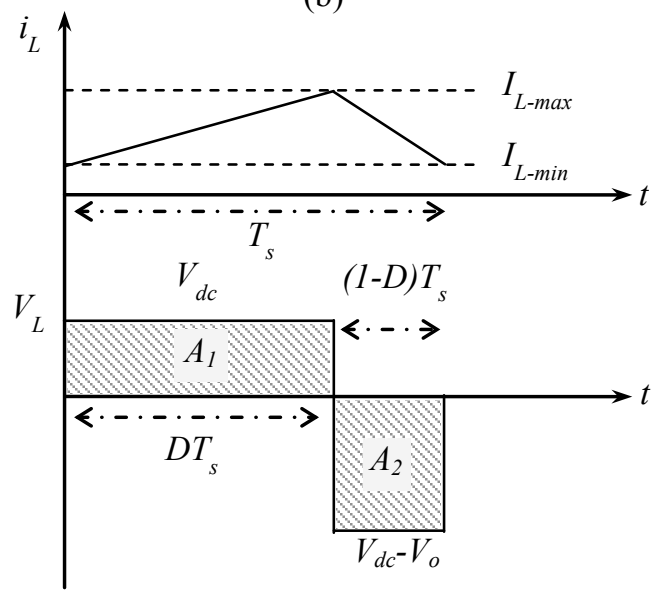

Figure 3.1: Inductor volt-second balance in a dc-dc boost converter, a) Schematic diagram of the boost converter, b) voltage and current profiles of the inductor under steady-state conditions

Although the system inputs, $V_{d c}$ and $D$ are assumed constants (invariant by time), the transfer function of (3.2) can be perturbed around its operating point in order to calculate (or approximate) the variations in the steady-state output of the system, $\Delta V_{o}$, in 
terms of small variations in the system inputs, i.e. $\Delta V_{d c}$ and $\Delta D$. For this purpose, it is required to replace $X \rightarrow X+\Delta X$, for $X=\left\{V_{d c}, D, V_{o}\right\}$, and substitute them in the transfer function (3.2), which is:

$V_{o}+\Delta V_{o}=\frac{V_{d c}+\Delta V_{d c}}{1-(D+\Delta D)}$

Replacing (3.2) in (3.3) and rearranging it, with the assumption of

$|D| \gg|\Delta D| \Rightarrow(1-D-\Delta D)(1-D) \approx(1-D)^{2}$

provides a linear relation between the variation of the system output and variations of its input:

$\Delta V_{o} \approx \frac{1}{(1-D)} \Delta V_{d c}+\frac{V_{d c}}{(1-D)^{2}} \Delta D$

It should be emphasized that (3.5) is not a dynamic equation. It is just showing how the steady-state operating point of the dc-dc boost inverter changes, if its inputs vary.

\subsection{Steady-State Modeling of DC-AC Converters}

Averaging-based methods have been widely used in literature for calculating the ac outputs of SPWM and SVPWM inverters under steady-state conditions [25-28]. These are discussed in the following subsections.

\subsubsection{Steady-State Model of SPWM Inverter}

The sinusoidal pulse-with-modulation (SPWM) technique has been elaborated upon in Chapter 2. The application of averaging in calculating the phase to neutral voltage of a three-phase SPWM voltage source inverter is presented in Figure 3.2, where 
$v_{t r i}$ and $v_{c, a}$ are the triangular voltage and control voltage for phase $a$, respectively, and $\widehat{V}_{t r i}$ denote the peak value of the triangular waveform. The average value of $v_{a n}$ over one switching cycle, $T_{s}$, can be can be written as follows:

$\bar{v}_{a n}\left(t, t+T_{s}\right)=\frac{1}{T_{S}}\left\{\left(1-\frac{v_{c, a}(t)}{\widehat{V}_{t r i}}\right) \frac{T_{s}}{2} \frac{V_{d c}}{2}-\left(1+\frac{v_{c, a}(t)}{\widehat{V}_{t r i}}\right) \frac{T_{s}}{2} \frac{V_{d c}}{2}\right\}=\frac{v_{c, a}(t)}{\widehat{V}_{t r i}} \frac{V_{d c}}{2}$

If the control voltage is defined as follows:

$v_{c, a}=m \widehat{V}_{t r i} \sin \left(2 \pi f_{1} t\right)$

where $m \geq 0$ is the modulation index of the PWM signals, and $f_{1}$ is the fundamental frequency of the desired output voltage, let us say $f_{1}=60 \mathrm{~Hz}$. Then, the fundamental component of $v_{\text {an }}$ will be obtained as:

$v_{a n 1}=m \frac{v_{d c}}{2} \sin \left(2 \pi f_{1} t\right)$

(a)

(b)

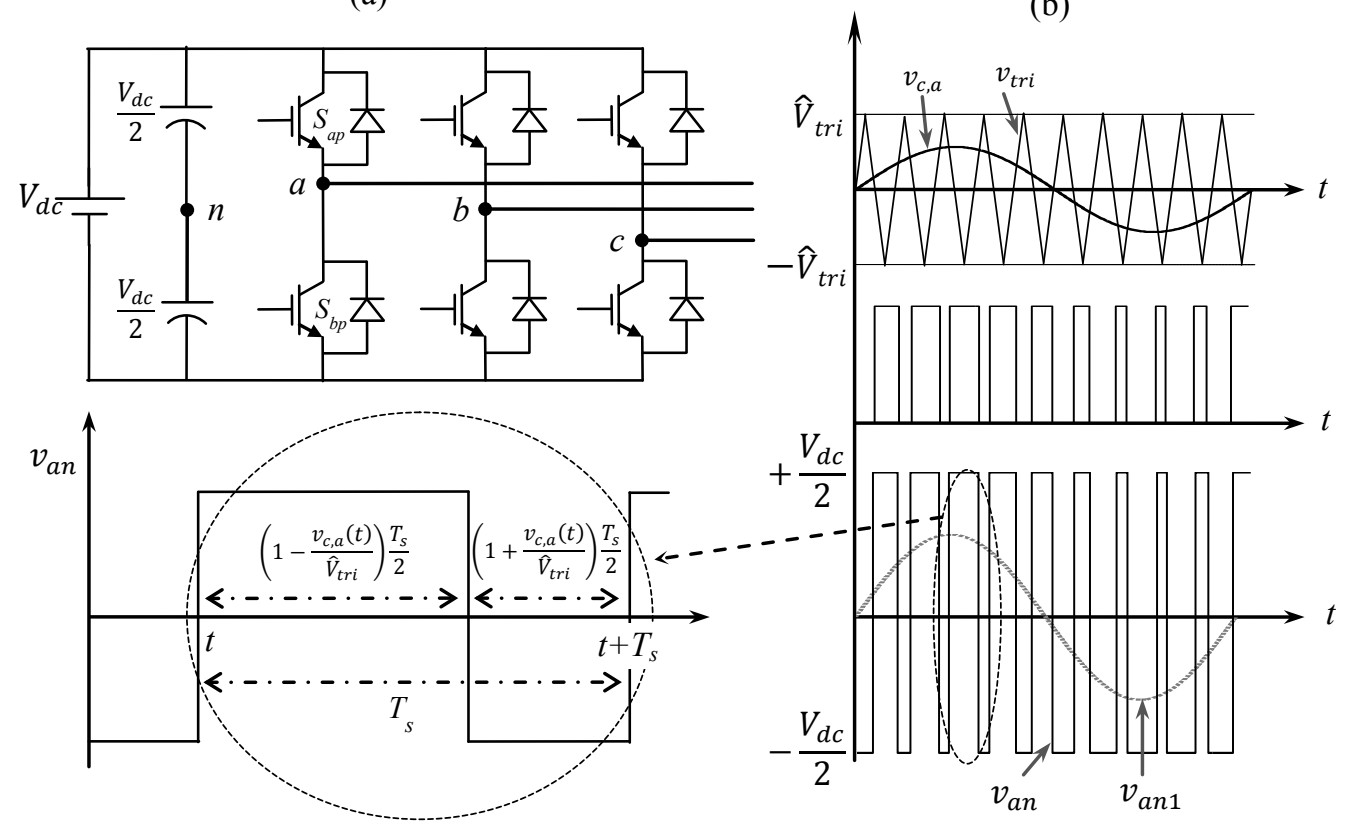

Figure 3.2: Steady-state modeling of a three-phase sinusoidal PWM inverter by averaging, a) schematic diagram of the inverter, b) PWM signals and the voltage between phase A and the neutral 
The same concept can be applied to derive the steady-state model of the threephase space-vector PWM voltage source inverter.

\subsubsection{Steady-State Model of SVPWM Inverter}

The space-vector pulse-width-modulation (SVPWM) and its fundamental equations have been discussed in the previous chapter. The application of averaging in calculating line-to-line voltage (between phases $a$ and $b$ ) of the three-phase SVPWM voltage source inverter in Sector (I) is presented. The methodology can be easily extended to other phases and sectors. Recalling from Chapter 2, the SVPWM inverter operates in three states in Sector (I), i.e. State 1 for the time-interval of $t_{1}=d_{1} T_{s}$, State 2 for the time-interval of $t_{2}=d_{2} T_{s}$, and State $0 / 7$ for the time-interval of $t_{0}=d_{0} T_{s}$, where $T_{s}=1 / f_{s}$ is the switching cycle and $d_{0}+d_{1}+d_{2}=1$. The corresponding line-to-line voltages, during each state, have been presented in Table 2.1. Using this table, the average value of $v_{a b}$ over one switching cycle can be can be calculated as follows:

$\bar{v}_{a b}\left(t, t+T_{s}\right)=\frac{1}{T_{s}}\left(t_{1} \times V_{d c}+t_{2} \times 0+t_{0} \times 0\right)=d_{1} V_{d c}$

Replacing $d_{1}$ from (2.10), and considering $\gamma=\omega t-\pi / 6$ (see Figure 2.6), the fundamental component of $v_{a b}$ can be obtained as:

$v_{a b 1}=\left|U^{r e f}\right| \cos \left(2 \pi f_{1} t\right)$

Similarly, the fundamental components of other line voltages can be calculated as follows:

$v_{b c 1}=\left|U^{r e f}\right| \cos \left(2 \pi f_{1} t-\frac{2 \pi}{3}\right)$
$v_{c a 1}=\left|U^{r e f}\right| \cos \left(2 \pi f_{1} t+\frac{2 \pi}{3}\right)$ 
which correspond to a balanced voltage output for the three-phase SVPWM inverter.

\subsection{State-Space Averaging Technique}

The dynamic model of the power electronic converters is needed to study the overall system stability, and to design controllers for the converter-based systems. Furthermore, the developed model can be linearized around any operating point in order to derive the state-space representation of the system, as well as the transfer functions associated with different inputs and outputs of the system. The model, in general, and the transfer function, in particular, can be used for many studies like frequency response analysis, stability, voltage regulation, seamless transition between stand-alone and gridconnected operational modes, filter design, and performing control studies of the system such as controllability and observability, nonlinear and intelligent control, and optimal and adaptive control. However, it should be noted that, because of their switching behavior and nonlinear elements, the analysis of power electronic converters is generally considered as a complicated task [23]. Averaging techniques are referred as the most common tools for dynamic modeling of the switching power converters [30-58]. The averaging methods have long been used for the analysis of nonlinear dynamical systems [29]. These techniques have been thoroughly discussed in the mathematical literature,

and the relationship between the original and averaged equations have been investigated [30-31]. In 1976, Middlebrook and Cuk bridged the gap existing between the state-space technique and the averaging method for modeling dc-dc power converters by introducing the state-space averaging method [32], which is an almost ideal compromise between accuracy and simplicity. 
The averaging technique is applied to the nonlinear dynamical systems, represented in the standard form [29] as given by:

$\dot{\boldsymbol{x}}=\epsilon \boldsymbol{f}(t, \boldsymbol{x}), \boldsymbol{x}\left(t_{0}\right)=\boldsymbol{x}_{0}$

where, $\boldsymbol{f}$ is a continuous function of $t$ and $\boldsymbol{x}$. The parameter $\epsilon$ plays the part of a small parameter, which characterizes the magnitude of certain perturbation. This parameter has a positive value; $0<\epsilon \leq \epsilon_{0}$ with $\epsilon_{0}>0$ a constant. Theorem of periodic averaging [29] states that the solution to the initial value problem of (1), $\boldsymbol{x}(t)$, can be approximated by $\widetilde{\boldsymbol{x}}(t)$, if $\boldsymbol{f}$ is periodic (or almost periodic [30]). Thus, one can write:

$\dot{\tilde{x}}=\epsilon \boldsymbol{F}(\widetilde{\boldsymbol{x}}), \tilde{\boldsymbol{x}}\left(t_{0}\right)=\boldsymbol{x}_{0}$,

where

$\boldsymbol{F}(\widetilde{\boldsymbol{x}})=\lim _{T \rightarrow \infty} \frac{1}{T} \int_{0}^{T} \boldsymbol{f}(\boldsymbol{x}, t) d t$

It should be emphasized that (3.13) can be referred as a time-invariant system, while (3.12) could be a time-varying equation. The theorem of general averaging has been briefly explained in Appendix A.

Accordingly, one can apply the averaging technique to a linear time-varying system that, in general, can be represented by the following state-space equation:

$\dot{\boldsymbol{x}}=\boldsymbol{A}(t) \boldsymbol{x}+\boldsymbol{B}(t) \boldsymbol{u}$

$\boldsymbol{y}=\boldsymbol{C}(t) \boldsymbol{x}+\boldsymbol{D}(t) \boldsymbol{u}$

where, for a $n$th order dynamical system with $r$ inputs and $m$ outputs: $\boldsymbol{x}$ is a $n \times 1$ vector (state vector), $\boldsymbol{u}$ is a $r \times 1$ vector (input vector), $\boldsymbol{y}$ is a $m \times 1$ vector (output vector), $\boldsymbol{A}$ is a $n \times n$ matrix (system matrix), $\boldsymbol{B}$ is a $n \times r$ matrix (control matrix), $\boldsymbol{C}$ is a $m \times n$ matrix (output matrix) and $\boldsymbol{D}$ is a $m \times r$ matrix [69]. 
This averaging technique provides a time-invariant approximate state-space equation for the system (3.15), the so-called state-space-averaged model:

$$
\begin{aligned}
& \dot{\tilde{x}}=\bar{A} \widetilde{x}+\bar{B} \boldsymbol{u} \\
& \dot{\tilde{y}}=\bar{C} \widetilde{x}+\bar{D} \boldsymbol{u}
\end{aligned}
$$

\subsection{Dynamic Modeling of DC-DC Converters}

Application of the so-called state-space averaging method for dynamic modeling of dc-dc converters can be best explained with an example. Figure 3.3 illustrates the application of the state-space averaging method to a dc-dc boost converter, operating in continuous conduction mode (CCM). Based on the state (ON and OFF) of the semiconductor switch (here an IGBT), the boost converter has two different linear circuits. Once, the switch is on, the converter operates in ON state for a time period of $d T_{s}$, where $T_{s}$ is the switching cycle and $d$ is the duty ratio of the converter. Accordingly, when the switch is off, the converter operates in OFF state for a time period of $(1-d) T_{s}$. According to the principles of electric circuits, each linear circuit can be represented by a state-space model, in which the independent inductor currents and capacitor voltages are usually selected as the state variables, and the voltage and current sources form the input vector to the model. Hence, the boost converter has two distinct state-space equations for the ON and OFF states. If the state vector is defined as $x=\left[i_{L}, v_{c}\right]^{T}$, where the superscript $T$ denotes the matrix transpose, the associated system and input matrices can be written as: 


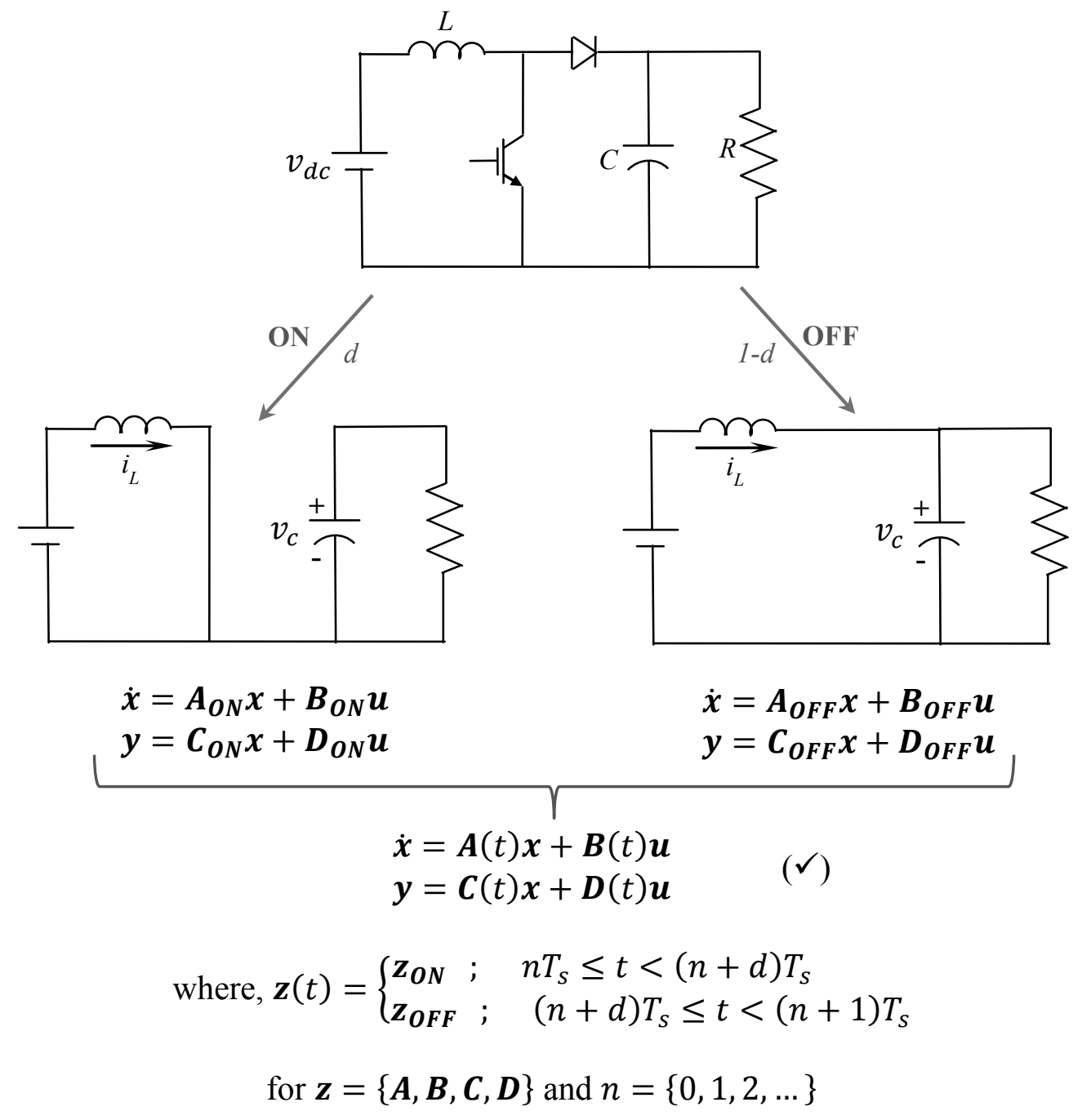

State-space averaging says:

$(\checkmark)$ can be approximated by the following state-space-averaged model

$$
\begin{aligned}
& \dot{\tilde{x}}=\bar{A} \widetilde{\boldsymbol{x}}+\bar{B} \boldsymbol{u} \\
& \widetilde{\boldsymbol{y}}=\overline{\boldsymbol{C}} \widetilde{\boldsymbol{x}}+\bar{D} \boldsymbol{u}
\end{aligned}
$$

where,

$$
\begin{array}{ll}
\overline{\boldsymbol{A}}=d \boldsymbol{A}_{\text {ON }}+(1-d) \boldsymbol{A}_{\text {OFF }} & \overline{\boldsymbol{C}}=d \boldsymbol{C}_{\text {ON }}+(1-d) \boldsymbol{C}_{\text {OFF }} \\
\overline{\boldsymbol{B}}=d \boldsymbol{B}_{\text {ON }}+(1-d) \boldsymbol{B}_{\text {OFF }} & \overline{\boldsymbol{D}}=d \boldsymbol{D}_{\text {ON }}+(1-d) \boldsymbol{D}_{\text {OFF }}
\end{array}
$$

Figure 3.3: Concept of the state-space averaging method, applied to a dc-dc boost converter, operating in continuous conduction mode (CCM) 


$$
\begin{array}{cc}
\boldsymbol{A}_{\text {ON }}=\left[\begin{array}{cc}
0 & 0 \\
0 & \frac{-1}{R C}
\end{array}\right] & \boldsymbol{B}_{\text {ON }}=\left[\begin{array}{c}
\frac{1}{L} \\
0
\end{array}\right] \\
\boldsymbol{A}_{\text {OFF }}=\left[\begin{array}{cc}
0 & \frac{-1}{L} \\
\frac{1}{C} & \frac{-1}{R C}
\end{array}\right] & \boldsymbol{B}_{\text {OFF }}=\left[\begin{array}{c}
\frac{1}{L} \\
0
\end{array}\right]
\end{array}
$$

This equation indicates that the overall state-space model is varying by time. In other words, a dc-dc boost converter, in CCM, is a time-varying system.

The goal of the so-called, state-space averaging method is to approximate the time-varying state-pace model of the boost converter by a time-invariant state-space model, called state-space-averaged model. This can be achieved through averaging the state-space equations of the converter over one switching cycle $T_{s}$. Accordingly, the statespace averaged model of the dc-dc boost converter, in CCM, is obtained as:

$\frac{d}{d t}\left[\begin{array}{l}\tilde{i}_{L} \\ \tilde{v}_{c}\end{array}\right]=\left[\begin{array}{cc}0 & \frac{d-1}{L} \\ \frac{1-d}{C} & \frac{-1}{R C}\end{array}\right]\left[\begin{array}{l}\tilde{i}_{L} \\ \tilde{v}_{c}\end{array}\right]+\left[\begin{array}{c}\frac{1}{L} \\ 0\end{array}\right] v_{d c}$

Suppose now that the duty ratio changes by time, that is, $d(t)=D+\delta d$, where $D$ is the steady-state (dc) duty ratio and $\delta d$ is a superimposed (ac) variation. The variation in the duty ratio corresponds to variations in the state variables of the system as $\widetilde{\boldsymbol{X}}=\widetilde{\boldsymbol{X}}+$ $\delta \widetilde{\boldsymbol{x}}$, where $\widetilde{\boldsymbol{X}}=\left[\tilde{I}_{L}, \tilde{V}_{c}\right]^{T}$ and $\delta \boldsymbol{x}=\left[\delta \tilde{i}_{L}, \delta \tilde{v}_{c}\right]^{T}$. Assuming $v_{d c}=v_{d c}+\delta v_{d c}$ and $\left|\frac{\delta d}{D}\right| \ll 1, \quad\left|\frac{\delta \tilde{x}}{X}\right| \ll 1$ and $\left|\frac{\delta v_{d c}}{v_{d c}}\right| \ll 1$

and

$$
\dot{\tilde{X}}=\left[\begin{array}{cc}
0 & \frac{D-1}{L} \\
\frac{1-D}{C} & \frac{-1}{R C}
\end{array}\right]\left[\begin{array}{c}
\tilde{I}_{L} \\
\tilde{V}_{c}
\end{array}\right]+\left[\begin{array}{c}
\frac{1}{L} \\
0
\end{array}\right] V_{d c}=0
$$


which is true in steady-state condition, and neglecting the higher-order nonlinear terms (that include products of ac variation $\delta d, \delta \widetilde{\boldsymbol{x}}$, and $\delta v_{d c}$ ), (3.18) can be rearranged as:

$\frac{d}{d t}\left[\begin{array}{l}\delta \tilde{i}_{L} \\ \delta \tilde{v}_{c}\end{array}\right]=\left[\begin{array}{cc}0 & \frac{D-1}{L} \\ \frac{1-D}{C} & \frac{-1}{R C}\end{array}\right]\left[\begin{array}{l}\delta \tilde{i}_{L} \\ \delta \tilde{v}_{c}\end{array}\right]+\left[\begin{array}{cc}\frac{1}{L} & \frac{V_{c}}{L} \\ 0 & \frac{-I_{L}}{C}\end{array}\right]\left[\begin{array}{c}\delta v_{d c} \\ \delta d\end{array}\right]$

The obtained linearized small-signal (ac) model considers the duty ratio as an input to the CCM dc-dc boost converter system.

A broad application of the state-space averaging technique, and its derivatives, for dynamic modeling and analysis of dc-dc converters, has been reported in literature [3347]. Nevertheless, it is worth mentioning that state-space averaging is not the only available tool in dealing with these type of problems, for instance [37] has proposed a Lagrangian approach for average modeling of PWM controlled dc-dc converters.

The theorem of general averaging [29] has been briefly explained in Appendix A. The mathematical background of the state-space averaging technique, applied for power electronic circuits, has been presented in [30]. It has been also shown that the averaging theory provides direct techniques for recovering oscillatory effects, such as ripples, from the averaged model.

\subsubsection{Linear Equivalent Circuits for Nonlinear PWM Converters}

The duty of power conversion systems is to convert electrical power from one voltage, current, or frequency level to another. This function is to be achieved with, ideally $100 \%$ efficiency together with adjustability of the conversion ratio. Switches, capacitors, and magnetic devices are the allowable elements, while the resistors, because of their adverse effect on the system efficiency, are usually avoided. However, this may 
lead to undesirable reductions in the damping of such systems, with consequent increased difficulty in design of the feedback loop and dynamic control [38]. Knowledge of the transfer functions is essential for design, and since the switching power converters are nonlinear, an appropriate modeling approach is needed. This emphasizes the motivation of modeling the nonlinear power converters by equivalent linear circuits, based on the physical interpretation of the corresponding state-space-averaged models.

In [38], a small-signal method has been used to derive equivalent linear circuits for nonlinear PWM switched-mode converters. These equivalent circuits can be used for the analysis of any electric system that includes these converters. The equivalent linear circuit of a dc-dc boost converter, in CCM, is shown in Figure 3.4.

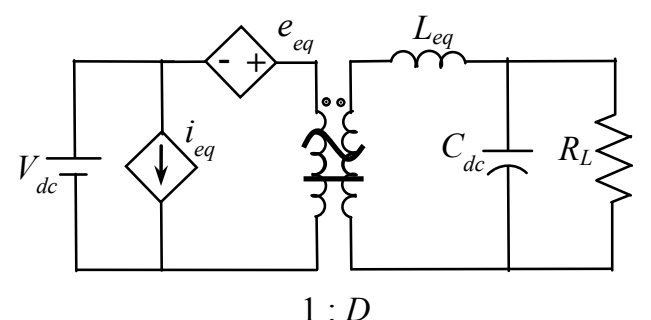

Figure 3.4: An equivalent linear circuit for the dc-dc boost converter in CCM

\subsubsection{Linear Equivalent Circuits for Switch-Diode Combinations}

As an alternative to developing an equivalent linear circuit for each individual power electronic converter, it is possible to derive three-terminal equivalent circuits for different combinations of a switch and a diode [39-40]. This can significantly reduce the number of required equivalent circuits, because unlike the countless number of existing power electronic converter topologies, only a few switch-diode combinations are used in 
their circuitries. The linear equivalents are specifically useful for analyzing large electric networks with huge number of switching power electronic converters, in which solving the nonlinear equations of the network in time domain becomes a tedious task. In these situations, linear equivalents can extremely reduce the computational effort and time. The three-terminal linear equivalent circuit for the switch-diode combination of a dc-dc boost converter has been presented in Figure 3.5 [39].

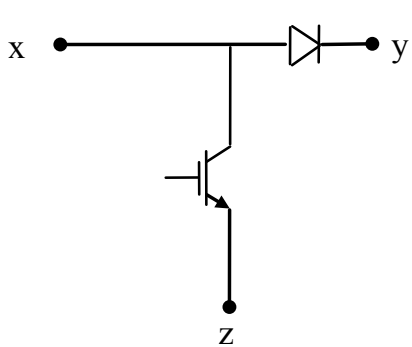

(a)

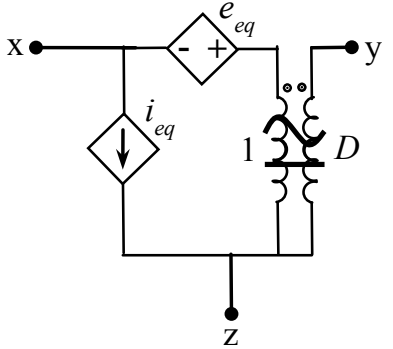

(b)

Figure 3.5: Three-terminal equivalent circuit for a switch-diode combination used in dc-dc boost converters, a) switch-diode combination, b) the equivalent three-terminal circuit

\subsubsection{Modeling under Non-Small Ripple Conditions}

The method of state-space averaging is successfully applied to PWM converters, but the conditions for the justification of state-space averaging are generally characterized by a small-ripple condition, and by a linear ripple approximation [32]. With the small-ripple approximation, the assumption is that a Fourier series expansion for a finite length segment of a circuit waveform should be dominated by its dc term. The linear ripple approximation requires that the circuit waveforms appear to be linear functions of time when examined over a time interval in between switch instances [41]. A 
more general averaging procedure that encompasses state-space averaging and is potentially applicable to a much broader class of circuits and systems has been considered in [41]. The proposed averaging scheme can be applied, in principle, to any periodically (or nearly periodically) driven system. In particular, the technique is shown to be effective on a number of resonant type converters, e.g. series resonant converter with voltage source load and dc-dc series resonant converter with capacitor load. Besides the resonant type converters, it has been shown that the refined (or generalized) statespace averaging method is able to consider the ripple component in PWM converters with large ripples, or relatively low switching frequency. However, it should be noted that adding each frequency component to the generalized state-space model, doubles the order of the model, which means that the refinement is achieved at the expense of complexity [42]. In another attempt to analyze dc-dc converters with a high ripple content (besides the generalized state-space averaging), new models have been derived in [43] to model the maximum and minimum envelopes (upper and lower bounds) of the output waveforms for dc-dc switching converters.

\subsubsection{Converter Analysis in Discontinuous Modes}

The most common operating mode of PWM converters is the continuous conduction mode (CCM), in which two networks are repeatedly switched by the action of power semiconductor switches. However, it is well known that CCM is not the only possible operating mode. As can be seen in Figure 3.3, there are two networks that are periodically switched in a dc-dc boost converter operating in the CCM, while there are three networks in the discontinuous conduction mode (DCM). The third possible 
network, the inductor discontinuous current mode (DICM) has been shown in Figure 3.6, in which the inductor is completely discharged and becomes open circuit. The discontinuous operation mode of PWM converters has been a subject of several studies in the field of dynamic modeling of power electronic converters [44-48]. For example in [44] the dc (large-) and ac (small-signal) analyses are applied to the basic PWM converter topologies. The small signal model is at the same order as the state-space-averaged model for the CCM and offers improved predictions of the low-frequency dynamics of PWM converters in DCM. Similarly [45] addresses the large-signal modeling and [46] proposes a numerical state-space averaging method for dynamic analysis of dc-dc converters in discontinuous mode of operation.

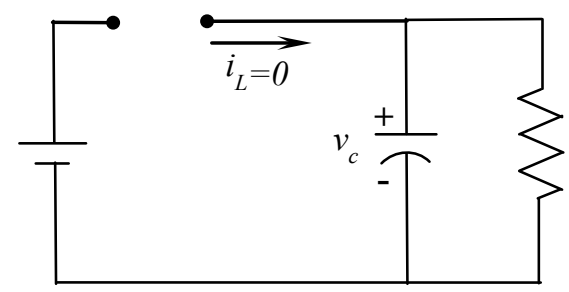

Figure 3.6: The third possible operating state, i.e. inductor discontinuous current mode (DICM), of a dc-dc boost converter under DCM. The other states have been already presented in Figure 3.3.

\subsection{Dynamic Modeling of DC-AC Converters}

The methods, described in the earlier sections, require thorough knowledge of all switched networks in all switching cycles. They are easier to use if there are only few switched networks to sketch out, and if these networks are the same for all switching cycles. In PWM ac converters (rectifiers, inverters, and cycloconverters) however, the 
number of switched topologies is large and increases rapidly with the number of phases. For a given switching modulation strategy, the switched networks in any switching cycle can differ from those in any other cycle. Therefore, an effective method to characterize low frequency behavior of PWM converters should avoid getting into topological switching details. It should allow derivation of converter dynamic equations by inspection of the converter as a whole and by use of familiar electrical principles [49].

\subsubsection{General Methodology for Modeling Inverters}

The applications of averaging methods have been also extended to modeling, analysis, and control of three-phase rectifiers and inverters [49-58]. A general methodology for deriving state-space equations for PWM dc-dc converters, inverters, rectifiers and cycloconverters has been developed in [49]. This methodology, which can characterize the low frequency components of the state variables and output waveforms of the switching circuits, are briefly described in this section. The concept has been explained for the phase $a$ of the current source inverter (CSI) in Figure 3.7.

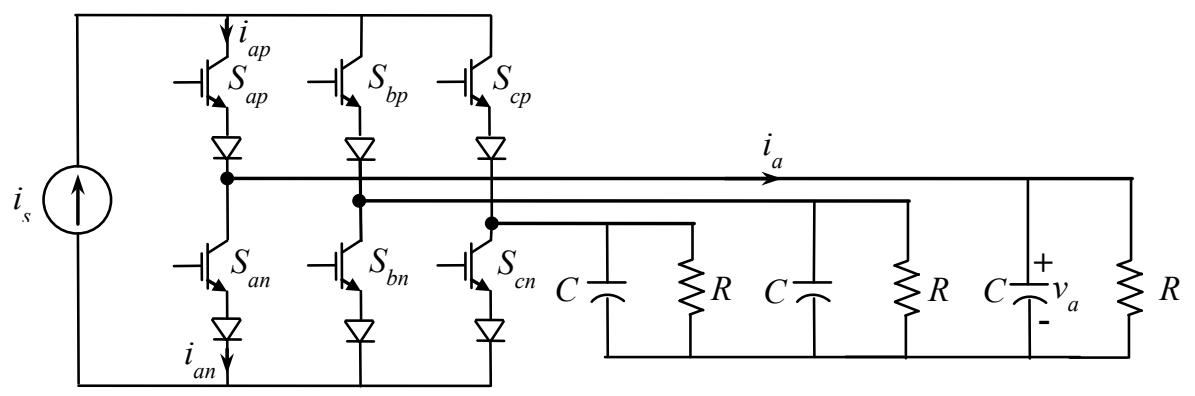

Figure 3.7: Current source inverter (CSI) 
The currents $i_{a p}, i_{a n}$, and $i_{a}$ can be written in term of the source current, $i_{s}$, and the gate signals as follows:

$\left\{\begin{array}{l}i_{a s}=s_{a p} i_{s} \\ i_{a n}=s_{a n} i_{s}\end{array} \Rightarrow i_{a}=\left(s_{a p}-s_{a n}\right) i_{s}\right.$

where, $s_{a p}$ and $s_{a n}$ are binary gate signals to the switches $S_{a p}$ and $S_{a p}$, respectively. Accordingly, the dynamic equation of phase $a$ can be written as:

$C \frac{d v_{a}}{d t}=\left(s_{a p}-s_{a n}\right) i_{s}-\frac{v_{a}}{R}$

The averaging method (see Appendix A) states that the dynamic equations can be approximated by replacement of the exact inputs and outputs by the low-frequency components of these terms, i.e.

$C \frac{d \tilde{v}_{a}}{d t}=d_{a} \bar{i}_{s}-\frac{\tilde{v}_{a}}{R}$

where, $d_{a}$ is the effective duty ratio, or just the duty ratio, of phase $a$ which is defined as:

$d_{a} \triangleq\left(\bar{s}_{a p}-\bar{s}_{a n}\right)$

Similarly, one can write the dynamic equations for the other phases and derive the statespace-averaged model of the CSI. The derived model can be represented by the equivalent linear circuit of Figure 3.8.
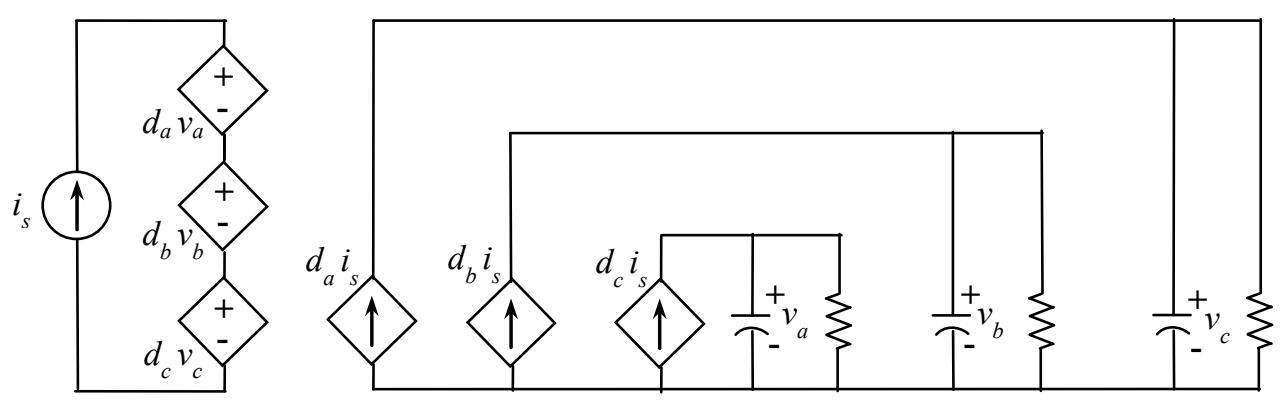

Figure 3.8: The equivalent linear circuit of the CSI of Figure 3.7 
Besides the general methodology, described in this section, an enormous number of studies have been carried out in the field of dynamic modeling and control of rectifiers and inverters [50-58]. A systematic approach for the small-signal modeling and control design of three-phase PWM rectifiers has been presented in [50-51]. The well-established design procedures used in dc-dc converter control design, have been adapted for the three-phase rectifier control based on the similarity in the small-signal dynamics of the three-phase rectifiers and their dc-dc counterparts. The approach is especially beneficial in a three-phase rectifier control, which is reduced to a single-input single-output system after closing the current loops. Finally, a wide-bandwidth output voltage control loop has been designed based on the obtained small-signal transfer function. A practical significance of these works is accounting the effects of digital control implementation and time-discrete nature of PWM, by adding one sampling period delay at the control inputs, and zero-order hold ( $\mathrm{ZOH})$ at the inductor currents' output. In a very recent work, a method for average-value modeling of the conventional three-phase (six-pulse) frontend rectifiers has been proposed in [52]. The system operation and dynamic performance of the developed average models have been demonstrated in discontinuous and continuous modes, as well as under balanced and unbalanced operation.

\subsubsection{Transformer-Based Equivalent Circuits}

In case of a system with a high number of reactive elements, [53] proposes to transform the nonlinear switching devices into equivalent linear ac networks by the use of equivalent time-varying transformers, and then deal with the system like a linear ac power system. The method has been applied to an eight-order system (a current source 
rectifier-inverter) and a buck-boost inverter, and promising results have been achieved. The time-varying-transformer-based equivalent circuit of the current source inverter of Figure 3.7 is presented in Figure 3.9; the parameters are defined in section 3.4.4.

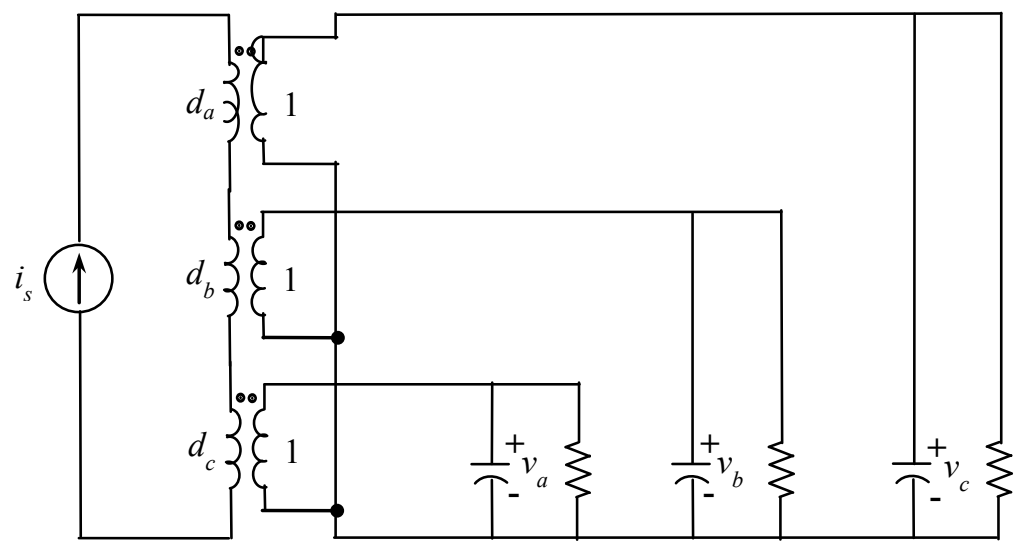

Figure 3.9: The time-varying-transformer-based equivalent circuit of the CSI of Figure 2.13

\subsubsection{Other Achievements}

A reduced-order small-signal model for a three-phase PWM rectifier has been developed in [54], where the three-phase rectifier was approximated by two parallel dcdc converters, each one valid for a $60^{\circ}$ line period interval. Consequently, it would be possible to use modeling and control techniques of dc-dc converters, which have been thoroughly studied and used in the field of power electronics. A three-phase boost rectifier has been modeled as two parallel dc-dc boost converters in Figure 3.10. This is over the $60^{\circ}$ line period interval, in which phase $a$ has the highest negative voltage. For other time intervals, the active topology includes different switch combinations, but the 
topological feature will be the same due to the symmetrical operation of three-phase converters.

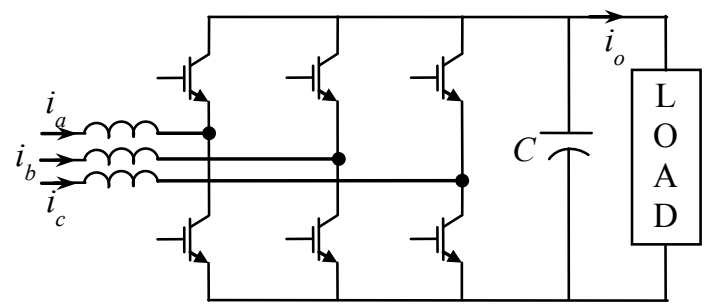

(a)

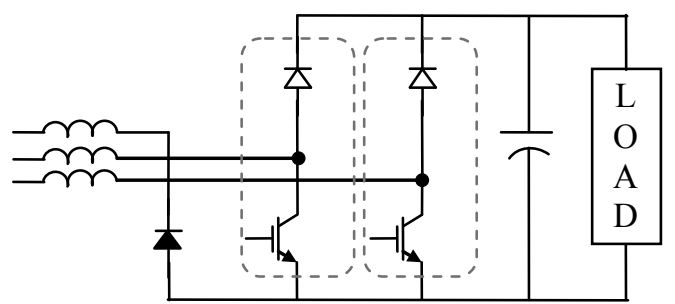

(b)

Figure 3.10: The concept of reduced order model, a) three-phase boost rectifier, b) active topology over the $60^{\circ}$ line period interval, in which phase $a$ has the highest negative voltage

In another attempt to model inverters, it has been shown in [55] that it is possible to develop the dynamic model of inverters in the phasor domain, which is readily compatible with the steady-state modeling and control techniques that are used to study ac power system dynamics. The dynamic phasors have been elaborated upon in [56].

It should be emphasized that the application of the state-space averaging method is not limited for the analysis of conventional VSI and CSI inverters, and it can be applied to other inverter topologies, such as multilevel [57] and Z-source [58] inverters. Moreover, once a converter is connected to a larger system, its model can be combined with the models of other subsystems of the larger system, in order to investigate the performance of the entire system. For instance, dynamic models of grid-connected inverters, as one of the major applications of the power electronic converters, have been 
extensively used in literature for investigation of the dynamic performance of large power systems in the presence of the grid-connected inverters [59-63].

\subsection{Reference-Frame Theory}

Transformation into rotating frames of reference is a common practice in the study and analysis of three-phase electric circuits. The direct-quadrature-zero (or $d q o-$ ) transformation, which was first introduced by Park in late 1920s [97], has been extensively used to reduce the complexities involved with analysis of three-phase electric machines, power systems, and power electronic inverters/rectifiers. This transformation refers the circuit variables to a frame of reference that rotates at an arbitrary angular velocity. In the case of balanced three-phase systems, the dqo-transformation into a synchronous frame of reference can reduce the three ac quantities to two dc quantities. This property can be particularly useful in dynamic and control studies of three-phase inverters. Accordingly, the Park's transformation into the synchronous frame of reference has been used in this dissertation for dynamic modeling and analysis of a newly proposed dc-ac converter (called single-stage boost inverter), which can convert a low dc voltage to a high ac voltage in a single-stage. Thus, a review of the reference-frame theory, and particularly $d q o$-transformation into synchronous frame of reference, is necessary in order to proceed with the later chapters, which present the contributions of this dissertation.

A change of variables that formulates a transformation of the three-phase variables of stationary circuit elements to arbitrary reference frame may be expressed as: 
$\left[\begin{array}{l}f_{q} \\ f_{d} \\ f_{o}\end{array}\right]=\frac{2}{3}\left[\begin{array}{ccc}\cos \left(\theta_{f}\right) & \cos \left(\theta_{f}-\frac{2 \pi}{3}\right) & \cos \left(\theta_{f}+\frac{2 \pi}{3}\right) \\ \sin \left(\theta_{f}\right) & \sin \left(\theta_{f}-\frac{2 \pi}{3}\right) & \sin \left(\theta_{f}+\frac{2 \pi}{3}\right) \\ \frac{1}{2} & \frac{1}{2} & \frac{1}{2}\end{array}\right]\left[\begin{array}{l}f_{a} \\ f_{b} \\ f_{c}\end{array}\right]$

where, $f$ can represent either voltage or current (and flux linkage or electric charge in general), and $\theta_{f}$ denotes the angular displacement of the rotating frame of reference, which may rotate at any constant or varying angular velocity or it may remain stationary. The change of variables may be applied to variables of any waveform and time sequence; however, it has been found that the transformation given above is particularly appropriate for an $a b c$ sequence [28].

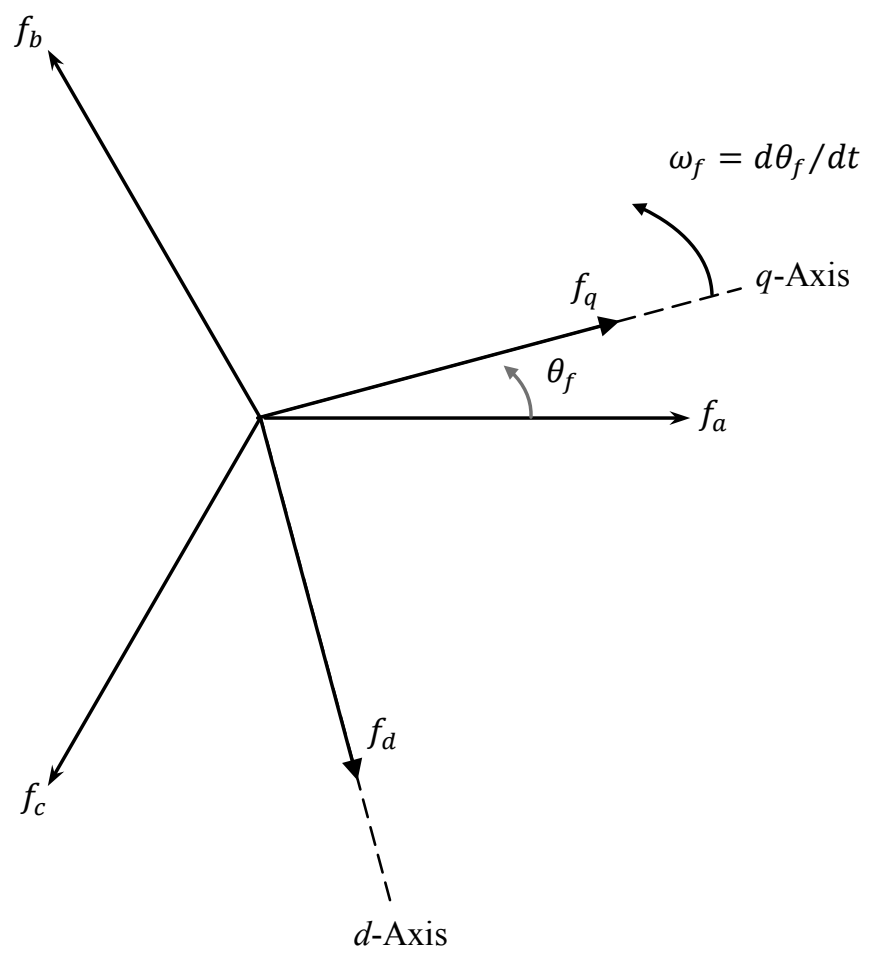

Figure 3.11: $d q$-transformation for three-phase circuit quantities portrayed by trigonometric relationships 
Although the transformation to the arbitrary reference-frame is a change of variables and needs no physical connotation, it is often convenient to visualize the transformation equations as trigonometric relationships between variables as shown in Figure 3.11 [28].

Like any linear transformation, the Park's transformation also has an inverse transformation that is:

$$
\left[\begin{array}{l}
f_{a} \\
f_{b} \\
f_{c}
\end{array}\right]=\left[\begin{array}{ccc}
\cos \left(\theta_{f}\right) & \sin \left(\theta_{f}\right) & 1 \\
\cos \left(\theta_{f}-\frac{2 \pi}{3}\right) & \sin \left(\theta_{f}-\frac{2 \pi}{3}\right) & 1 \\
\cos \left(\theta_{f}+\frac{2 \pi}{3}\right) & \sin \left(\theta_{f}+\frac{2 \pi}{3}\right) & 1
\end{array}\right]\left[\begin{array}{l}
f_{q} \\
f_{d} \\
f_{o}
\end{array}\right]
$$

Also, one can calculate the time-derivatives of the transformation and its inverse as:

$$
\begin{aligned}
\frac{d}{d t}\left[\begin{array}{l}
f_{q} \\
f_{d} \\
f_{o}
\end{array}\right]= & \frac{2}{3}\left[\begin{array}{ccc}
\cos \left(\theta_{f}\right) & \cos \left(\theta_{f}-\frac{2 \pi}{3}\right) & \cos \left(\theta_{f}+\frac{2 \pi}{3}\right) \\
\sin \left(\theta_{f}\right) & \sin \left(\theta_{f}-\frac{2 \pi}{3}\right) & \sin \left(\theta_{f}+\frac{2 \pi}{3}\right) \\
\frac{1}{2} & \frac{1}{2}
\end{array}\right] \cdot \frac{d}{d t}\left[\begin{array}{l}
f_{a} \\
f_{b} \\
f_{c}
\end{array}\right] \\
& +\frac{2}{3} \omega_{f}\left[\begin{array}{ccc}
-\sin \left(\theta_{f}\right) & -\sin \left(\theta_{f}-\frac{2 \pi}{3}\right) & -\sin \left(\theta_{f}+\frac{2 \pi}{3}\right) \\
\cos \left(\theta_{f}\right) & \cos \left(\theta_{f}-\frac{2 \pi}{3}\right) & \cos \left(\theta_{f}+\frac{2 \pi}{3}\right) \\
0 & 0 & 0
\end{array}\right]\left[\begin{array}{l}
f_{a} \\
f_{b} \\
f_{c}
\end{array}\right] \\
\frac{d}{d t}\left[\begin{array}{l}
f_{a} \\
f_{b} \\
f_{c}
\end{array}\right]= & {\left[\begin{array}{ccc}
\cos \left(\theta_{f}\right) & \sin \left(\theta_{f}\right) & 1 \\
\cos \left(\theta_{f}-\frac{2 \pi}{3}\right) & \sin \left(\theta_{f}-\frac{2 \pi}{3}\right) & 1 \\
\cos \left(\theta_{f}+\frac{2 \pi}{3}\right) & \sin \left(\theta_{f}+\frac{2 \pi}{3}\right) & 1
\end{array}\right] \cdot \frac{d}{d t}\left[\begin{array}{l}
f_{q} \\
f_{d} \\
f_{o}
\end{array}\right] } \\
& +\omega_{f}\left[\begin{array}{rrr}
-\sin \left(\theta_{f}\right) & \cos \left(\theta_{f}\right) & 1 \\
-\sin \left(\theta_{f}-\frac{2 \pi}{3}\right) & \cos \left(\theta_{f}-\frac{2 \pi}{3}\right) & 1 \\
-\sin \left(\theta_{f}+\frac{2 \pi}{3}\right) & \cos \left(\theta_{f}+\frac{2 \pi}{3}\right) & 1
\end{array}\right]\left[\begin{array}{l}
f_{q} \\
f_{d} \\
f_{o}
\end{array}\right]
\end{aligned}
$$




\subsection{Summary}

Extensive research has been carried out on the modeling of power electronic converters. Contributions of a variety of work, since 1976 to 2012, have been demonstrated and the prominent methodologies have been discussed in more detail. It has been shown that the averaging technique plays a key-role in the modeling of switch-mode power converters, and undoubtedly, the state-space averaging method is the most convenient tool for deriving the dynamic models of PWM power electronic converters. Finally, reference-frame theory, which is required for dynamic modeling of the recently proposed single-stage boost inverter, has been reviewed. The idea and fundamental equations of this inverter will be presented in the next chapter. 


\section{CHAPTER 4}

\section{SINGLE-STAGE BOOST INVERTER}

The idea of the recently proposed single-stage boost inverter (SSBI) is presented and discussed in this chapter. The circuit topology, switching pattern, control strategy, and steady-state performance of the single- and three-phase SSBIs are explained in detail and the concept is proved through simulation and experiments. This chapter is the backbone of this dissertation, and lays the groundwork for the later chapters, which are the main outcomes and contributions of this dissertation.

This chapter contains three sections. The three-phase SSBI is elaborated upon in Section 1. Section 2 is the extension of the work to the single-phase SSBI, and the summary is presented in Section 3.

\subsection{Three-Phase Single-Stage Boost Inverter}

In the past several decades, current source inverters have been replaced with voltage source inverters in many industrial applications. However, the CSI topology has the capability to be used as a SSBI. A single-stage CSI-based boost-inverter with a novel control strategy has been proposed in [4], which is suitable for sustainable energy (SE) systems (particularly PV and fuel-cell), and energy storage (ES) applications. The new method has been developed based on the concept of space-vector pulse-width-modulation (SVPWM). However, the developed switching pattern is formulated based upon phasor quantities, and not the space-vectors, so this modified SVPWM technique has been called phasor pulse-width-modulation (PPWM). Nevertheless, this strategy preserves all the 
advantages of an SVPWM technique such as simplicity, robustness, and ease of programming in digital processors.

\subsubsection{Circuit Topology}

Figure 4.1 shows the complete power circuit schematic of the three-phase boost inverter, where $V_{d c}$ is a dc voltage source (representing an SE source), $L_{d c}$ is the dc-link inductor, $C_{a c}$ represents the ac-side film capacitors, and $L_{a c}$ represents the line inductors.

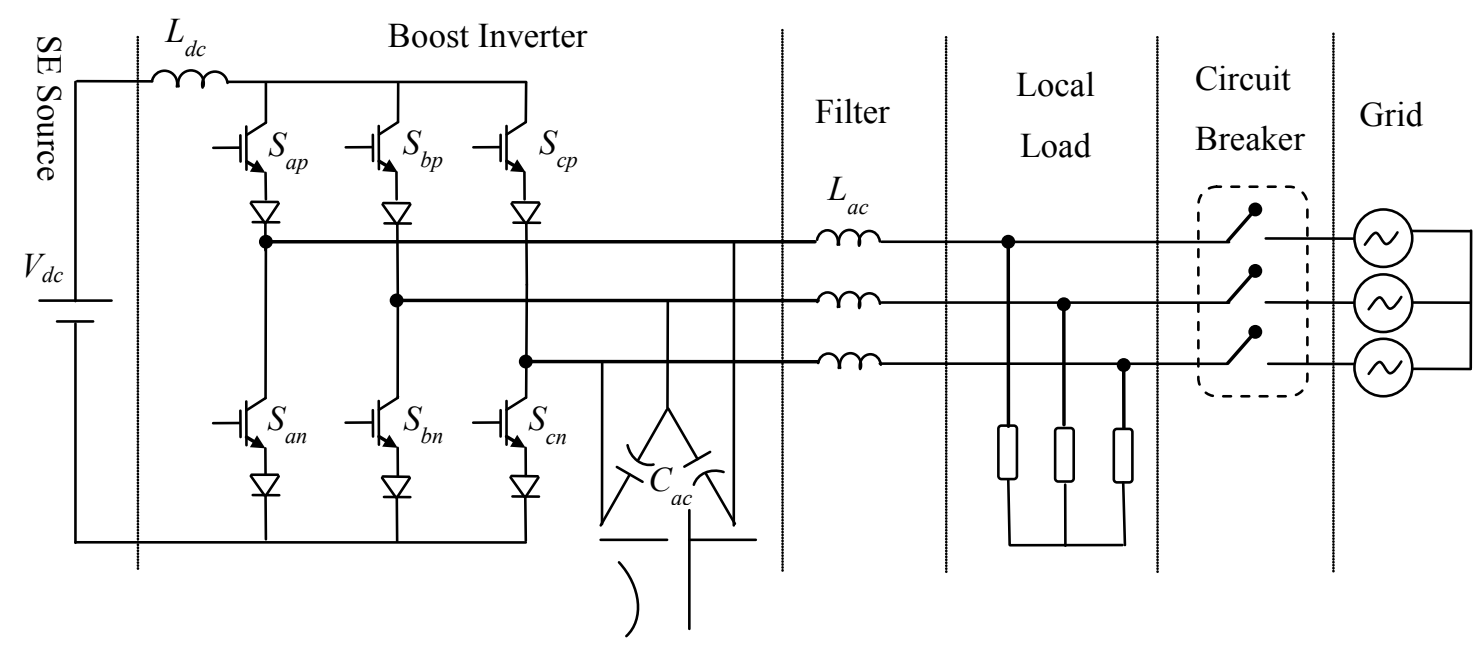

Figure 4.1: Complete power circuit schematic diagram of the CSI-based boost inverter

In this circuit topology, at any given instant, one of the upper switches and one of the lower switches are kept on. During the charging times the switches in a same leg are simultaneously on, so that the magnetic energy in $L_{d c}$ is increased to boost the output voltage and inject power to the output circuit. The switching pattern, which is utilized for the CSI-based boost inverter topology, will be elaborated upon with mathematical formulations later in this chapter. The PWM switching pattern and formulation developed 
herein is very close to the conventional SVPWM. However, in this pattern, the spacevectors of the currents and voltages are not applied in the development of the new formulations. It should also be emphasized that in the conventional space-vector formulation, the inverter switching time-intervals (or duty cycles) are in direct proportion to $V_{m} / V_{d c}$, where the time-intervals in the presented formulation for CSI-based boost inverters are in direct proportion to $V_{d c} / V_{m}$. Meanwhile, the six main switching states, and two zeros, with three switches conducting at any given instant in conventional SVPWM techniques, are adapted to six states with only two switches conducting at any given instant, as well as three charging states in the proposed/developed switching pattern for the CSI based boost inverter. These charging states are necessary in order to boost the dc input voltage. This control strategy is capable of being applied to both grid-connected and stand-alone modes of operation. The calculations for obtaining the control parameters will be presented in the following sections. During grid-connected operation of the SSBI, the output voltage is maintained by the grid, and the SSBI has to regulate the output current in a way that the delivery of power to the grid is controlled. In this situation, the SSBI operates in a current source, current regulated mode. In the second case, i.e. the stand-alone operation, the SSBI is connected to a local load (an RL load), and has to regulate the output voltage. While the SSBI is still a current-source, it operates in currentsource voltage-regulated mode.

\subsubsection{Switching Pattern}

The switching pattern, proposed in [4], for the three-phase single-stage current source boost inverter includes six sectors and nine states (three charging states and six 
discharging states) with only two switches conducting at any given instant. In other words, the six main switching states, and two zeros, with three switches conducting at any given instant in conventional SVPWM techniques, are modified to six states with only two switches conducting at any given instant, as well as three charging states in PPWM for current source single-stage boost inverter. The charging states are necessary in order to boost the dc input voltage. Again, it should be emphasized that the PPWM has been formulated based on the phasor quantities, and not the space-vectors.

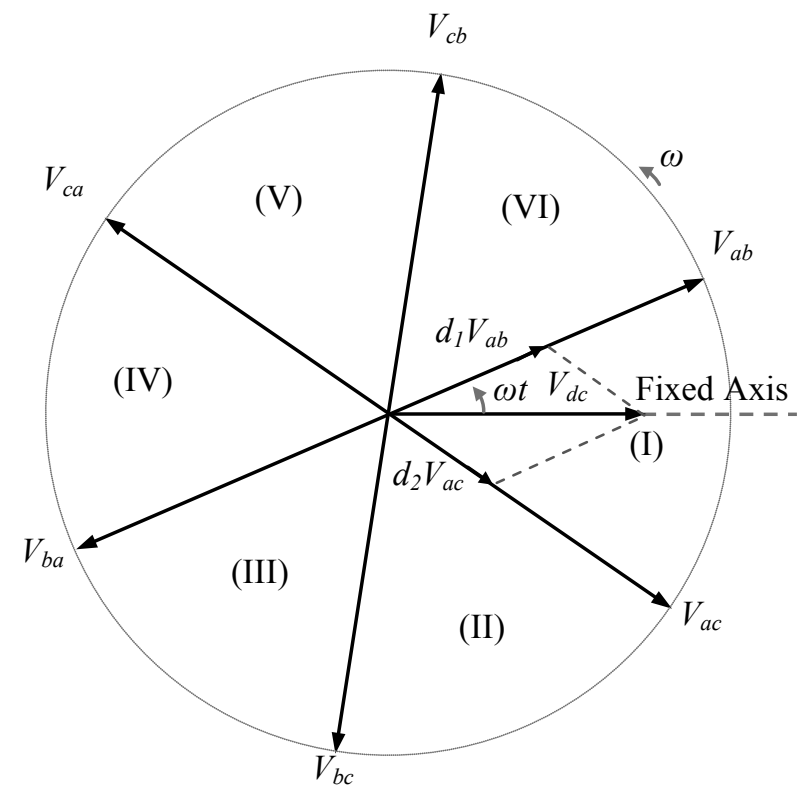

Figure 4.2: Line-to-line voltage phasors and the associated sectors

The six sectors, separated by six line-to-line voltage phasors, $V_{a b}, V_{a c}, V_{b c}, V_{b a}$, $V_{c a}$, and $V_{c b}$, are presented in Figure 4.2. As can be seen in this figure, the dc-source voltage, $V_{d c}$, is located in Sector (I) and the corresponding discharging and charging time-intervals can be calculated as shown in Figure 4.3. In each switching cycle, $T_{S}$, 
there are three time-intervals; one time-interval for charging the dc-link inductor, $t_{c}=d_{c} T_{s}$, and two time-intervals for injecting current into two different phases, $t_{d 1}=$ $d_{1} T_{s}$ and $t_{d 2}=d_{2} T_{s}$. For example, while Sector (I) is passing the X-axis in Figure $4.2, V_{d c}$ falls in between $V_{a b}$ and $V_{a c}$, this is when the voltage of phase $a$ is in its positive extreme. Thus, the proposed method uses phase $a$ in the entire sector to close the path of the load current, i.e. $S_{a p}$ stays on over this switching cycle. On the other hand, phase $b$ and phase $c$ are intermittently used for the negative part of the load current, see Figure 4.4. Thus, there are three states and consequently three time-intervals in Sector (I):

i) State C1: The charging time-interval, $t_{c}=d_{c} T_{s}$, in which two switches from leg $a$ are closed and the dc-link inductor is being charged, see I-C1 $\left(t_{c}\right)$ of Figure 4.4

ii) State D1: The first discharging time-interval, $t_{d 1}=d_{1} T_{s}$, where the inductor current is directed into phases $a$ and $b$. During this period of time, the upper-switch of leg $a, S_{a p}$, and the lower-switch of leg $b, S_{b n}$, of the inverter are closed, see I-D2 $\left(t_{d 1}\right)$ of Figure 4.4 .

iii) State D2: The second discharging time-interval, $t_{d 2}=d_{2} T_{s}$, where the inductor current is directed into phase $a$ and $c$. During this period of time, the upper-switch of leg $a, S_{a p}$, and the lower-switch of leg $c, S_{c n}$, of the inverter are closed, see I-D3 $\left(t_{d 2}\right)$ of Figure 4.4 .

It should be noted that within a given sector, one of the switches does not have to be switched at all, as shown in Figure 4.4. The six sectors along with the proper 
switching time-intervals are summarized in Table 4.1 for all six sectors. In this table, $V_{1}$ and $V_{2}$ denote the associated first and second voltage vectors in each sector in Figure 4.2. $S_{i p}$ and $S_{i n}$ represent the positive (upper) and negative (lower) switches in each leg $i=\{a, b, c\}$ of the inverter, see Figure 4.1 , and $t_{c}, t_{d 1}$, and $t_{d 2}$ indicate the time-intervals of the charging states, as well as, the two consecutive discharging states corresponding to each sector.

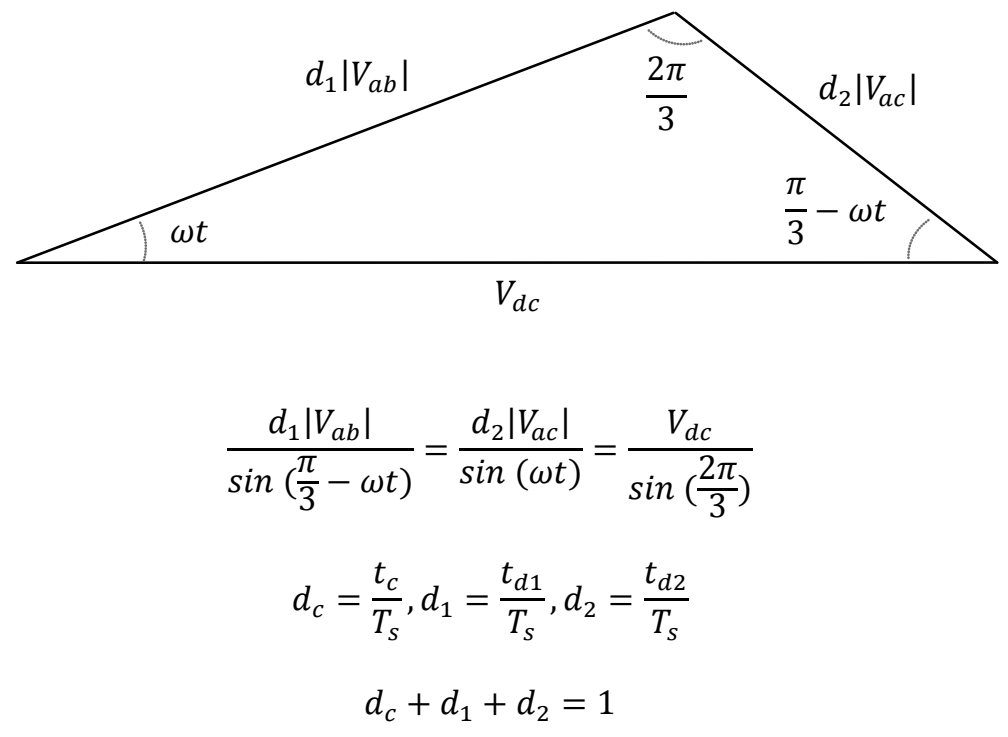

Figure 4.3: Sector (I), calculation of the discharging duty ratios, $d_{1}$ and $d_{2}$, as well as the charging duty ratio, $d_{c}$

Table 4.1: Sectors and switching states

\begin{tabular}{ccccccccc}
\hline Sector & $V_{1}$ & $V_{2}$ & $S_{a p}$ & $S_{a n}$ & $S_{b p}$ & $S_{b n}$ & $S_{c p}$ & $S_{c n}$ \\
\hline (I) & $V_{a b}$ & $V_{a c}$ & $T_{s}$ & $t_{c}$ & 0 & $t_{d 1}$ & 0 & $t_{d 2}$ \\
\hline (II) & $V_{a c}$ & $V_{b c}$ & $t_{d 1}$ & 0 & $t_{d 2}$ & 0 & $t_{c}$ & $T_{s}$ \\
\hline (III) & $V_{b c}$ & $V_{b a}$ & 0 & $t_{d 2}$ & $T_{s}$ & $t_{c}$ & 0 & $t_{d 1}$ \\
\hline (IV) & $V_{b a}$ & $V_{c a}$ & $t_{c}$ & $T_{s}$ & $t_{d 1}$ & 0 & $t_{d 2}$ & 0 \\
\hline (V) & $V_{c a}$ & $V_{c b}$ & 0 & $t_{d 1}$ & 0 & $t_{d 2}$ & $T_{s}$ & $t_{c}$ \\
\hline (VI) & $V_{c b}$ & $V_{a b}$ & $t_{d 2}$ & 0 & $t_{c}$ & $T_{s}$ & $t_{d 1}$ & 0 \\
\hline
\end{tabular}



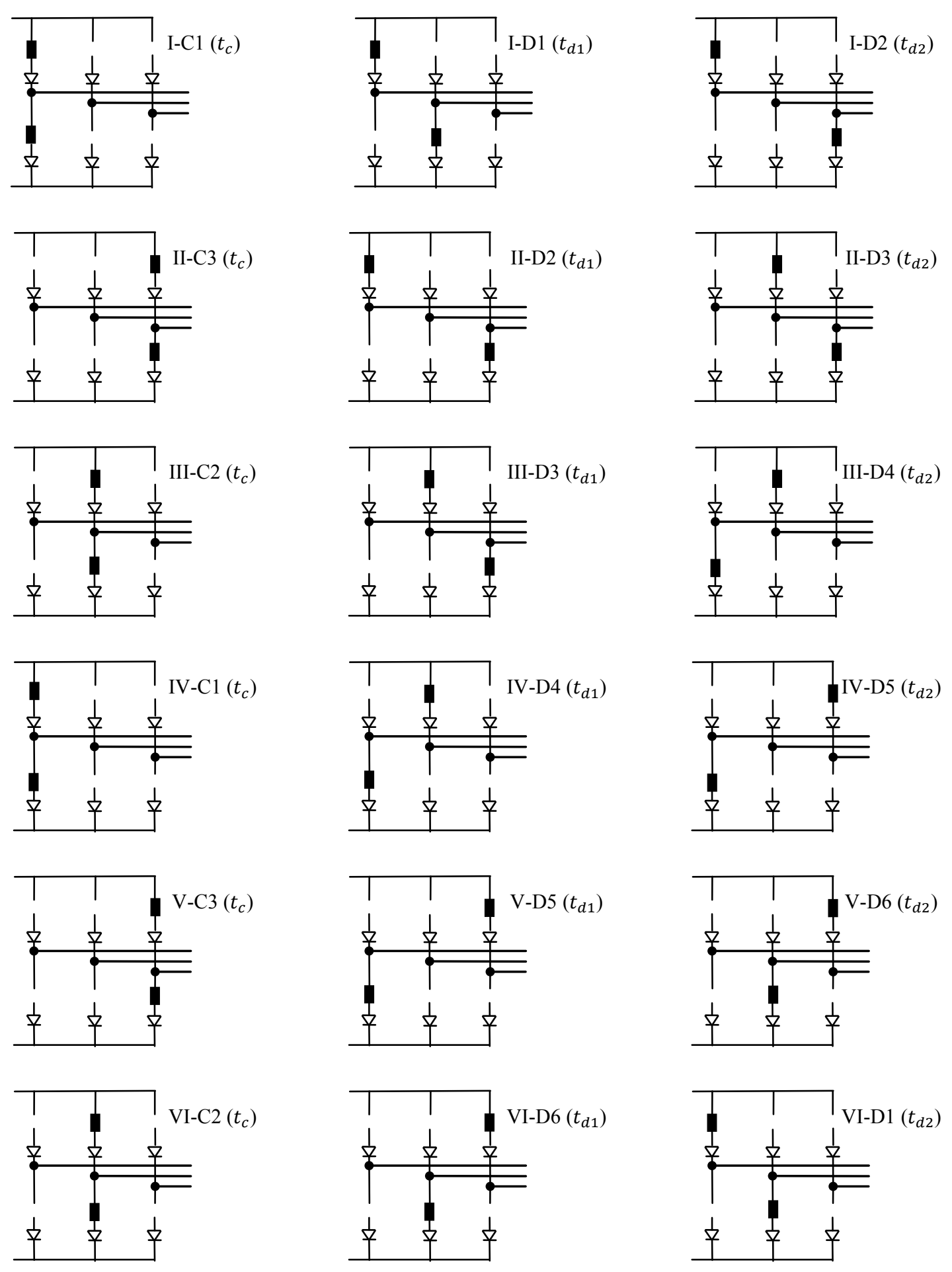

Figure 4.4: The charging (C1-C3) and discharging (D1-D6) states of operation for the SSBI 
Let us assume that the dc-link inductance, $L_{d c}$, and the dc input voltage, $V_{d c}$, stay constant over a switching cycle, $T_{s}$. Figure 4.5 shows the voltage and current waveforms of the dc-link inductor under this assumption. Using Figure 4.5, and considering the applied voltage across the dc-link inductor as $v_{L}=L_{d c} \Delta i_{d c} / \Delta t$, one can write the following equations for Sector (I), i.e. $0 \leq \omega t<\pi / 3$ (while the results can be easily extended to the other sectors):

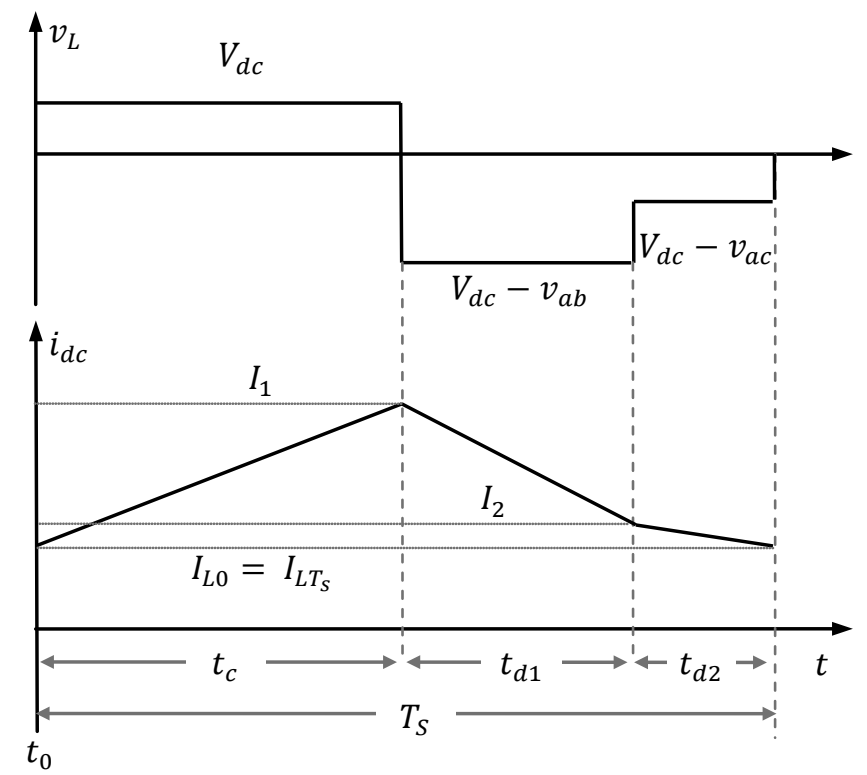

Figure 4.5: The voltage and current waveform of the dc-link inductor over one switching cycle in Sector (I)

$$
\begin{array}{ll}
I_{1}-I_{L 0}=\frac{V_{d c}}{L_{d c}} \cdot t_{c} & ; \text { for } t_{0}<t \leq t_{0}+t_{c} \\
I_{2}-I_{1}=\frac{V_{d c}-v_{a b}}{L_{d c}} \cdot t_{d 1} & ; \text { for } t_{0}+t_{c}<t \leq t_{0}+t_{c}+t_{d 1} \\
I_{L T_{s}}-I_{2}=\frac{V_{d c}-v_{a c}}{L_{d c}} \cdot t_{d 2} & ; \text { for } t_{0}+t_{c}+t_{d 1}<t \leq t_{0}+T_{s}
\end{array}
$$


where, $I_{L 0}$ and $I_{L T_{S}}$ are the dc-link inductor currents at the beginning and the end of one switching cycle, respectively. Also, $V_{d c}$ is the dc input voltage, $L_{d c}$ is the value of the inductor, $t_{c}$ is the time elapsed for charging the dc-link inductor, $t_{d 1}$ and $t_{d 2}$ are the timeinterval of injecting power from phase $a$ to phase $b$ and phase $c$ respectively, and $v_{a b}$ and $v_{a c}$ are the instantaneous line-to-line voltages. Adding up the above three equations yields:

$I_{L T_{S}}-I_{L 0}=\frac{V_{d c}}{L_{d c}} \cdot t_{c}+\frac{V_{d c}-v_{a b}}{L_{d c}} \cdot t_{d 1}+\frac{V_{d c}-v_{a c}}{L_{d c}} \cdot t_{d 2}$

Thus, one can write as follows:

$I_{L T_{s}}-I_{L 0}=\frac{V_{d c}}{L_{d c}} \cdot\left(t_{c}+t_{d 1}+t_{d 2}\right)-\left(\frac{v_{a b}}{L_{d c}} \cdot t_{d 1}+\frac{v_{a c}}{L_{d c}} \cdot t_{d 2}\right)$

For a fixed switching frequency, $T_{s}$ is constant and also $t_{c}+t_{d 1}+t_{d 2}=T_{s}$. Thus, (4.5) can be simplified as:

$I_{L T_{S}}-I_{L 0}=\frac{V_{d c}}{L_{d c}} \cdot T_{S}-\left(\frac{v_{a b}}{L_{d c}} \cdot t_{d 1}+\frac{v_{a c}}{L_{d c}} \cdot t_{d 2}\right)$

Substituting, $I_{L T_{S}}-I_{L 0}$, by $\Delta I_{L}$, one can write:

$V_{d c} \cdot T_{s}-\Delta I_{L} \cdot L_{d c}=v_{a b} \cdot t_{d 1}+v_{a c} \cdot t_{d 2}$

Notice, one can write the line-to-line reference voltages as follows:

$v_{a b}=\sqrt{3} V_{m} \cdot \cos (\omega t)$
$v_{b c}=\sqrt{3} V_{m} \cdot \cos \left(\omega t-\frac{2 \pi}{3}\right)$
$v_{c a}=\sqrt{3} V_{m} \cdot \cos \left(\omega t-\frac{4 \pi}{3}\right)$

Thus, $v_{a c}$ is given by:

$v_{a c}=\sqrt{3} V_{m} \cdot \cos \left(\omega t-\frac{\pi}{3}\right)$

Let us pick the discharging time-intervals, $t_{d 1}>0$ and $t_{d 2}>0$, as follows: 


$$
\begin{aligned}
& t_{d 1}=m T_{s} \cos (\alpha) \\
& t_{d 2}=m T_{s} \cos \left(\alpha-\frac{2 \pi}{3}\right) \quad ; \text { for } \quad m>0, \frac{\pi}{6}<\alpha<\frac{\pi}{2}
\end{aligned}
$$

where, the positive coefficient $m$ is called the modulation index. It will be seen later, that this coefficient can be used to regulate the amplitude of the output voltage of the SSBI. Accordingly, one can write the following expressions:

$$
\begin{aligned}
& v_{a b} \cdot t_{d 1}=\left(\frac{m T_{s} \sqrt{3} V_{m}}{2}\right)(\cos (\omega t-\alpha)+\cos (\omega t+\alpha)) \\
& v_{a c} \cdot t_{d 2}=\left(\frac{m T_{s} \sqrt{3} V_{m}}{2}\right)\left(\cos \left(\omega t-\alpha+\frac{\pi}{3}\right)-\cos (\omega t+\alpha)\right)
\end{aligned}
$$

Substituting $v_{a b} . t_{d 1}$ by (3.11) and $v_{a c} . t_{d 2}$ by (3.12) in (3.7) yields:

$$
V_{d c} \cdot T_{s}-\Delta I_{L} \cdot L_{d c}=(3 / 2) m T_{s} V_{m} \cos \left(\omega t-\alpha+\frac{\pi}{6}\right)
$$

In order to keep (4.13) always valid, the right side term of this equation should be a timeindependent expression. Accordingly, $\alpha$ must follow $\omega t$ at the same angular speed, i.e. $\alpha=\omega t-\alpha_{0}$. Since the discharging time-intervals, $t_{d 1}$ and $t_{d 2}$, must be always positive values, then for Sector (I):

$$
\frac{\pi}{6}<\omega t-\alpha_{0}<\frac{\pi}{2} \stackrel{0 \leq \omega t<\frac{\pi}{3}}{=} \alpha_{0}=-\frac{\pi}{6}
$$

Hence, (3.13) can be rewritten as:

$$
V_{d c} \cdot T_{s}-\Delta I_{L} \cdot L_{d c}=\left(\frac{3}{2}\right) m T_{s} V_{m}
$$

As a result,

$$
m=\frac{2\left(V_{d c} \cdot T_{S}-\Delta I_{L} \cdot L_{d c}\right)}{3 V_{m} T_{S}}=\frac{2}{3}\left(\frac{V_{d c}}{V_{m}}-\frac{\Delta I_{L} \cdot L_{d c}}{V_{m} T_{S}}\right)
$$

Therefore, if $m$ in (4.10) is substituted by (4.16), the discharging times, $t_{d 1}$ and $t_{d 2}$, can be calculated, in steady-state and balanced conditions (i.e. $I_{L T_{S}} \approx I_{L 0}$ ), from the following equations; 
$t_{d 1} \approx \frac{2 V_{d c} \cdot T_{S}}{3 V_{m}} \cdot \cos \left(\omega t-\alpha_{0}\right)$

$t_{d 2} \approx \frac{2 V_{d c} \cdot T_{s}}{3 V_{m}} \cdot \cos \left(\omega t-\alpha_{0}-\frac{2 \pi}{3}\right)$

Alternatively, (4.17) and (4.18) can be also rewritten as:

$t_{d 1}=\frac{2 V_{d c} \cdot T_{S}}{3 V_{m}} \cdot \sin \left(\frac{\pi}{3}-\omega t\right)$

$t_{d 2}=\frac{2 V_{d c} \cdot T_{s}}{3 V_{m}} \cdot \sin (\omega t)$

which are exactly identical to what one can obtain from the trigonometric identity illustrated in Figure 4.3, if $\left|V_{a b}\right|$ and $\left|V_{a c}\right|$ are replaced by $\sqrt{3} V_{m}$.

\subsubsection{Control Strategy}

The control strategy of inverters depending on their mode of operation, either stand-alone or grid-connected, can be essentially different. The operation goal of a gridconnected inverter is usually to inject some certain amounts of active and reactive power into the grid. However, on the other hand, a stand-alone inverter is intended to feed a local load with a certain (and usually fixed) voltage and frequency. Or more precisely, a three-phase stand-alone inverter has to produce balanced voltages at a given frequency. Looking at the previous section, one may observe that the proposed SSBI has two degrees of freedom, i.e. the angular displacement of the reference voltage phasor, $\omega t$, and the modulation index, $m$. While changing the phase angle of the reference angular displacement with respect to the angular displacement of the grid voltage causes a phase shift between the SSBI's output voltage and the grid voltage, changing the modulation 
index affects the amplitude of the output voltage. As can be seen in (4.16), $m$ is reciprocally related to the $V_{m}$.

Thus, it can be concluded that:

i) In the grid-connected operation, the active power could be controlled through adjusting the phase angle of the reference voltage with respect to the grid, and the reactive power could be control through regulation of the modulation index.

ii) For the stand-alone mode of operation, the amplitude of the load voltage can be controlled by regulating the modulation index, i.e. the higher $m$, the lower $V_{m}$.

\subsubsection{Simulation and Experimental Results}

In order to verify the proposed PWM switching pattern, a set of simulation and experimental results is presented here. The studies have been carried out for a laboratoryscaled SSBI which was built for verification, see Figure 4.6, and tested for a resistiveinductive (RL) and a rectifier load. The switching signals were generated by a CLP1104 dSPACE system which was linked to MATLAB Simulink, and the switching frequency was chosen to be $2.88 \mathrm{kHz}$ because of the limitations of dSPACE. The measurements in this experiment were performed using a LeCroy Waverunner 64XI oscilloscope with one CP031 current probe, one CP030 current probe, and one ADP305 differential voltage probe. The bandwidth of the oscilloscope is $600 \mathrm{MHz}$, while the bandwidths of the CP031, CP030, and the ADP305 are $100 \mathrm{MHz}, 50 \mathrm{MHz}$, and $100 \mathrm{MHz}$, respectively. 
Figure 4.6 shows a scene of the experimental setup and Table 4.2 gives some information on the SSBI circuit elements.

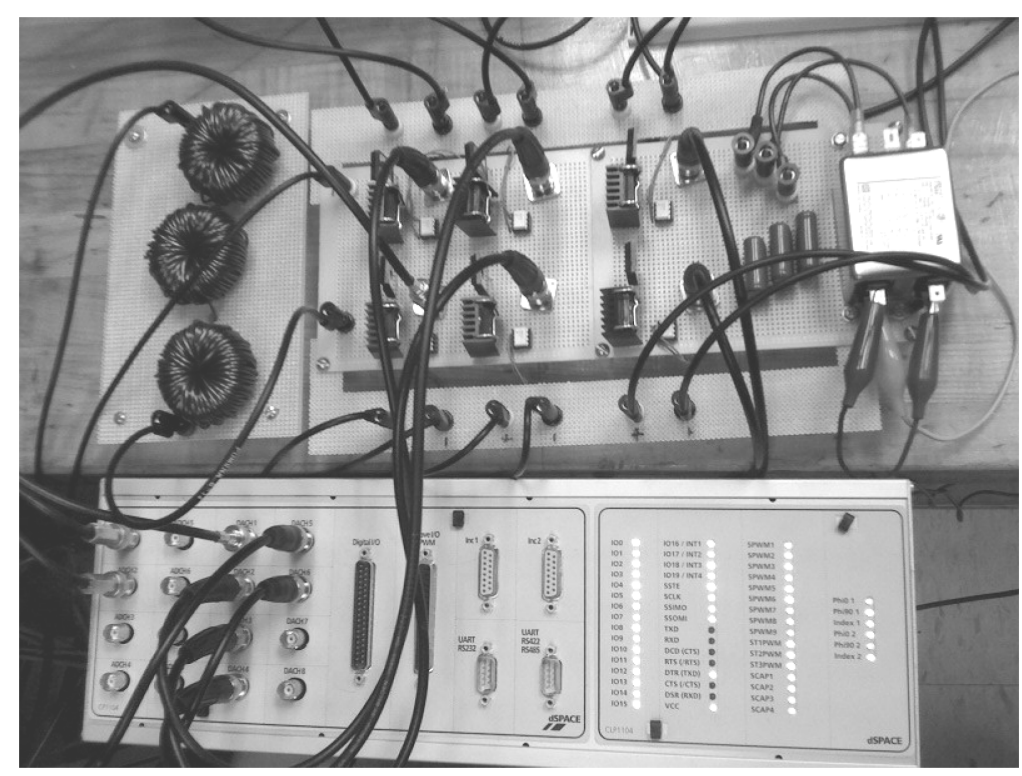

Figure 4.6: The experimental prototype

Table 4.2: Hardware specifications of the SSBI

\begin{tabular}{cc}
\hline Component & Specifications \\
\hline dc-source & XR125-32 Magna-Power Electronics \\
\hline MOSFETS & STx42N65M5 (TO-220) \\
\hline Diodes & IXGH 30 N60BDI (TO-247 AD) \\
\hline dc-Link Resistance, $R_{d c}$ & $0.1 \Omega$ \\
\hline dc-Link Inductance, $L_{d c}$ & $10 \mathrm{mH}$ \\
\hline ac-Side Capacitance, $C_{a c}$ & $10 \mu \mathrm{F}$ \\
\hline Line Frequency, $f_{1}$ & $60 \mathrm{~Hz}$ \\
\hline Switching Frequency, $f_{s}$ & $2.88 \mathrm{kHz}$ \\
\hline DSP Sampling Frequency, $f_{\text {samp }}$ & $87 \mathrm{kHz}$ \\
\hline
\end{tabular}

The experiments were performed for two different loads, i.e. a linear Y-connected RL load and a nonlinear full-bridge rectifier load. Since the rated power of a typical 
residential PV panel is less than a few hundred Watts [64], the operating point of the SSBI was selected for a terminal power about $150 \mathrm{~W}$. This could be achieved through choosing proper load parameters and adjustment of the modulation index. The operating points of the SSBI for the performed experiments are summarized in Table 4.3.

Table 4.3: Operating points of SSBI for nonlinear and linear loads

\begin{tabular}{ccc}
\hline Operating Point & Linear Load (RL Load) & Nonlinear Load (Rectifier Load) \\
\hline dc-Source Voltage $V_{d c}(\mathrm{~V})$ & 30 & 30 \\
\hline Modulation Index, $m$ & 0.41 & 0.41 \\
\hline Load Resistance, $R_{L}(\Omega)$ & 300 & 600 \\
\hline Load Inductance, $L_{L}(m H)$ & 26 & 0 \\
\hline
\end{tabular}
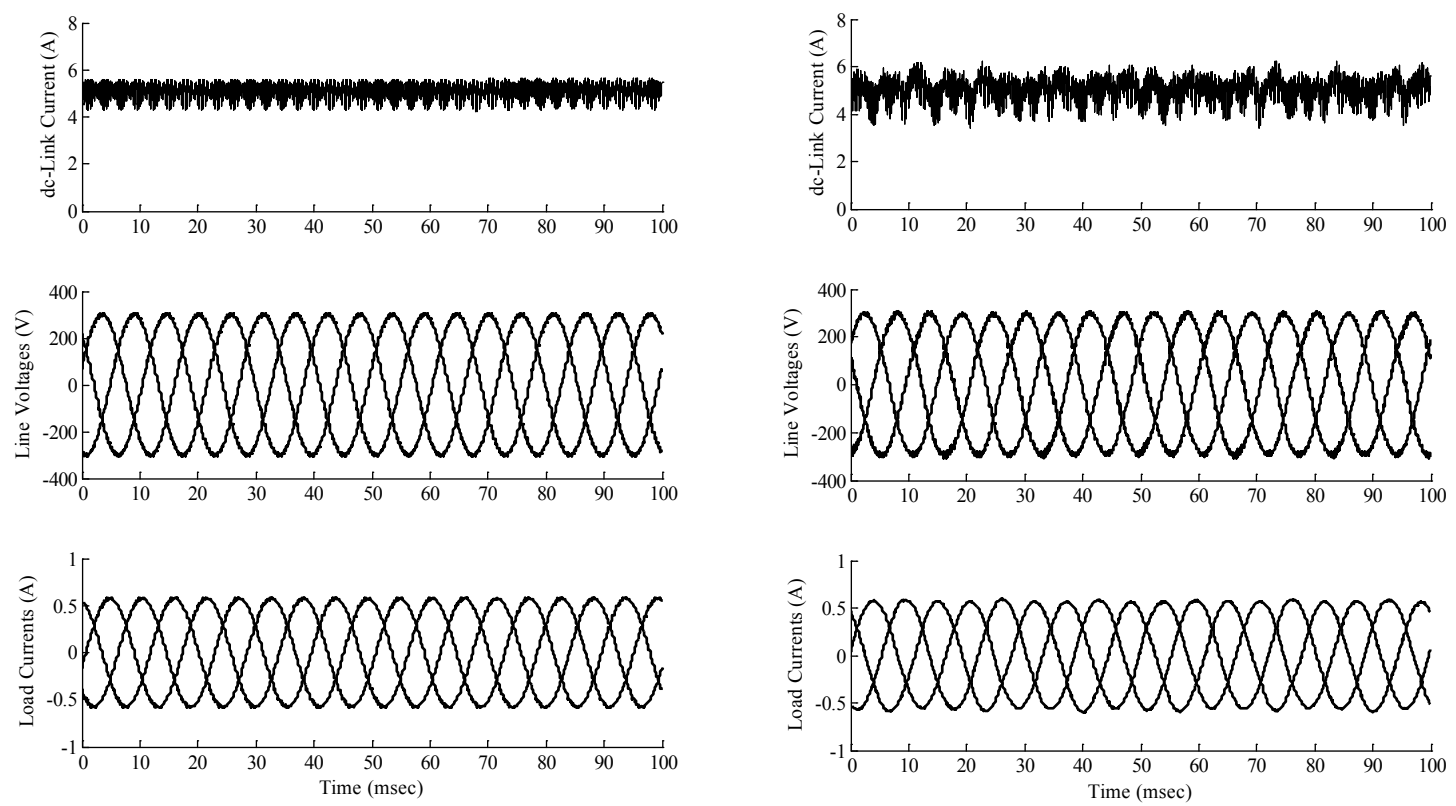

(a) $m=0.41$

(b) $m=0.40$

Figure 4.7: Waveforms of the SSBI for the linear RL load, a) simulation, b) experimental 
These operating points correspond to the maximum power point of the commercially available PV panel, BP 175 [65], which outputs $175 \mathrm{~W}$ at a voltage of 35.5 $V$. The output line voltage and current waveforms of simulation and experimental result, as well as the frequency spectrums of the experimental results are shown in Figures 4.710. As can be seen in these figures, the CBI topology in conjunction with the proposed switching pattern and control strategy is capable of feeding both linear and nonlinear loads with a decent quality.
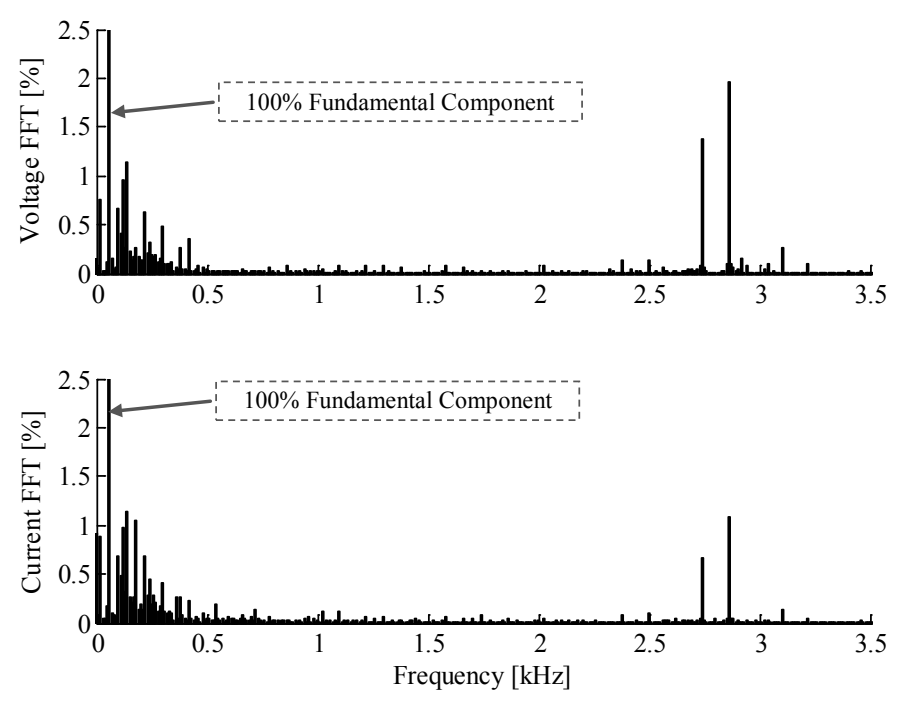

Figure 4.8: FFT Spectrum of the load terminal voltage and current for the linear RL load

The waveforms of the dc-link current, line-to-line load terminal voltages and current, for the linear RL load, are shown in Figure 4.7. As can be seen the SSBI can feed the load at ac line voltage of $208 \mathrm{~V}$, with a satisfactory quality. The corresponding voltage and current spectrums, for the experimental results, are demonstrated in Figure 4.8. The voltage and current THDs are $3.4 \%$ and $3.1 \%$, respectively. Also, the maximum 
non-fundamental harmonic components of the voltage and current are $1.96 \%$ and $1.1 \%$, respectively, which take place at the switching frequency, i.e. $2.88 \mathrm{kHz}$. The Standard IEEE-519 [66] recommends limiting the voltage THD to 5\% and the magnitude of each individual harmonic to $3 \%$. Comparing these limits with the obtained values indicates that the SSBI meets the required standards.
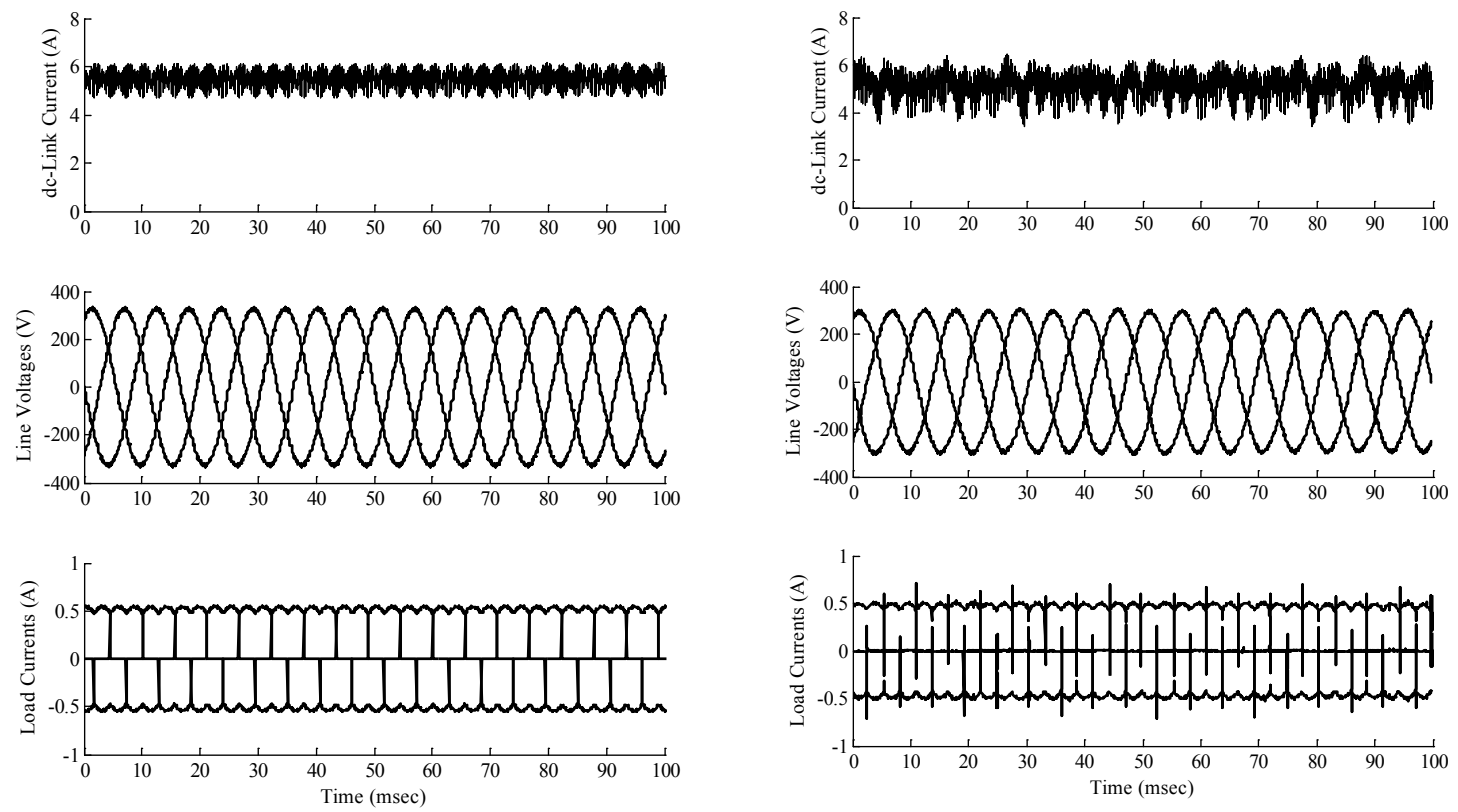

(a) $m=0.41$

(b) $m=0.40$

Figure 4.9: Waveforms of the SSBI for the nonlinear rectifier load, a) simulation, b) experimental

Similarly, the waveforms and frequency spectrums, for the nonlinear rectifier RL load, are shown in Figures 4.9-10. Again, it can be seen that SSBI can feed the load at a line voltage of $208 \mathrm{~V}$, with a satisfactory quality, although, because of the highly nonlinear nature of the rectifier load, the current quality is very poor. The voltage and current THDs are $3.5 \%$ and $31.1 \%$, respectively. Moreover, the maximum non- 
fundamental harmonic components of the voltage is $2.06 \%$, which takes place at the switching frequency, i.e. $2.88 \mathrm{kHz}$. However, in this case, the rectifier load draws large components of low-order harmonic currents from the inverter, i.e. $22.6 \%, 10.9 \%, 8.7 \%$, and $6 \%$, for 5 th, 7 th, 11 th, and 13 th order harmonics, respectively.
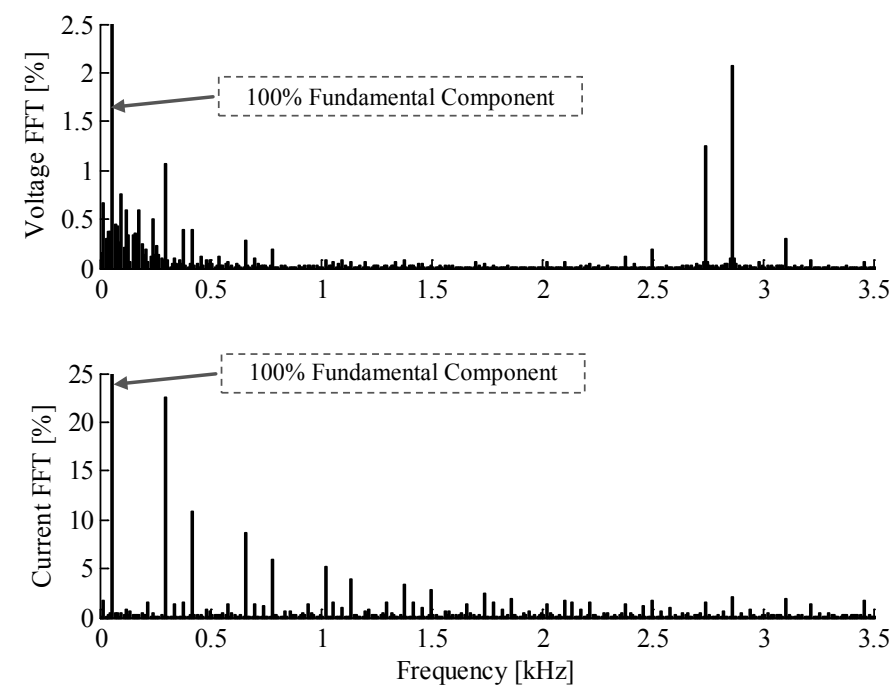

Figure 4.10: FFT Spectrum of the load terminal voltage and current for the nonlinear rectifier load

\subsubsection{Abnormal Conditions}

In this subsection, the operation of the three-phase SSBI under abnormal operating condition of short circuit is investigated and compared with a conventional sinusoidal pulse-width-modulated voltage source inverter (SPWM-VSI). The topology and switching scheme for the SPWM-VSI has been explained in Chapter 2. It is assumed that the SPWM-VSI, is supplied at $400 \mathrm{~V}$ from the dc-side, has an LC filter $\left(L_{f}=2 \mathrm{mH}\right.$ and $C_{f}=5 \mu F$ ) at the ac-side, and its modulation index is 0.84. Both SPWM-VSI and current source-based SSBI are initially supplying the RL load of Table 4.3 under normal 
conditions. At time, $t_{\text {fault }}=20 \mathrm{msec}$ a short circuit fault (with the fault resistance and inductance of $R_{\text {fault }}=0.1 \Omega$ and $L_{\text {fault }}=0.1 \mathrm{mH}$, respectively) occurs, which sustains for three cycles, i.e. $50 \mathrm{msec}$, and clears at $t_{\text {clear }}=70 \mathrm{msec}$. It should be noted that the fault currents (at the point of short circuit) cannot be interrupted until they cross zero, therefore, the fault currents may disappear a short while (no more than a half cycle) after the clearance time. Moreover, it is assumed that the neutral point of the Y-connected RL load is connected to the ground and the dc side of the circuit is isolated from the ground. The schematic diagram of the power conversion system, the load, and the short circuit fault is depicted in Figure 4.11. Performances of the inverters are compared for three major faults, i.e. line-to-ground (LG), line-to-line (LL), and three-phase (LLL) short circuits. The comparisons are made based on the obtained waveforms of the dc-link current, line-to-line voltages, and line currents, during and after the faults.

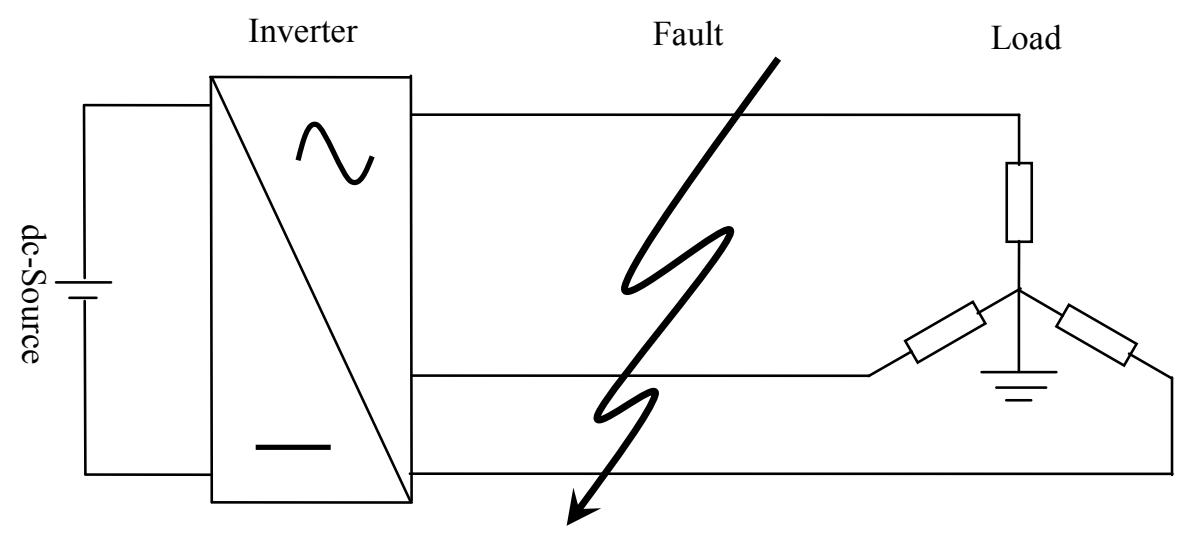

Figure 4.11: Short circuit fault on the dc-ac conversion systems 


\section{A. Line-to-Ground Short Circuit (LG Fault)}

The waveforms of the SPWM-VSI and SSBI under a line-to-ground fault are shown in Figure 4.12. As can be seen, this fault causes large $120 \mathrm{~Hz}$ ripples on the dc-link current of the SSBI, while it does not change the dc-link current of the SPWM-VSI significantly. The voltage waveforms indicate that the LG short circuit does not affect the line voltages of the SPWM-VSI, although the line-to-ground voltage of the faulty phase (phase $a$ ) becomes (almost) zero during the fault. On the other hand, the LG short circuit has noticeable impacts, like a drop in the line voltage amplitudes as well as distortions in their waveforms, on the line voltages of the SSBI. This means that, in term of the voltage profiles, the SPWM-VSI has a better performance than the SSBI under an LG short circuit fault.
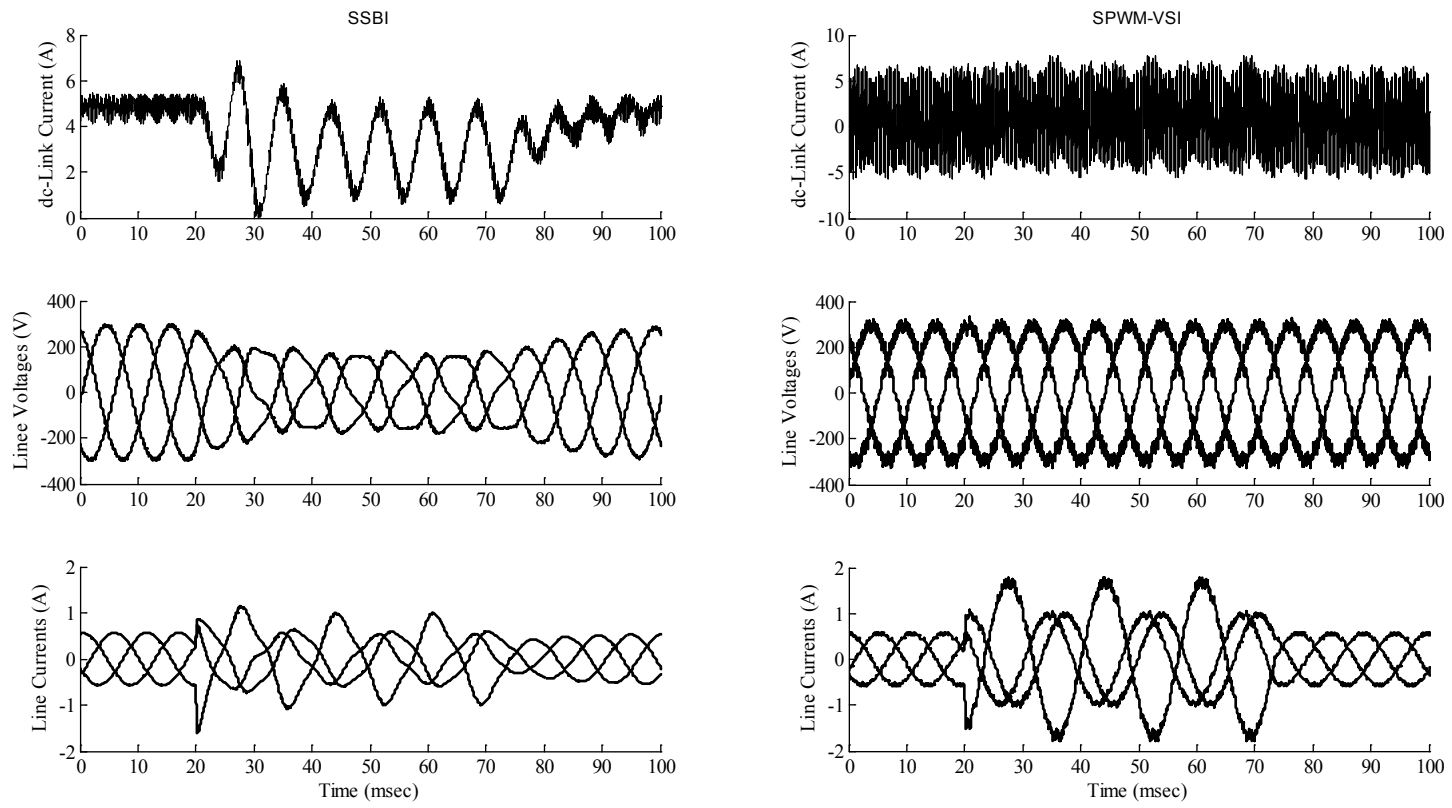

(a)

(b)

Figure 4.12: Performance of the inverters under an LG short circuit fault, a) SSBI, b) SPWM-VSI 
Short circuit level (SCL), or the peak current amplitude under short circuit, is the most critical measure of the short circuit faults. The reason is that the short circuit faults usually cause large currents flow in the circuit, which can result in serious damage to the system components. The obtained waveforms show that the SSBI has a peak current of $1.6 A$ (in fact $-1.6 A$ ), while this values is about $1.8 A$ for the SPWM-VSI. This means an $11 \%$ lower SCL for the SSBI than the SPWM-VSI.

\section{B. Line-to-Line Short Circuit (LL Fault)}

Figure 4.13 presents the waveforms of the SPWM-VSI and SSBI under a line-toline fault. It can be seen that the LL fault causes an up to $270 \mathrm{~A}$ current in the dc-link of the SPWM-VSI, which is significantly larger than that for the SSBI. It is also observed that while the voltage between the faulty phases (phases $a$ and $b$ ) is very close to zero, $v_{b c}$ and $v_{c a}$ have opposite angles ( $180^{\circ}$ phase difference). Obviously, this voltage profile is not suitable for supplying three-phase loads. Furthermore, the voltage of SPWM-VSI has some fluctuations after the fault clearance, which may arise from a resonance between the LC filter and the load.

In terms of short circuit currents, the SSBI considerably outperforms the SPWMVSI, i.e. the peaks of the transient and sustaining short circuit currents of the SSBI are 55 and $17 \mathrm{~A}$, respectively, whereas the SPWM-VSI has a peak current of $280 \mathrm{~A}$. 

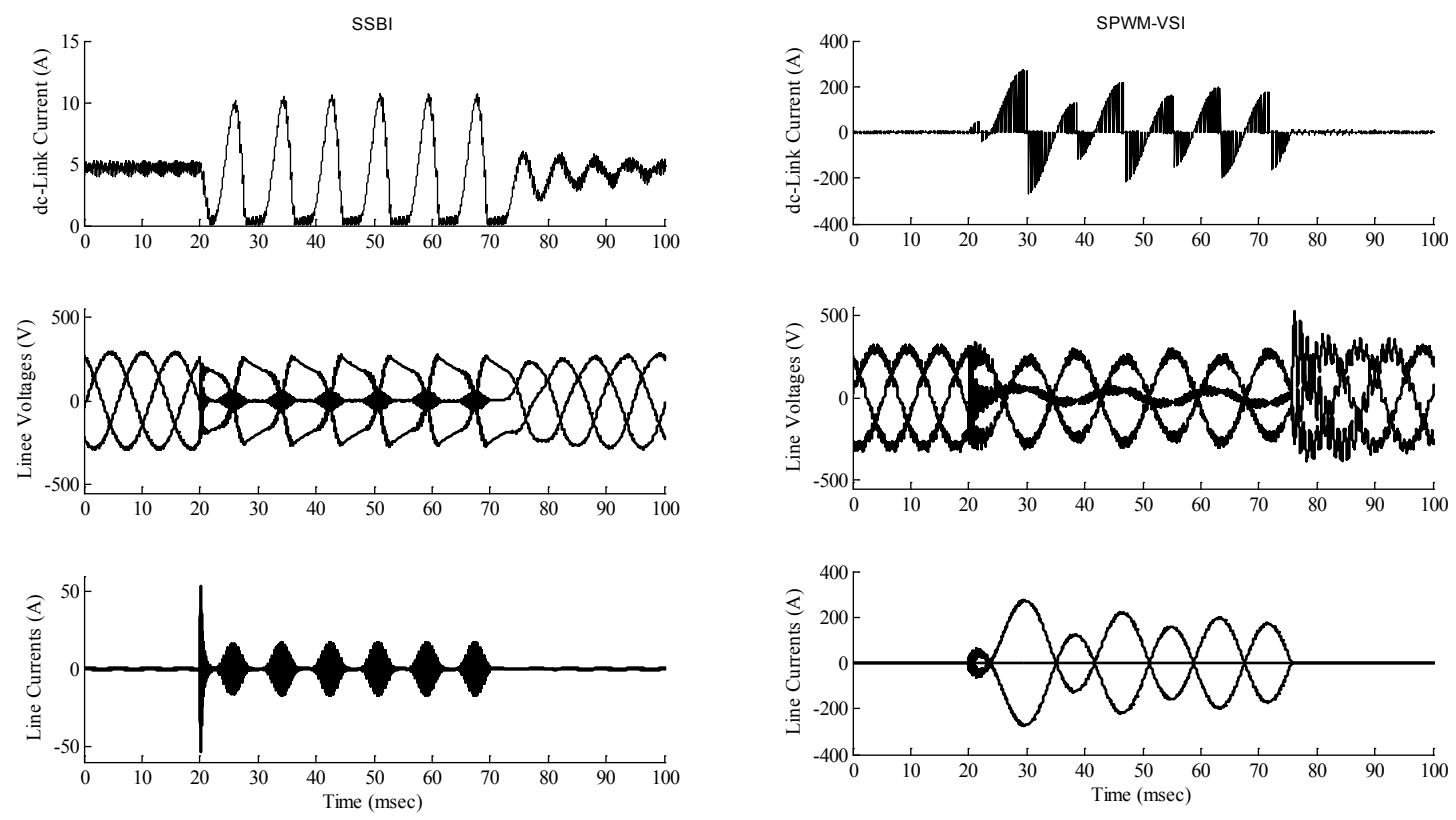

(a)

(b)

Figure 4.13: Performance of the inverters under an LL short circuit fault, a) SSBI, b) SPWM-VSI

\section{Three-Phase Short Circuit (LLL Fault)}

The waveforms of the SPWM-VSI and SSBI under a three-phase short circuit are shown in Figure 4.14. As can be observed in the figures, although the dc-link current of the SPWM-VSI goes extremely high (about $350 \mathrm{~A}$ ), the SSBI's current is limited to $11 \mathrm{~A}$ during the fault, and temporarily increases to $13 \mathrm{~A}$ after the fault clears. Moreover, both inverters have poor voltage profiles during the fault. However, the SSBI recovers faster after the fault clears.

It is evident from the obtained waveforms that there is a significant difference between the short circuit levels of the SSBI and SPWM-VSI. While the peak short circuit 
current of the SPWM-VSI is about $350 \mathrm{~A}$, the SSBI has a transient peak current of $75 \mathrm{~A}$, and a sustaining peak of $34 A$.
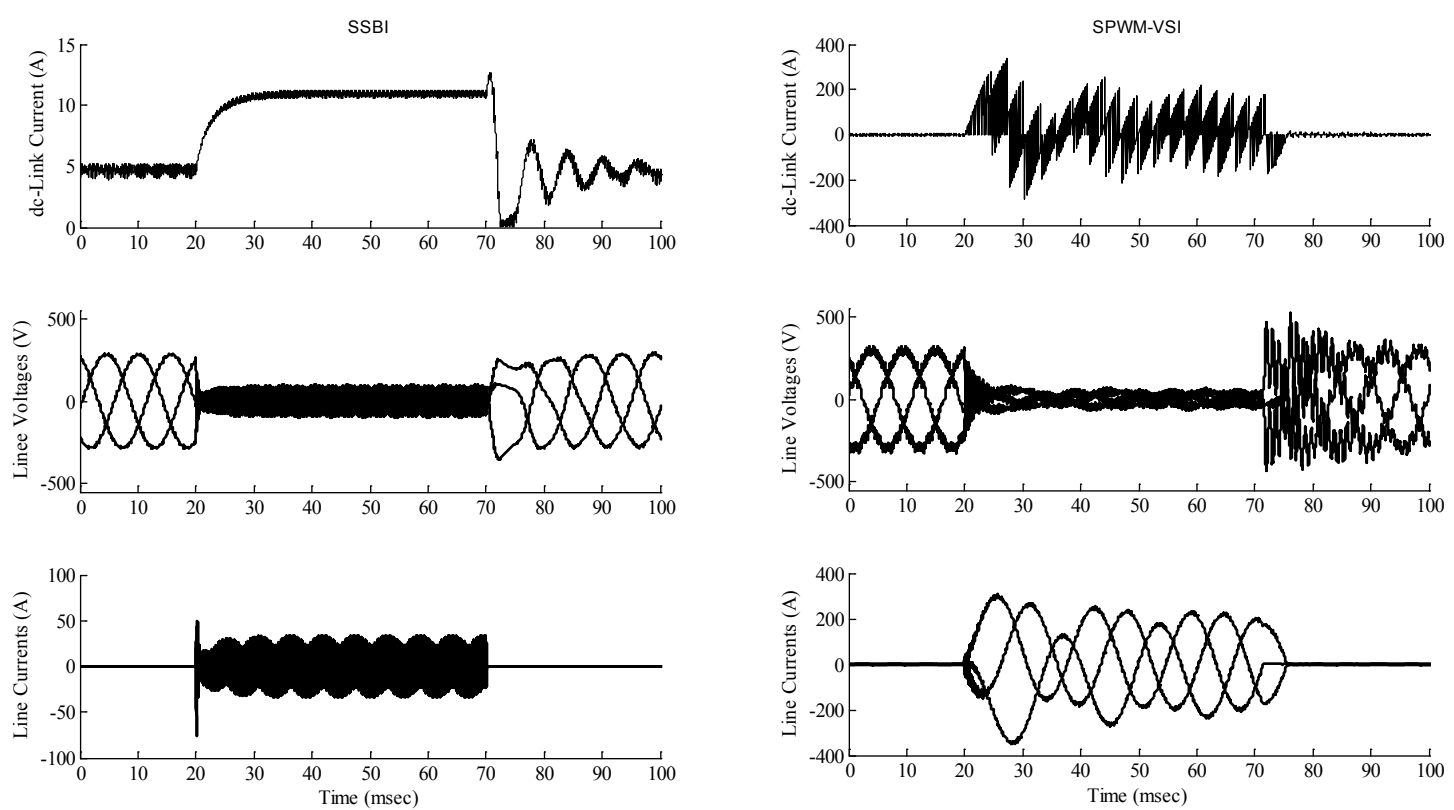

(a)

(b)

Figure 4.14: Performance of the inverters under an LLL short circuit fault, a) SSBI, b) SPWM-VSI

To conclude, the obtained results indicate that the SSBI has a superior performance to the SPWM-VSI under abnormal conditions of short circuit faults. It is also worth mentioning that, line-to-line-to-ground (LLG) is another major type of short circuit fault, which is usually investigated in short circuit studies. This fault has been also studied in this research work; however, since the obtained waveforms were quite similar to those for the LL fault, the results have not been presented here. 


\subsection{Single-Phase Single-Stage Boost Inverter}

The same concept of the three-phase SSBI can be applied to single-phase current source inverters in order to converter a low dc voltage to a high ac voltage in only one stage of conversion. This has been done in [5], where new switching pattern has been proposed for adapting the single-phase CSI to a single-phase SSBI. In the new switching pattern, only one switch operates at a high frequency rate, while two other switches conduct at any given instance. The high frequency switch is modulated with a variable rate proportional to a sinusoidal function, which should be in phase with the grid voltage to inject merely an active power.

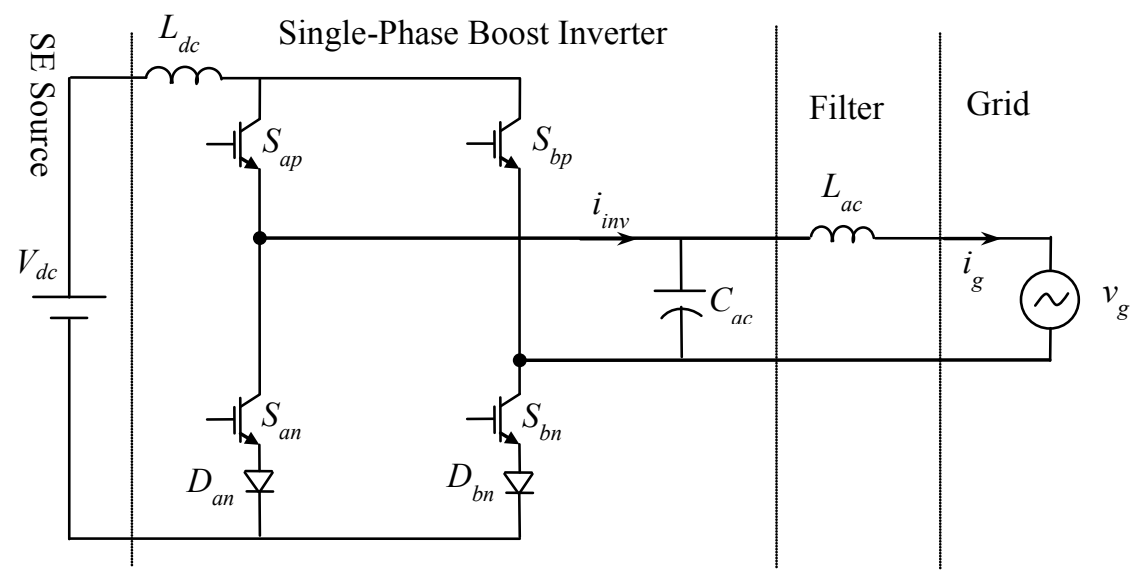

Figure 4.15: The complete power circuit schematic diagram of the CSI-based single-phase boost inverter

\subsubsection{Circuit Topology}

Figure 4.15 shows the complete power circuit schematic of the single-phase boost inverter, where $V_{d c}$ is a dc voltage source (representing an SE source), $L_{d c}$ is the dc-link inductor, $C_{a c}$ represents the ac-side film capacitor, and $L_{a c}$ represents the line inductor. 


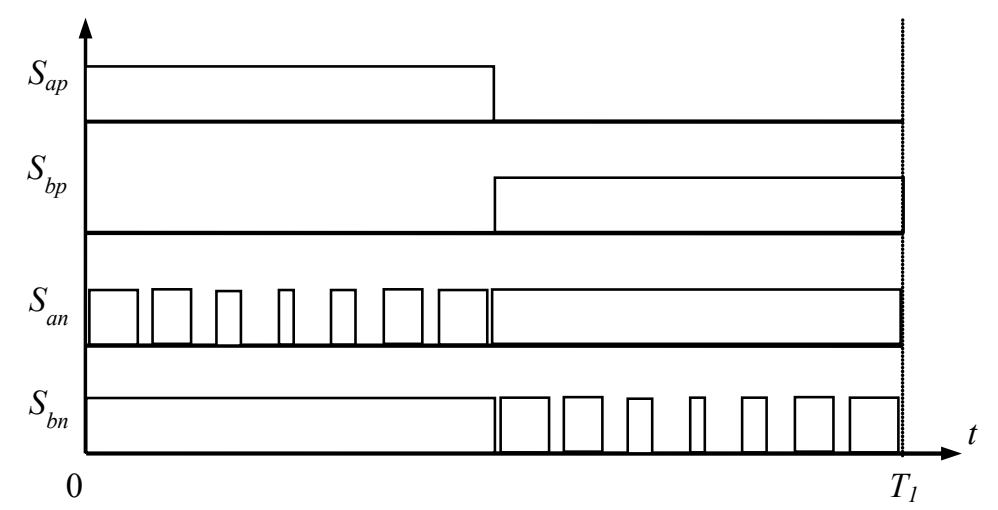

Figure 4.16: The switching pattern, one switch is modulated over each positive or negative half cycle in the single-phase SSBI
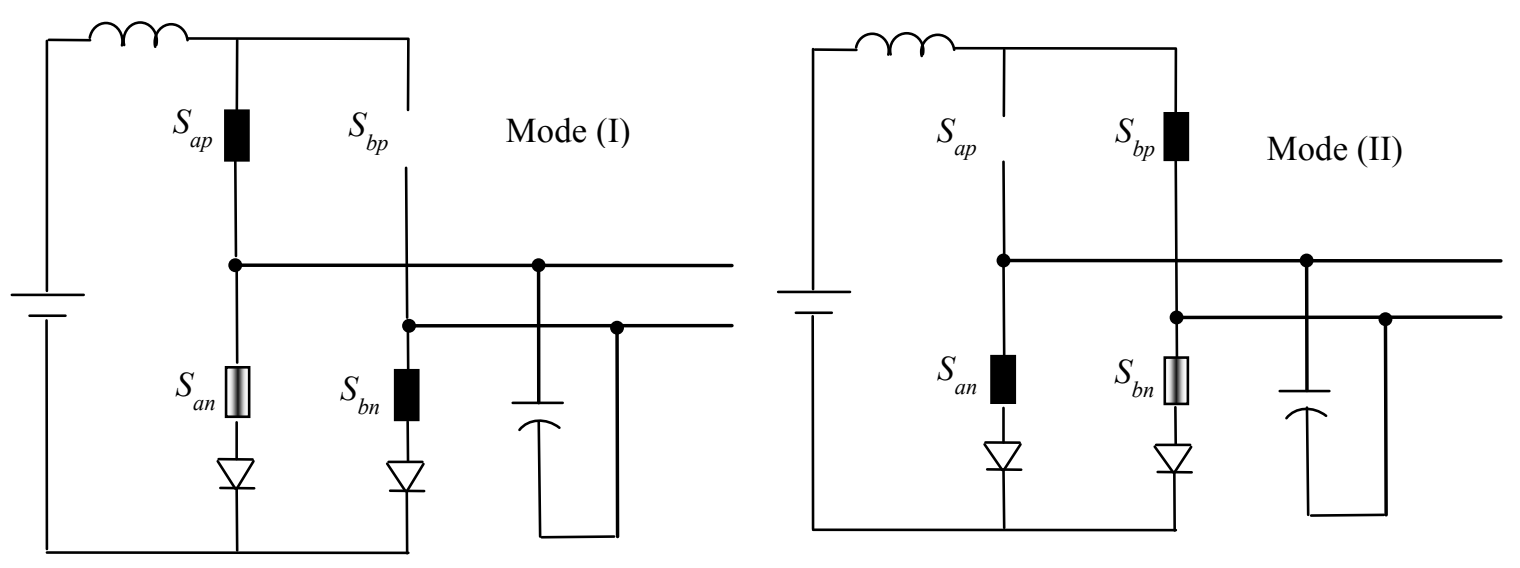

Figure 4.17: Switch positions, positive (Mode I) and negative (Mode II) half cycles

In this circuit topology, at any given instant, one of the upper switches and one of the lower switches conduct, while at any charging time-interval two switches of a leg are closed. During the charging times the magnetic energy in $L_{d c}$ is increased to boost the output voltage and inject power to the output circuit. The proposed switching pattern and, the associated operation of the switches during the positive and negative half cycles are 
shown in Figures 4.16-17. As can be seen in Figure 4.17, the boost inverter acts like a regular dc-dc boost converter in each of the modes.

\subsubsection{Switching Pattern}

In this section, the proposed switching pattern for the steady-state operation of the SSBI is presented. If the discharging time-interval, $0<t_{d}<T_{s}$, sinusoidally varies as:

$t_{d}(n)=m T_{s}\left|\sin \left(\omega_{1} t_{n}-\alpha_{0}\right)\right|$

The charging time-interval can be obtained as follows:

$t_{c}(n)=T_{s}-t_{d}(n)=T_{s}\left(1-m\left|\sin \left(\omega t_{n}-\alpha_{0}\right)\right|\right)$

where, $T_{s}$ is the switching cycle, $t_{n}=n T_{s}$ for $n=1,2,3, \ldots, N_{p}$ and $N_{p}=T_{1} / 2 T_{s}$ is equal to the number of pulses in half a cycle, $\omega$ is the frequency of the grid voltage which is assumed to be $v_{g}=\sqrt{2} V_{g}^{r m s} \sin (\omega t)$, and $0<m \leq 1$ and $\alpha_{0}$ are the modulation index and angle, respectively. It should be noted that, $m$ and $\alpha_{0}$ have impacts on the output current THD and injected power to the grid. During each charging interval, depending on the polarity of the grid voltage, one leg is shorted to make a current path for the dc-source through the dc-link inductor. On the other hand, during any discharging time-interval one upper and one lower switch from the different legs are on, so the inductor current can be discharged into the grid. The on/off durations of the switches during each switching cycle are shown in Table 4.4 .

Table 4.4: On/off time-intervals of the switches during each switching cycle

\begin{tabular}{ccccc}
\hline $\operatorname{sign}\left(v_{g}\right)$ & $S_{a p}$ & $S_{b p}$ & $S_{a n}$ & $S_{b n}$ \\
\hline+ & $T_{s}$ & 0 & $t_{c}$ & $t_{d}$ \\
\hline- & 0 & $T_{s}$ & $t_{d}$ & $t_{c}$ \\
\hline
\end{tabular}


As can be seen in this table, the switching state of each upper switch changes at the frequency of the grid voltage, $f_{1}=\omega / 2 \pi$, whereas the lower switches operate at the switching frequency, $f_{s}=1 / T_{s}$. In other words, in order to produce a single-stage boost dc-ac conversion, two upper switches must operate at the grid frequency and two lower switches must run at high frequency. However, by looking at the inverter topology, one may realize that it is not necessarily needed to turn both lower switches on and off on a complementary manner. Because of the fact that, during the charging intervals the lower series diodes, $D_{a n}$ and $D_{b n}$ in Figure 4.15 , block the reverse flow path for $i_{i n v}$ to circulate through $S_{a n}$ and $S_{b n}$ and the output capacitor, see Figure 4.17. Hence, it is alternatively possible to keep one of the lower switches, which is associated with the discharge interval, $t_{d}$, on during the entire switching cycle. The proposed on/off time-intervals (versus the initially-proposed in Table 4.4), is shown in Table 4.5.

Table 4.5: Proposed switching table during each switching cycle, $T_{S}$

\begin{tabular}{ccccc}
\hline $\operatorname{sign}\left(v_{g}\right)$ & $S_{a p}$ & $S_{b p}$ & $S_{a n}$ & $S_{b n}$ \\
\hline+ & $T_{s}$ & 0 & $t_{c}$ & $T_{s}$ \\
\hline- & 0 & $T_{s}$ & $T_{s}$ & $t_{c}$ \\
\hline
\end{tabular}

As can be seen, during each half cycle of the grid voltage, one switch operates at high frequency, $f_{s}$, and the other switches operate at the grid frequency, $f_{1}$. The proposed switching operation can best be demonstrated in Figure 4.16. As will be seen in the later sections, this modification leads to lower switching losses and, consequently, the higher inverter efficiency. 


\subsubsection{Control Strategy}

The control strategy of single-phase SSBI is like its three-phase counterpart, i.e. in the grid-connected mode, the operation goal is usually to inject active and reactive powers to the grid, while a stand-alone inverter is intended to feed a local load with a fixed voltage and frequency. Similar to that for the three-phase SSBI, in the gridconnected operation, the active power could be controlled through adjusting the phase angle of the reference voltage with respect to the grid, $\alpha_{0}$, and the reactive power could be control through regulation of the modulation index, $m$. On the other hand, for the stand-alone mode of operation, the amplitude of the load voltage can be controlled by regulating the modulation index.

\subsubsection{Simulation and Experimental Results}

In order to verify the proposed switching pattern, a set of simulation and experimental results is presented in this section. In this study, three different commercial PV panels, which are widely available in the market, are considered for the test purpose [65, 67-68]. The electrical specifications of these typical panels are summarized in Table 4.6.

Table 4.6: Electrical specifications of the PV panels

\begin{tabular}{cccc}
\hline Panel Model & BP 3110 & BP 4175B & BP 3230T \\
\hline$P_{M P P}(\mathrm{~W})$ & 110 & 175 & 230 \\
\hline$V_{M P P}(\mathrm{~V})$ & 16.9 & 35.4 & 29.1 \\
\hline$I_{M P P}(\mathrm{~A})$ & 6.50 & 4.94 & 7.90 \\
\hline$V_{O C}(\mathrm{~V})$ & 21.6 & 43.6 & 36.7 \\
\hline$I_{S C}(\mathrm{~A})$ & 7.40 & 5.45 & 8.40 \\
\hline
\end{tabular}



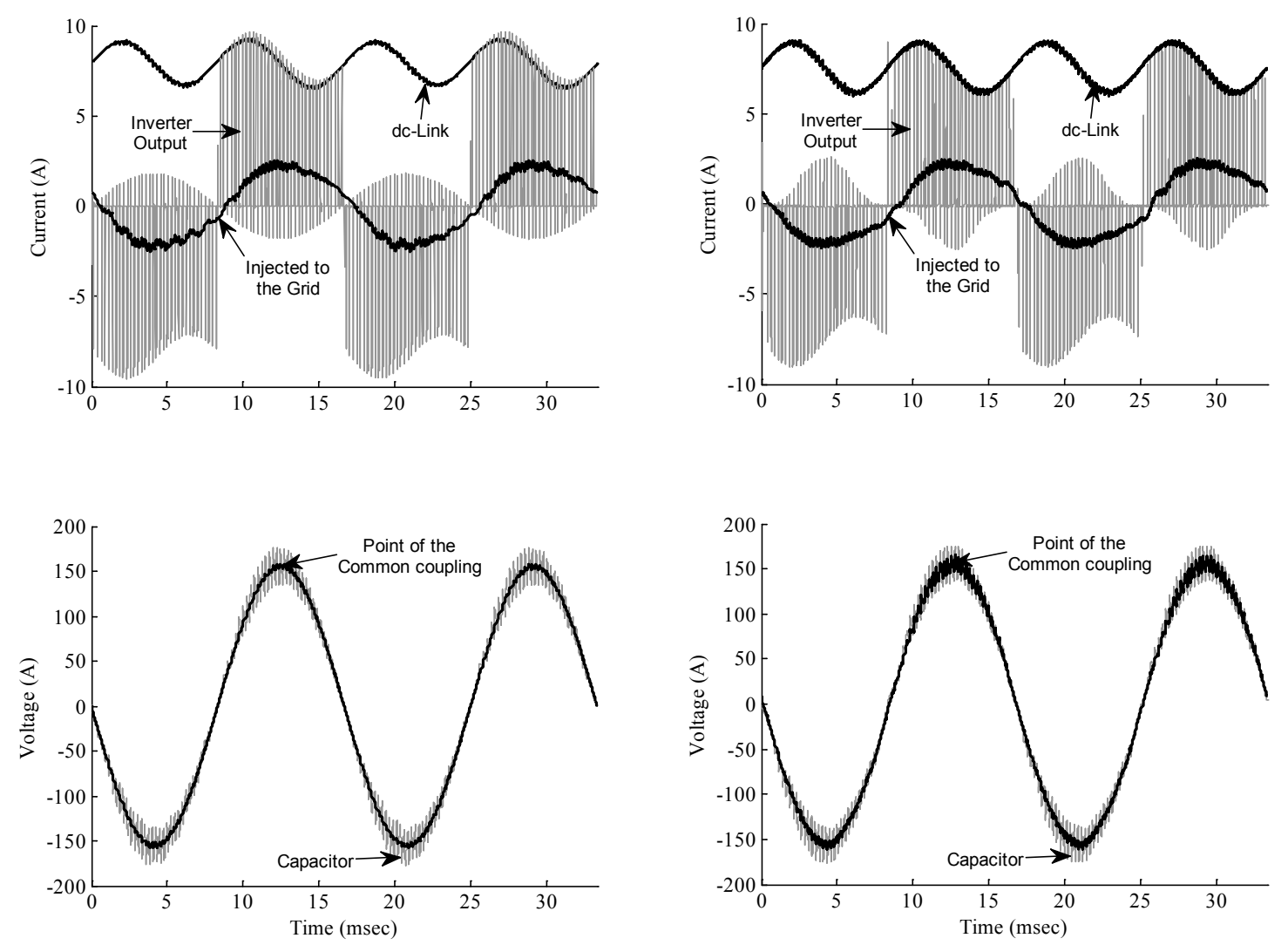
(a) $m=0.290, \alpha_{0}=0$
(b) $m=0.0308, \alpha_{0}=-6^{\circ}$

Figure 4.18: Performance of the single-stage boost inverter at the MPP of BP3230T, a) Simulation, b)

\section{Experimental}

The developed Simulink model has exactly the same topology as Figure 4.15. The circuit's parameters of the model, which are summarized in Table 4.7, are based on the actual values of the experimental setup that has been developed for testing the prototype single-phase SSBI. Here, $L_{g}$ and $R_{g}$ represent the inductance and resistance of the ac grid and the laboratory's autotransformer. The voltage at the point of common coupling is set to be $110 \mathrm{Vrms}$ at $60 \mathrm{~Hz}$. The MOSFET switches have a voltage drop of about $1 \mathrm{~V}$ and a 
static drain-source resistance, $R_{\text {on }}$, of $0.067 \Omega$, at a drain current of $5 \mathrm{~A}$. Also, the forward bias voltage of the diode is roughly $1 V$ for the same current. The inverter switching frequency is $f_{s}=4096 \mathrm{~Hz}$. Moreover, it is worth it to mention that, in order to suppress the voltage spikes across switches and diodes, appropriate snubber circuits have to be incorporated into the inverter circuit. In this work, the switches and diodes were equipped with RDC ( $R=35 \Omega$ and $C=10 \mathrm{nF})$ turn-off snubbers, and series RC ( $R=20 \Omega$ and $C=16 n F)$ snubbers, respectively.

Table 4.7: The circuit parameters of the simulation and hardware setup

\begin{tabular}{ccccccccc}
\hline$L_{d c}$ & $R_{d c}$ & $C_{a c}$ & $L_{f}$ & $R_{f}$ & $L_{g}$ & $R_{g}$ & MOSFET & DIODE \\
\hline $23.9 \mathrm{mH}$ & $0.3 \Omega$ & $10 \mu F$ & $2.55 \mathrm{mH}$ & $0.2 \Omega$ & $0.5 \mathrm{mH}$ & $1.4 \Omega$ & $\begin{array}{c}\text { STx42N65M5 } \\
\text { (TO-220FP) }\end{array}$ & APT40DQ120BG \\
\hline
\end{tabular}

The steady-state waveforms of the single-phase SSBI for $V_{d c}=29.1 \mathrm{~V}$ and $m=0.29$ are shown in Figure 4.18-(a). The average dc-link current under this condition is $I_{d c}=7.9 \mathrm{~A}$ that corresponds to the MPP of the $230 \mathrm{~W}$ PV panel, i.e. BP3230T. The $120 \mathrm{~Hz}$ ripple can be seen in the dc-link current, $i_{d c}$, as well as the inverter output current envelope. This will generate a set of low order harmonics in the injected current to the grid, $i_{g}$. This will be elaborated upon later in this chapter.

In order to verify the simulation results of the proposed PWM switching pattern, a laboratory scaled SSBI was built and tested while it was connected to the grid through the laboratory's transformer. The switching signals were generated by a CLP1104 dSPACE system, which was linked to MATLAB/Simulink, and the switching frequency was chosen to be $4096 \mathrm{~Hz}$. The measurements in this experiment were performed using a 
LeCroy Waverunner 64XI oscilloscope with two CP031 current probes, and two ADP305 differential voltage probes. The bandwidth of the oscilloscope is $600 \mathrm{MHz}$, while the bandwidths of the CP031 and the ADP305 are $50 \mathrm{MHz}$, and $100 \mathrm{MHz}$, respectively.

The steady-state waveforms of the single-phase SSBI, using the modifiedproposed switching pattern, for $v_{d c}=29.1 \mathrm{~V}$ and $m=0.308$, are shown in Figure 4.14(b). One can easily observe that the obtained graphs are in a remarkable agreement with those from the simulations. This can confirm the validity of the proposed methodology. Obviously, there are some minor differences between the simulation and the experimental results that are practically inevitable. These differences may arise from non-idealities of either the linear elements of the circuits like the inductors, transformer, and the capacitor, or the semiconductor devices such as the diodes and the MOSFETs. Furthermore, the inherent time delays of the measurement and control systems can also aggravate the differences. For instance, the voltage sensor and the control system have about $40 \mu \mathrm{sec}$ and $1 / f_{s}=244 \mu \mathrm{sec}$ delays, respectively. In order to compensate these delays, the reference discharging time-interval, $t_{d}$, should lead the line voltage by about $284 \mu \mathrm{sec}$. This can be applied through a 6 -degree shift, i.e. $\alpha_{0}=-6^{\circ}$. Moreover, as a consequence of the imperfect switching, resulting from the rise-time and fall-time delays of the semiconductor devises, as well as the delays associated with the isolation and drive circuits, the modulation indices can be slightly different in the simulations and experiments. It should be noted that ideal switches have been used in the simulation studies. 


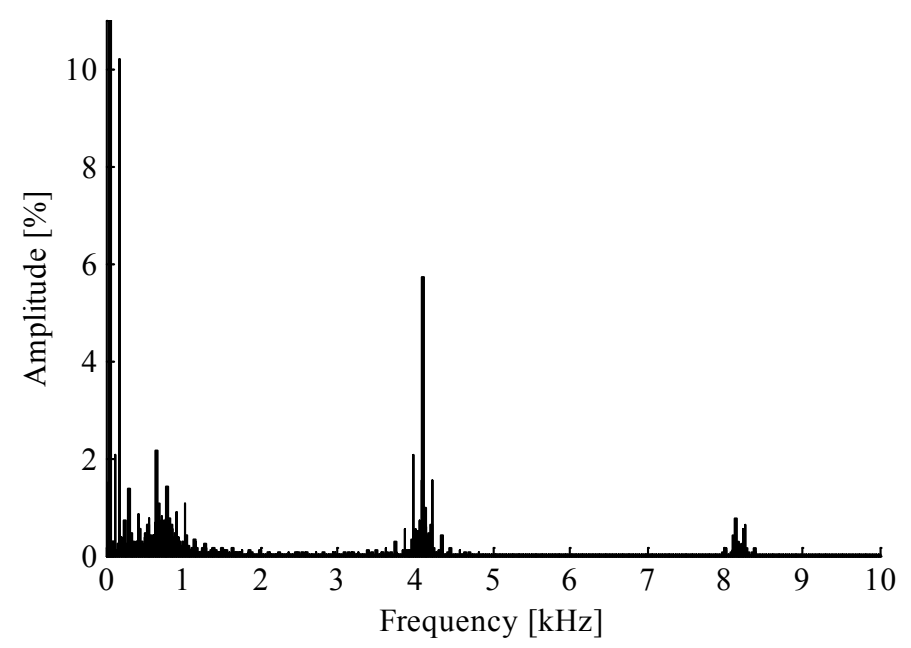

Figure 4.19: Harmonic contents of the injected current to the grid

Harmonic analysis of the injected current to the grid shows that the second and third harmonics are the major components of the total harmonic distortion. For instance, the harmonic content of $i_{g}$ for Figure 4.18-(b) is shown in Figure 4.19. As can be seen, the second and the third harmonics are $2.06 \%$ and $10.21 \%$, respectively (THD=13.47\%). Obviously the third harmonic has a significant contribution in the total harmonic distortion of the injected current. The rest of the grid current THD arises from the high order harmonics which are mostly at the switching frequency and its multiples (4.096 and $8.192 \mathrm{kHz}$ in Figure 4.19). Furthermore, one may observe some harmonics within the range of 700 to $1000 \mathrm{~Hz}$. These harmonics correspond to the natural frequencies of the circuit that will be discussed later in this section. In general, the ratio of the low order harmonics is proportional to the ratio between the ripple and the average of the dc-link current, i.e. the higher ripple to average, the more low harmonics. 
Besides the harmonic analysis, the characteristics of the presented single-phase SSBI, using the proposed switching pattern, are investigated here. This consists of the overall efficiency (including the output filter) and the grid current THD of the boost inverter versus the modulation index, $m$. It should be mentioned that deviations of the modulation angle from zero (in theory $\alpha_{0}=0$ and in practice $\alpha_{0}=-6^{\circ}$ ) can distort the injected current that significantly increases the current THD. The distortions appear as fluctuations around the natural frequencies of the circuit, i.e. within the range of 700 to $1000 \mathrm{kHz}$. Since the circuit shown in Figure 4.15 has three reactive elements, one may conclude that the single-phase SSBI has to have three natural frequencies. In order to reduce the complexities involved in calculating the natural frequencies of the actual inverter system, the circuit elements can be assumed ideal and the losses can be neglected. Then, for the charging interval:

$f_{1}=0$ and $f_{2,3}^{c}=\frac{ \pm 1}{2 \pi \sqrt{\left(L_{f}+L_{g}\right) C_{a c}}}= \pm 911.32 \mathrm{~Hz}$

and for the discharging interval:

$f_{1}=0$ and $f_{2,3}^{d}=\frac{ \pm 1}{2 \pi} \sqrt{\frac{L_{f}+L_{g}+L_{d c}}{\left(L_{f}+L_{g}\right) L_{d c} C_{a c}}}= \pm 967.72 \mathrm{~Hz}$

Obviously, $f_{1}=0$ does not affect the injected current to the grid. However, considering the simplifying assumptions, the absolute values of $f_{2,3}^{c}$ and $f_{2,3}^{d}$ are within the range of the observed oscillations. As was mentioned earlier in this section, these harmonic components can also be observed when the inverter output current is in phase with the grid voltage $\left(\alpha_{0}=0\right.$ or $\alpha_{0}=-6^{\circ}$ in practice). However, under this condition, they constitute a small portion of the grid current THD, see Figure 4.19. It should be also 
noted that all resistances in the circuit have been neglected in (4.23) and (4.24), and all switches and diodes have been assumed ideal. In spite of the simplifying assumptions, (4.23) and (4.24) give good approximations of the natural frequencies of the system. Based on the above discussion, the modulation angle will be fixed at zero in the rest of this chapter. The studies have been carried out for three different dc-source voltages, 16.9, 29.1, and $35.4 \mathrm{~V}$, which correspond to the MPP voltages of the case study PV panels in Table 4.6 .

The inverter input power, $P_{d c}$, and injected power to the grid, $P_{g}$, versus the modulation index, $m$, are plotted for three PV voltage levels in Figure 4.20. This figure demonstrates that increasing the modulation index, generally results in decreases in both powers, i.e. the larger modulation index the less dc-source power and injected power to the grid. As can be seen in the upper graph, for $v_{d c}=29.1 \mathrm{~V}$, the input power is $230 \mathrm{~W}$ while the modulation index is slightly above $0.30(m=0.308$ in Figure $4.14-(b))$. The injected power to the grid for this modulation index is about $180 \mathrm{~W}$, which corresponds to an efficiency of $78 \%$. Similarly, for the input voltages of 16.9 and $35.4 \mathrm{~V}$, the power becomes 110 and $175 \mathrm{~W}$ at the modulation indices around 0.17 and 0.41 , respectively. These operating points correspond to the injected powers of 78 and $146 \mathrm{~W}$, i.e. $71 \%$ and $83 \%$ efficiencies, respectively. 

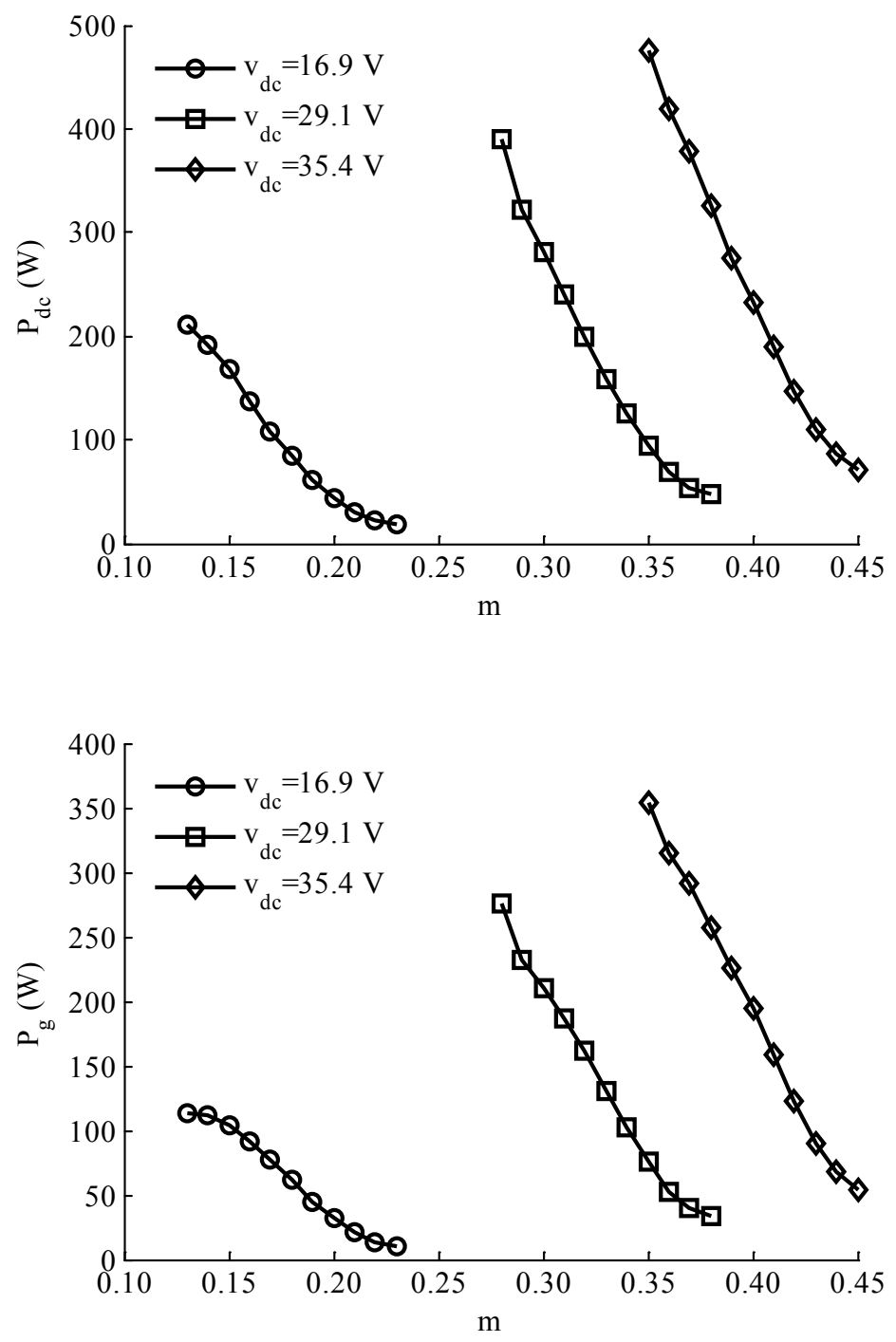

Figure 4.20: Variations of the dc-source power and the injected power to the grid versus the modulation index

The overall efficiency and the grid current THD of the proposed single-phase SSBI versus the injected power to the grid are shown in Figure 4.21. As can be seen in this figure, a larger input voltage in general results in a higher efficiency and a lower THD. Also, the grid current THD decreases with increasing the inverter output power, 
with a kind of exponential trend. However, unlike the THD, the efficiency does not monotonically change with the injected power. The upper graph indicates that the overall efficiency of the system, including the losses of the inverter and the snubbers, as well as the dc-link inductor and the ac filter, becomes maximum at some certain operating point. The maximum efficiency for the input voltages of 16.9, 29.1, and $35.4 \mathrm{~V}$ is achieved around the injected powers of 62,100 , and $160 \mathrm{~W}$, which result in $73.4 \%, 81.7 \%$, and 83.7\% efficiencies, respectively. Generally, in a photovoltaic energy conversion system, it is desired that the maximum efficiency of the conversion system (i.e. the inverter and the filter) coincides with the MMP of the PV panel. A comparison between the maximum achievable efficiencies $(73.4 \%, 81.7 \%, 83.7 \%)$ and the efficiency of the system at MMP of the panels $(71 \%, 78 \%$, and $83 \%)$, indicates that the prototype single-phase SSBI operates adequately close to its maximum efficiency at the MPP of the panels. This fact supports the optimal design of the prototype system.

In order to compare the performances of the two switching strategies mentioned in Table 4.4 and 4.5, (namely IPSP and MPSP, respectively) the experiments have been conducted for both cases. Also, in order to examine the impact of the switching frequency on the performance of the system, in addition to the original switching frequency $\left(f_{s}=4096 \mathrm{~Hz}\right)$, the MPSP strategy has been also applied for the switching frequency of $3072 \mathrm{~Hz}$. The overall efficiency (including the, dc-link inductor, inverter, and ac filter) and the current THD of the boost inverter, for different values of the modulation index, are shown in Figure 4.22. 

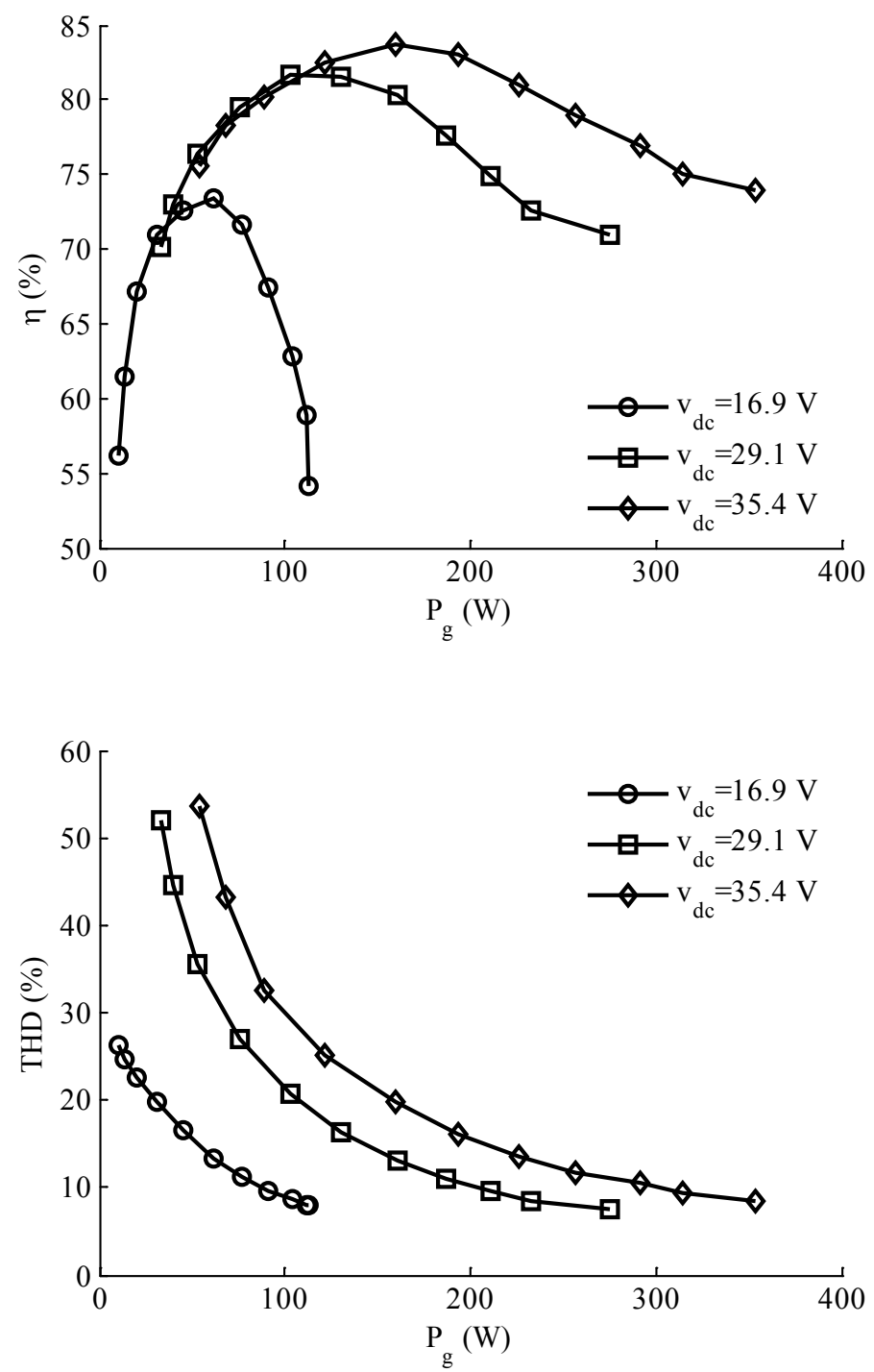

Figure 4.21: Variations of the efficiency and the THD of the single-stage boost inverter versus its injected power to the grid

As could be anticipated, in the case of MPSP, the system losses are less than the IPSP case. The reason is that in the MPSP only one switch operates at high frequency, whereas in the IPSP two switches operate at the high frequency. This results in 1-3\% improvement in the overall efficiency of the single-stage boost inverter. Furthermore, it is 
evident that reducing the switching frequency outperforms the efficiency of the system. As can be seen in this graph, the overall efficiency of the system for the switching frequency of $3.072 \mathrm{kHz}$ is about $1-2 \%$ more than that for the $4.096 \mathrm{kHz}$. Besides the efficiency considerations, the lower graph of Figure 4.22 indicates that the injected current to the grid in the MPSP case has a lower harmonic content than in the IPSP case. Quantitatively, employing the MPSP strategy enhances the grid current THD by 2-3\%. Moreover, as could be expected, increasing the switching frequency results in a higher current THD. The performance of the MPSP-operated single-phase single-stage boost inverter at the maximum efficiency and minimum THD, for different PV panels are summarized in Table 4.8.

Table 4.8: Performance of the single-phase SSBI for different PV panels at maximum efficiency and minimum THD

\begin{tabular}{|c|c|c|c|}
\hline Panel Model & Operating Parameters & Maximum Efficiency & Minimum THD \\
\hline \multirow{5}{*}{ BP3110 } & $m$ & 0.18 & 0.13 \\
\hline & $\eta(\%)$ & 73.4 & 54.1 \\
\hline & THD (\%) & 13.4 & 8.0 \\
\hline & $P_{g}(\mathrm{~W})$ & 62.0 & 112.9 \\
\hline & $r=V_{g}^{r m s} / V_{d c}$ & $110 / 16.9=6.51$ & $110 / 16.9=6.51$ \\
\hline \multirow{5}{*}{ BP4175B } & $m$ & 0.41 & 0.35 \\
\hline & $\eta(\%)$ & 83.7 & 73.9 \\
\hline & THD (\%) & 19.8 & 8.4 \\
\hline & $P_{g}(\mathrm{~W})$ & 159.2 & 353.4 \\
\hline & $r=V_{g}^{r m s} / V_{d c}$ & $110 / 3.54=3.12$ & $110 / 3.54=3.12$ \\
\hline \multirow{5}{*}{ BP3230T } & $m$ & 0.34 & 0.28 \\
\hline & $\eta(\%)$ & 81.7 & 70.9 \\
\hline & THD (\%) & 20.8 & 7.5 \\
\hline & $P_{g}(\mathrm{~W})$ & 102.8 & 275.6 \\
\hline & $r=V_{g}^{r m s} / V_{d c}$ & $110 / 29.1=3.78$ & $110 / 29.1=3.78$ \\
\hline
\end{tabular}



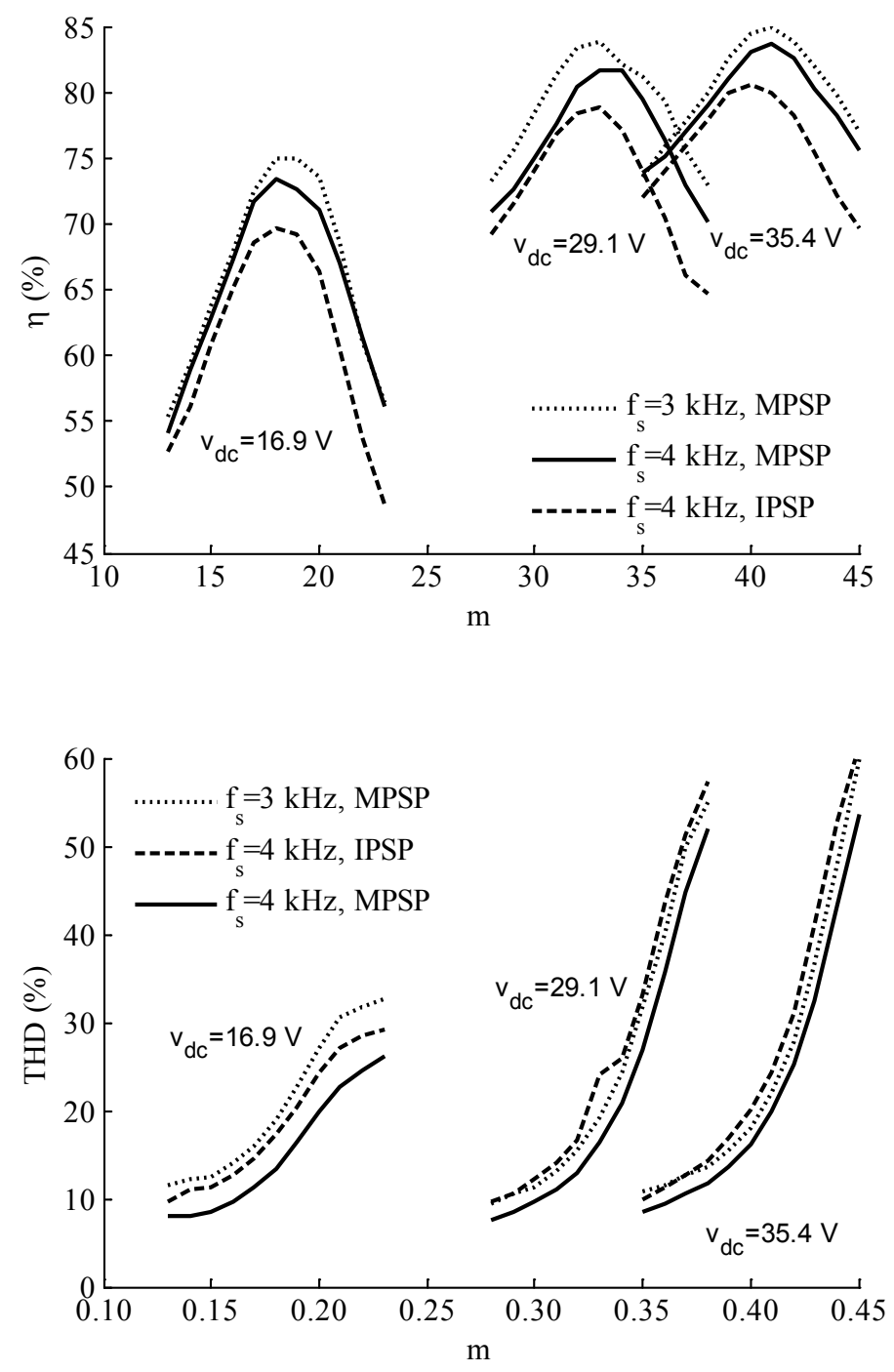

Figure 4.22: Variations of the overall efficiency and the THD of the single-stage boost inverter versus the modulation index

\subsection{Summary}

The idea of the recently proposed single- and three-phase single-stage boost inverters has been presented, and their fundamental equations have been derived. The 
circuit topologies, switching patterns, control strategies, and steady-state performance of the single- and three-phase SSBIs, have been explained in details and the concept has been proved through simulation and experiments.

The proposed switching pattern for the three-phase SSBI is based on the SVPWM concept; however, it is different form SVPWM. The presented switching pattern contains a total of nine switching states, including three charging states and six discharging states with only two switches conducting at any given instant. It has been demonstrated that the CSI topology in conjunction with the developed switching pattern is capable of providing the residential ac voltage from a low dc voltage of a single PV panel at its rated power for both linear and nonlinear loads. Besides that, the abnormal operation of the SSBI, under short circuit fault conditions, was studied. The obtained results indicated that the SSBI has a superior performance to its conventional counterpart, the SPWM-VSI.

The presented switching pattern for the single-phase SSBI contains a charging state and a discharging state over each switching cycle where only one switch operates at high frequency, one does not conduct, and the other two switches conduct for the entire switching cycle. In this method the charging state is necessary in order to boost the output voltage of PV arrays or fuel-cells.

In the next chapter the state-space averaging method will be applied to the standalone three-phase SSBI and its large- and small-signal models will be derived. 


\section{CHAPTER 5}

\section{STATE-SPACE-AVERAGED MODEL OF THE STAND-ALONE SINGLE- STAGE BOOST INVERTER SYSTEM}

The state-space-averaged model of the stand-alone single-stage boost inverter is developed and used for stability analysis and control design of the system. This is achieved through several steps; in the first step, the possible switching states of the circuit operation are enumerated and the associated state-space equations are derived and averaged over one switching cycle. Then, the obtained time-varying averaged model will be transformed into a synchronous $d q$-frame of reference in order to derive the timeinvariant model of the system. The system stability is investigated by eigenvalue sensitivity analysis and the findings will be verified through simulation and experiment.

This chapter contains five sections. The states of the system are enumerated in Section 1. The state-space-averaged model of the system is derived in Section 2. The eigenvalue sensitivity analysis of the system is performed in Section 3. The simulation

and experimental results are presented in Section 4, and Section 5 is the summary of the chapter.

\subsection{State Enumeration of the System}

The circuit diagram of a stand-alone three-phase single-stage boost inverter (SSBI) is presented in Figure 5.1. As was already discussed in the previous chapter, a three-phase SSBI operates in six sectors (see Figure 4.2), where each sector includes three switching states, i.e. one charging, and two discharging time-intervals. As can be 
seen in Figure 4.4, these switching states include a total of nine linear circuits, in which the three charging states have the same electrical equation, and the other six discharging circuits correspond to different circuit representations. Therefore, the operation of the SSBI is associated with seven distinct linear electric circuits, in which each circuit can be represented by a set of state-space equations. Therefore, the SSBI has seven state-space equations in total, and three state-space equations over each switching cycle. As a result, the SSBI is a linear time-varying system that, in general, can be represented in the following state-space form:

$\dot{\boldsymbol{x}}=\boldsymbol{A}(t) \boldsymbol{x}+\boldsymbol{B}(t) \boldsymbol{u}$

$\boldsymbol{y}=\boldsymbol{C}(t) \boldsymbol{x}+\boldsymbol{D}(t) \boldsymbol{u}$

where, for a $n$th order dynamical system with $r$ inputs and $m$ outputs: $\boldsymbol{x}$ is a $n \times 1$ vector (state vector), $\boldsymbol{u}$ is a $r \times 1$ vector (input vector), $\boldsymbol{y}$ is a $m \times 1$ vector (output vector), $\boldsymbol{A}$ is a $n \times n$ matrix (system matrix), $\boldsymbol{B}$ is a $n \times r$ matrix (control matrix), $\boldsymbol{C}$ is a $m \times n$ matrix (output matrix) and $\boldsymbol{D}$ is a $m \times r$ matrix [69]. The seven circuit diagrams, along with the corresponding system matrices for the stand-alone three-phase SSBI feeding a resistive load, $R_{L}$, (3rd order dynamical system) are presented in Table 5.1, where, $R_{d c}$ is the series resistance of the dc-link inductor. Just as an observation, it is interesting to mention that all discharging states, i.e. $D 1-D 6$, have the same eigenvalues, which means that they are all similar matrices [69]. 
Table 5.1: The possible states of the stand-alone SSBI system

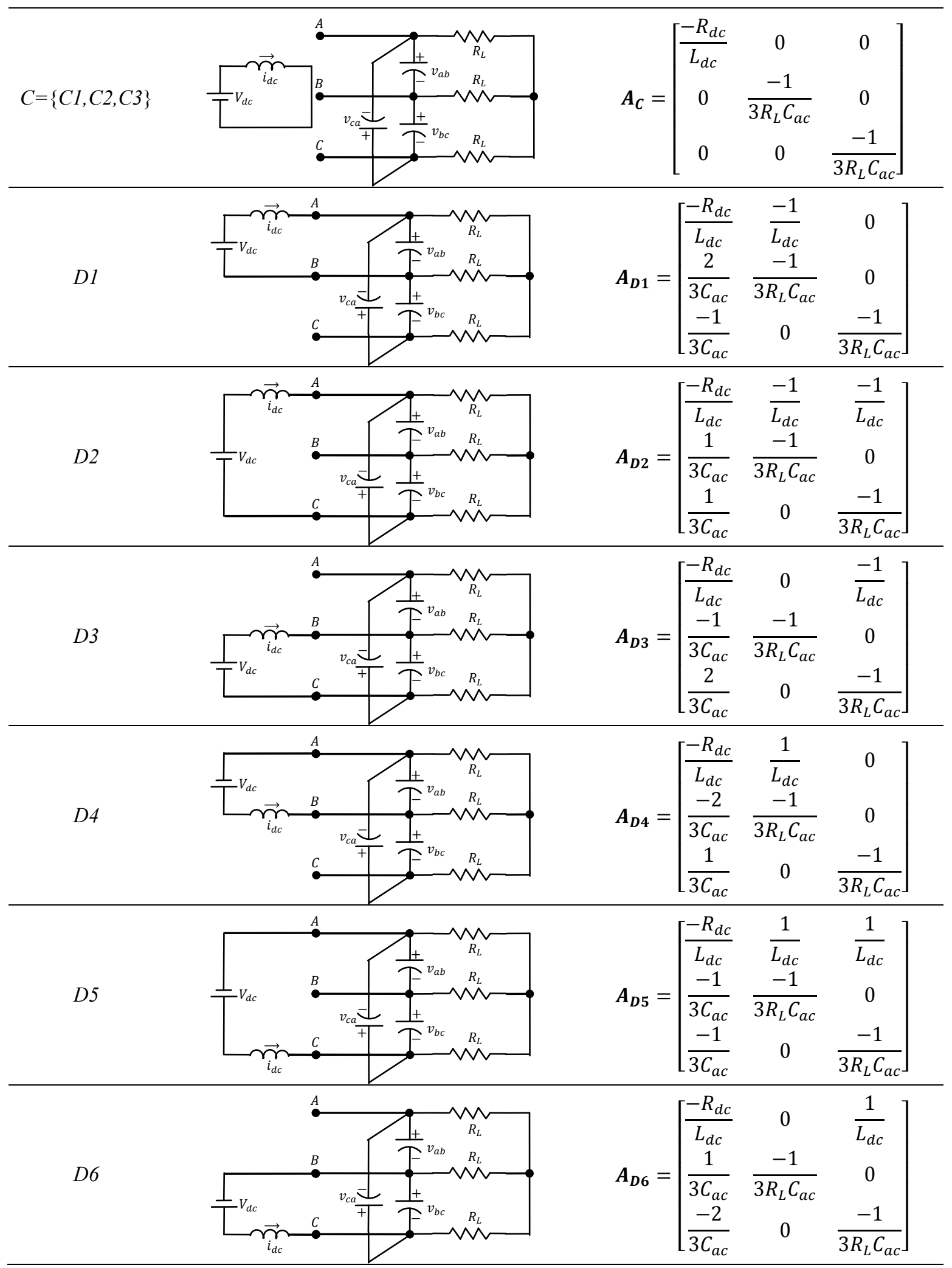




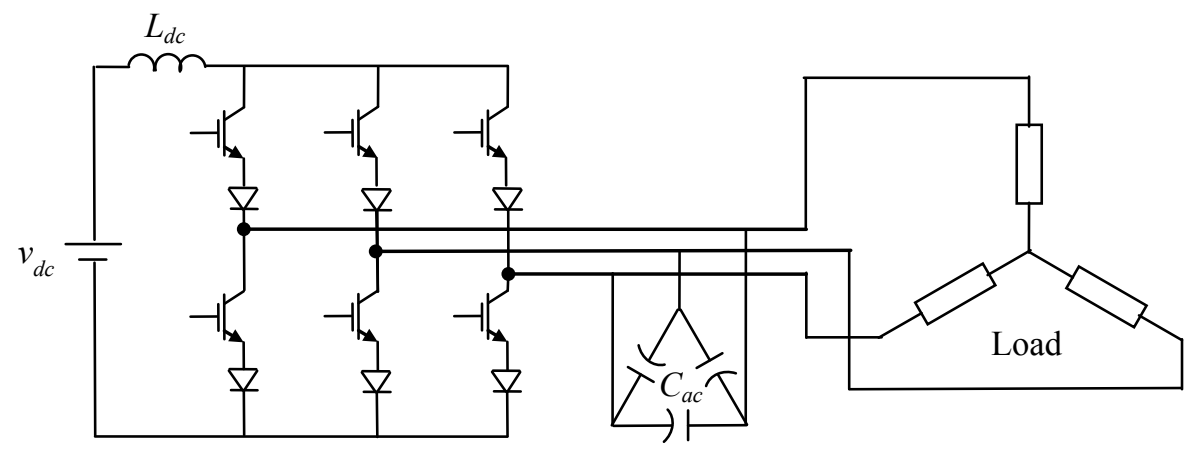

Figure 5.1: Stand-alone three-phase single-stage boost inverter system

In these state-space representations, the state vector $\boldsymbol{x}$ consists of state variables of the system, which are the inductor current and independent capacitor voltages, i.e. $\boldsymbol{x}=\left[i_{d c}, v_{a b}, v_{b c}\right]^{T}$, where the superscript $T$ denotes the matrix transpose. The dc-source voltage, $v_{d c}$, is the only input to all circuits, i.e. $\boldsymbol{u}_{C}=\boldsymbol{u}_{\boldsymbol{D} \mathbf{1}}=\boldsymbol{u}_{\boldsymbol{D} \mathbf{2}}=\cdots=\boldsymbol{u}_{\boldsymbol{D} \mathbf{6}}=\boldsymbol{u}=$ $v_{d c}$, and all states have the same control matrix, $\boldsymbol{B}_{C}=\boldsymbol{B}_{D 1}=\boldsymbol{B}_{D 2}=\cdots=\boldsymbol{B}_{\boldsymbol{D} 6}=\boldsymbol{B}=$ $\left[1 / L_{d c}, 0,0\right]^{T}$.

\subsection{State-Space-Averaged Model of the SSBI}

If one can put the time-varying differential equations of the SSBI in the standard form of (3.12) and show the periodicity and continuity of the associated vector function over a switching period, i.e. $T_{s}$, then it is possible to apply the theorem of periodic averaging (see Appendix A), and approximate the system model with a set of timeinvariant differential equations. In this study, ideal switches operating at a high switching frequency are assumed for the SSBI in order to apply the aforementioned theorem [49, $57,59]$. 


\subsubsection{Large-Signal Model}

As the first step, averaging technique is applied to the state-space equations of the stand-alone SSBI system in Sector (I). As will be seen later, all sectors will eventually have the same state-space-averaged model. The circuit diagrams and the corresponding state-space equations, under continuous conduction mode (CCM) of operation and for a resistive load, $R_{L}$, are summarized in Table 5.2, where, $R_{d c}$ is the series resistance of the dc-link inductor. It should be emphasized that in many real cases it may not be a valid assumption to neglect the series resistance. The reason is that the amount of the series resistance of the dc-link inductor may not be really negligible in comparison with the other parameters of the circuit. For instance, the dc-link inductor of our prototype circuit has a series resistance of $0.1 \Omega$. Moreover, as will be seen $\mathrm{n}$ later sections, the series resistances of the circuit elements have significant impacts on the stability of the SSBI system.

As can be seen in the table, the system matrix of the system in Sector (I), $\boldsymbol{A}_{\boldsymbol{I}}(t)$, is a function of time, however, by applying the theorem of periodic averaging [29], the system can be approximated by the following state-space equation:

$\dot{\widetilde{x}}=\bar{A}_{I} \tilde{\boldsymbol{x}}+\bar{B} \boldsymbol{u}$

where, $\widetilde{\boldsymbol{x}}=\left[\tilde{\boldsymbol{i}}_{d c}, \tilde{v}_{a b}, \tilde{v}_{b c}\right]^{T}$ is the approximate state vector, and the matrix $\overline{\boldsymbol{A}}_{\boldsymbol{I}}$ is the averaged system matrix for Sector (I),

$$
\overline{\boldsymbol{A}}_{\boldsymbol{I}}=\left[d_{c} \boldsymbol{A}_{\boldsymbol{C} \mathbf{1}}+d_{1} \boldsymbol{A}_{\boldsymbol{D} \mathbf{1}}+d_{2} \boldsymbol{A}_{\boldsymbol{D} \mathbf{2}}\right]=\left[\begin{array}{ccc}
\frac{-R_{d c}}{L_{d c}} & \frac{-\left(d_{1}+d_{2}\right)}{L_{d c}} & \frac{-d_{2}}{L_{d c}} \\
\frac{2 d_{1}+d_{2}}{3 C_{a c}} & \frac{-1}{3 R_{L} C_{a c}} & 0 \\
\frac{-d_{1}+d_{2}}{3 C_{a c}} & 0 & \frac{-1}{3 R_{L} C_{a c}}
\end{array}\right]
$$


where, $d_{c}=t_{c} / T_{s}, d_{1}=t_{d 1} / T_{s}$, and $d_{2}=t_{d 2} / T_{s}$, are the corresponding duty ratios. It should be also noted that $\overline{\boldsymbol{B}}=\boldsymbol{B}$. The averaged system matrices for other sectors, $\overline{\boldsymbol{A}}_{\boldsymbol{I I}}$, $\bar{A}_{I I I}, \bar{A}_{I V}, \bar{A}_{V}, \bar{A}_{V I}$, are provided in Table 5.3. It might be interesting to know that all of the averaged system matrices are similar, because they all have the same eigenvalues [69].

Table 5.2: Circuit diagrams and state-space equations of the SSBI under CCM, for Sector (I)

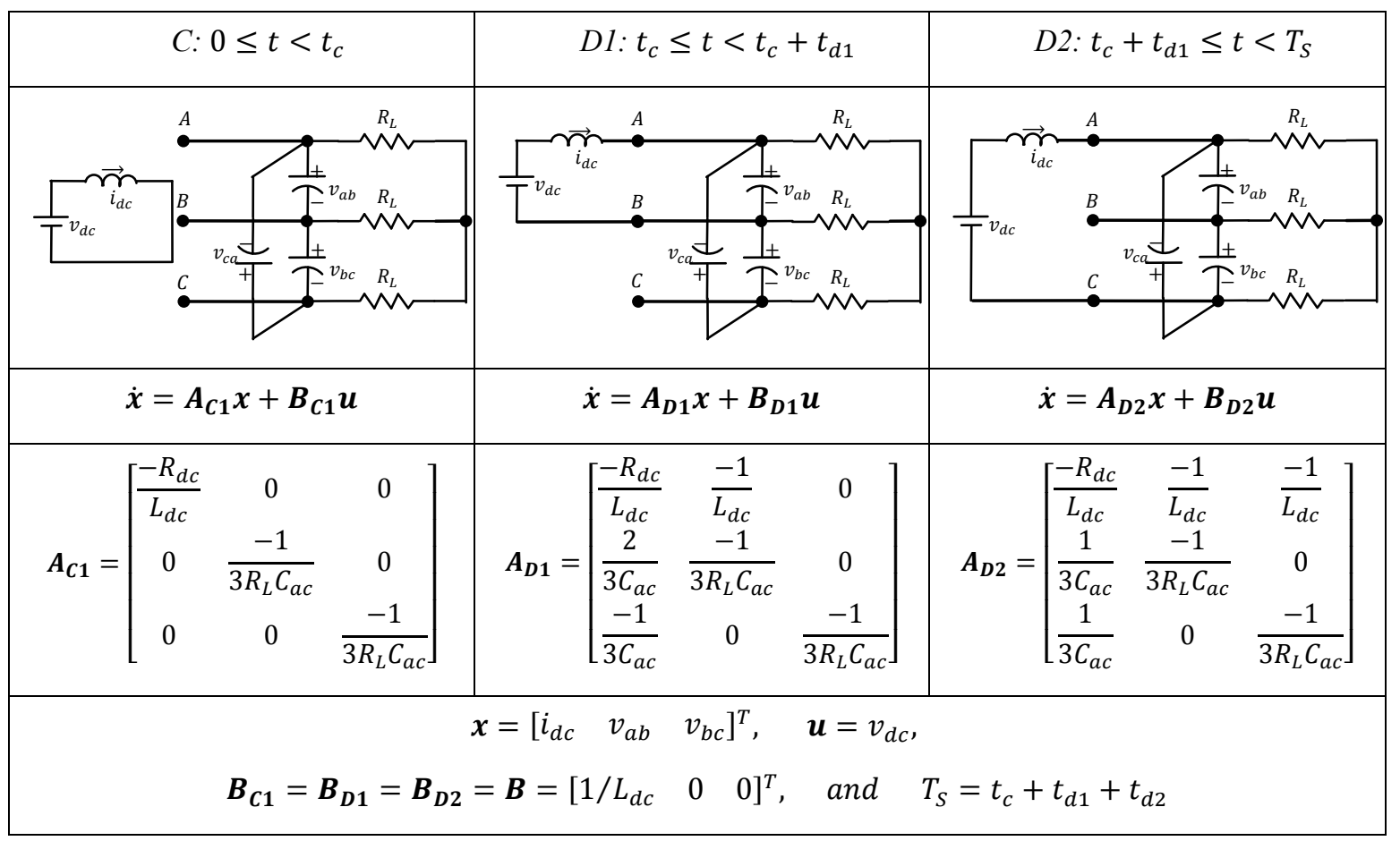

Although the obtained system matrix in (5.6) is not an explicit function of time, it is a function of discharging duty ratios, $d_{1}$ and $d_{2}$, which are varying with time. According to Chapter 4, the discharging ratios in Sector (I) are determined as:

$$
\begin{aligned}
& d_{1}=m \cos \left(\theta-\alpha_{0}\right) \\
& d_{2}=m \cos \left(\theta-\alpha_{0}-\frac{2 \pi}{3}\right)
\end{aligned}
$$


Table 5.3: Averaged system matrices of the stand-alone SSBI system for all sectors

\begin{tabular}{|c|c|c|c|c|}
\hline Sector & Average Sy & stem Matrix & & \\
\hline I & $\overline{\boldsymbol{A}}_{\boldsymbol{I}}=\left[d_{c} \boldsymbol{A}_{C}+d_{1} \boldsymbol{A}_{D 1}+d_{2} \boldsymbol{A}_{D 2}\right]=$ & {$\left[\begin{array}{c}\frac{-R_{d c}}{L_{d c}} \\
\frac{2 d_{1}+d_{2}}{3 C_{a c}} \\
\frac{-d_{1}+d_{2}}{3 C_{a c}}\end{array}\right.$} & $\begin{array}{c}-\left(d_{1}+d_{2}\right) \\
\frac{L_{d c}}{3 R_{L} C_{a c}} \\
0\end{array}$ & $\left.\begin{array}{c}\frac{-d_{2}}{L_{d c}} \\
0 \\
\frac{-1}{3 R_{L} C_{a c}}\end{array}\right]$ \\
\hline II & $\overline{\boldsymbol{A}}_{I I}=\left[d_{c} \boldsymbol{A}_{C}+d_{1} \boldsymbol{A}_{D 2}+d_{2} \boldsymbol{A}_{D 3}\right]=$ & {$\left[\begin{array}{c}\frac{-R_{d c}}{L_{d c}} \\
\frac{d_{1}-d_{2}}{3 C_{a c}} \\
\frac{d_{1}+2 d_{2}}{3 C_{a c}}\end{array}\right.$} & $\begin{array}{l}\frac{-d_{1}}{L_{d c}} \\
\frac{-1}{3 R_{L} C_{a c}} \\
0\end{array}$ & $\left.\begin{array}{c}-\left(d_{1}+d_{2}\right) \\
L_{d c} \\
0 \\
\frac{-1}{3 R_{L} C_{a c}}\end{array}\right]$ \\
\hline III & $\overline{\boldsymbol{A}}_{I I I}=\left[d_{c} \boldsymbol{A}_{C}+d_{1} \boldsymbol{A}_{D 3}+d_{2} \boldsymbol{A}_{D 4}\right]=$ & {$\left[\begin{array}{c}\frac{-R_{d c}}{L_{d c}} \\
\frac{-\left(d_{1}+2 d_{2}\right)}{3 C_{a c}} \\
\frac{2 d_{1}+d_{2}}{3 C_{a c}}\end{array}\right.$} & $\begin{array}{c}\frac{d_{2}}{L_{d c}} \\
\frac{-1}{3 R_{L} C_{a c}} \\
0\end{array}$ & $\left.\begin{array}{c}\frac{-d_{1}}{L_{d c}} \\
- \\
c \\
0 \\
\frac{-1}{3 R_{L} C_{a c}}\end{array}\right]$ \\
\hline IV & $\overline{\boldsymbol{A}}_{I V}=\left[d_{c} \boldsymbol{A}_{\boldsymbol{C}}+d_{1} A_{D 4}+d_{2} \boldsymbol{A}_{D 5}\right]=$ & {$\left[\begin{array}{c}\frac{-R_{d c}}{L_{d c}} \\
\frac{-\left(2 d_{1}+d_{2}\right)}{3 C_{a c}} \\
\frac{d_{1}-d_{2}}{3 C_{a c}}\end{array}\right.$} & $\begin{array}{c}\frac{d_{1}+d_{2}}{L_{d c}} \\
\frac{-1}{3 R_{L} C_{a c}} \\
0\end{array}$ & $\left.\begin{array}{c}\frac{d_{2}}{L_{d c}} \\
0 \\
\frac{-1}{3 R_{L} C_{a c}}\end{array}\right]$ \\
\hline V & $\overline{\boldsymbol{A}}_{\boldsymbol{V}}=\left[d_{c} \boldsymbol{A}_{C}+d_{1} \boldsymbol{A}_{D 5}+d_{2} \boldsymbol{A}_{D 6}\right]=$ & {$\left[\begin{array}{c}\frac{-R_{d c}}{L_{d c}} \\
\frac{-d_{1}+d_{2}}{3 C_{a c}} \\
\frac{-\left(d_{1}+2 d_{2}\right)}{3 C_{a c}}\end{array}\right.$} & $\begin{array}{c}\frac{d_{1}}{L_{d c}} \\
\frac{-1}{3 R_{L} C_{a c}} \\
0\end{array}$ & $\begin{array}{c}\frac{d_{1}+d_{2}}{L_{d c}} \\
0 \\
\frac{-1}{3 R_{L} C_{a c}}\end{array}$ \\
\hline VI & $\bar{A}_{V I}=\left[d_{c} \boldsymbol{A}_{C}+d_{1} \boldsymbol{A}_{D 6}+d_{2} \boldsymbol{A}_{D 1}\right]=$ & {$\left[\begin{array}{c}\frac{-R_{d c}}{L_{d c}} \\
\frac{d_{1}+2 d_{2}}{3 C_{a c}} \\
\frac{-\left(2 t_{d 1}+t_{d 2}\right)}{3 C_{a c}}\end{array}\right.$} & $\begin{array}{c}\frac{-d_{2}}{L_{d c}} \\
\frac{-1}{3 R_{L} C_{a c}} \\
0\end{array}$ & $\left.\begin{array}{c}\frac{d_{1}}{L_{d c}} \\
-\quad 0 \\
\frac{-1}{3 R_{L} C_{a c}}\end{array}\right]$ \\
\hline
\end{tabular}

where the modulation index $m>0$ is a coefficient which regulates the output voltage of the system and $\theta$ is the angular displacement of the reference line voltage, $v_{a b}^{r e f}$. Also, it is 
worth the reminder that, since $d_{1}$ and $d_{2}$ must be always positive, $\alpha_{0}$ must be equal to $-\pi / 6$

It should be mentioned that, compared with the fast variations of the system equations during a switching cycle (e.g. $T_{s}=0.1 \mathrm{msec}$ for $f_{s}=10 \mathrm{kHz}$ ), $d_{1}$ and $d_{2}$ (with a period of $1 / 60 \cong 16.7 \mathrm{msec}$ ) can be considered as relatively slow-varying quantities. In other words, in the SSBI, $v_{d c}$, and the control functions, $d_{1}$ and $d_{2}$, are dc or sinusoidal functions with slow variations in comparison with the switching cycle. Moreover, it can be concluded from the experimental results in Chapter 4 that the energy content of the switching harmonics (ripple) of the SSBI output waveforms is negligible (THD $=3.5 \%$ ). Thus, the SSBI can be viewed as a system with slow-varying inputs and slow-varying outputs [49].

As can be seen in Table 5.3, for the six sectors of the proposed phasor pulse-withmodulation (PPWM) switching pattern, there are six different sets of equations, and consequently six distinct system matrices, namely $\bar{A}_{I}, \bar{A}_{I I}, \bar{A}_{I I I}, \bar{A}_{I V}, \bar{A}_{V}, \bar{A}_{V I}$. In other words, the period of the SSBI equations is equal to the line voltage cycle, e.g. $60 \mathrm{~Hz}$. Moreover, these matrices are time-variant, as $d_{1}$ and $d_{2}$ vary by time. However, if these six state-space equations are transferred into the synchronous $d q$-frame of reference (see Chapter 3), all sectors will have the same matrix. This procedure is elaborated upon in the following.

In general, $\tilde{v}_{a b}$ and $\tilde{v}_{b c}$ can be expressed in terms of their $d q$-components, $\tilde{v}_{q}$ and $\tilde{v}_{d}$, as follows [28]:

$\left[\begin{array}{c}\tilde{v}_{a b} \\ \tilde{v}_{b c}\end{array}\right]=\left[\begin{array}{cc}\cos \left(\theta_{f}\right) & \sin \left(\theta_{f}\right) \\ \cos \left(\theta_{f}-\frac{2 \pi}{3}\right) & \sin \left(\theta_{f}-\frac{2 \pi}{3}\right)\end{array}\right]\left[\begin{array}{c}\tilde{v}_{q} \\ \tilde{v}_{d}\end{array}\right]$ 
where $\theta_{f}$ is the angular displacement of the rotating reference frame. Consequently, time derivatives of the approximate state variables $\tilde{v}_{a b}$ and $\tilde{v}_{b c}$ are derived as:

$$
\begin{aligned}
{\left[\begin{array}{c}
\dot{\tilde{v}}_{a b} \\
\dot{\tilde{v}}_{b c}
\end{array}\right] } & =\left[\begin{array}{cc}
\cos \left(\theta_{f}\right) & \sin \left(\theta_{f}\right) \\
\cos \left(\theta_{f}-\frac{2 \pi}{3}\right) & \sin \left(\theta_{f}-\frac{2 \pi}{3}\right)
\end{array}\right]\left[\begin{array}{c}
\dot{\tilde{v}}_{q} \\
\dot{\tilde{v}}_{d}
\end{array}\right] \\
+ & {\left[\begin{array}{cc}
-\dot{\theta}_{f} \sin \left(\theta_{f}\right) & \dot{\theta}_{f} \cos \left(\theta_{f}\right) \\
-\dot{\theta}_{f} \sin \left(\theta_{f}-\frac{2 \pi}{3}\right) & \dot{\theta}_{f} \cos \left(\theta_{f}-\frac{2 \pi}{3}\right)
\end{array}\right]\left[\begin{array}{c}
\tilde{v}_{q} \\
\tilde{v}_{d}
\end{array}\right] }
\end{aligned}
$$

Substituting (5.9) and (5.10) in (5.6) and some mathematical manipulations yields:

$$
\overline{\boldsymbol{A}}_{\boldsymbol{d q I}}=\left[\begin{array}{ccc}
\frac{-R_{d c}}{L_{d c}} & \frac{-\left[d_{1} \cos \left(\theta_{f}\right)+d_{2} \cos \left(\theta_{f}-\frac{\pi}{3}\right)\right]}{L_{d c}} & \frac{-\left[d_{1} \sin \left(\theta_{f}\right)+d_{2} \sin \left(\theta_{f}-\frac{\pi}{3}\right)\right]}{L_{d c}} \\
\frac{2\left[d_{1} \cos \left(\theta_{f}\right)+d_{2} \cos \left(\theta_{f}-\frac{\pi}{3}\right)\right]}{3 C_{a c}} & \frac{-1}{3 R_{L} C_{a c}} & -\dot{\theta}_{f} \\
\frac{2\left[d_{1} \sin \left(\theta_{f}\right)+d_{2} \sin \left(\theta_{f}-\frac{\pi}{3}\right)\right]}{3 C_{a c}} & \dot{\theta}_{f} & \frac{-1}{3 R_{L} C_{a c}}
\end{array}\right]
$$

which is the average system matrix of Sector (I) in the rotating reference frame.

The averaged system matrices for the other sectors, $\overline{\boldsymbol{A}}_{\boldsymbol{d q I I}}, \overline{\boldsymbol{A}}_{\boldsymbol{d q I I I}}, \ldots, \overline{\boldsymbol{A}}_{\boldsymbol{d q V I}}$, can be obtain in the same way. These matrices are presented in Table 5.4. As an alternative approach, one could apply (5.9) and (5.10) to the state-space equations of Table 5.1, see Table 5.5, and then apply the averaging technique. Equation (5.11) is still a function of $d_{1}$ and $d_{2}$. However, substituting (5.7) and (5.8) in (5.11), yields the following matrix in the rotating reference frame, where $\theta_{f}=\theta$ :

$$
\overline{\boldsymbol{A}}_{\boldsymbol{d} \boldsymbol{q} \boldsymbol{I}}=\left[\begin{array}{ccc}
\frac{-R_{d c}}{L_{d c}} & \frac{-\sqrt{3} m}{2 L_{d c}} & 0 \\
\frac{\sqrt{3} m}{3 C_{a c}} & \frac{-1}{3 R_{L} C_{a c}} & -\dot{\theta} \\
0 & \dot{\theta} & \frac{-1}{3 R_{L} C_{a c}}
\end{array}\right]
$$


Table 5.4: Averaged system matrices of the stand-alone SSBI system in the $d q$-frame of reference

\begin{tabular}{|c|c|c|c|}
\hline \multirow{6}{*}{$\bar{A}_{d q I}=$} & $\underline{-R_{d c}}$ & $-\left[d_{1} \cos \left(\theta_{f}\right)+d_{2} \cos \left(\theta_{f}-\frac{\pi}{3}\right)\right]$ & $\left.-\left[d_{1} \sin \left(\theta_{f}\right)+d_{2} \sin \left(\theta_{f}-\frac{\pi}{3}\right)\right]\right]$ \\
\hline & $\overline{L_{d c}}$ & $L_{d c}$ & $L_{d c}$ \\
\hline & $2\left[d_{1} \cos \left(\theta_{f}\right)+d_{2} \cos \left(\theta_{f}-\frac{\pi}{3}\right)\right]$ & -1 & $\dot{\theta}$ \\
\hline & $3 C_{a c}$ & $\overline{3 R_{L} C_{a c}}$ & $-\theta_{f}$ \\
\hline & $2\left[d_{1} \sin \left(\theta_{f}\right)+d_{2} \sin \left(\theta_{f}-\frac{\pi}{3}\right)\right]$ & & -1 \\
\hline & $3 C_{a c}$ & $\theta_{f}$ & $\overline{3 R_{L} C_{a c}}$ \\
\hline \multirow{6}{*}{$\bar{A}_{d q I I}=$} & $\underline{-R_{d c}}$ & $-d_{1} \cos \left(\theta_{f}-\frac{\pi}{3}\right)+d_{2} \cos \left(\theta_{f}+\frac{\pi}{3}\right)$ & $-d_{1} \sin \left(\theta_{f}-\frac{\pi}{3}\right)+d_{2} \sin \left(\theta_{f}+\frac{\pi}{3}\right)$ \\
\hline & $\overline{L_{d c}}$ & $\overline{L_{d c}}$ & $L_{d c}$ \\
\hline & $2\left[d_{1} \cos \left(\theta_{f}-\frac{\pi}{3}\right)-d_{2} \cos \left(\theta_{f}+\frac{\pi}{3}\right)\right]$ & -1 & $-\dot{\theta}_{c}$ \\
\hline & $3 C_{a c}$ & $\overline{3 R_{L} C_{a c}}$ & $-\sigma_{f}$ \\
\hline & $2\left[d_{1} \sin \left(\theta_{f}-\frac{\pi}{3}\right)-d_{2} \sin \left(\theta_{f}+\frac{\pi}{3}\right)\right]$ & $\dot{\theta}_{c}$ & -1 \\
\hline & $3 C_{a c}$ & $\boldsymbol{\sigma}_{f}$ & $3 R_{L} C_{a c}$ \\
\hline \multirow{6}{*}{$\bar{A}_{d q I I I}=$} & $\underline{-R} d c$ & $d_{1} \cos \left(\theta_{f}+\frac{\pi}{3}\right)+d_{2} \cos \left(\theta_{f}\right)$ & $d_{1} \sin \left(\theta_{f}+\frac{\pi}{3}\right)+d_{2} \sin \left(\theta_{f}\right)$ \\
\hline & $\overline{L_{d c}}$ & $\overline{L_{d c}}$ & $L_{d c}$ \\
\hline & $=-2\left[d_{1} \cos \left(\theta_{f}+\frac{\pi}{3}\right)+d_{2} \cos \left(\theta_{f}\right)\right.$ & -1 & $-\dot{\theta}_{c}$ \\
\hline & $3 C_{a c}$ & $\overline{3 R_{L} C_{a c}}$ & $-\sigma_{f}$ \\
\hline & $-2\left[d_{1} \sin \left(\theta_{f}+\frac{\pi}{3}\right)+d_{2} \sin \left(\theta_{f}\right)\right.$ & $f)]$ & -1 \\
\hline & $3 C_{a c}$ & $\boldsymbol{\sigma}_{f}$ & $\overline{3 R_{L} C_{a c}}$ \\
\hline \multirow{6}{*}{$\bar{A}_{d q I V}$} & $\underline{-R_{d c}}$ & $\underline{d_{1} \cos \left(\theta_{f}\right)+d_{2} \cos \left(\theta_{f}-\frac{\pi}{3}\right)}$ & $\left.d_{1} \sin \left(\theta_{f}\right)+d_{2} \sin \left(\theta_{f}-\frac{\pi}{3}\right)\right]$ \\
\hline & $\overline{L_{d c}}$ & $\overline{L_{d c}}$ & $L_{d c}$ \\
\hline & $-2\left[d_{1} \cos \left(\theta_{f}\right)+d_{2} \cos \left(\theta_{f}-\frac{\pi}{3}\right)\right.$ & -1 & $-\dot{\theta}_{c}$ \\
\hline & $3 C_{a c}$ & $\overline{3 R_{L} C_{a c}}$ & \\
\hline & $-2\left[d_{1} \sin \left(\theta_{f}\right)+d_{2} \sin \left(\theta_{f}-\frac{\pi}{3}\right)\right.$ & $\dot{\theta}_{c}$ & -1 \\
\hline & $3 C_{a c}$ & $\sigma_{f}$ & $\overline{3 R_{L} C_{a c}}$ \\
\hline \multirow{6}{*}{$\bar{A}_{d q V}=$} & $\underline{-R_{d c}}$ & $\underline{d_{1} \cos \left(\theta_{f}-\frac{\pi}{3}\right)-d_{2} \cos \left(\theta_{f}+\frac{\pi}{3}\right)}$ & $\underline{d_{1} \sin \left(\theta_{f}-\frac{\pi}{3}\right)-d_{2} \sin \left(\theta_{f}+\frac{\pi}{3}\right)}$ \\
\hline & $L_{d c}$ & $L_{d c}$ & $L_{d c}$ \\
\hline & $-2\left[d_{1} \cos \left(\theta_{f}-\frac{\pi}{3}\right)-d_{2} \cos \left(\theta_{f}+\frac{\pi}{3}\right)\right]$ & $\begin{array}{l}-1 \\
\end{array}$ & $-\dot{\theta}_{f}$ \\
\hline & $3 C_{a c}$ & $3 R_{L} C_{a c}$ & $\sigma_{f}$ \\
\hline & $-2\left[d_{1} \sin \left(\theta_{f}-\frac{\pi}{3}\right)-d_{2} \sin \left(\theta_{f}+\frac{\pi}{3}\right)\right]$ & & -1 \\
\hline & $3 C_{a c}$ & & $\overline{3 R_{L} C_{a c}}$ \\
\hline \multirow{6}{*}{$\overline{\boldsymbol{A}}_{d q V I}=$} & $\underline{-R_{d c}}$ & $-\left[d_{1} \cos \left(\theta_{f}+\frac{\pi}{3}\right)+d_{2} \cos (\theta)\right]$ & $\left.-\left[d_{1} \sin \left(\theta_{f}+\frac{\pi}{3}\right)+d_{2} \sin \left(\theta_{f}\right)\right]\right]$ \\
\hline & $L_{d c}$ & $L_{d c}$ & $L_{d c}$ \\
\hline & $2\left[d_{1} \cos \left(\theta_{f}+\frac{\pi}{3}\right)+d_{2} \cos \left(\theta_{f}\right)\right]$ & -1 & $-\dot{\theta}_{f}$ \\
\hline & $3 C_{a c}$ & $\overline{3 R_{L} C_{a c}}$ & $-\sigma_{f}$ \\
\hline & $2\left[d_{1} \sin \left(\theta_{f}+\frac{\pi}{3}\right)+d_{2} \sin \left(\theta_{f}\right)\right]$ & $\dot{\theta}_{f}$ & -1 \\
\hline & $3 C_{a c}$ & & $3 R_{L} C_{a c}$ \\
\hline
\end{tabular}


Table 5.5: System matrices for the switching states of the stand-alone SSBI in the $d q$-frame of reference

\begin{tabular}{|c|c|c|c|c|c|}
\hline$C=\{C 1, C 2, C 3\}$ & \multicolumn{2}{|c|}{$A_{d q C}=$} & $=\left[\begin{array}{c}\frac{-R_{d c}}{L_{d c}} \\
0 \\
0\end{array}\right.$. & $\begin{array}{cc}0 & 0 \\
\frac{-1}{3 R_{L} C_{a c}} & -\dot{\theta}_{f} \\
\dot{\theta}_{f} & \frac{-1}{3 R_{L} C_{a}}\end{array}$ & \\
\hline$D 1$ & & $A_{d q D 1}=\left[\begin{array}{l}2 \\
2\end{array}\right.$ & $\begin{array}{c}\frac{-R_{d c}}{L_{d c}} \\
\frac{2 \cos \left(\theta_{f}\right)}{3 C_{a c}} \\
\frac{2 \sin \left(\theta_{f}\right)}{3 C_{a c}}\end{array}$ & $\begin{array}{cc}\frac{-\cos \left(\theta_{f}\right)}{L_{d c}} & -s i \\
\frac{-1}{3 R_{L} C_{a c}} & - \\
\dot{\theta}_{f} & -\end{array}$ & $\left.\begin{array}{l}\frac{i n\left(\theta_{f}\right)}{L_{d c}} \\
-\dot{\theta}_{f} \\
-1 \\
R_{L} C_{a c}\end{array}\right]$ \\
\hline D2 & $A_{d q D 2}=$ & $\begin{array}{r}\frac{-R_{d c}}{L_{d c}} \\
\frac{2 \cos \left(\theta_{f}-\tau\right.}{3 C_{a c}} \\
\frac{2 \sin \left(\theta_{f}-\pi\right.}{3 C_{a c}}\end{array}$ & $\begin{aligned} \frac{-c 0}{\pi / 3)} \\
\frac{\pi / 3)}{2}\end{aligned}$ & $\begin{array}{c}\frac{\cos \left(\theta_{f}-\pi / 3\right)}{L_{d c}} \\
\frac{-1}{3 R_{L} C_{a c}} \\
\dot{\theta}_{f}\end{array}$ & $\left.\begin{array}{c}-\sin \left(\theta_{f}-\pi / 3\right) \\
L_{d c} \\
-\dot{\theta}_{f} \\
\frac{-1}{3 R_{L} C_{a c}}\end{array}\right]$ \\
\hline D3 & $A_{d q D 3}=$ & {$\left[\begin{array}{r}\frac{-R_{d}}{L_{d c}} \\
-2 \cos \left(\theta_{f}\right. \\
3 C_{a c} \\
-2 \sin \left(\theta_{f}\right. \\
3 C_{a c}\end{array}\right.$} & $\begin{array}{l}\frac{d c}{c} \\
\frac{+\pi / 3)}{a c} \\
\frac{+\pi / 3)}{a c}\end{array}$ & $\begin{array}{c}\frac{\cos \left(\theta_{f}+\pi / 3\right)}{L_{d c}} \\
\frac{-1}{3 R_{L} C_{a c}} \\
\dot{\theta}_{f}\end{array}$ & $\left.\begin{array}{c}\frac{\sin \left(\theta_{f}+\pi / 3\right)}{L_{d c}} \\
-\dot{\theta}_{f} \\
\frac{-1}{3 R_{L} C_{a c}}\end{array}\right]$ \\
\hline D4 & & $A_{d q D 4}=$ & {$\left[\begin{array}{c}\frac{-R_{d c}}{L_{d c}} \\
\frac{-2 \cos \left(\theta_{f}\right)}{3 C_{a c}} \\
\frac{-2 \sin \left(\theta_{f}\right)}{3 C_{a c}}\end{array}\right.$} & $\begin{array}{c}\frac{\cos \left(\theta_{f}\right)}{L_{d c}} \\
\frac{-1}{3 R_{L} C_{a c}} \\
\dot{\theta}_{f}\end{array}$ & $\left.\begin{array}{l}\frac{n\left(\theta_{f}\right)}{L_{d c}} \\
-\dot{\theta}_{f} \\
-1 \\
R_{L} C_{a c}\end{array}\right]$ \\
\hline D5 & $A_{d q D 5}=$ & {$\left[\begin{array}{r}\frac{-R_{d}}{L_{d c}} \\
-2 \cos \left(\theta_{f}\right. \\
3 C_{a c} \\
-2 \sin \left(\theta_{f}\right. \\
3 C_{a c}\end{array}\right.$} & $\begin{array}{l}\frac{d c}{c} \\
\frac{-\pi / 3)}{c} \\
\frac{-\pi / 3)}{c}\end{array}$ & $\begin{array}{c}\frac{\cos \left(\theta_{f}-\pi / 3\right)}{L_{d c}} \\
\frac{-1}{3 R_{L} C_{a c}} \\
\dot{\theta}_{f}\end{array}$ & $\left.\begin{array}{c}\frac{\sin \left(\theta_{f}-\pi / 3\right)}{L_{d c}} \\
-\dot{\theta}_{f} \\
\frac{-1}{3 R_{L} C_{a c}}\end{array}\right]$ \\
\hline
\end{tabular}




$$
\text { D6 } \quad \boldsymbol{A}_{\boldsymbol{d q D 6} \mathbf{6}}=\left[\begin{array}{ccc}
\frac{-R_{d c}}{L_{d c}} & \frac{-\cos \left(\theta_{f}+\pi / 3\right)}{L_{d c}} & \frac{-\sin \left(\theta_{f}+\pi / 3\right)}{L_{d c}} \\
\frac{2 \cos \left(\theta_{f}+\pi / 3\right)}{3 C_{a c}} & \frac{-1}{3 R_{L} C_{a c}} & -\dot{\theta}_{f} \\
\frac{2 \sin \left(\theta_{f}+\pi / 3\right)}{3 C_{a c}} & \dot{\theta}_{f} & \frac{-1}{3 R_{L} C_{a c}}
\end{array}\right]
$$

As was expected, the resulting state-space-averaged model of the stand-alone three-phase SSBI system is independent of the averaging period, (or the switching cycle) $T_{s}$. As can be seen in (5.12), $\dot{\theta}$ is the only time-varying term in this matrix. However, assuming a constant line voltage frequency, which is valid for stand-alone systems, makes $\dot{\theta}$ independent of time, i.e. $\dot{\theta}=\omega$. Thus, the obtained averaged system matrix is not a function of time. It can be proved that the same average matrix will be obtained for the other sectors. That is,

$$
\bar{A}_{d q I}=\bar{A}_{d q I I}=\cdots=\bar{A}_{d q V I}=\bar{A}_{d q}
$$

In the above, a resistive load was considered in the modeling of the SSBI. Similarly, the state-space-averaged model of an inductive-resistive load can also be derived. In this case, the independent phase, or inductor, currents must be also considered as state variables. Therefore, the system will be of the order of five. The state-spaceaveraged representation of this system, in which $L_{L}$ and $R_{L}$ are the load inductance and resistance, respectively, can be represented in the rotating $d q$-frame of reference as follows: 


$$
\frac{d}{d t}\left[\begin{array}{c}
\tilde{i}_{d c} \\
\tilde{v}_{q} \\
\tilde{v}_{d} \\
\tilde{i}_{q} \\
\tilde{i}_{d}
\end{array}\right]=\left[\begin{array}{ccccc}
\frac{-R_{d c}}{L_{d c}} & \frac{-\sqrt{3} m}{2 L_{d c}} & 0 & 0 & 0 \\
\frac{\sqrt{3} m}{3 C_{a c}} & 0 & -\omega & \frac{-1}{2 C_{a c}} & \frac{-\sqrt{3}}{6 C_{a c}} \\
0 & \omega & 0 & \frac{\sqrt{3}}{6 C_{a c}} & \frac{-1}{2 C_{a c}} \\
0 & \frac{1}{2 L_{L}} & \frac{-\sqrt{3}}{6 L_{L}} & \frac{-R_{L}}{L_{L}} & -\omega \\
0 & \frac{\sqrt{3}}{6 L_{L}} & \frac{1}{2 L_{L}} & \omega & \frac{-R_{L}}{L_{L}}
\end{array}\right]\left[\begin{array}{c}
\tilde{i}_{d c} \\
\tilde{v}_{q} \\
\tilde{v}_{d} \\
\tilde{i}_{q} \\
\tilde{i}_{d}
\end{array}\right]+\left[\begin{array}{c}
\frac{1}{L_{d c}} \\
0 \\
0 \\
0 \\
0
\end{array}\right] v_{d c}
$$

where $\omega$ is the angular frequency of the reference line voltage, and $\tilde{i}_{q}$ and $\tilde{i}_{d}$, are the approximate $d q$-components of phase currents. It should be noted that (5.14) represents a linear time-invariant system assuming that the modulation index, $m$ is constant.

\subsubsection{Small-Signal Model}

As one can see in (5.14), $v_{d c}$ represents the system input, and the modulation index, $m$, is assumed constant. In practice, $m$ is used to regulate the output voltage in stand-alone conditions. In this case, (5.14) will not be any longer a linear equation, but it can be linearized around any steady state operating point (see Appendix B). Let us define:

$\widetilde{\boldsymbol{x}}_{\boldsymbol{d q}}=\widetilde{\boldsymbol{X}}_{\boldsymbol{d q}}+\boldsymbol{\delta} \widetilde{\boldsymbol{x}}_{\boldsymbol{d q}}, \quad v_{d c}=V_{d c}+\delta v_{d c}, \quad m=M+\delta m$

where, $\widetilde{\boldsymbol{x}}_{\boldsymbol{d q}}=\left[\tilde{i}_{d c}, \tilde{v}_{q}, \tilde{v}_{d}, \tilde{i}_{q}, \tilde{i}_{d}\right]^{T}$ is the approximate state vector in the rotating $d q$-frame of reference, the $\delta$ denotes small-signal perturbation, and capital letters indicate the steady-state (or dc) components. Thus, the linearized (small-signal) model can be represented as follows: 
$\frac{d}{d t}\left[\begin{array}{c}\delta \tilde{i}_{d c} \\ \delta \tilde{v}_{q} \\ \delta \tilde{v}_{d} \\ \delta \tilde{i}_{q} \\ \delta \tilde{i}_{d}\end{array}\right]=\left[\begin{array}{ccccc}\frac{-R_{d c}}{L_{d c}} & \frac{-\sqrt{3} M}{2 L_{d c}} & 0 & 0 & 0 \\ \frac{\sqrt{3} M}{3 C_{a c}} & 0 & -\omega & \frac{-1}{2 C_{a c}} & \frac{-\sqrt{3}}{6 C_{a c}} \\ 0 & \omega & 0 & \frac{\sqrt{3}}{6 C_{a c}} & \frac{-1}{2 C_{a c}} \\ 0 & \frac{1}{2 L_{L}} & \frac{-\sqrt{3}}{6 L_{L}} & \frac{-R_{L}}{L_{L}} & -\omega \\ 0 & \frac{\sqrt{3}}{6 L_{L}} & \frac{1}{2 L_{L}} & \omega & \frac{-R_{L}}{L_{L}}\end{array}\right]\left[\begin{array}{c}\delta \tilde{i}_{d c} \\ \delta \tilde{v}_{q} \\ \delta \tilde{v}_{d} \\ \delta \tilde{i}_{q} \\ \delta \tilde{i}_{d}\end{array}\right]+\left[\begin{array}{cc}\frac{1}{L_{d c}} & \frac{-\sqrt{3} \widetilde{v}_{q}}{2 L_{d c}} \\ 0 & \frac{\sqrt{3} \tilde{I}_{d c}}{3 C_{a c}} \\ 0 & 0 \\ 0 & 0 \\ 0 & 0\end{array}\right]\left[\begin{array}{c}\delta v_{d c} \\ \delta m\end{array}\right]$

Equation (5.16) can be used for performing different linear control designs and small-signal stability studies of the stand-alone three-phase SSBI. For instance, one can derive the transfer functions of the system and study its frequency response through Nyquist and Bode diagrams [69].

Stability is one of the most essential concerns in design and analysis of any system. There is a variety of methods for stability analysis of power electronic converters [70-78]. The Lyapunov method can be applied to any dynamic system, however, defining a proper Lyapunov function for power converters are usually complicated [70-73]. Impedance-based stability analysis is another approach that has been addressed in the literature [74-76]. Another approach is to study the eigenvalues, or poles, of the system, in order to investigate its stability [77-78]. This method has been used in this work for stability analysis of the stand-alone SSBI system.

\subsection{Eigenvalue Sensitivity Analysis}

In this section, the eigenvalue sensitivities with respect to the system parameters are studied using the developed small-signal model of the SSBI. Herein, the state-spaceaveraged equation (5.16) is used for calculating the eigenvalues of the system. The study is based on the experimental set-up that is built in the laboratory for test and verification. 
The parameters of the developed set-up are summarized in Table 5.6. Also, the line voltage frequency is assumed constant, i.e. $\omega=120 \pi \mathrm{rad} / \mathrm{sec}$. Substituting these values into (4.14) results in the equilibrium point state vector of $\widetilde{\boldsymbol{X}}_{\boldsymbol{d q}}=[4.875,83.12,281.8,-0.1340,0.5493]^{T}$, which corresponds to a line-to-line rms voltage of $208 \mathrm{~V}$ and a line rms current of $0.4 \mathrm{~A}$. This operating point corresponds to about $145 \mathrm{~W}$, which is within the operational range of a typical PV panel. The eigenvalues of the system at this operating point are obtained as $\lambda_{1}=-96.548, \lambda_{2,3}=-67.918 \pm j 990.01$, and $\lambda_{4,5}=-148.40 \times 10^{3} \pm j 376.99$. These five eigenvalues of this system are located in the left-half $s$-plane. Therefore, the system is stable for the values given in Table 5.6. However, it does not necessarily mean that the system will be stable if the system parameters change [79]. Hence, in order to investigate the stability of the system, it is required to perform the sensitivity analysis of the system's eigenvalues to the variations of the parameters. The sensitivity of an eigenvalue $\lambda_{i}$ with respect to a system parameter, $p$, is commonly defined as follows [80]:

$S_{p}^{\lambda_{i}} \triangleq \frac{\partial \lambda_{i}}{\partial p} \frac{p}{\lambda_{i}}$

However, the real and imaginary parts of $\partial \lambda_{i} / \partial p$ are used to study the rate of the $\lambda_{i}$ movement in the $s$-plane due to the variation in $p$. Thus, $S_{p}^{R e\left(\lambda_{i}\right)}$ and $S_{p}^{\operatorname{Im}\left(\lambda_{i}\right)}$ are defined herein as follows:

$\frac{\partial \lambda_{i}}{\partial p}=\frac{\partial \operatorname{Re}\left\{\lambda_{i}\right\}}{\partial p}+j \frac{\partial \operatorname{Im}\left\{\lambda_{i}\right\}}{\partial p}=S_{p}^{\operatorname{Re}\left(\lambda_{i}\right)}+j S_{p}^{\operatorname{Im}\left(\lambda_{i}\right)}$

Meanwhile, the real parts of $\lambda_{4}$ and $\lambda_{5}$ are far from the imaginary axis. Thus, they can be considered as non-dominant eigenvalues of the system. In other words, the dynamic behavior of the system is dominated by $\lambda_{1}, \lambda_{2}$, and $\lambda_{3}$. 
Table 5.6: System parameters

\begin{tabular}{cc}
\hline Parameter & Nominal values \\
\hline$v_{d c}$ & $30 \mathrm{~V}$ \\
\hline$L_{d c}$ & $10 \mathrm{mH}$ \\
\hline$R_{d c}$ & $0.1 \Omega$ \\
\hline$C_{a c}$ & $10 \mu \mathrm{F}$ \\
\hline$L_{L}$ & $2.02 \mathrm{mH}$ \\
\hline$R_{L}$ & $300 \Omega$ \\
\hline$M$ & 0.41 \\
\hline
\end{tabular}

\section{A. Sensitivity to the DC-Source, $v_{d c}$}

As can be seen in (5.16), the system matrix is independent of $V_{d c}$, therefore, the eigenvalues of the system do not move with the variations of the dc-source voltage. Thus, the dc-source voltage does not affect the stability of the stand-alone three-phase SSBI.

B. Sensitivity to the DC-Link Inductor, $R_{d c}$ and $L_{d c}$

The impacts of $R_{d c}$ and $L_{d c}$ on the eigenvalues of the system are shown in Figures 5.2 and 5.3, respectively. As there are large gaps between the real parts of the dominant and non-dominant eigenvalues, logarithmic scales are used for the real axes. The arrows point in the directions of displacements of the eigenvalues as the associated parameter increases. In these figures, the bold cross signs $(+)$ indicate the location of the eigenvalues for the nominal values of the system's parameters in Table 5.6. In addition to the locations of the eigenvalues, the sensitivities of the real and imaginary parts of the dominant eigenvalues are plotted in the lower part of each figure. It should be noted that, 


$$
\left\{\begin{array}{l}
S_{p}^{\operatorname{Re}\left(\lambda_{3}\right)}=S_{p}^{\operatorname{Re}\left(\lambda_{2}\right)} \\
S_{p}^{\operatorname{Re}\left(\lambda_{5}\right)}=S_{p}^{\operatorname{Re}\left(\lambda_{4}\right)} \\
S_{p}^{\operatorname{Im}\left(\lambda_{1}\right)}=0 \\
S_{p}^{\operatorname{Im}\left(\lambda_{3}\right)}=-S_{p}^{\operatorname{Im}\left(\lambda_{2}\right)} \\
S_{p}^{\operatorname{Im}\left(\lambda_{5}\right)}=-S_{p}^{\operatorname{Im}\left(\lambda_{4}\right)}
\end{array}\right.
$$
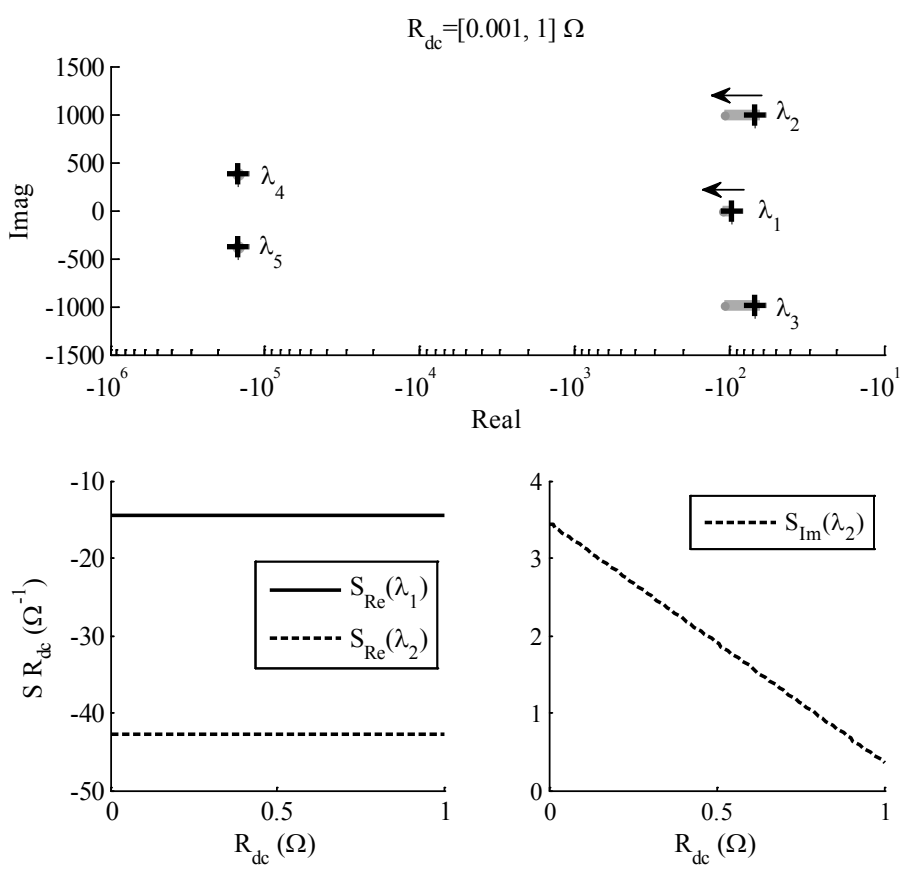

Figure 5.2: Locations of the stand-alone SSBI eigenvalues and their sensitivities versus $R_{d c}$ when it varies between 0.001 and $1 \Omega$

As can be seen in Figure 5.2, an increase in the resistance of the dc-link inductor moves the dominant eigenvalues of the system to the left, which increases the damping and speed of the system. But, it should be kept in mind that any increase in the system's resistance leads to an increase in the losses, which is not desired in power converters. 

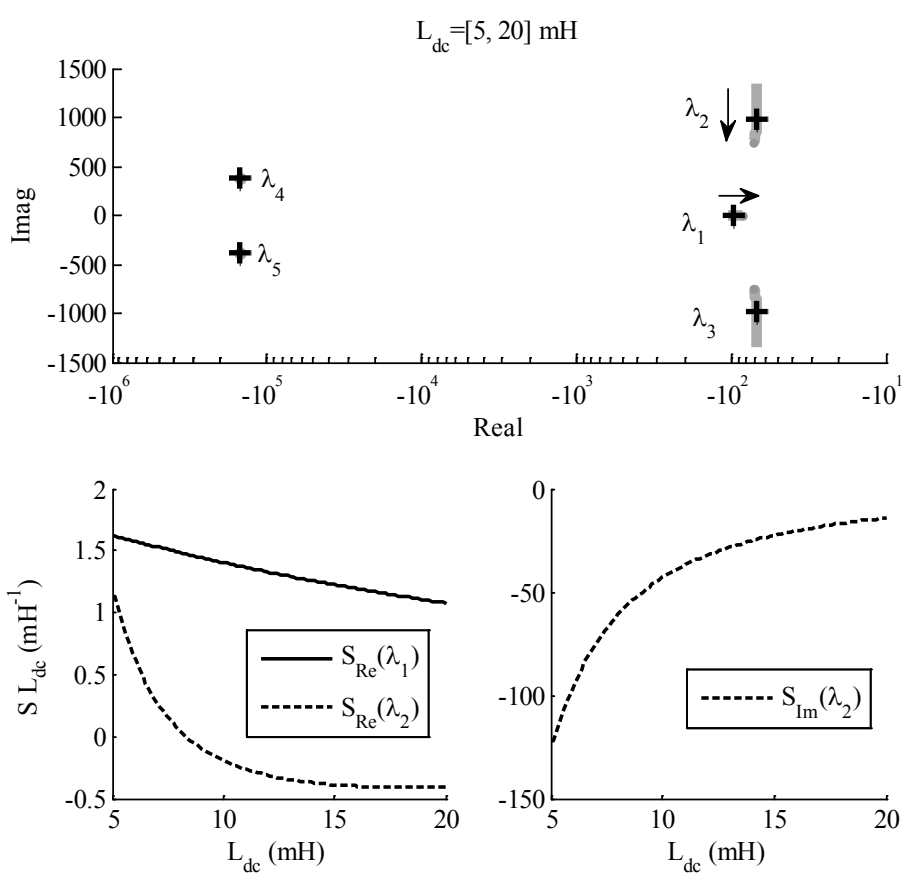

Figure 5.3: Locations of the stand-alone SSBI eigenvalues and their sensitivities versus $L_{d c}$ when it varies between 5 and $20 \mathrm{mH}$

Similarly, it can be seen in Figure 5.3 that as the inductance value of the dc-link inductor increases, $\lambda_{2}$ and $\lambda_{3}$ move toward the real axis. This results in decreases in the system natural frequencies. Consequently, the system response frequency and overshoot reduce. Additionally, it can be observed that $\lambda_{1}$ moves slightly to the right, which may slow down the system response. As one can observe from Figures 5.2 and 5.3, the impacts of the dc-link parameters, $R_{d c}$ and $L_{d c}$, on the non-dominant eigenvalues, $\lambda_{4}$ and $\lambda_{5}$, are negligible.

Notice that a negative value for $S_{p}$ means that as $p$ increases, the magnitude of the real (or the imaginary) part of the eigenvalue decreases. Also, the higher value of $\left|S_{p}\right|$ 
means the more sensitivity of the real (or the imaginary) part of the eigenvalue to the parameter variation.
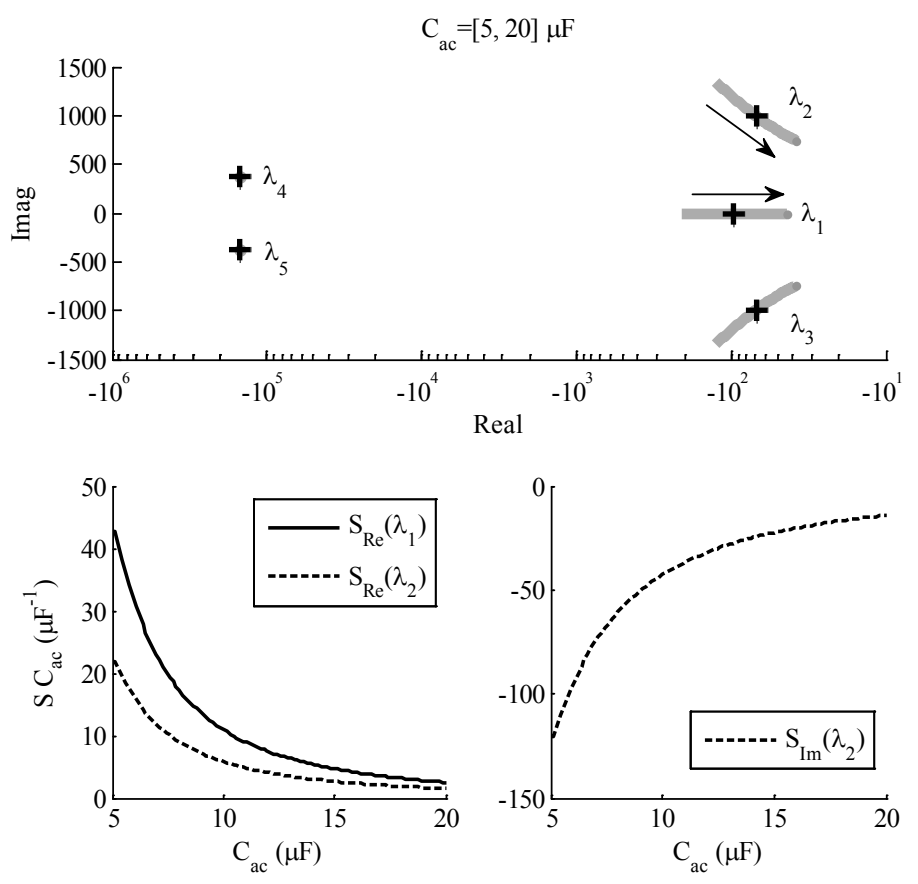

Figure 5.4: Locations of the stand-alone SSBI eigenvalues and their sensitivities versus $C_{a c}$ when it varies between 5 and $20 \mu F$

\section{Sensitivity to the AC Capacitors, $C_{a c}$}

The location of the system eigenvalues and their sensitivities to the ac-side capacitance, $C_{a c}$, are shown in Figure 5.4, where the dominant eigenvalues move toward the origin as the capacitance value increases. This reduces the system natural frequencies as well as the damping factor of the system. For instance, as $C_{a c}$ varies from 5 to $20 \mu F$, the magnitude of the real parts of $\lambda_{2}$ and $\lambda_{3}$ reduces from 100 to 40 while their imaginary part absolute values reduce from 1400 to $700 \mathrm{rad} / \mathrm{sec}$. 
D. Sensitivity to the Load Parameters, $R_{L}$ and $L_{L}$

As can be seen in Figure 5.5, increasing the load resistance moves the dominant eigenvalues of the three-phase SSBI to the right-half $s$-plane and the non-dominant eigenvalues toward the left. In general, increasing the load resistance aggravates the stability of the stand-alone SSBI.
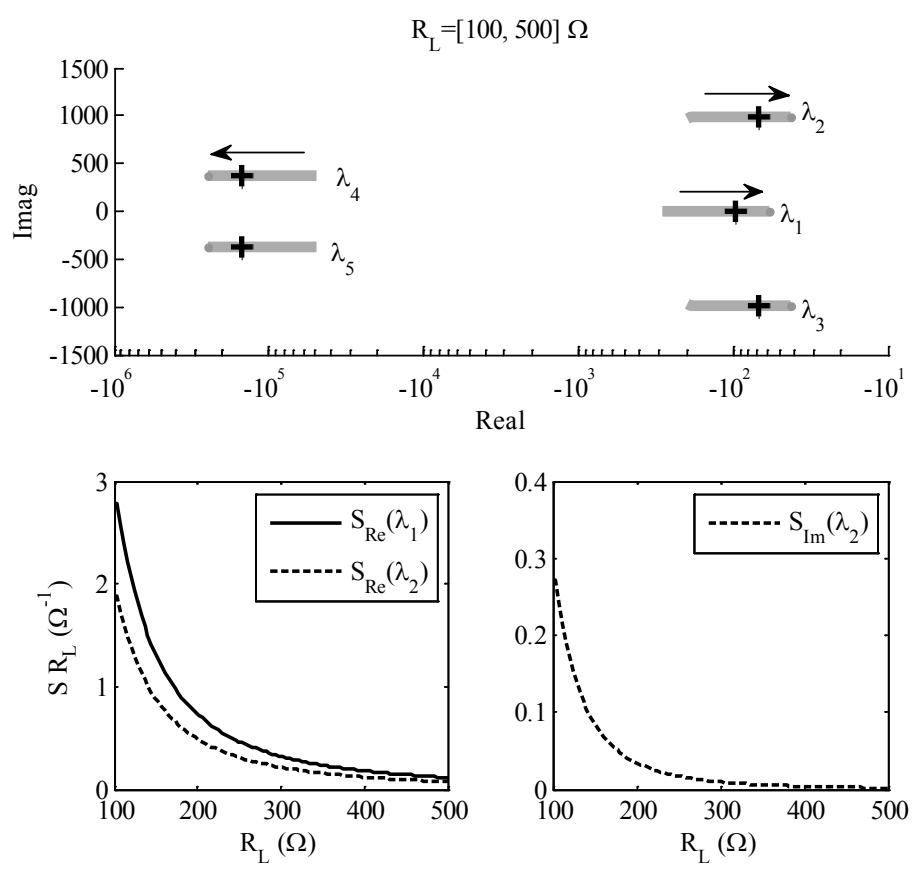

Figure 5.5: Locations of the stand-alone SSBI eigenvalues and their sensitivities versus $R_{L}$ when it varies between 100 and $500 \Omega$

The impact of the load inductance, $L_{L}$, on the location of the systems's eigenvalues is demonstrated in Figure 5.6. It is interesting that the inductive component of the load does not affect the dominant eigenvalues of the system, whereas it makes the nondominant eigenvalues to move toward the right-half s-plane. However, the magnitudes of 
their real parts are significantly larger than the dominant eigenvalues. Thus, one can conclude that the impact of the load inductance value on the three-phase SSBI dynamic is negligible.
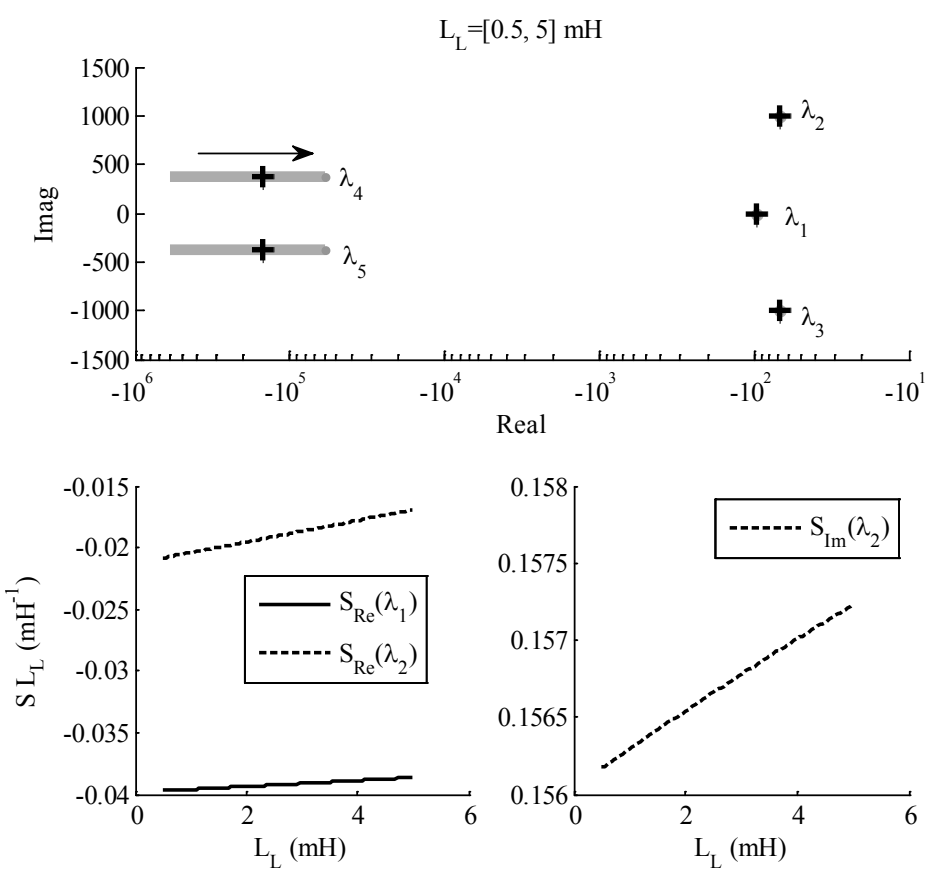

Figure 5.6: Locations of the stand-alone SSBI eigenvalues and their sensitivities versus $L_{L}$ when it varies between 0.5 and $5 \mathrm{mH}$

\section{E. Sensitivity to the Modulation Index, $M$}

The location of system's eigenvalues as the modulation index varies between 0.25 and 0.50 is shown in Figure 5.7. As can be seen, the absolute values of the imaginary parts of $\lambda_{2}$ and $\lambda_{3}$ increase whereas the real part absolute values decrease as $M$ increases. Unlike $\lambda_{2}$ and $\lambda_{3}, \lambda_{1}$ moves away from the imaginary axis as $M$ increases. However, since the pair eigenvalues, $\lambda_{2}$ and $\lambda_{3}$, have higher weights than the single one, it can be expected that the overall system response becomes more oscillatory as $M$ increases. 

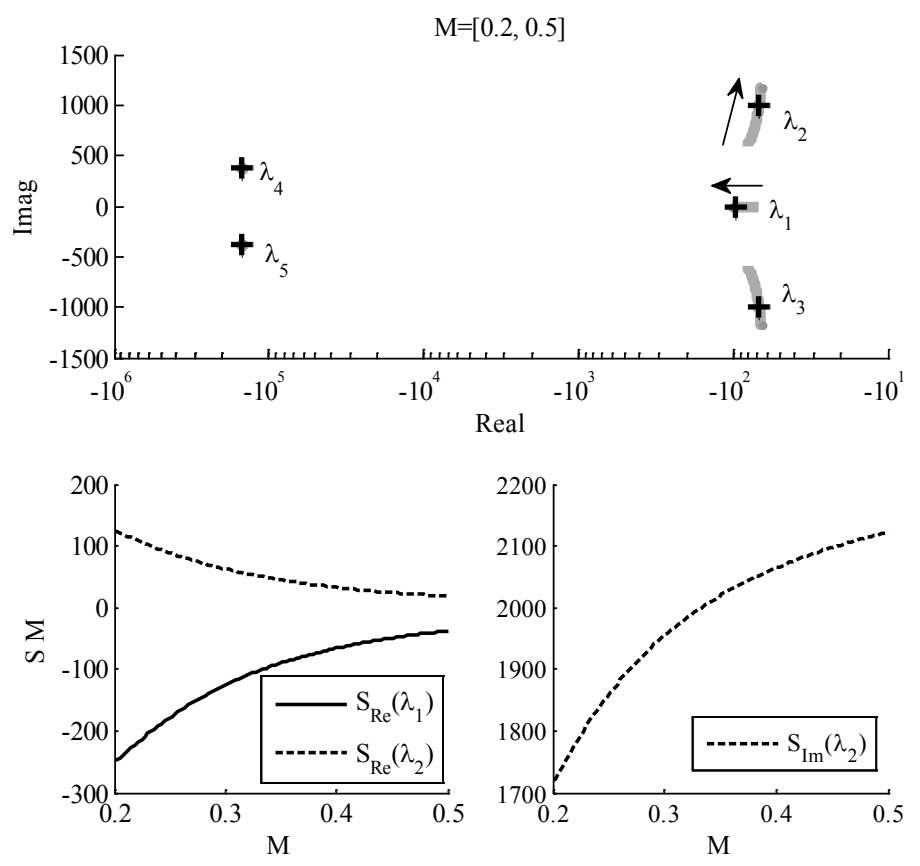

Figure 5.7: Locations of the stand-alone SSBI eigenvalues and their sensitivities versus $M$ when it varies between 0.2 and 0.5

\subsection{Simulation and Experimental Results}

In order to verify the validity of the developed state-space-averaged model, a laboratory-scaled three-phase SSBI, feeding an RL load, has been modeled and simulated in MATLAB/Simulink. The parameters of the developed set-up are summarized in Table 5.6. The inverter is switched at a frequency of $f_{s}=2880 \mathrm{~Hz}$. These switching signals are calculated and produced using a dSPACE 1104, which operates at a sampling frequency of $f_{\text {sam }}=86 \mathrm{kHz}$. It should be noted that the switching and sampling frequencies play key roles in both quality of the produced waveforms and accuracy of the developed statespace-averaged model. In other words, as the switching frequency increases the high 
frequency ripples of the input and output waveforms decrease. As a result, for the same operating point, the peak of the input dc current, $i_{d c}$, and the total harmonic distortion (THD) of the load voltage and current decreases.

Similarly, as the ratio of the sampling frequency to the switching frequency, $f_{\text {sam }} / f_{s}$, increases, the accuracy of the modulation increases. The reason is that the duration of each switching (high or low) signal must be an integer multiple of the sampling frequency. For example when this ratio is equal to $20 \%, d_{1}$ can only be modulated as multiples of $5 \%$, i.e. $5 \%$ resolution. In other words, if $d_{1}$ is desired to be $22 \%$ from (5.7), the output signal can be modulated at either $20 \%$ or $25 \%$ of duty ratio. Our studies show that the discrepancy between the desired (ideal) and the produced (actual) duty ratios causes some distortions, e.g. low order harmonics and oscillation in the inverter input/output waveforms. The effects of switching and sampling frequencies on the quality of the produced waveforms and accuracy of the averaged model are illustrated in Figures 5.8 and 5.9.

\section{A. Simulation Results}

The dynamic behavior of the three-phase SSBI during start-up and its response to step changes of the dc-source voltage, $v_{d c}$, and the modulation index, $m$, are shown in Figure 5.8. First, the system starts with the nominal parameters of Table 5.6. Second, at $t=60 \mathrm{msec}$, a $5 \mathrm{~V}$ step is applied to the dc-source voltage, i.e. $v_{d c}$ increases from 30 to $35 \mathrm{~V}$. Third, at $t=100 \mathrm{msec}$, a $3 \%$ step change is applied to the modulation index, i.e. $\mathrm{m}$ increases from 0.41 to 0.44 . 

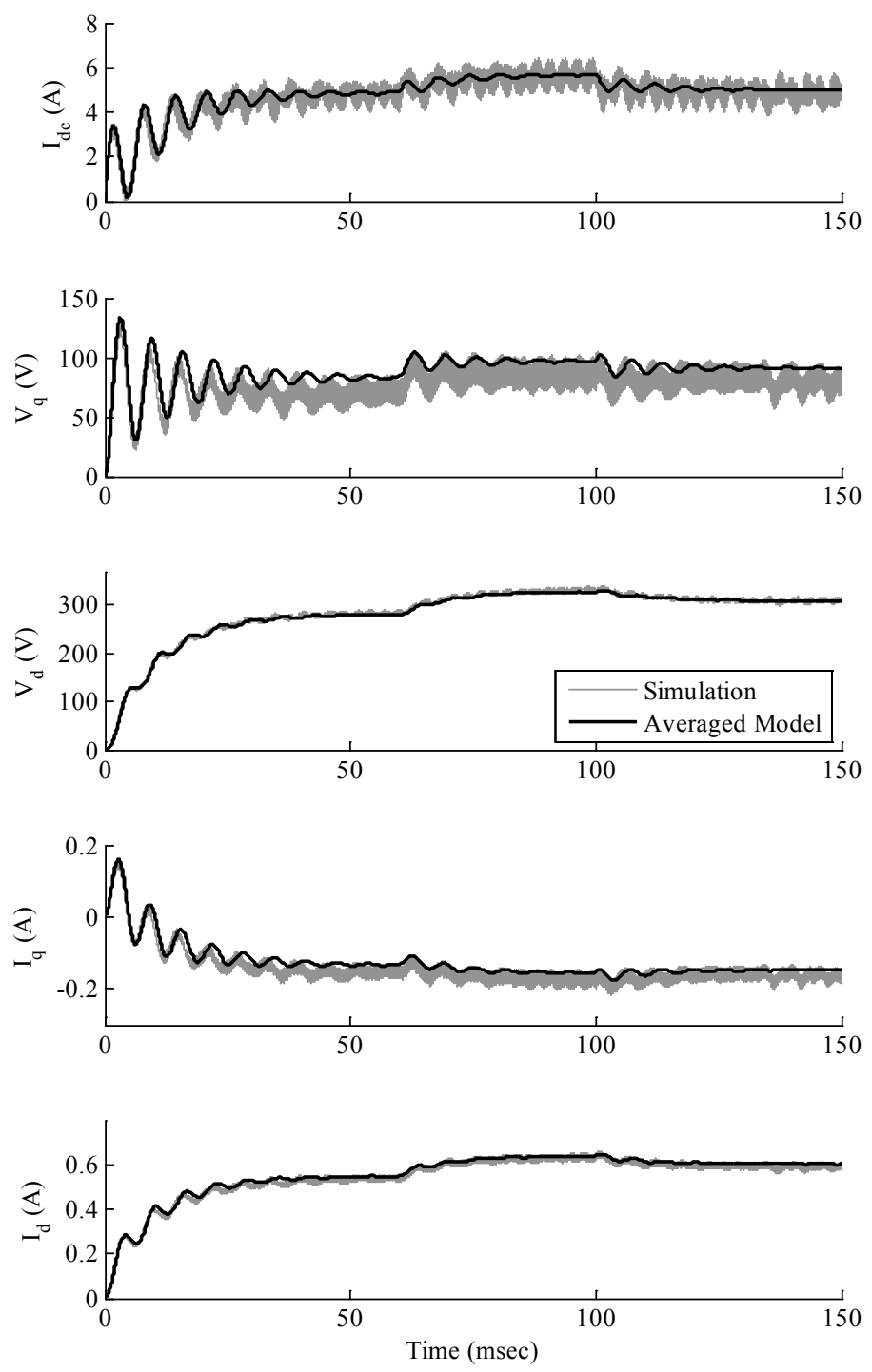

Figure 5.8: State variables of the simulation and state-space-averaged model for $f_{s}=2.88 \mathrm{kHz}$ and

$$
f_{\text {sam }}=86 \mathrm{kHz}
$$

It can be seen that the state-space-averaged model can adequately represent the dynamic and steady-state behavior of the stand-alone three-phase SSBI. It should be noted that the small-signal model can satisfactorily represent the dynamics of the system during its start-up operation, which is a large-signal disturbance in the dc-source voltage. The 
reason is that the system and input matrices of the system (5.14), are independent of $v_{d c}$. In other words, the system is linear with respect to $v_{d c}$. Therefore, as long as the other parameters of the system, including $m$, do not change, the large- and small-signal transfer functions of the system are the same. Accordingly, the small-signal model of (5.16) can also be used for dynamic analysis of the system under large-signal disturbances in the dcsource voltage.

In order to investigate the impact of the switching and sampling frequencies on the agreement between the state-space averaged model and the circuit simulation, the same simulation has been repeated for two other cases, Case $(i): f_{s}=2.88 \mathrm{kHz}$ and $f_{\text {sam }}=$ $288 \mathrm{kHz}$, and Case (ii): $f_{s}=10 \mathrm{kHz}$ and $f_{\text {sam }}=1000 \mathrm{kHz}$.

The state variables of the system for Case (ii) are shown in Figure 5.9. A comparison between Figure 5.8 and 5.9. indicates that the increase in the sampling rate significantly improves the agreement between the state-space-averaged model and simulated data. The reader should note that the outputs of the averaged model are independent of both of the switching and sampling frequencies. In fact, as has been mentioned earlier, the averaging method is valid when the switching frequency is sufficiently high, so that the small-ripple condition is satisfied and the inverter operates in a continuous conduction mode. Herein, it is observed that the sampling frequency can also affect the validity of the state-space averaging method, i.e. the higher sampling frequency, the less difference between the averaged model and real data. The mean absolute errors of the state variables of the stand-alone three-phase SSBI during the $150 \mathrm{msec}$ of the simulation are presented in Table 5.7. The error of each state variable $x$ has been defined as the difference between the simulated and approximated values: 

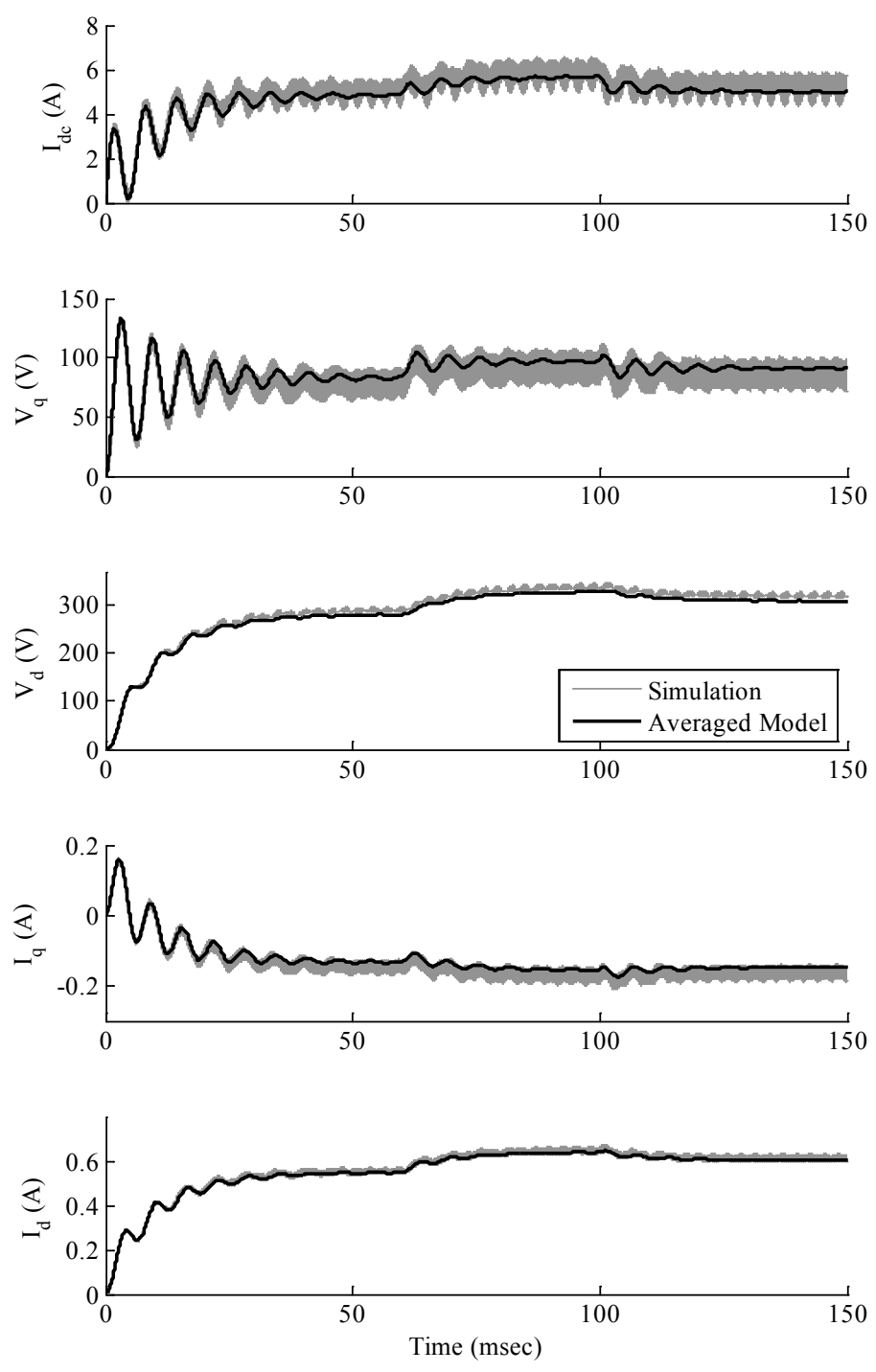

Figure 5.9: State variables of the simulation and state-space-averaged model for $f_{s}=2.8 \mathrm{kHz}$ and $f_{\text {sam }}=$ $280 \mathrm{kHz}$

$\operatorname{MAE}(x)=\frac{1}{N} \sum_{n=1}^{N}\left|x_{n}-\tilde{x}_{n}\right|$

where $N$ denotes the total number of samples over the case study period, i.e. $150 \mathrm{msec}$. It can be observed that at $f_{s}=10 \mathrm{kHz}$ and $f_{\text {sam }}=1000 \mathrm{kHz}$, the state-space-averaged model follows the simulation results with a relatively high precision. 
Table 5.7: Mean Absolute error of the state-space-averaged model for different switching and sampling frequencies

\begin{tabular}{cccc}
\hline State Variable & $\begin{array}{c}f_{s}=2.88 \mathrm{kHz} \\
f_{\text {sam }}=86 \mathrm{kHz}\end{array}$ & $\begin{array}{c}f_{s}=2.88 \mathrm{kHz} \\
f_{\text {sam }}=288 \mathrm{kHz}\end{array}$ & $\begin{array}{c}f_{s}=10 \mathrm{kHz} \\
f_{\text {sam }}=1000 \mathrm{kHz}\end{array}$ \\
\hline$M A E\left(i_{d c}\right)$ & $0.308 \mathrm{~A}$ & $0.335 \mathrm{~A}$ & $0.099 \mathrm{~A}$ \\
\hline$M A E\left(v_{q}\right)$ & $11.8 \mathrm{~V}$ & $7.35 \mathrm{~V}$ & $3.83 \mathrm{~V}$ \\
\hline$M A E\left(v_{d}\right)$ & $3.13 \mathrm{~V}$ & $7.38 \mathrm{~V}$ & $1.11 \mathrm{~V}$ \\
\hline$M A E\left(i_{q}\right)$ & $0.020 \mathrm{~A}$ & $0.0162 \mathrm{~A}$ & $0.0063 \mathrm{~A}$ \\
\hline$M A E\left(i_{d}\right)$ & $0.0121 \mathrm{~A}$ & $0.0099 \mathrm{~A}$ & $0.0042 \mathrm{~A}$ \\
\hline
\end{tabular}

\section{B. Experimental Results}

The state-space-averaged models of (5.14) and (5.16) have been derived for an ideal three-phase SSBI, in which all voltage drops and delays of the semiconductor devices (switches and diodes) have been neglected. However, in practice, these devices do not behave perfectly. For example, there are conduction losses and voltage drops across the semiconductor switches and diodes during conductions. Also, turn-on and turn-off transitions have some delays, and the diodes have reverse-recovery currents. Additionally, in order to protect the semiconductor switches and diodes against voltage spikes, they must be equipped with some turn-off snubbers (herein, RDC snubbers). Although, the snubbers suppress the voltage spikes, they may increase the losses and change the switching behavior of the semiconductor devices. All these non-idealities affect the accuracy of the derived state-space-averaged model. Hence, it would be great if the averaged model could be modified in a way that the effects of the aforementioned nonidealities could be included. Our extensive studies showed that the voltage drops and losses of the semiconductor devices could be represented by a change at the dc-source 
voltage, $v_{d c}$. Similarly, it was observed that the effects of the snubbers and imperfect switching would be incorporated into the averaged model through a slight change in the modulation index, $m$.

The dynamics of the start-up operation (large-signal disturbance in the dc-source voltage) of the laboratory-scaled three-phase SSBI, along with the response of the modified state-space-averaged model, have been depicted in Figures 5.10 through 5.12, and the hardware data is presented in Table 5.8.

Table 5.8: Hardware specifications

\begin{tabular}{cc}
\hline Component & Specifications \\
\hline dc-source & XR125-32 Magna-Power Electronics \\
\hline MOSFETS & STx42N65M5 (TO-220) \\
\hline Diodes & DSEP30-06A (TO-247 AD) \\
\hline Simulink-Hardware Interface & dSPACE 1104 \\
\hline$C_{\text {snubber }}$ & $40 \mathrm{nF}$ \\
\hline$D_{\text {snubber }}$ & $1 \mathrm{~N} 4004-\mathrm{G}$ \\
\hline$R_{\text {snubber }}$ & $240 \Omega$ \\
\hline
\end{tabular}

The aforementioned modifications can be applied as follows:

i) The dc-source voltage of the model must be $5 \mathrm{~V}$ lower than the real dcsource voltage in order to take into account the voltage drops and losses across the semiconductor devices as well as the snubber effects.

ii) The modulation index must increase by $1 \%$ in the model to include the delay and imperfect switching effects.

In other words, the experimental waveforms of the stand-alone three-phase SSBI system with a dc-source voltage of $v_{d c}=35 \mathrm{~V}$ and a modulation index of 
$m=0.40$ correspond to the waveforms of the state-space-averaged model for $v_{d c}=30 \mathrm{~V}$ and $m=0.41$.
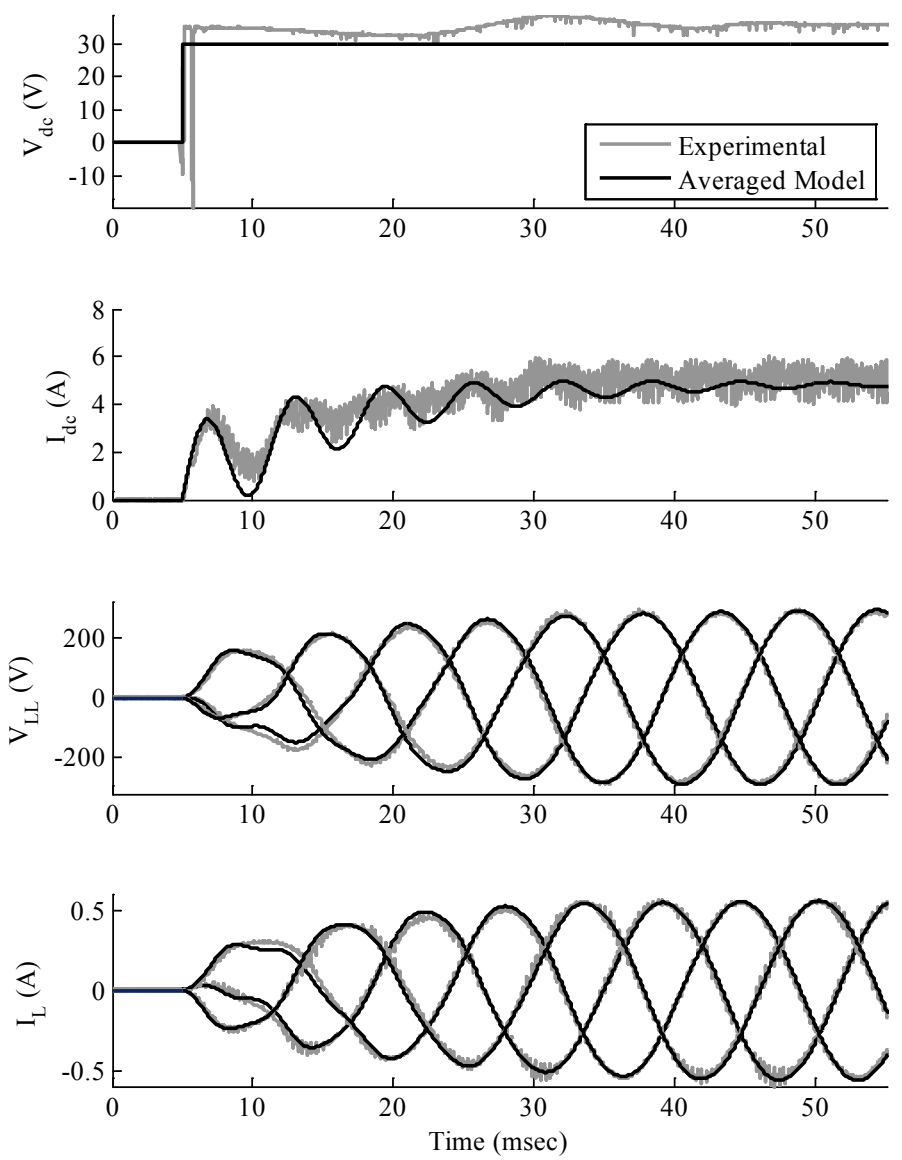

Figure 5.10: Start-up operation of the lab-scaled circuit and the response of the state-space-averaged model for $f_{s}=2.88 \mathrm{kHz}$ and $f_{\text {sam }}=86 \mathrm{kHz}$

The experimentally obtained data from the prototype circuit and the state-spaceaveraged model are plotted in Figure 5.10 and 5.11. From these figures, one can observe that the outputs of the modified state-space-averaged model are in an acceptable agreement with the experimentally obtained data. The minor differences between the 
model and real data might be due to the inherent limitations of the state-space averaging modeling, as well as unconsidered non-idealities such as the imperfect switching of the dcsource, low sampling frequency, and the parasitic and unbalanced elements of the circuit. The differences may become more noticeable in the small-signal response of the system, in which the variations of the state-variables are comparable with the ripples and distortions. This can be realized in Figure 5.12, where the response of the prototyped three-phase SSBI to a small-signal disturbance (a 3\% step change) in the modulation index, $m$, has been presented. However, the model response is still reasonably acceptable for many applications.
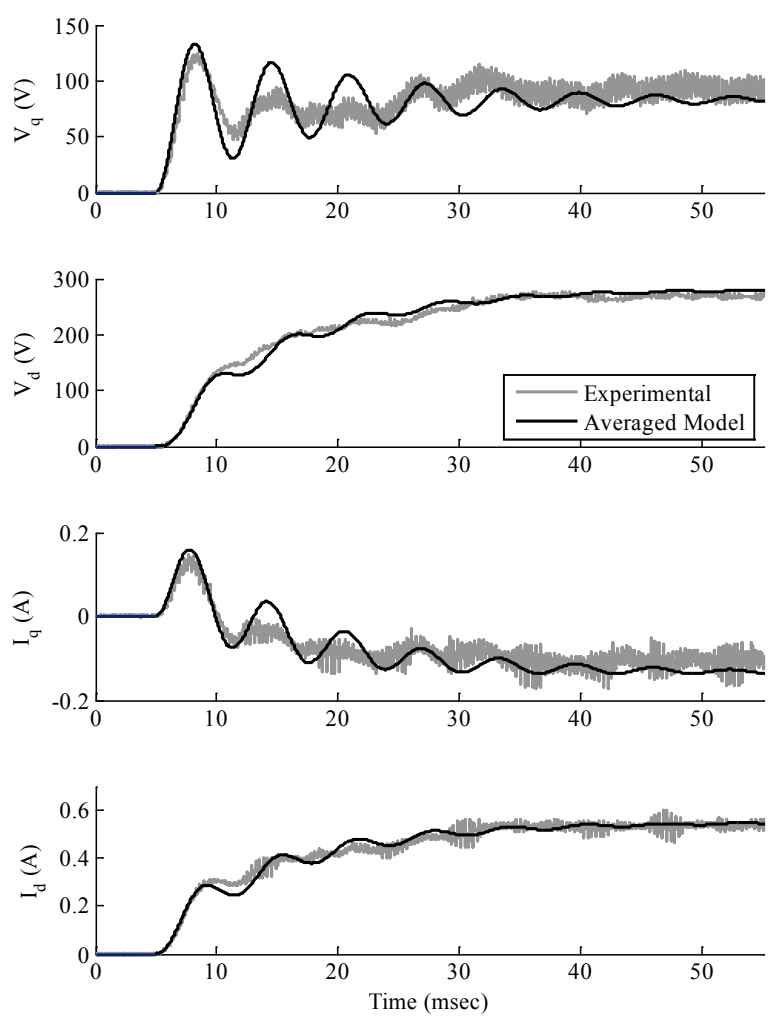

Figure 5.11: $d q$ state variables of the lab-scaled circuit and the state-space-averaged model during the startup for $f_{s}=2.88 \mathrm{kHz}$ and $f_{\text {sam }}=86 \mathrm{kHz}$ 

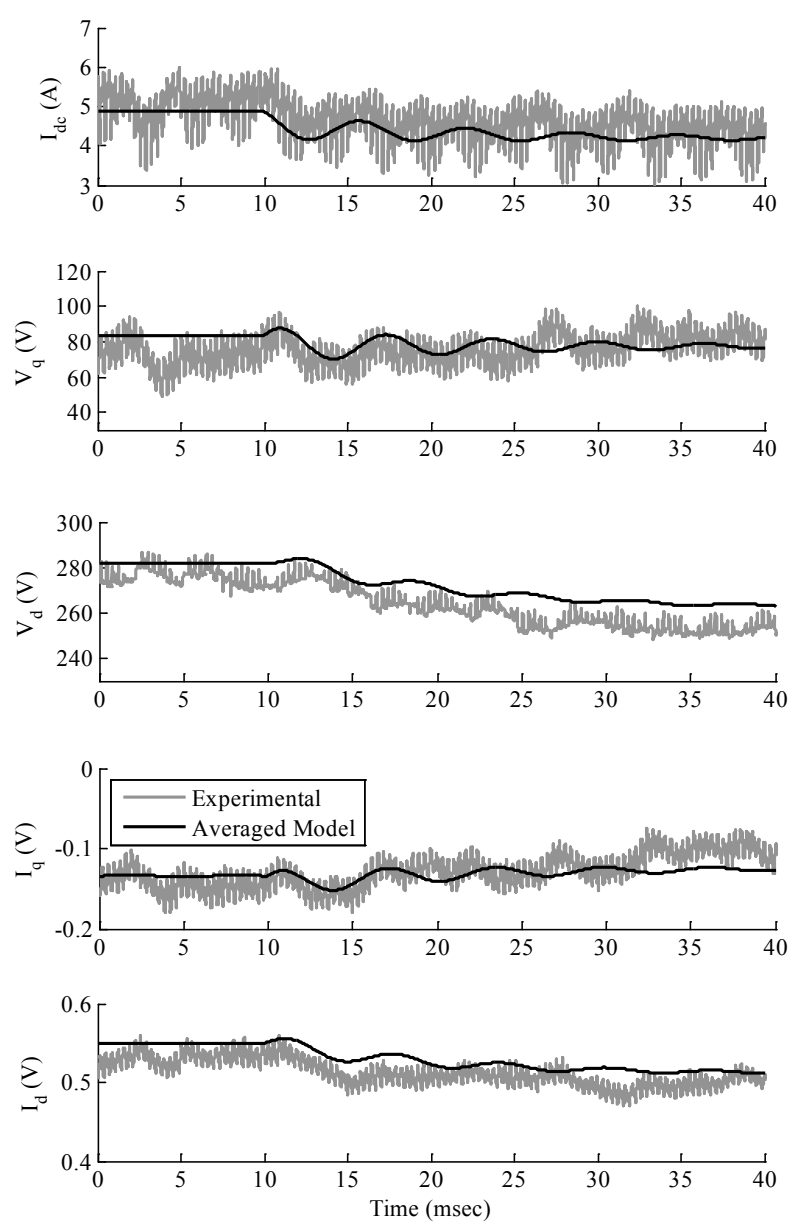

Figure 5.12: Step responses of the hardware and the state-space-averaged model, to a $3 \%$ increase in $m$, for

$$
f_{s}=2.88 \mathrm{kHz} \text { and } f_{\text {sam }}=86 \mathrm{kHz}
$$

\subsection{Summary}

The state-space averaging method has been applied in order to develop the dynamic model of the stand-alone three-phase single-stage boost inverter. The large- and small-signal models have been derived in the synchronous $d q$-frame of reference, and used for sensitivity and stability analysis of the system. It was observed that the standalone three-phase single-stage inverter system has three dominant and two non-dominant eigenvalues, in which the location of the non-dominant eigenvalues are only affected by 
the load parameters. A modification approach for taking into account the losses and nonidealities of the semiconductor devices, has been proposed and has been validated through simulated and experimentally obtained data. The large- and small-signal models of the grid-connected three-phase SSBI will be derived and analyzed in the next chapter. 


\section{CHAPTER 6}

\section{STATE-SPACE-AVERAGED MODEL OF THE GRID-CONNECTED SINGLE- STAGE BOOST INVERTER SYSTEM}

In this chapter, the state-space averaging technique is applied to derive the largeand small-signal models of the grid-connected single-stage boost inverter. These models are useful for stability analysis and control design of the system. The possible switching states of the circuit operation are enumerated and the associated state-space equations are

derived, averaged, and transformed into the synchronous $d q$-frame of reference in order to obtain a time-invariant model for the system. The system stability is investigated through eigenvalue sensitivity analysis and simulation and experimental results verify the obtained models.

This chapter contains five sections. The states of the system are enumerated in Section 1. The state-space-averaged model of the system is derived in Section 2. The eigenvalue sensitivity analysis of the system is performed in Section 3. The simulation and experimental results are presented in Section 4, and Section 5 is the summary of the chapter.

\subsection{State Enumeration of the System}

The circuit diagram of a grid-connected three-phase single-stage boost inverter (SSBI) is presented in Figure 6.1. As can be seen in this figure, the SSBI is connected to the grid through a grid interconnecting inductor, $L_{g}$, which is required for power flow control as well as filtering the injected current to the grid. Like the stand-alone SSBI, this 
system also operates in six sectors. Each sector has three switching states, one for charging and the other two for discharging. These switching states include a total of nine linear circuits, in which the three charging states are electrically the same, and the other six discharging circuits correspond to different circuitries. Therefore, seven distinct linear electric circuits are associated with the operation of the SSBI. Since every linear circuit can be represented by a set of state-space equations, the SSBI will have seven state-space equations in total, and three state-space equations over each switching cycle. Therefore, the SSBI is a linear time-varying system, which can be represented by (5.1).

The seven circuit diagrams, along with the corresponding system matrices for the grid-connected three-phase SSBI, are presented in Table 6.1, where, $R_{d c}$ and $R_{g}$ are the series resistance of the dc-link and grid interconnecting inductor, respectively. It is observed that all discharging states, i.e. $D 1-D 6$, have the same eigenvalues, which means that they are all similar matrices [69].

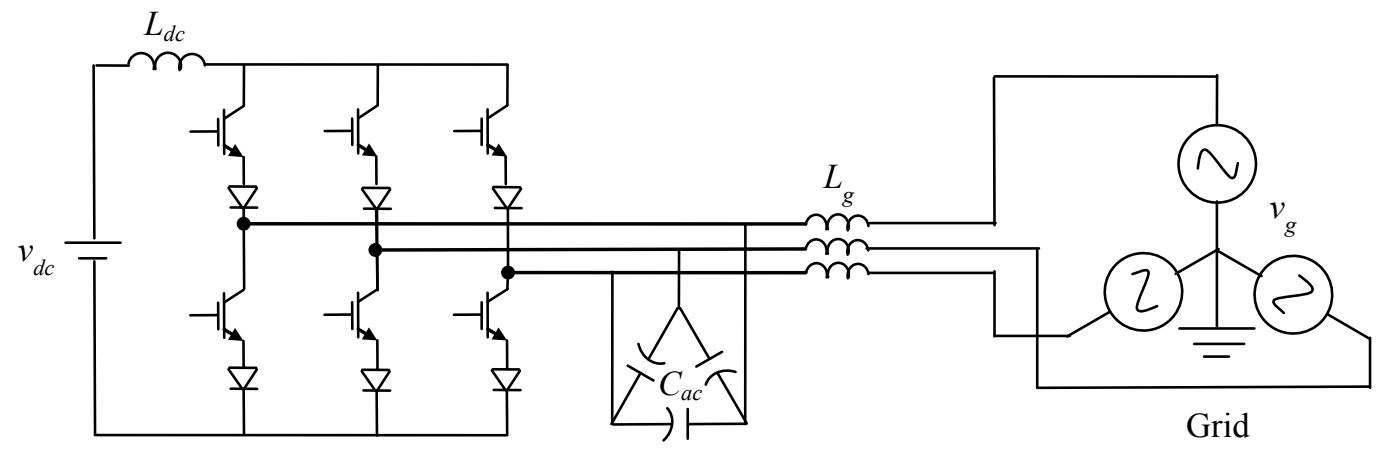

Figure 6.1: Grid-connected three-phase single-stage boost inverter system 
Table 6.1: The possible states of the grid-connected SSBI system
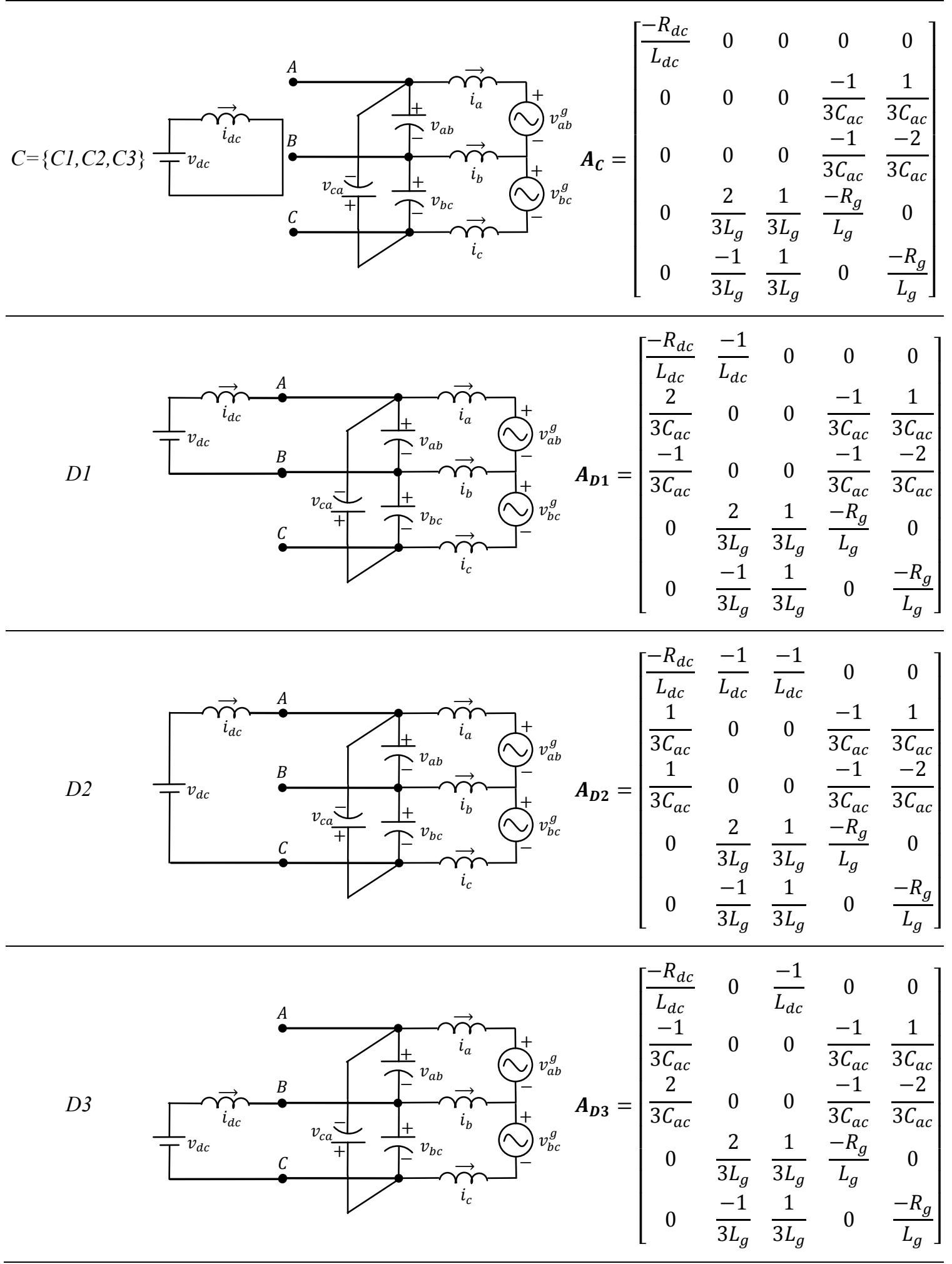


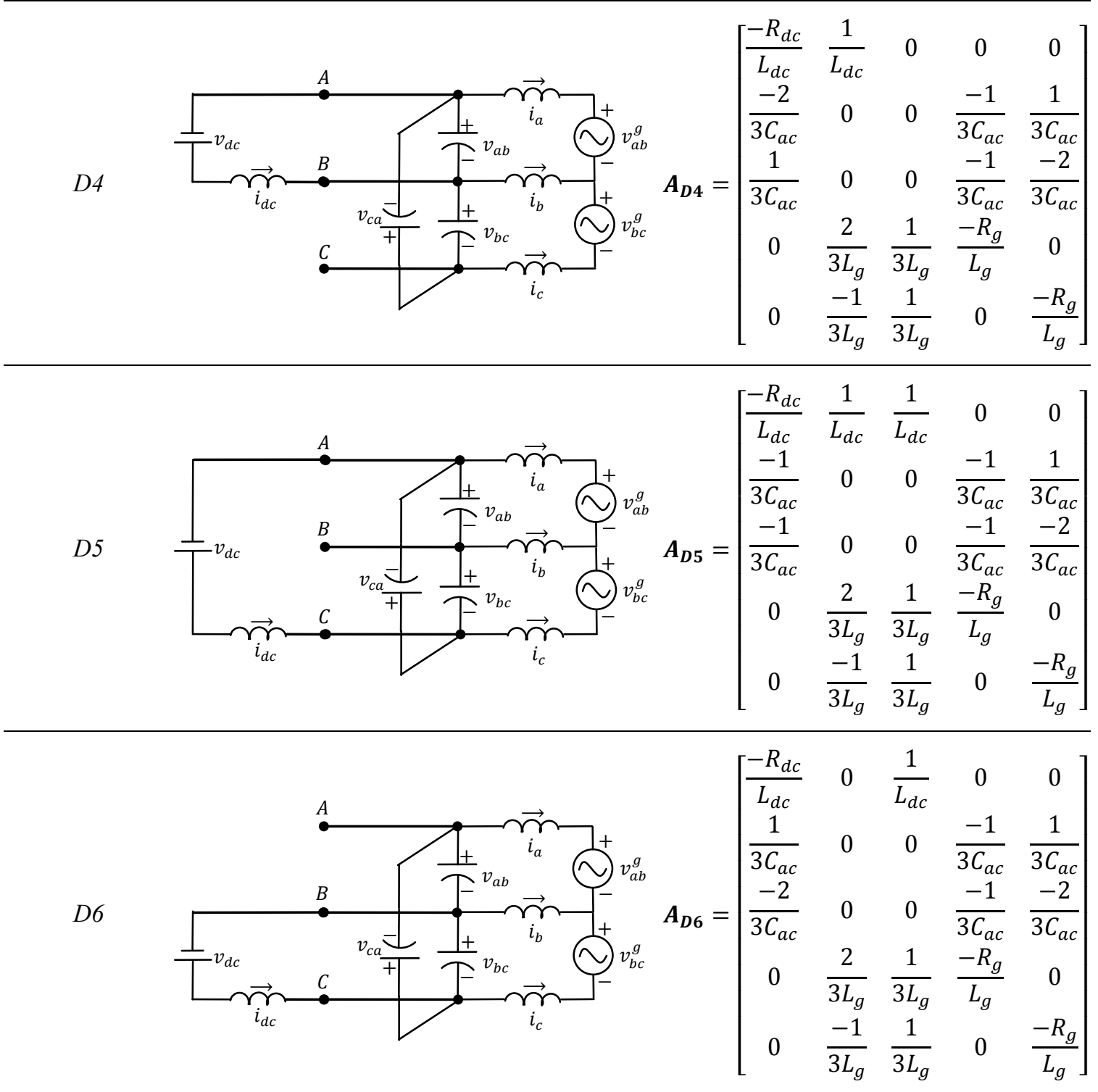

In these state-space representations, the state vector $\boldsymbol{x}$ consists of the state variables of the system which are the independent inductor current and capacitor voltages, i.e. $\boldsymbol{x}=\left[i_{d c}, v_{a b}, v_{b c}, i_{a}, i_{b}\right]^{T}$, where the superscript $T$ denotes the matrix transpose. 
The dc-source voltage, $v_{d c}$, and the grid voltages, $v_{a b}^{g}$ and $v_{b c}^{g}$, are the only inputs to all circuits, i.e.

$$
\boldsymbol{u}_{C}=\boldsymbol{u}_{\boldsymbol{D} 1}=\boldsymbol{u}_{\boldsymbol{D} 2}=\cdots=\boldsymbol{u}_{\boldsymbol{D} 6}=\boldsymbol{u}=\left[v_{d c}, v_{a b}^{g}, v_{b c}^{g}\right]^{T},
$$

and all states have the same control matrix,

$$
\boldsymbol{B}_{\boldsymbol{C}}=\boldsymbol{B}_{\boldsymbol{D} 1}=\boldsymbol{B}_{\boldsymbol{D} 2}=\cdots=\boldsymbol{B}_{\boldsymbol{D} 6}=\boldsymbol{B}=\left[\begin{array}{ccc}
\frac{1}{L_{d c}} & 0 & 0 \\
0 & 0 & 0 \\
0 & 0 & 0 \\
0 & \frac{-2}{3 L_{g}} & \frac{-1}{3 L_{g}} \\
0 & \frac{1}{3 L_{g}} & \frac{-1}{3 L_{g}}
\end{array}\right] .
$$

\subsection{State-Space-Averaged Model of the SSBI}

In this chapter, the state-space averaging method, is applied to the grid-connected three-phase SSBI in order to approximate its time-varying state-pace model by a timeinvariant one. If one can put the time-varying differential equations of the single-stage boost inverter in the standard form of (3.12) and show the periodicity and continuity of the associated vector function over a switching period, i.e. $T_{s}$, then it is possible to apply the theorem of periodic averaging (see Appendix A), and approximate the system model with a set of time-invariant differential equations. Throughout the following, it is assumed that the switching operation is ideal and the switches are lossless and operated at sufficiently high frequency so that the switching action itself does not affect the evolution of the states. As a result, the SSBI operates in continuous mode and the state-space averaging can be applied [49, 57, 59]. 


\subsubsection{Large-Signal Model}

As the first step, the averaging technique is applied to the state-space equations of the grid-connected SSBI system in Sector (I). According to Table 6.1, and the proposed switching pattern in Chapter 4, the state space equation of the grid-connected SSBI in Sector (I) can be written as follows:

$\dot{x}=A_{I}(t) x+B u$
$y=C x+D u$

where, the input vector, $\boldsymbol{u}$, and control matrix, $\boldsymbol{B}$, have been defined in (6.1) and (6.2). (The output matrices $\boldsymbol{C}$ and $\boldsymbol{D}$ can be arbitrarily defined based on the desired outputs of the system, which are discussed here) The system matrix for Sector (I), $\boldsymbol{A}_{\boldsymbol{I}}(t)$, varies with time as:

$\boldsymbol{A}_{\boldsymbol{I}}(t)=\left\{\begin{array}{ll}\boldsymbol{A}_{\boldsymbol{C}} ; & n T_{s} \leq t<n T_{s}+t_{c} \\ \boldsymbol{A}_{\boldsymbol{D} 1} ; & n T_{s}+t_{c} \leq t<n T_{s}+t_{c}+t_{d 1} \\ \boldsymbol{A}_{\boldsymbol{D} 2} ; & n T_{s}+t_{c}+t_{d 1} \leq t<(n+1) T_{s}\end{array} \quad\right.$ for $\quad n=\{1,2,3, \ldots\}$

where, $T_{s}$ is the switching cycle, $t_{c}$ is the charging time-interval, and $t_{d 1}$, and $t_{d 2}$, are the discharging time-intervals. The system matrices in charging and discharging states $C, D 1$, and $D 2$, have been provided in Table 6.1, where:

$$
\boldsymbol{A}_{\boldsymbol{C}}=\left[\begin{array}{ccccc}
\frac{-R_{d c}}{L_{d c}} & 0 & 0 & 0 & 0 \\
0 & 0 & 0 & \frac{-1}{3 C_{a c}} & \frac{1}{3 C_{a c}} \\
0 & 0 & 0 & \frac{-1}{3 C_{a c}} & \frac{-2}{3 C_{a c}} \\
0 & \frac{2}{3 L_{g}} & \frac{1}{3 L_{g}} & \frac{-R_{g}}{L_{g}} & 0 \\
0 & \frac{-1}{3 L_{g}} & \frac{1}{3 L_{g}} & 0 & \frac{-R_{g}}{L_{g}}
\end{array}\right]
$$




$$
\begin{gathered}
\boldsymbol{A}_{\boldsymbol{D} \mathbf{1}}=\left[\begin{array}{ccccc}
\frac{-R_{d c}}{L_{d c}} & \frac{-1}{L_{d c}} & 0 & 0 & 0 \\
\frac{2}{3 C_{a c}} & 0 & 0 & \frac{-1}{3 C_{a c}} & \frac{1}{3 C_{a c}} \\
\frac{-1}{3 C_{a c}} & 0 & 0 & \frac{-1}{3 C_{a c}} & \frac{-2}{3 C_{a c}} \\
0 & \frac{2}{3 L_{g}} & \frac{1}{3 L_{g}} & \frac{-R_{g}}{L_{g}} & 0 \\
0 & \frac{-1}{3 L_{g}} & \frac{1}{3 L_{g}} & 0 & \frac{-R_{g}}{L_{g}}
\end{array}\right] \\
\boldsymbol{A}_{\boldsymbol{D 2}}=\left[\begin{array}{ccccc}
\frac{-R_{d c}}{L_{d c}} & \frac{-1}{L_{d c}} & \frac{-1}{L_{d c}} & 0 & 0 \\
\frac{1}{3 C_{a c}} & 0 & 0 & \frac{-1}{3 C_{a c}} & \frac{1}{3 C_{a c}} \\
\frac{1}{3 C_{a c}} & 0 & 0 & \frac{-1}{3 C_{a c}} & \frac{-2}{3 C_{a c}} \\
0 & \frac{2}{3 L_{g}} & \frac{1}{3 L_{g}} & \frac{-R_{g}}{L_{g}} & 0 \\
0 & \frac{-1}{3 L_{g}} & \frac{1}{3 L_{g}} & 0 & \frac{-R_{g}}{L_{g}}
\end{array}\right]
\end{gathered}
$$

As can be seen in (6.4), the system matrix of the system in Sector (I), $\boldsymbol{A}_{\boldsymbol{I}}(t)$, is a function of time, however, by applying the theorem of periodic averaging [29], the system can be approximated by the following state-space equation:

$\dot{\tilde{x}}=\bar{A}_{I} \tilde{\boldsymbol{x}}+\bar{B} \boldsymbol{u}$

where, $\widetilde{\boldsymbol{x}}=\left[\tilde{\boldsymbol{i}}_{d c}, \tilde{v}_{a b}, \tilde{v}_{b c}, \tilde{\boldsymbol{i}}_{a}, \tilde{i}_{b}\right]^{T}$ is the approximate state vector, and the matrix $\overline{\boldsymbol{A}}_{\boldsymbol{I}}$ is the averaged system matrix for Sector (I),

$$
\overline{\boldsymbol{A}}_{\boldsymbol{I}}=\left[d_{c} \boldsymbol{A}_{\boldsymbol{C}}+d_{1} \boldsymbol{A}_{\boldsymbol{D} \mathbf{1}}+d_{2} \boldsymbol{A}_{\boldsymbol{D} 2}\right]=\left[\begin{array}{ccccc}
\frac{-R_{d c}}{L_{d c}} & \frac{-\left(d_{1}+d_{2}\right)}{L_{d c}} & \frac{-d_{2}}{L_{d c}} & 0 & 0 \\
\frac{2 d_{1}+d_{2}}{3 C_{a c}} & 0 & 0 & \frac{-1}{3 C_{a c}} & \frac{1}{3 C_{a c}} \\
\frac{-d_{1}+d_{2}}{3 C_{a c}} & 0 & 0 & \frac{-1}{3 C_{a c}} & \frac{-2}{3 C_{a c}} \\
0 & \frac{2}{3 L_{g}} & \frac{1}{3 L_{g}} & \frac{-R_{g}}{L_{g}} & 0 \\
0 & \frac{-1}{3 L_{g}} & \frac{1}{3 L_{g}} & 0 & \frac{-R_{g}}{L_{g}}
\end{array}\right]
$$

where, $d_{c}=t_{c} / T_{s}, d_{1}=t_{d 1} / T_{s}$, and $d_{2}=t_{d 2} / T_{s}$, are the corresponding duty ratios. It should be also noted that $\overline{\boldsymbol{B}}=\boldsymbol{B}$. 
Table 6.2: Averaged system matrices of the grid-connected SSBI system for all sectors

\begin{tabular}{|c|c|c|c|c|c|c|c|}
\hline Sector & Averag & ge System 1 & Matrix & & & & \\
\hline I & $\bar{A}_{I}=\left[d_{c} A_{C}+d_{1} A_{D 1}+d_{2} A_{D 2}\right]=$ & $\begin{array}{c}\frac{-R_{d c}}{L_{d c}} \\
\frac{2 d_{1}+d_{2}}{3 C_{a c}} \\
\frac{-d_{1}+d_{2}}{3 C_{a c}} \\
0 \\
0\end{array}$ & $\frac{-\left(d_{1}\right.}{L}$ & $\frac{\left.+d_{2}\right)}{d c}$ & $\begin{array}{c}\frac{-d_{2}}{L_{d c}} \\
0 \\
0 \\
\frac{1}{3 L_{g}} \\
\frac{1}{3 L_{g}}\end{array}$ & $\begin{array}{c}0 \\
\frac{-1}{3 C_{a c}} \\
\frac{-1}{3 C_{a c}} \\
\frac{-R_{g}}{L_{g}} \\
0\end{array}$ & $\begin{array}{c}0 \\
\frac{1}{3 C_{a c}} \\
\frac{-2}{3 C_{a c}} \\
0 \\
\frac{-R_{g}}{L_{g}}\end{array}$ \\
\hline II & $\bar{A}_{I I}=\left[d_{c} A_{C}+d_{1} A_{D 2}+d_{2} A_{D 3}\right]=$ & {$\left[\begin{array}{c}\frac{-R_{d c}}{L_{d c}} \\
\frac{d_{1}-d_{2}}{3 C_{a c}} \\
\frac{d_{1}+2 d_{2}}{3 C_{a c}} \\
0 \\
0\end{array}\right.$} & $\begin{array}{c}0 \\
\frac{2}{3 L_{g}} \\
\frac{-1}{3 L_{g}}\end{array}$ & $\frac{-\left(d_{1}\right.}{L}$ & $\begin{array}{l}\left.+d_{2}\right) \\
d c \\
0\end{array}$ & $\begin{array}{c}0 \\
\frac{-1}{3 C_{a c}} \\
\frac{-1}{3 C_{a c}} \\
\frac{-R_{g}}{L_{g}} \\
0\end{array}$ & $\begin{array}{c}0 \\
\frac{1}{3 C_{a c}} \\
\frac{-2}{3 C_{a c}} \\
0 \\
\frac{-R_{g}}{L_{g}}\end{array}$ \\
\hline III & $\bar{A}_{I I I}=\left[d_{c} \boldsymbol{A}_{C}+d_{1} \boldsymbol{A}_{D 3}+d_{2} \boldsymbol{A}_{D 4}\right]=$ & $=\left[\begin{array}{r}\frac{-R_{c}}{L_{d}} \\
-\left(d_{1}+\right. \\
3 C_{a} \\
\frac{2 d_{1}+}{3 C_{a}} \\
0\end{array}\right.$ & $\begin{array}{l}\frac{c}{\left.2 d_{2}\right)} \\
\frac{d_{2}}{c}\end{array}$ & $\begin{array}{c}\frac{d_{2}}{L_{d c}} \\
0 \\
0 \\
\frac{2}{3 L_{g}} \\
\frac{-1}{3 L_{g}}\end{array}$ & $\begin{array}{c}\frac{-d_{1}}{L_{d c}} \\
0 \\
0 \\
\frac{1}{3 L_{g}} \\
\frac{1}{3 L_{g}}\end{array}$ & $\begin{array}{c}0 \\
\frac{-1}{3 C_{a c}} \\
\frac{-1}{3 C_{a c}} \\
\frac{-R_{g}}{L_{g}} \\
0\end{array}$ & $\left.\begin{array}{c}0 \\
\frac{1}{3 C_{a c}} \\
\frac{-2}{3 C_{a c}} \\
0 \\
\frac{-R_{g}}{L_{g}}\end{array}\right]$ \\
\hline
\end{tabular}




\begin{tabular}{|c|c|c|c|c|c|}
\hline IV & $\overline{\boldsymbol{A}}_{I V}=\left[d_{c} \boldsymbol{A}_{\boldsymbol{C}}+d_{1} A_{D 4}+d_{2} \boldsymbol{A}_{D 5}\right]=$ & {$\left[\begin{array}{c}\frac{-R_{d c}}{L_{d c}} \\
\frac{-\left(2 d_{1}+d_{2}\right)}{3 C_{a c}} \\
\frac{d_{1}-d_{2}}{3 C_{a c}} \\
0 \\
0\end{array}\right.$} & $\begin{array}{c}\frac{\left(d_{1}+d_{2}\right)}{L_{d c}} \\
0 \\
0 \\
\frac{2}{3 L_{g}} \\
\frac{-1}{3 L_{g}}\end{array}$ & $\begin{array}{cc}\frac{d_{2}}{L_{d c}} & 0 \\
0 & \frac{-1}{3 C_{a}} \\
0 & \frac{-1}{3 C_{a}} \\
\frac{1}{3 L_{g}} & \frac{-R_{c}}{L_{g}} \\
\frac{1}{3 L_{g}} & 0\end{array}$ & $\begin{array}{cc} & 0 \\
\frac{1}{a c} & \frac{1}{3 C_{a c}} \\
\frac{1}{a c} & \frac{-2}{3 C_{a c}} \\
\frac{2_{g}}{g} & 0 \\
& \frac{-R_{g}}{L_{g}}\end{array}$ \\
\hline V & $\overline{\boldsymbol{A}}_{\boldsymbol{V}}=\left[d_{c} \boldsymbol{A}_{C}+d_{1} \boldsymbol{A}_{D 5}+d_{2} \boldsymbol{A}_{D 6}\right]=$ & $\begin{array}{c}\frac{-R_{d c}}{L_{d c}} \\
\frac{-d_{1}+d_{2}}{3 C_{a c}} \\
\frac{-\left(d_{1}+2 d_{2}\right)}{3 C_{a c}} \\
0 \\
0\end{array}$ & $\begin{array}{cc}\frac{d_{1}}{L_{d c}} & \frac{\left(d_{1}+\right.}{L_{d c}} \\
0 & 0 \\
0 & 0 \\
\frac{2}{3 L_{g}} & \frac{1}{3 L_{c}} \\
\frac{-1}{3 L_{g}} & \frac{1}{3 L_{c}}\end{array}$ & $\begin{array}{c}0 \\
\frac{-1}{3 C_{a c}} \\
\frac{-1}{3 C_{a c}} \\
\frac{-R_{g}}{L_{g}} \\
0\end{array}$ & $\begin{array}{cc} & 0 \\
\frac{1}{a c} & \frac{1}{3 C_{a c}} \\
\frac{1}{a c} & \frac{-2}{3 C_{a c}} \\
\underline{g} & 0 \\
\frac{-R_{g}}{L_{g}}\end{array}$ \\
\hline VI & $\overline{\boldsymbol{A}}_{V I}=\left[d_{c} \boldsymbol{A}_{C}+d_{1} \boldsymbol{A}_{D 6}+d_{2} \boldsymbol{A}_{D 1}\right]=$ & $=\left[\begin{array}{c}\frac{-R_{d c}}{L_{d c}} \\
\frac{d_{1}+2 d_{2}}{3 C_{a c}} \\
\frac{-\left(2 d_{1}+d_{2}\right)}{3 C_{a c}} \\
0 \\
0\end{array}\right.$ & $\begin{array}{c}\frac{-d_{2}}{L_{d c}} \\
0 \\
0 \\
\frac{2}{3 L_{g}} \\
\frac{-1}{3 L_{g}}\end{array}$ & $\begin{array}{c}0 \\
\frac{-1}{3 C_{a c}} \\
\frac{-1}{3 C_{a c}} \\
\frac{-R_{g}}{L_{g}} \\
0\end{array}$ & $\left.\begin{array}{c}0 \\
\frac{1}{3 C_{a c}} \\
\frac{-2}{3 C_{a c}} \\
0 \\
\frac{-R_{g}}{L_{g}}\end{array}\right]$ \\
\hline
\end{tabular}

The obtained system matrix in (6.9) is time-varying, because it is a function of discharging duty ratios, $d_{1}$ and $d_{2}$, which are varying with time (see (5.7) and (5.8)). However, in comparison with the fast variations of the system during a switching cycle, they can be considered as relatively slowly-varying terms. 
The averaged system matrices for other sectors, $\bar{A}_{I I}, \bar{A}_{I I I}, \bar{A}_{I V}, \bar{A}_{V}, \bar{A}_{V I}$, are provided in Table 6.2. It might be interesting to know that all of the averaged system matrices are similar, because they all have the same eigenvalues [69].

As can be seen in Table 6.2, for the six sectors of the proposed phaseor pulse-withmodulation (PPWM) switching pattern, there are six different sets of equations, and consequently six distinct system matrices, namely $\bar{A}_{I}, \bar{A}_{I I}, \bar{A}_{I I I}, \bar{A}_{I V}, \bar{A}_{V}, \bar{A}_{V I}$. This means that, the period of the SSBI equations is equal to the line voltage cycle, e.g. $60 \mathrm{~Hz}$. Moreover, these matrices are time-variant, as $d_{1}$ and $d_{2}$ vary by time. Applying the Park's transformation (see (5.9) and (5.10)) to the state-space-averaged of (6.8), considering the system matrix of (6.9) results in:

$\dot{\widetilde{x}}_{d q}=\overline{\boldsymbol{A}}_{d q I} \widetilde{\boldsymbol{x}}_{d q}+\boldsymbol{B}_{d q} \boldsymbol{u}_{d q}$

where,

$$
\begin{aligned}
\tilde{\boldsymbol{x}}_{\boldsymbol{d q}} & =\left[\tilde{\boldsymbol{i}}_{d c}, \tilde{v}_{q}, \tilde{v}_{d}, \tilde{\boldsymbol{i}}_{q}, \tilde{i}_{d}\right]^{T} \\
\boldsymbol{u}_{\boldsymbol{d q}} & =\left[v_{d c}, v_{q}^{g}, v_{d}^{g}\right]^{T} \\
\overline{\boldsymbol{A}}_{\boldsymbol{d q I}} & =\left[\begin{array}{ccccc}
\frac{-R_{d c}}{L_{d c}} & \frac{-\left[d_{1} \cos \left(\theta_{f}\right)+d_{2} \cos \left(\theta_{f}-\frac{\pi}{3}\right)\right]}{L_{d c}} & \frac{-\left[d_{1} \sin \left(\theta_{f}\right)+d_{2} \sin \left(\theta_{f}-\frac{\pi}{3}\right)\right]}{L_{d c}} & 0 & 0 \\
\frac{2\left[d_{1} \cos \left(\theta_{f}\right)+d_{2} \cos \left(\theta_{f}-\frac{\pi}{3}\right)\right]}{3 C_{a c}} & 0 & -\dot{\theta}_{f} & \frac{-1}{2 c_{a c}} & \frac{-\sqrt{3}}{6 c_{a c}} \\
\frac{2\left[d_{1} \sin \left(\theta_{f}\right)+d_{2} \sin \left(\theta_{f}-\frac{\pi}{3}\right)\right]}{3 C_{a c}} & \dot{\theta}_{f} & 0 & \frac{\sqrt{3}}{6 C_{a c}} & \frac{-1}{2 C_{a c}} \\
0 & \frac{1}{2 L_{g}} & \frac{-\sqrt{3}}{6 L_{g}} & \frac{-R_{g}}{L_{g}} & -\dot{\theta}_{f} \\
0 & \frac{\sqrt{3}}{6 L_{g}} & \frac{1}{2 L_{g}} & \dot{\theta}_{f} & \frac{-R_{g}}{L_{g}}
\end{array}\right]
\end{aligned}
$$


$\boldsymbol{B}_{\boldsymbol{d} \boldsymbol{q}}=\left[\begin{array}{ccc}\frac{1}{L_{d c}} & 0 & 0 \\ 0 & 0 & 0 \\ 0 & 0 & 0 \\ 0 & \frac{-1}{2 L_{g}} & \frac{\sqrt{3}}{6 L_{g}} \\ 0 & \frac{-\sqrt{3}}{6 L_{g}} & \frac{-1}{2 L_{g}}\end{array}\right]$

which is the state-space-averaged model of the grid-connected SSBI for Sector (I) in the rotating reference frame $\left(\theta_{f}\right.$ is the angular displacement of the rotating reference frame). The averaged system matrices for the other sectors, $\bar{A}_{\boldsymbol{d q I I}}, \overline{\boldsymbol{A}}_{\boldsymbol{d q I I I}}, \ldots, \overline{\boldsymbol{A}}_{\boldsymbol{d q V I}}$ (see Table 6.3), can be obtained in the same way, while the input vector, $\boldsymbol{u}_{\boldsymbol{d q}}$, and the control matrix, $\boldsymbol{B}_{\boldsymbol{d q}}$, are the same as (6.12) and (6.14) for all sectors. The averaged system matrices are presented in Table 6.3.

Alternatively, Table 6.3, could be obtained by applying (5.9) and (5.10) to the state-space equations of Table 6.1 (see Table 6.4), and then averaging them over a switching cycle. Anyhow, (6.13) is still a function of $d_{1}$ and $d_{2}$. Substituting (5.7) and (5.8) in (6.13), yields the following matrix in the rotating reference frame:

$$
\bar{A}_{d \boldsymbol{~} I}=\left[\begin{array}{ccccc}
\frac{-R_{d c}}{L_{d c}} & \frac{-\sqrt{3} m \cos \left(\theta_{f}-\theta\right)}{2 L_{d c}} & \frac{-\sqrt{3} m \sin \left(\theta_{f}-\theta\right)}{2 L_{d c}} & 0 & 0 \\
\frac{\sqrt{3} m \cos \left(\theta_{f}-\theta\right)}{3 C_{a c}} & 0 & -\dot{\theta}_{f} & \frac{-1}{2 C_{a c}} & \frac{-\sqrt{3}}{6 C_{a c}} \\
\frac{\sqrt{3} m \sin \left(\theta_{f}-\theta\right)}{3 C_{a c}} & \dot{\theta}_{f} & 0 & \frac{\sqrt{3}}{6 C_{a c}} & \frac{-1}{2 C_{a c}} \\
0 & \frac{1}{2 L_{g}} & \frac{-\sqrt{3}}{6 L_{g}} & \frac{-R_{g}}{L_{g}} & -\dot{\theta}_{f} \\
0 & \frac{\sqrt{3}}{6 L_{g}} & \frac{1}{2 L_{g}} & \dot{\theta}_{f} & \frac{-R_{g}}{L_{g}}
\end{array}\right]
$$


Table 6.3: Averaged system matrices of the grid-connected SSBI system in the $d q$-frame of reference

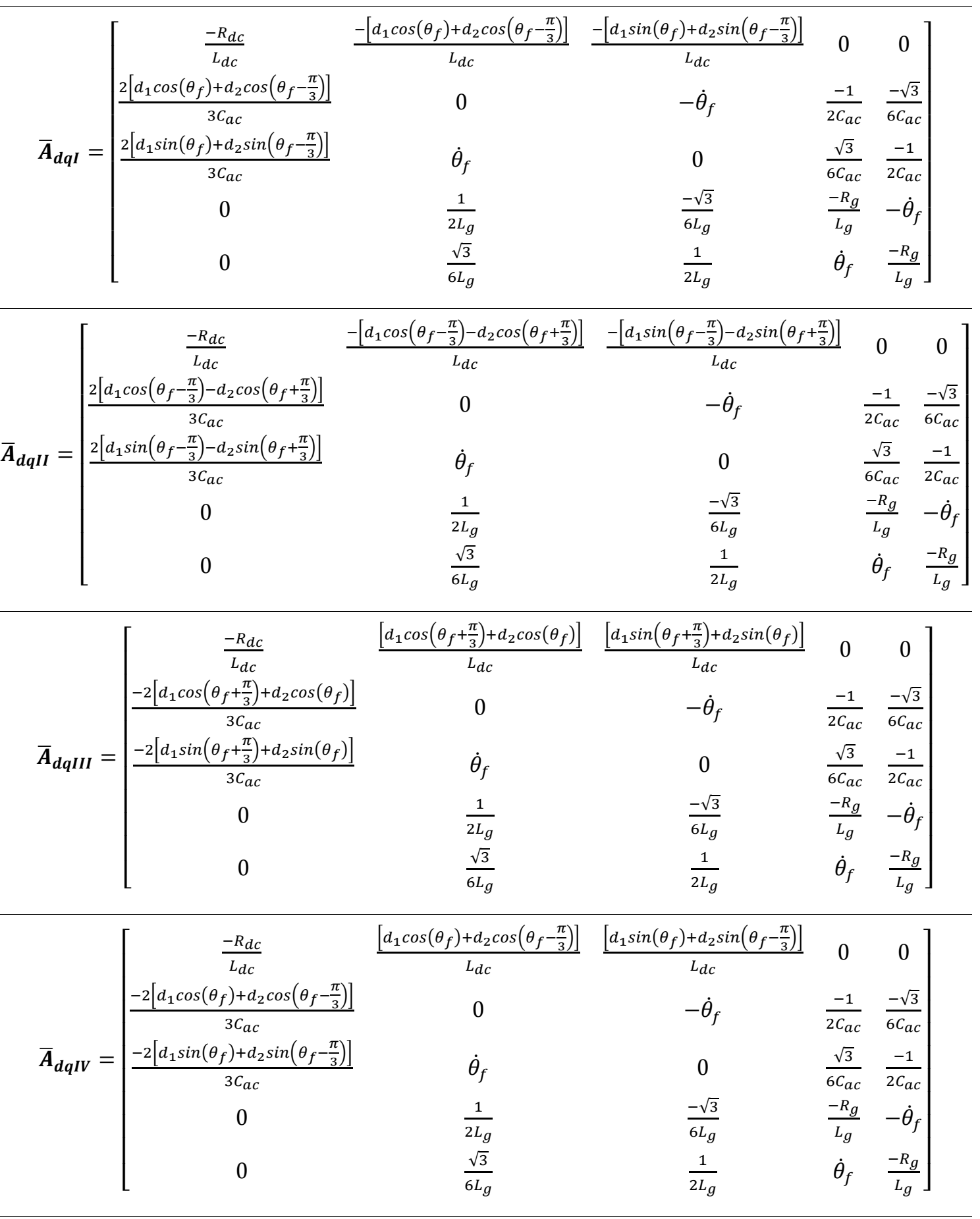


Table 6.3: Continued

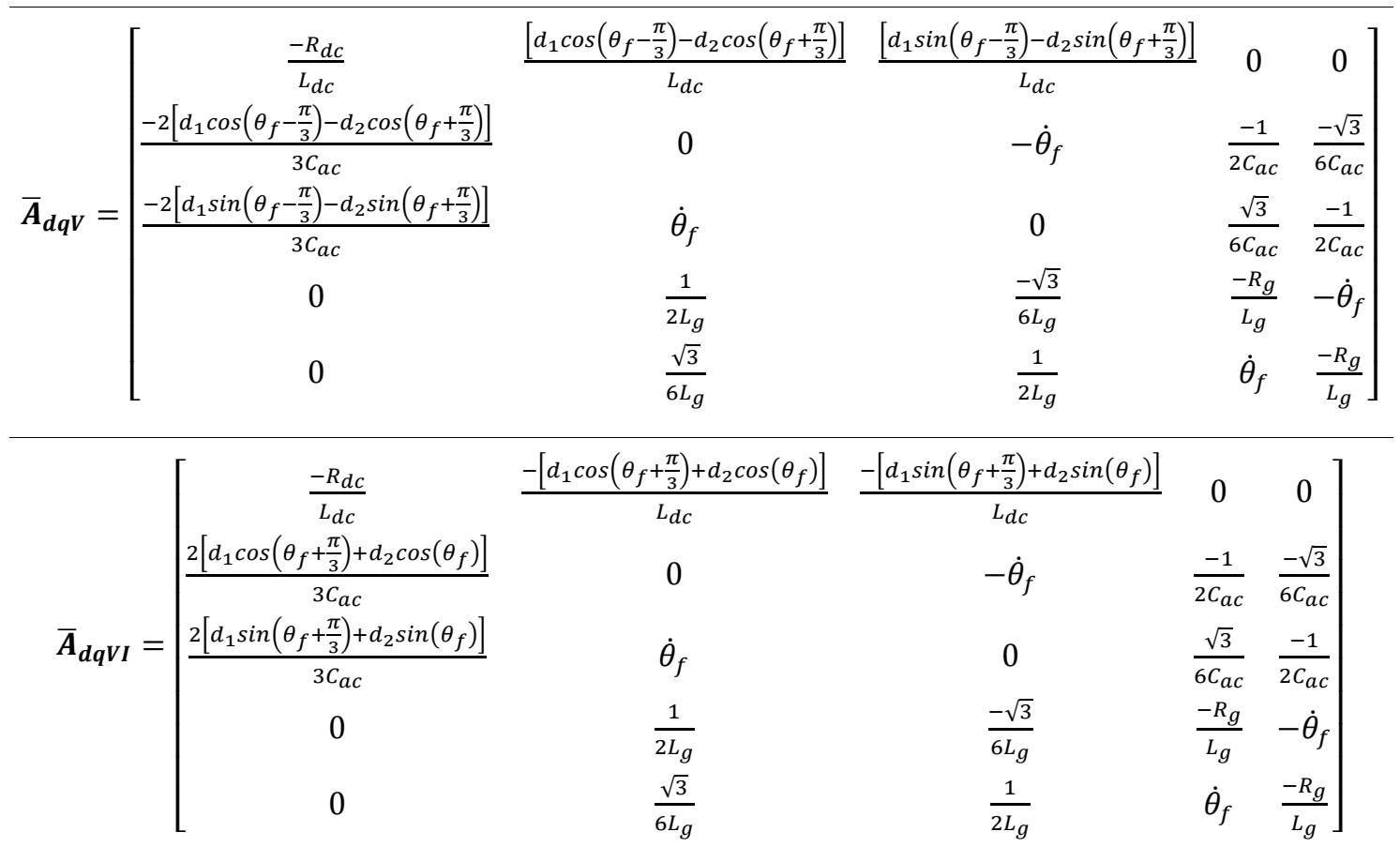

As can be seen, the obtained averaged system matrix, $\overline{\boldsymbol{A}}_{\boldsymbol{d q I}}$, contains three timedependent parameters, i.e. the angular displacement of the reference line voltage, $\theta$, (see (5.7) and (5.8)), as well as the angular displacement and velocity of the rotating frame of reference, $\theta_{f}$ and $\dot{\theta}_{f}$, respectively. In order to get rid of these time-dependent terms, it is sufficient to assume $\dot{\theta}_{f}$ and $\theta_{f}-\theta$ as constants. This can be achieved through:

i) Fixing the rotating frame on the synchronous frame of the grid,

$$
\theta_{f}(t)=\omega_{g} t+\theta_{g}(0)
$$

ii) And,

$$
\theta(t)=\theta_{f}(t)-\phi=\omega_{g} t+\theta_{g}(0)-\phi
$$


Table 6.4: System matrices for the switching states of the grid-connected SSBI in the $d q$-frame of reference

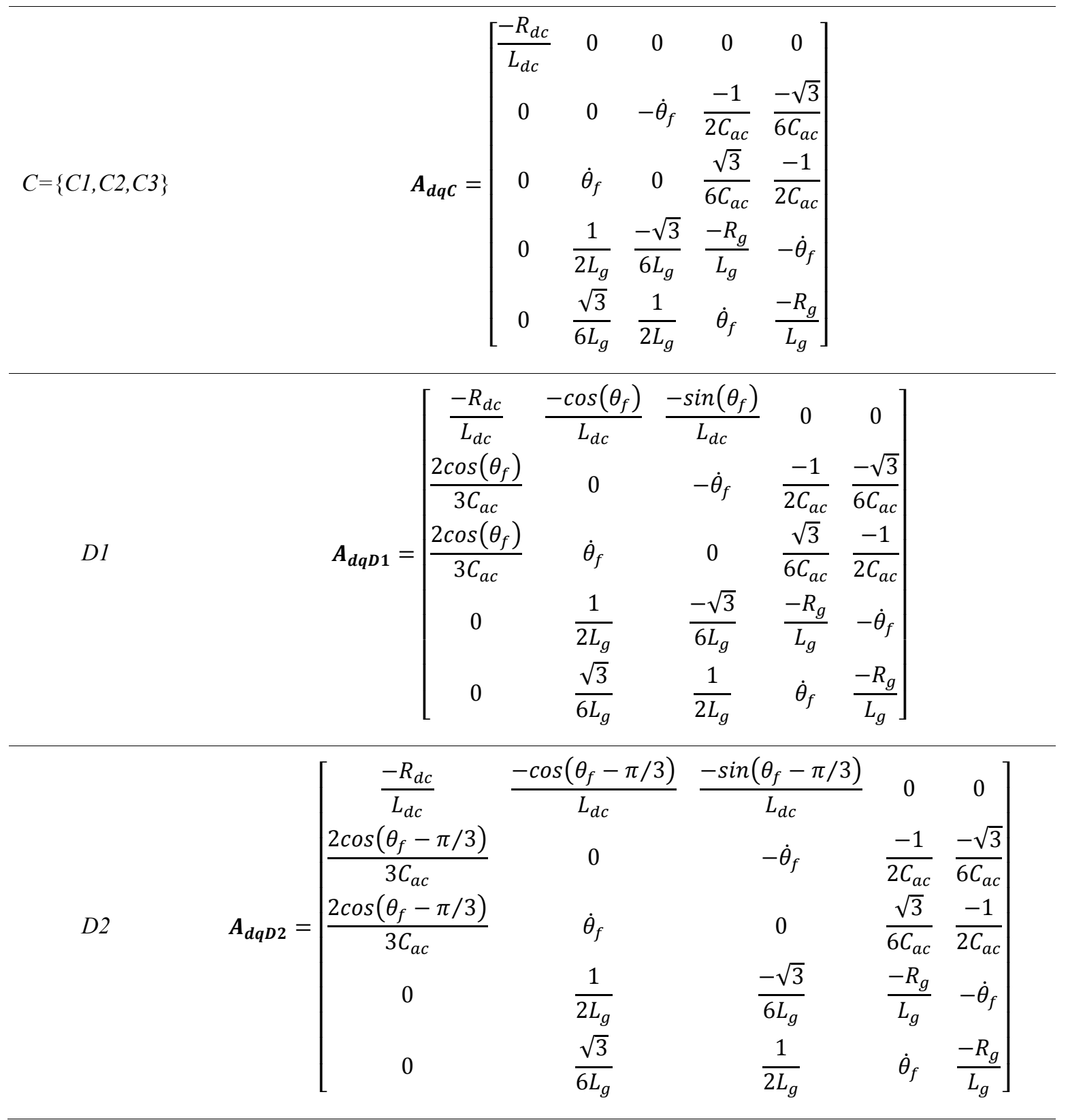


Table 6.4: Continued

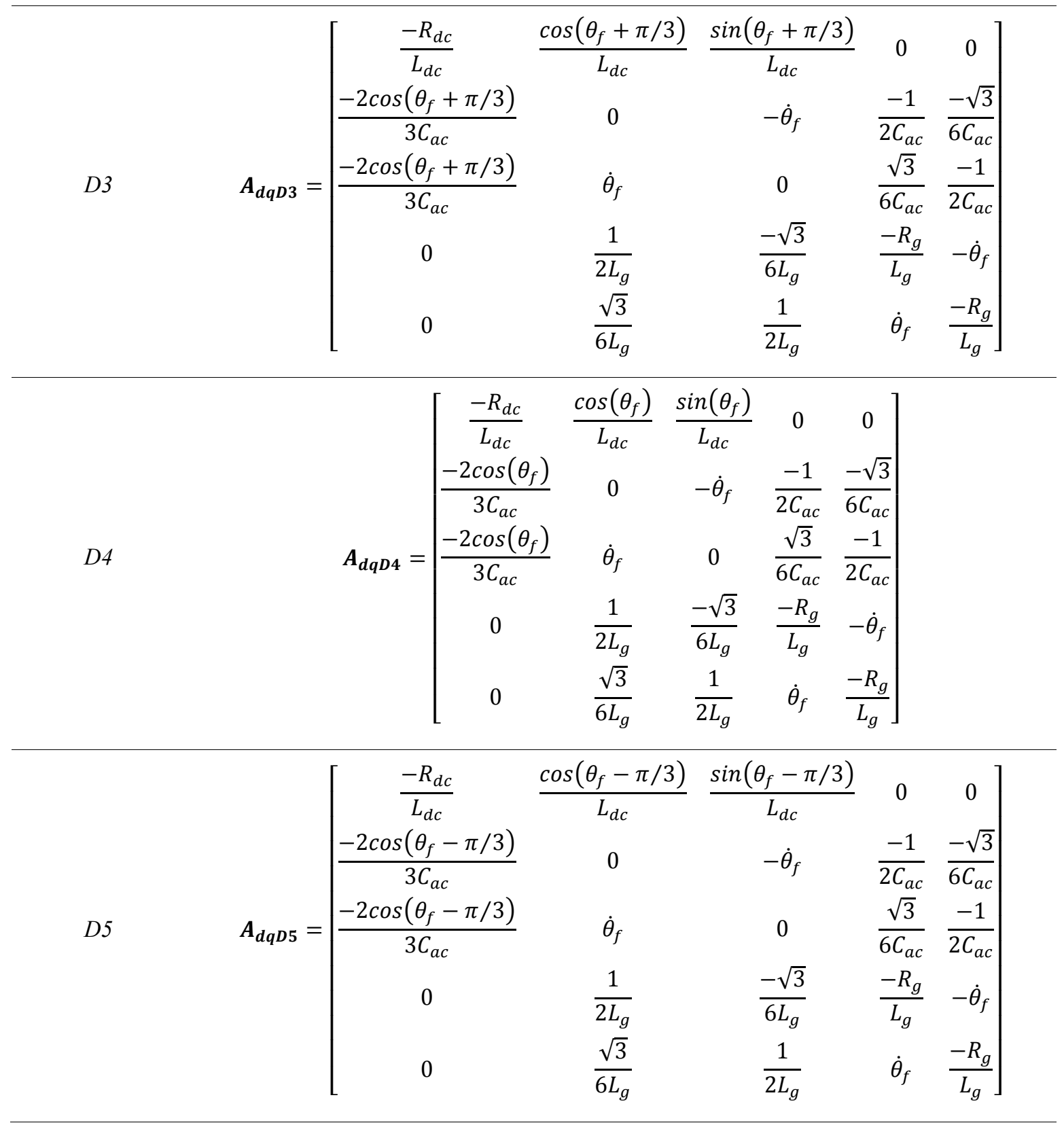




$$
\text { D6 } \quad \boldsymbol{A}_{\boldsymbol{d q D 6} \mathbf{n}}=\left[\begin{array}{ccccc}
\frac{-R_{d c}}{L_{d c}} & \frac{-\cos \left(\theta_{f}+\pi / 3\right)}{L_{d c}} & \frac{-\sin \left(\theta_{f}+\pi / 3\right)}{L_{d c}} & 0 & 0 \\
\frac{2 \cos \left(\theta_{f}+\pi / 3\right)}{3 C_{a c}} & 0 & -\dot{\theta}_{f} & \frac{-1}{2 C_{a c}} & \frac{-\sqrt{3}}{6 C_{a c}} \\
\frac{2 \cos \left(\theta_{f}+\pi / 3\right)}{3 C_{a c}} & \dot{\theta}_{f} & 0 & \frac{\sqrt{3}}{6 C_{a c}} & \frac{-1}{2 C_{a c}} \\
0 & \frac{1}{2 L_{g}} & \frac{-\sqrt{3}}{6 L_{g}} & \frac{-R_{g}}{L_{g}} & -\dot{\theta}_{f} \\
0 & \frac{\sqrt{3}}{6 L_{g}} & \frac{1}{2 L_{g}} & \dot{\theta}_{f} & \frac{-R_{g}}{L_{g}}
\end{array}\right]
$$

where, $\omega_{g}=2 \pi f_{g}$ is the angular frequency of the grid (usually $f_{g}=60 \mathrm{~Hz}$ ), and $\phi$ is a constant angle, from now on called modulation angle, which can be used for adjusting the phase angle between the output voltage of the SSBI, $v_{a b}$, and the grid voltage, $v_{a b}^{g}$. This implies that, $\phi$ may be used as a control parameter for regulating the injected active power to the grid. Substituting (6.16) and (6.17) in (6.13) provides a time-invariant averaged system matrix:

$$
\overline{\boldsymbol{A}}_{\boldsymbol{d q I}}=\left[\begin{array}{ccccc}
\frac{-R_{d c}}{L_{d c}} & \frac{-\sqrt{3} m \cos (\phi)}{2 L_{d c}} & \frac{-\sqrt{3} m \sin (\phi)}{2 L_{d c}} & 0 & 0 \\
\frac{\sqrt{3} m \cos (\phi)}{3 C_{a c}} & 0 & -\omega_{g} & \frac{-1}{2 C_{a c}} & \frac{-\sqrt{3}}{6 C_{a c}} \\
\frac{\sqrt{3} m \sin (\phi)}{3 C_{a c}} & \omega_{g} & 0 & \frac{\sqrt{3}}{6 C_{a c}} & \frac{-1}{2 C_{a c}} \\
0 & \frac{1}{2 L_{g}} & \frac{-\sqrt{3}}{6 L_{g}} & \frac{-R_{g}}{L_{g}} & -\omega_{g} \\
0 & \frac{\sqrt{3}}{6 L_{g}} & \frac{1}{2 L_{g}} & \omega_{g} & \frac{-R_{g}}{L_{g}}
\end{array}\right]
$$

As was expected, the resulting state-space-averaged model of the grid-connected three-phase SSBI system is independent of the averaging period (or the switching cycle), $T_{s}$. It can also be proved that the same average matrix will be obtained for the other sectors. That is, 


$$
\bar{A}_{d q I}=\bar{A}_{d q I I}=\cdots=\bar{A}_{d q V I}=\bar{A}_{d q}
$$

The large-signal state-space-averaged model of the grid-connected SSBI system, can be represented in the synchronous $d q$-frame of reference as follows:

$$
\frac{d}{d t}\left[\begin{array}{c}
\tilde{i}_{d c} \\
\tilde{v}_{q} \\
\tilde{v}_{d} \\
\tilde{i}_{q} \\
\tilde{i}_{d}
\end{array}\right]=\left[\begin{array}{ccccc}
\frac{-R_{d c}}{L_{d c}} & \frac{-\sqrt{3} m \cos (\phi)}{2 L_{d c}} & \frac{-\sqrt{3} m \sin (\phi)}{2 L_{d c}} & 0 & 0 \\
\frac{\sqrt{3} m \cos (\phi)}{3 C_{a c}} & 0 & -\omega_{g} & \frac{-1}{2 C_{a c}} & \frac{-\sqrt{3}}{6 C_{a c}} \\
\frac{\sqrt{3} m \sin (\phi)}{3 C_{a c}} & \omega_{g} & 0 & \frac{\sqrt{3}}{6 C_{a c}} & \frac{-1}{2 C_{a c}} \\
0 & \frac{1}{2 L_{g}} & \frac{-\sqrt{3}}{6 L_{g}} & \frac{-R_{g}}{L_{g}} & -\omega_{g} \\
0 & \frac{\sqrt{3}}{6 L_{g}} & \frac{1}{2 L_{g}} & \omega_{g} & \frac{-R_{g}}{L_{g}}
\end{array}\right]\left[\begin{array}{c}
\tilde{i}_{d c} \\
\tilde{v}_{q} \\
\tilde{v}_{d} \\
\tilde{i}_{q} \\
\tilde{i}_{d}
\end{array}\right]
$$

It should be noted that (6.20) represents a linear time-invariant system assuming that the modulation index and angle, $m$ and $\phi$, are constant.

\subsubsection{Small-Signal Model}

As one can see in $(6.20),\left[v_{d c}, v_{q}^{g}, v_{d}^{g}\right]^{T}$ represents the system inputs, and the modulation index, $m$, and the modulation angle, $\phi$, are assumed constant. In practice, $m$ and $\phi$ can be used to regulate the injected active and reactive powers to the grid, in gridconnected conditions. In this case, (6.20) will not be any longer a linear model, but it can be linearized around any steady state operating point (see Appendix B). Let us define:

$\widetilde{\boldsymbol{x}}_{\boldsymbol{d q}}=\widetilde{\boldsymbol{X}}_{\boldsymbol{d q}}+\boldsymbol{\delta} \widetilde{\boldsymbol{x}}_{\boldsymbol{d q}}, \quad v_{d c}=V_{d c}+\delta v_{d c}, \quad m=M+\delta m, \quad \phi=\Phi+\delta \phi$ 
where, $\tilde{\boldsymbol{x}}_{\boldsymbol{d q}}=\left[\tilde{i}_{d c}, \tilde{v}_{q}, \tilde{v}_{d}, \tilde{i}_{q}, \tilde{i}_{d}\right]^{T}$ is the approximate state vector in the rotating $d q$-frame of reference, the $\delta$ denotes small-signal perturbation, and capital letters indicate the steady-state components. Thus, the linearized (small-signal) model can be represented as follows:

$$
\begin{aligned}
& \frac{d}{d t}\left[\begin{array}{c}
\delta \tilde{i}_{d c} \\
\delta \tilde{v}_{q} \\
\delta \tilde{v}_{d} \\
\delta \tilde{i}_{q} \\
\delta \tilde{i}_{d}
\end{array}\right]=\left[\begin{array}{ccccc}
\frac{-R_{d c}}{L_{d c}} & \frac{-\sqrt{3} M \cos (\Phi)}{2 L_{d c}} & \frac{-\sqrt{3} M \sin (\Phi)}{2 L_{d c}} & 0 & 0 \\
\frac{\sqrt{3} M \cos (\Phi)}{3 C_{a c}} & 0 & -\omega_{g} & \frac{-1}{2 C_{a c}} & \frac{-\sqrt{3}}{6 C_{a c}} \\
\frac{\sqrt{3} M \sin (\Phi)}{3 C_{a c}} & \omega_{g} & 0 & \frac{\sqrt{3}}{6 C_{a c}} & \frac{-1}{2 C_{a c}} \\
0 & \frac{1}{2 L_{g}} & \frac{-\sqrt{3}}{6 L_{g}} & \frac{-R_{g}}{L_{g}} & -\frac{-\omega_{g}}{\delta L_{g}} \\
0 & \frac{\sqrt{3}}{6 L_{g}} & \frac{1}{2 L_{g}} & \omega_{g} & \frac{-R_{g}}{L_{g}}
\end{array}\right]\left[\begin{array}{l}
\delta \tilde{i}_{d c} \\
\delta \tilde{v}_{q} \\
\delta \tilde{v}_{d} \\
\delta \tilde{i}_{q} \\
\delta \tilde{i}_{d}
\end{array}\right] \\
& +\left[\begin{array}{ccccc}
\frac{1}{L_{d c}} & 0 & 0 & \frac{\sqrt{3}}{2 L_{d c}}\left[-\cos (\Phi) \tilde{V}_{q}+\sin (\Phi) \tilde{V}_{d}\right] & \frac{\sqrt{3} M}{2 L_{d c} T_{S}}\left[\sin (\Phi) \tilde{V}_{q}+\cos (\Phi) \tilde{V}_{d}\right] \\
0 & 0 & 0 & \frac{\sqrt{3} \cos (\Phi) \tilde{I}_{d c}}{3 C_{a c}} & \frac{-\sqrt{3} M \sin (\Phi) \tilde{I}_{d c}}{3 C_{a c}} \\
0 & 0 & 0 & \frac{-\sqrt{3} \sin (\Phi) \tilde{I}_{d c}}{3 C_{a c}} & \frac{-\sqrt{3} M \cos (\Phi) \tilde{I}_{d c}}{3 C_{a c}} \\
0 & \frac{-1}{2 L_{g}} & \frac{\sqrt{3}}{6 L_{g}} & 0 & 0 \\
0 & \frac{-\sqrt{3}}{6 L_{g}} & \frac{-1}{2 L_{g}} & 0 & 0
\end{array}\right]\left[\begin{array}{l}
\delta v_{d c} \\
\delta v_{q}^{g} \\
\delta v_{d}^{g} \\
\delta m \\
\delta \phi
\end{array}\right]
\end{aligned}
$$

As will be seen in the next section, equation (6.22) can be used for investigation of the stability of the grid-connect SSBI system.

\subsection{Eigenvalue Sensitivity Analysis}

In this section, the eigenvalue sensitivities with respect to the system parameters are studied using the developed small-signal model of the SSBI. Herein, the state-spaceaveraged equation (6.22) is used for calculating the eigenvalues of the system. The study is based on the experimental set-up that is built in the laboratory for test and verification. The parameters of the developed set-up are summarized in Table 6.5. It should be noted that the dc-link resistance includes the series resistance of the dc-link inductor and the 
forward bias resistances of the switches and diodes, which will be discussed in simulation and experimental results. Also, the grid voltage frequency is assumed constant, i.e. $\omega_{g}=120 \pi \mathrm{rad} / \mathrm{sec}$. Substituting these values into (6.20) results in the equilibrium point state vector of $\widetilde{X}_{\boldsymbol{d} q}=[5.21,167.2,-7.932,0.2818,0.7446]^{T}$, which corresponds to a line-to-line rms voltage of $120 \mathrm{~V}$ and a line rms current of $0.56 \mathrm{~A}$. This operating point corresponds to about $100 \mathrm{~W}$, which is within the operational range of a typical PV panel. The eigenvalues of the system at this operating point are obtained as $\lambda_{1}=-74.248$, $\lambda_{2,3}=-54.272 \pm j 1389.1$, and $\lambda_{4,5}=-54.142 \pm j 664.99$. Unlike for the stand-alone case, it can be observed that the grid-connected SSBI system does not have any nondominant eigenvalues, and real parts of all the eigenvalues are within the same range. Thus, the dynamic behavior of the system is dominated by all five eigenvalues, which are located in the left-half $s$-plane. Therefore, the system is stable for the parameter values given in Table 6.5. However, it does not necessarily mean that the system will be stable if the system parameters change [79]. Hence, in order to investigate the stability of the system, it is required to perform the sensitivity analysis of the system's eigenvalues to the variations of the parameters. The definition of the sensitivity has been given in Chapter 5 with (5.17) and (5.18).

\section{A. Sensitivity to the DC-Source, $v_{d c}$}

As can be seen in (6.22), the state matrix is independent of $V_{d c}$, therefore, the eigenvalues of the system do not move with the variations of the dc-source voltage. Thus, the dc-source voltage does not affect the stability of the grid-connected three-phase SSBI. This is same as that was observed in Chapter 5, for the stand-alone SSBI system. 
Table 6.5: System parameters

\begin{tabular}{cc}
\hline Parameter & Nominal values \\
\hline$v_{d c}$ & $20 \mathrm{~V}$ \\
\hline$v_{g}$ & $120 \mathrm{Vrms}$ \\
\hline$L_{d c}$ & $10 \mathrm{mH}$ \\
\hline$R_{d c}$ & $0.68 \Omega$ \\
\hline$C_{a c}$ & $13.3 \mu \mathrm{F}$ \\
\hline$L_{g}$ & $26 \mathrm{mH}$ \\
\hline$R_{g}$ & $2.9 \Omega$ \\
\hline$M$ & 0.21 \\
\hline$\Phi$ & $60^{\circ}$ \\
\hline
\end{tabular}

B. Sensitivity to the DC-Link Inductor, $R_{d c}$ and $L_{d c}$

The impacts of $R_{d c}$ and $L_{d c}$ on the eigenvalues of the system are shown in Figures 6.2 and 6.3, respectively. The arrows point in the directions of displacements of the eigenvalues as the associated parameter increases. In these figures, the bold cross signs $(+)$ indicate the location of the eigenvalues for the nominal values of the system's parameters in Table 6.5. In addition to the locations of the eigenvalues, the real part sensitivities of $\lambda_{1}, \lambda_{2}$, and $\lambda_{4}$ and the imaginary part sensitivities of $\lambda_{2}$, and $\lambda_{4}$ are plotted in the lower part of each figure (see also (5.19)).

As can be seen in Figure 6.2, an increase in the resistance of the dc-link moves the eigenvalues of the system to the left, which increases the damping and speed of the system. But, it should be mentioned that any increase in the system's resistance results in increase in the losses, which is not desirable in power applications. 

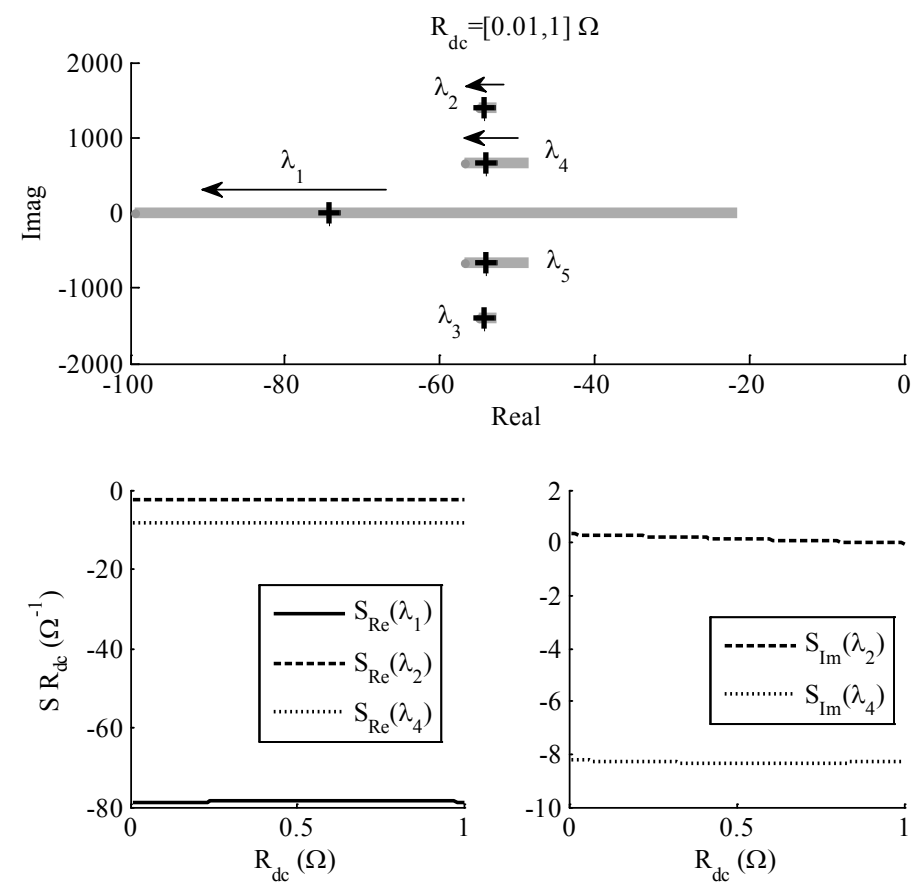

Figure 6.2: Locations of the grid-connected SSBI eigenvalues and their sensitivities versus $R_{d c}$ when it varies between 0.01 and $1 \Omega$

Similarly, it can be seen in Figure 6.3 that as the inductance value of the dc-link inductor increases, $\lambda_{1}, \lambda_{4}$ and $\lambda_{5}$ move toward the imaginary axis, which can slow down the system response. As one can observe from Figures 6.2 and 6.3, the impacts of the dclink parameters, $R_{d c}$ and $L_{d c}$, on the eigenvalue, $\lambda_{2}$ and $\lambda_{3}$, are almost negligible. 

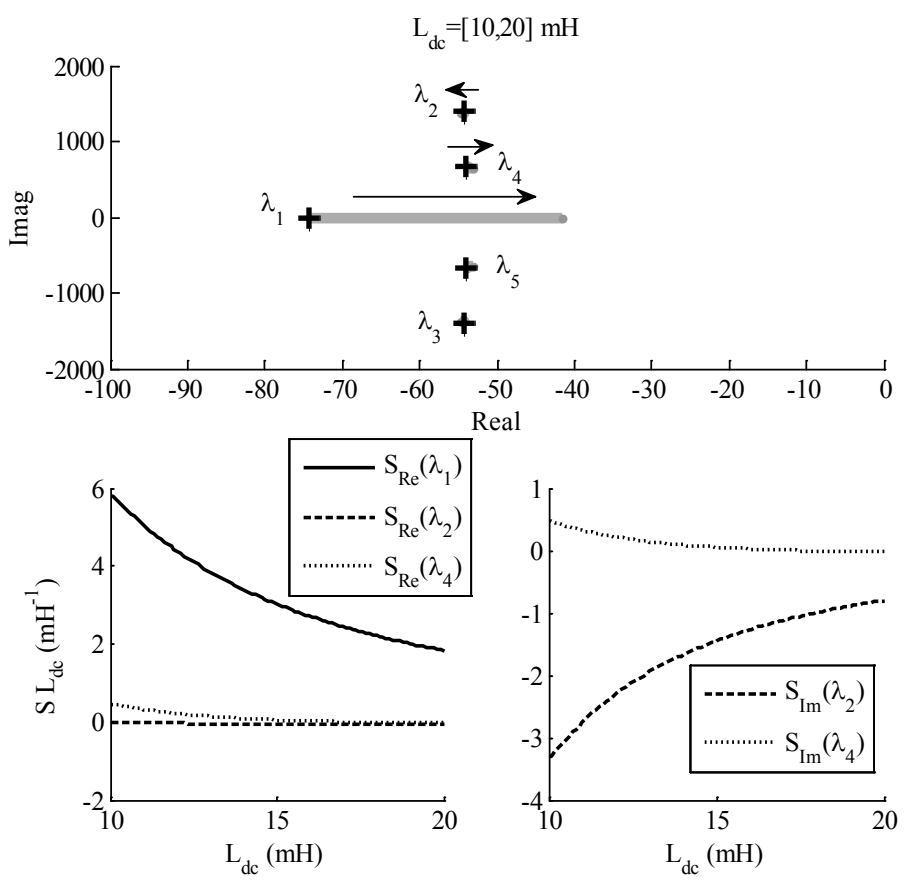

Figure 6.3: Locations of the grid-connected SSBI eigenvalues and their sensitivities versus $L_{d c}$ when it varies between 10 and $20 \mathrm{mH}$

\section{Sensitivity to the AC Capacitors, $C_{a c}$}

The location of the system eigenvalues and their sensitivities to the ac-side capacitance, $C_{a c}$, are shown in Figure 6.4 , where $\lambda_{2}, \lambda_{3}, \lambda_{4}$, and $\lambda_{5}$ move toward the real axis as the capacitance value increases. This reduces the system natural frequencies and increases the damping factor of the system. 

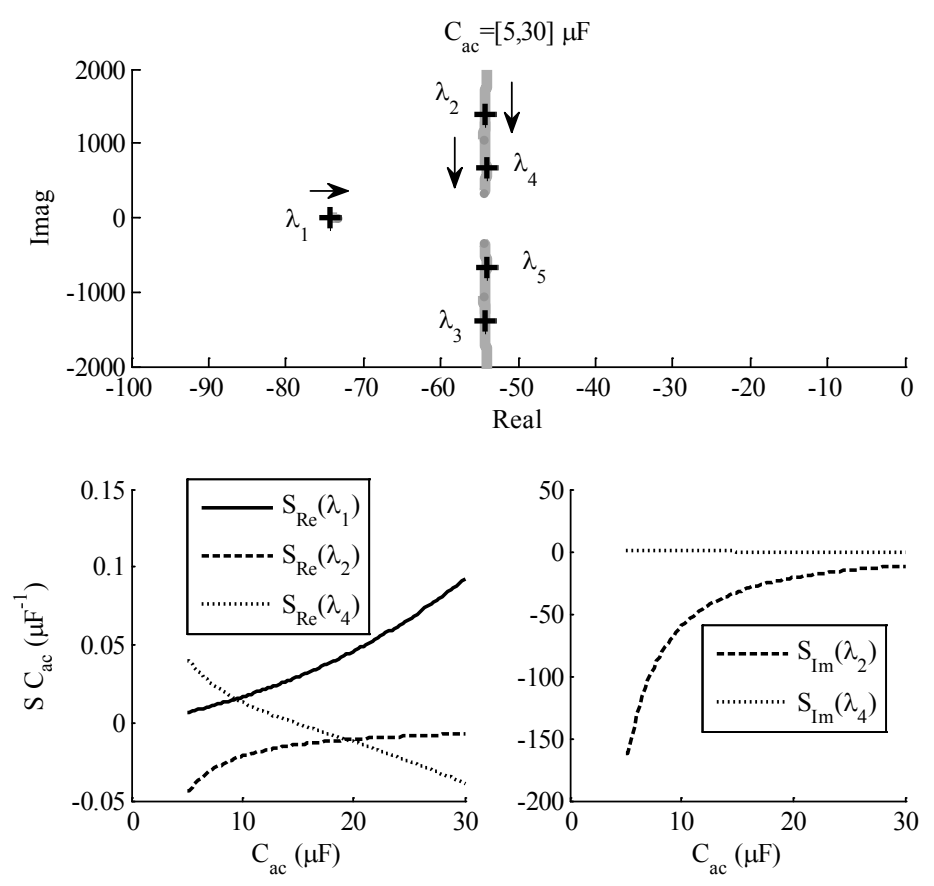

Figure 6.4: Locations of the grid-connected SSBI eigenvalues and their sensitivities versus $C_{a c}$ when it varies between 5 and $30 \mu F$

D. Sensitivity to the Grid Interconnecting Inductor, $R_{g}$ and $L_{g}$

As can be seen in Figure 6.5, increasing the resistance of the grid interconnecting inductor, moves all eigenvalues of the grid-connected three-phase SSBI toward the left. As a result, increasing the resistance improves the stability of the grid-connected SSBI. Again, it should be noted that, increasing the resistance reduces the efficiency of the system, which is usually undesirable. 

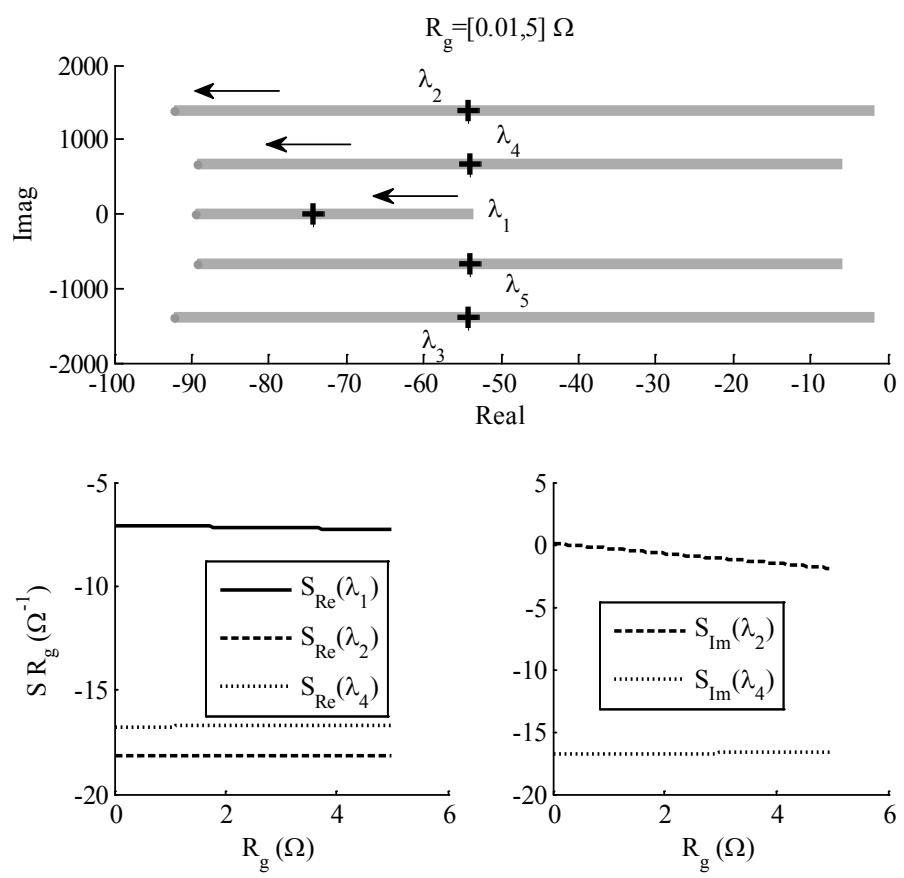

Figure 6.5: Locations of the grid-connected SSBI eigenvalues and their sensitivities versus $R_{g}$ when it varies between 0.01 and $5 \Omega$

The impact of the grid inductance, $L_{g}$, on the location of the systems's eigenvalues is demonstrated in Figure 6.6. It is interesting that unlike the resistive component, increasing the inductive component of interconnecting inductor moves all eigenvalues of the system toward the right-half of the $s$-plane. Thus, increasing the grid resistance aggravates the stability of the grid-connected SSBI. However, it should be noted that, the grid interconnecting inductor has a filter properties, which reduces the injected current harmonics to the grid. Thus, it may not be a good idea to remove the inductor, or reducing its value without considering the quality of the current waveform. Moreover, the grid interconnecting inductor might be used for regulating the power flow from the SSBI to the grid. 

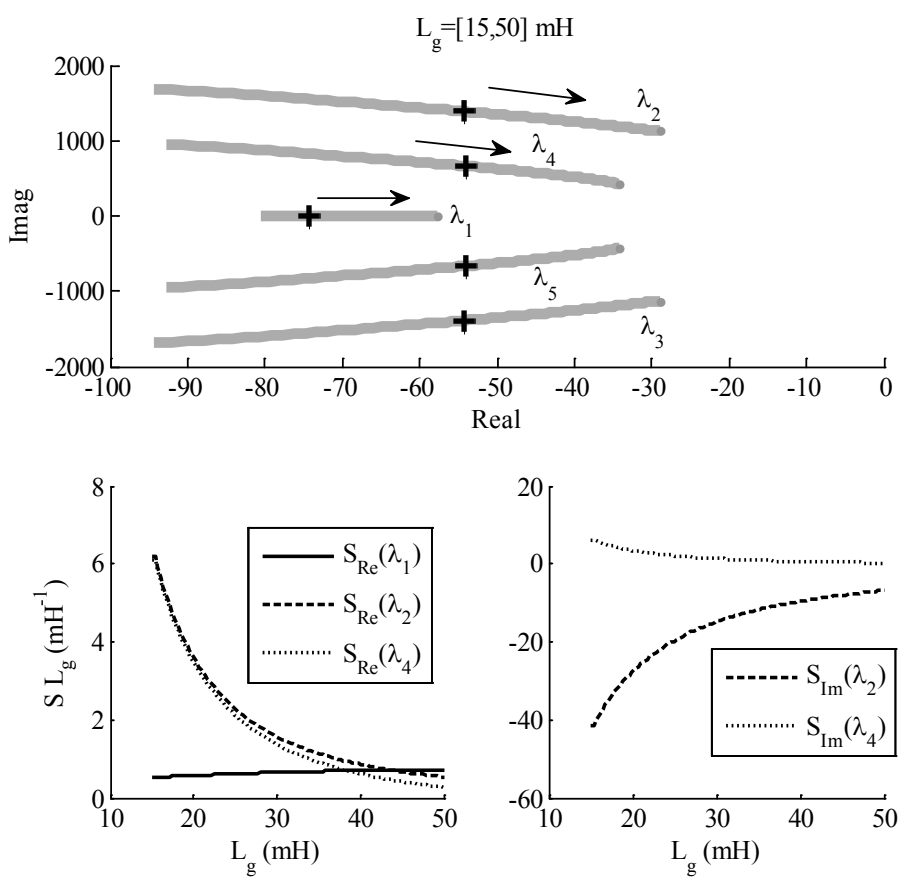

Figure 6.6: Locations of the stand-alone SSBI eigenvalues and their sensitivities versus $L_{g}$ when it varies between 15 and $50 \mathrm{mH}$

\section{E. Sensitivity to the Modulation Index, $M$}

The location of system's eigenvalues as the modulation index varies between 0.15 and 0.30 is shown in Figure 6.7. As can be seen, the complex eigenvalues $\lambda_{2}, \lambda_{3}, \lambda_{4}$, and $\lambda_{5}$ move slightly toward the right, whereas the real eigenvalue $\lambda_{1}$ goes to the opposite way. However, since the four complex eigenvalues have higher weights than the single one, it can be expected that the overall system response becomes slower and more oscillatory as $M$ increases. 

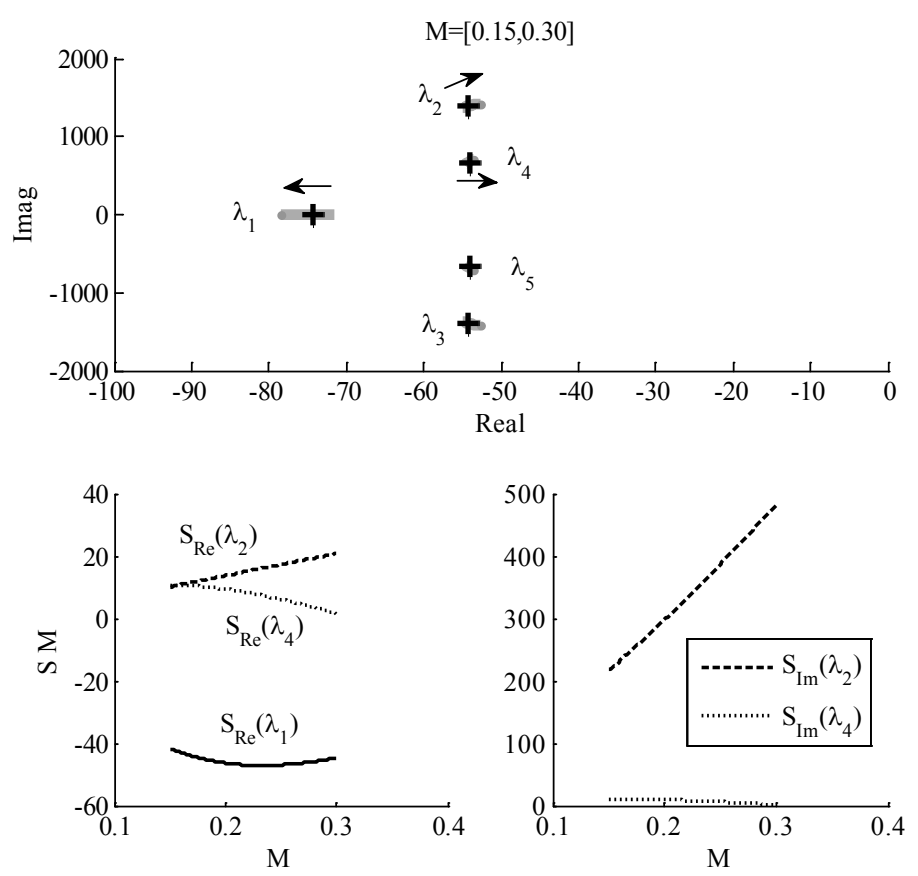

Figure 6.7: Locations of the grid-connected SSBI eigenvalues and their sensitivities versus $M$ when it varies between 0.2 and 0.5

F. Sensitivity to the Modulation Angle, $\Phi$

As can be seen in (6.22), the system matrix, $\overline{\boldsymbol{A}}_{\boldsymbol{d q}}$, is a function of $\Phi$, therefore, one could expect that the eigenvalues of the system displace as the modulation angle varies. However, it is surprising that the system eigenvalues are independent of $\Phi$. In other words, the loci of the system eigenvalues do not change with variations in the modulation index, therefore it does not affect the stability of the grid-connected three-phase SSBI.

\subsection{Simulation and Experimental Results}

In order to verify the validity of the developed state-space-averaged model, a laboratory-scaled grid-connected three-phase SSBI, has been modeled and simulated in 
MATLAB/Simulink. The parameters of the developed set-up are summarized in Table 6.5. The inverter is switched at a frequency of $f_{s}=2880 \mathrm{~Hz}$. These switching signals are calculated and produced using a dSPACE 1104, which operates at a sampling frequency of $f_{\text {sam }}=81 \mathrm{kHz}$.

\section{A. Simulation Results}

The dynamic behavior of the grid-connected SSBI during start-up and its response to step changes in the modulation index, $m$, and the modulation angle, $\phi$, are shown in Figure 6.8. First, the system starts with the nominal parameters of Table 6.5. Second, at $t=100 \mathrm{msec}$, a $3 \%$ step is applied to the modulation index, i.e. $m$ increases from 0.21 to 0.24. Third, at $t=200 \mathrm{msec}$, a $-3^{\circ}$ step change is applied to the modulation angle, i.e. $\phi$ decreases from $60^{\circ}$ to $57^{\circ}$.

It can be seen that the state-space-averaged model can adequately represent the dynamic and steady state behavior of the grid-connected three-phase SSBI. It should be noted that the small-signal model can satisfactorily represent the dynamics of the system during its start-up operation, which is a large-signal disturbance in the dc-source voltage. The reason is that the system and input matrices of the system (6.20), are independent of $v_{d c}$. In other words, the system is linear with respect to $v_{d c}$. Therefore, as long as the other parameters of the system, including $m$ and $\phi$, do not change, the large- and smallsignal transfer functions of the system are the same. Accordingly, the small-signal model of (6.22) can also be used for dynamic analysis of the system under large-signal disturbances in the dc-source voltage. 

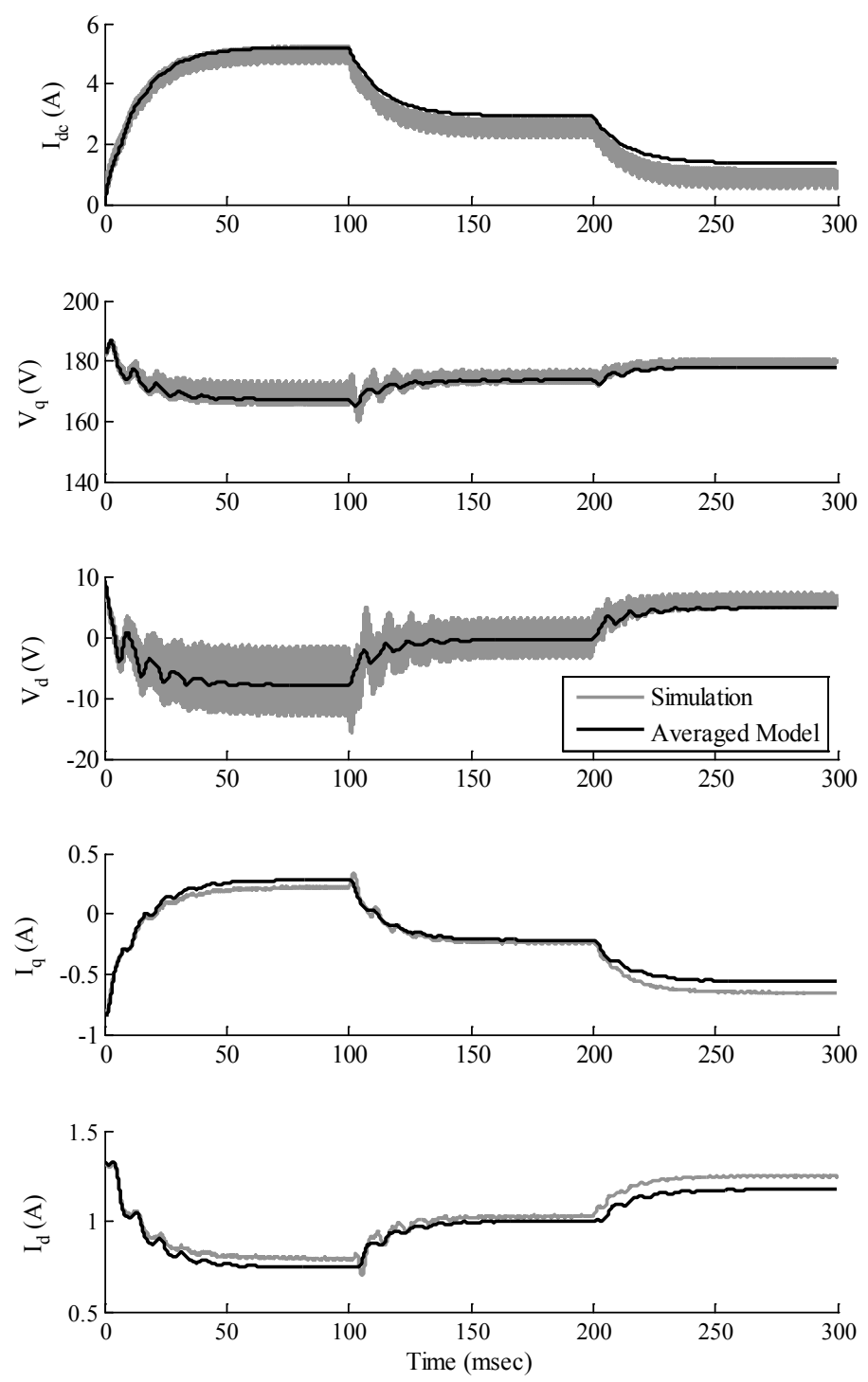

Figure 6.8: State variables of the simulation and state-space-averaged model for $f_{s}=2.88 \mathrm{kHz}$ and

$$
f_{\text {sam }}=2000 \mathrm{kHz}
$$

In order to investigate the impact of the sampling frequencies on the agreement between the state-space averaged model and the circuit simulation, the same simulation has been repeated for two other cases, Case $(i): f_{\text {sam }}=500 \mathrm{kHz}$, and Case $(i i): f_{\text {sam }}=$ $1000 \mathrm{kHz}$. The mean absolute errors (see (4.20)) of the state variables of the grid- 
connected three-phase SSBI during the $300 \mathrm{msec}$ of the simulation are presented in Table 6.6. The results indicate that increasing the ratio of sampling frequency to switching frequency significantly increases the agreement between the state-space-averaged model and simulation data.

Table 6.6: Mean Absolute error of the state-space-averaged model for different sampling frequencies

\begin{tabular}{cccc}
\hline State Variable & $f_{\text {sam }}=500 \mathrm{kHz}$ & $f_{\text {sam }}=1000 \mathrm{kHz}$ & $f_{\text {sam }}=2000 \mathrm{kHz}$ \\
\hline$M A E\left(i_{d c}\right)$ & $0.759 \mathrm{~A}$ & $0.486 \mathrm{~A}$ & $0.367 \mathrm{~A}$ \\
\hline$M A E\left(v_{q}\right)$ & $2.61 \mathrm{~V}$ & $1.952 \mathrm{~V}$ & $1.726 \mathrm{~V}$ \\
\hline$M A E\left(v_{d}\right)$ & $2.42 \mathrm{~V}$ & $1.815 \mathrm{~V}$ & $1.821 \mathrm{~V}$ \\
\hline$M A E\left(i_{q}\right)$ & $0.139 \mathrm{~A}$ & $0.0788 \mathrm{~A}$ & $0.0530 \mathrm{~A}$ \\
\hline$M A E\left(i_{d}\right)$ & $0.094 \mathrm{~A}$ & $0.0625 \mathrm{~A}$ & $0.0479 \mathrm{~A}$ \\
\hline
\end{tabular}

\section{B. Experimental Results}

The state-space-averaged models of (6.20) and (6.22) have been derived for an ideal grid-connected three-phase SSBI, in which all voltage drops and delays of the semiconductor devices (switches and diodes) have been neglected. However, these assumptions may not be true in practice. In other words, there are conduction losses and voltage drops across the semiconductor switches and diodes during conductions. The existing non-idealities, like conduction losses and voltage drops of the semiconductors, turn-on and turn-off delays, diode reverse-recovery currents, snubber circuits, and imperfect switching, affect the accuracy of the derived state-space-averaged model. Our studies have shown that the voltage drops and losses of the semiconductor devices could be represented by considering their forward bias resistances in the model and the effects of the snubbers and imperfect switching would be incorporated into the averaged model through a slight change in the modulation index, $m$. The dynamics of the start-up 
operation (large-signal disturbance in the dc-source voltage) of the laboratory-scaled three-phase SSBI, along with the response of the modified state-space-averaged model, have been depicted in Figures 6.9 through 6.13, and the hardware data is presented in Table 6.7.

Table 6.7: Hardware specifications

\begin{tabular}{cc}
\hline Component & Specifications \\
\hline dc-source & XR125-32 Magna-Power Electronics \\
\hline MOSFETS & STx42N65M5 (TO-220) \\
\hline Diodes & DSEP30-06A (TO-247 AD) \\
\hline Simulink-Hardware Interface & dSPACE 1104 \\
\hline$C_{\text {snubber }}$ & $100 n F$ \\
\hline$D_{\text {snubber }}$ & $1 \mathrm{~N} 4004-\mathrm{G}$ \\
\hline$R_{\text {snubber }}$ & $240 \Omega$ \\
\hline
\end{tabular}

The aforementioned modifications can be applied as follows:

i. According to the datasheets [81-82], the forward bias resistances of the MOSFETs and diodes, for a $5 A$ current, are $0.065 \Omega$ and $0.225 \Omega$, respectively. Since, at each instant two diodes and two switches are in series between the dc-source and the ac-side of the circuit, the total resistance of the semiconductor devices will be $0.58 \Omega$. Thus, considering $0.1 \Omega$ resistance for the dc-link inductor, the total/equivalent series resistance of the dc-link will be $0.68 \Omega$.

ii. The modulation index must increase by $1 \%$ in the model to include the delay and imperfect switching effects. In other words, the experimental waveforms of the three-phase SSBI system with a modulation index of 
$m=0.20$ correspond to the waveforms of the state-space-averaged model for and $m=0.21$.
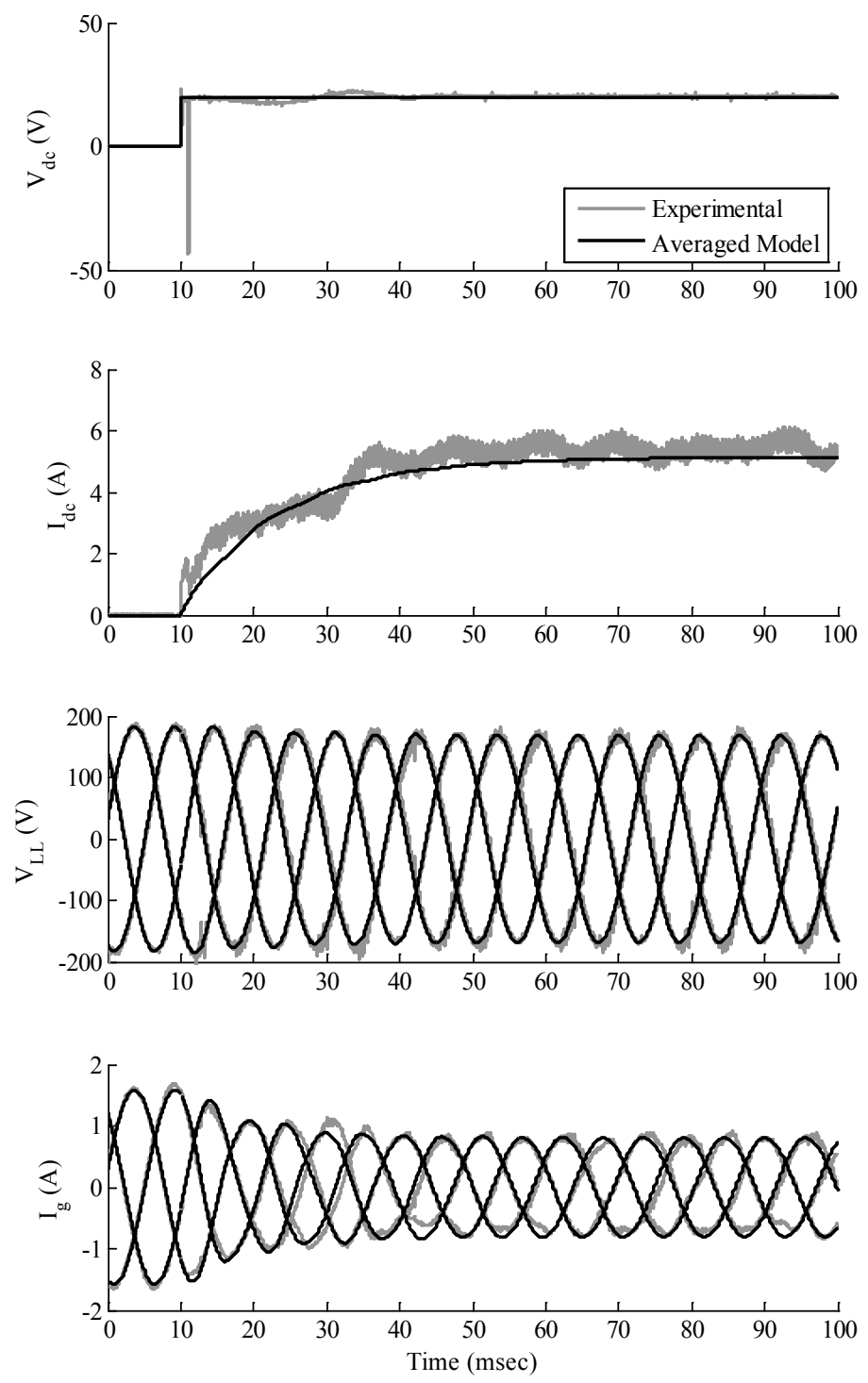

Figure 6.9: Start-up operation of the lab-scaled circuit and the response of the state-space-averaged model (with an ideal dc-source) for $f_{s}=2.88 \mathrm{kHz}$ and $f_{\text {sam }}=81 \mathrm{kHz}$ 

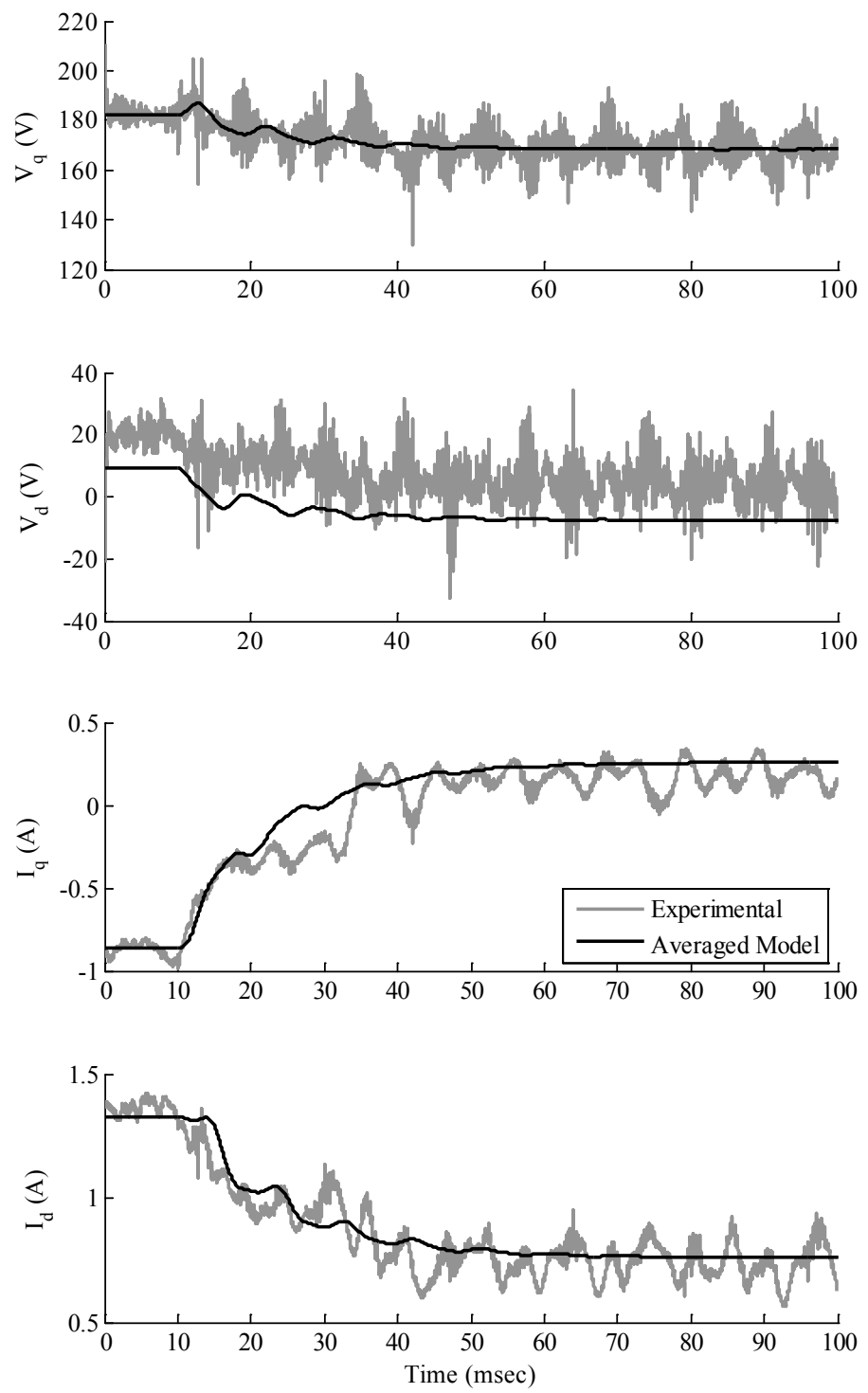

Figure 6.10: $d q$ state variables of the lab-scaled circuit and the state-space-averaged model (with an ideal dc-source) during the start-up for $f_{s}=2.88 \mathrm{kHz}$ and $f_{\text {sam }}=81 \mathrm{kHz}$

The experimentally obtained data from the prototype circuit and the state-spaceaveraged model are plotted in Figure 6.9 and 6.10. As can be seen in these figures, the state-space-averaged model can adequately describe the dynamic behavior of the grid- 
connected SSBI system. The minor differences between the model and real data arise from the inherent limitations of the state-space averaging method (in determining the high frequency characteristics of the system), unconsidered non-idealities of the system, such as the imperfect switching of the dc-source and its transients during the start-up, low sampling frequency, the parasitic and unbalanced elements of the circuit, as well as harmonic content and unbalance in the grid that cause distortions and low frequency ripples in the dc-link current and $d q$ variables. In fact, the harmonic content was the source of major problems in performing the grid-connected experiments. The available threephase grid in the laboratory had some low order voltage harmonics that excited the resonance frequency $(156 \mathrm{~Hz})$ of the $\mathrm{LC}$ circuit, consisting of the grid interconnecting inductor and the ac-side capacitor. This resonance produced large harmonic currents between the LC circuit and the grid, even when the SSBI was off. As can be seen in Figure 6.10, these harmonics caused considerable ripples on the grid current. However, the state-space-averaged model can successfully follow the dc component of $I_{q}$ and $I_{d}$. The differences between the model and the actual circuit may become more noticeable in the small-signal response of the system, in which the variations of the state-variables are comparable with the ripples and distortions. This can be realized in Figures 6.11 and 6.12, where the response of the prototyped grid-connected three-phase SSBI to small-signal disturbances in the modulation index, $m$ ( $3 \%$ increase), and the modulation angle, $\phi\left(3^{\circ}\right.$ decrease), has been illustrated. However, the model response is still reasonably acceptable for many applications. 

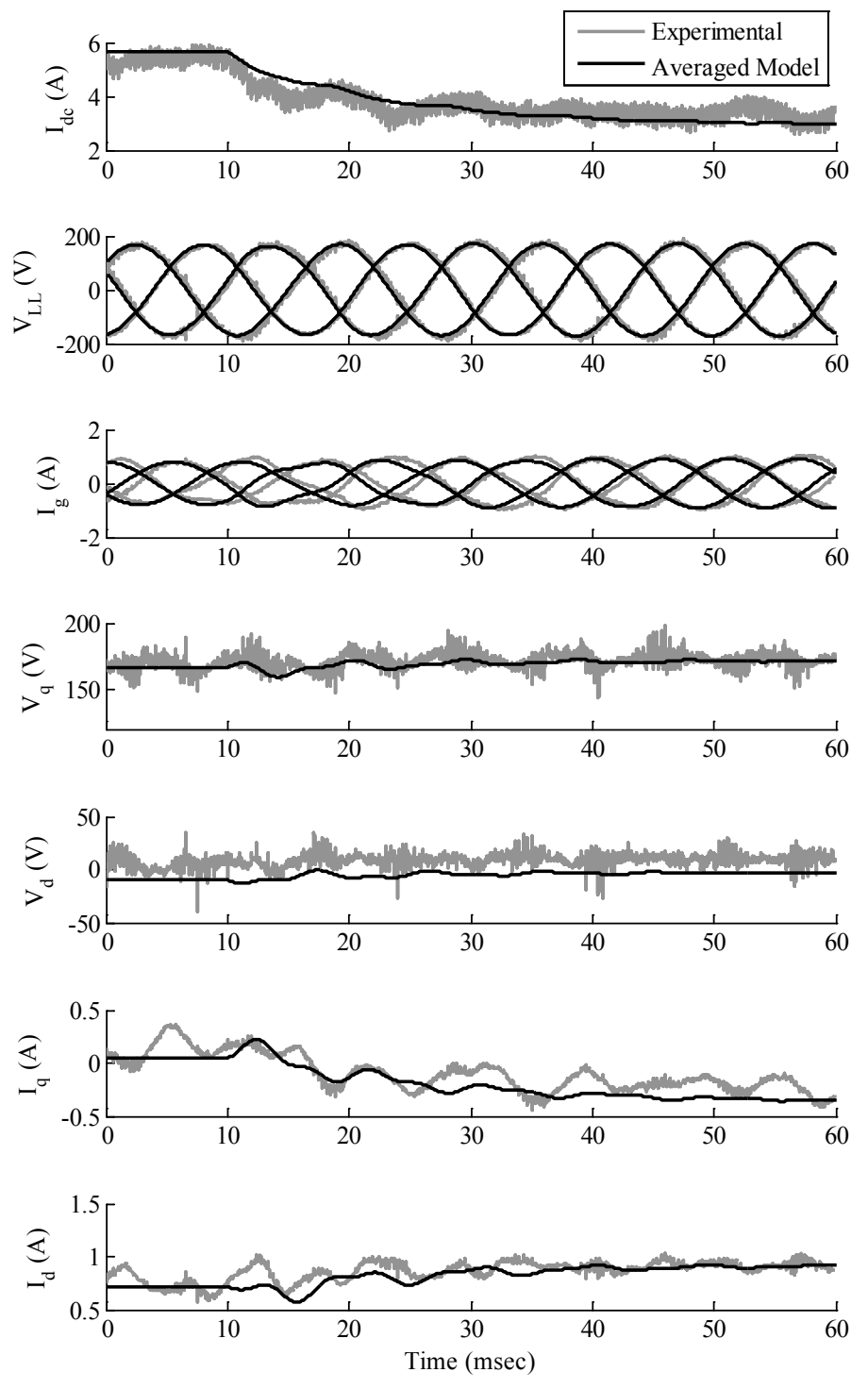

Figure 6.11: Step responses of the hardware and the state-space-averaged model, to a $3 \%$ increase in $m$, for

$$
f_{s}=2.88 \mathrm{kHz} \text { and } f_{\text {sam }}=81 \mathrm{kHz}
$$



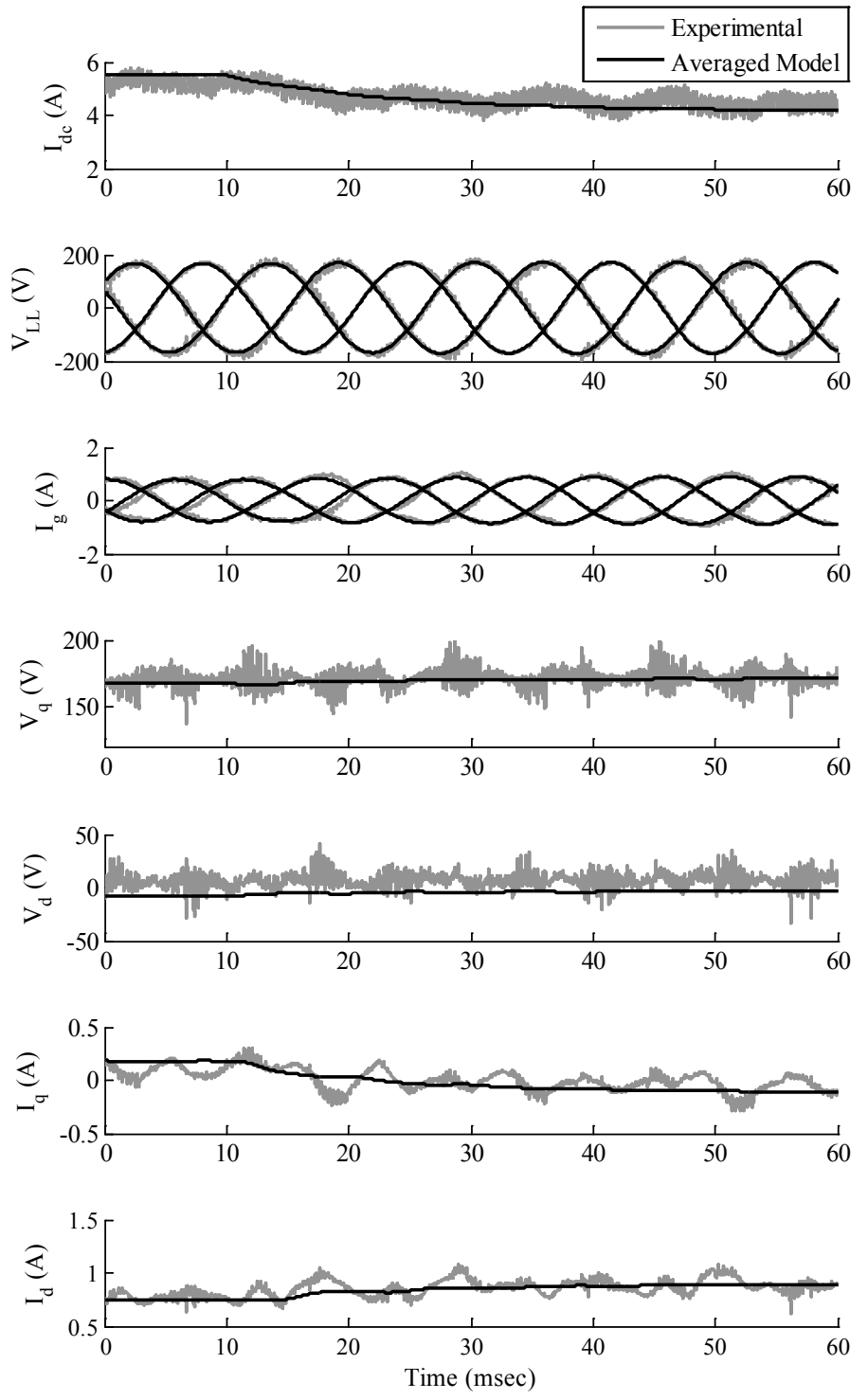

Figure 6.12: Step responses of the hardware and the state-space-averaged model, to a $-3^{\circ}$ decrease in $\phi$, for

$$
f_{s}=2.88 \mathrm{kHz} \text { and } f_{\text {sam }}=81 \mathrm{kHz}
$$



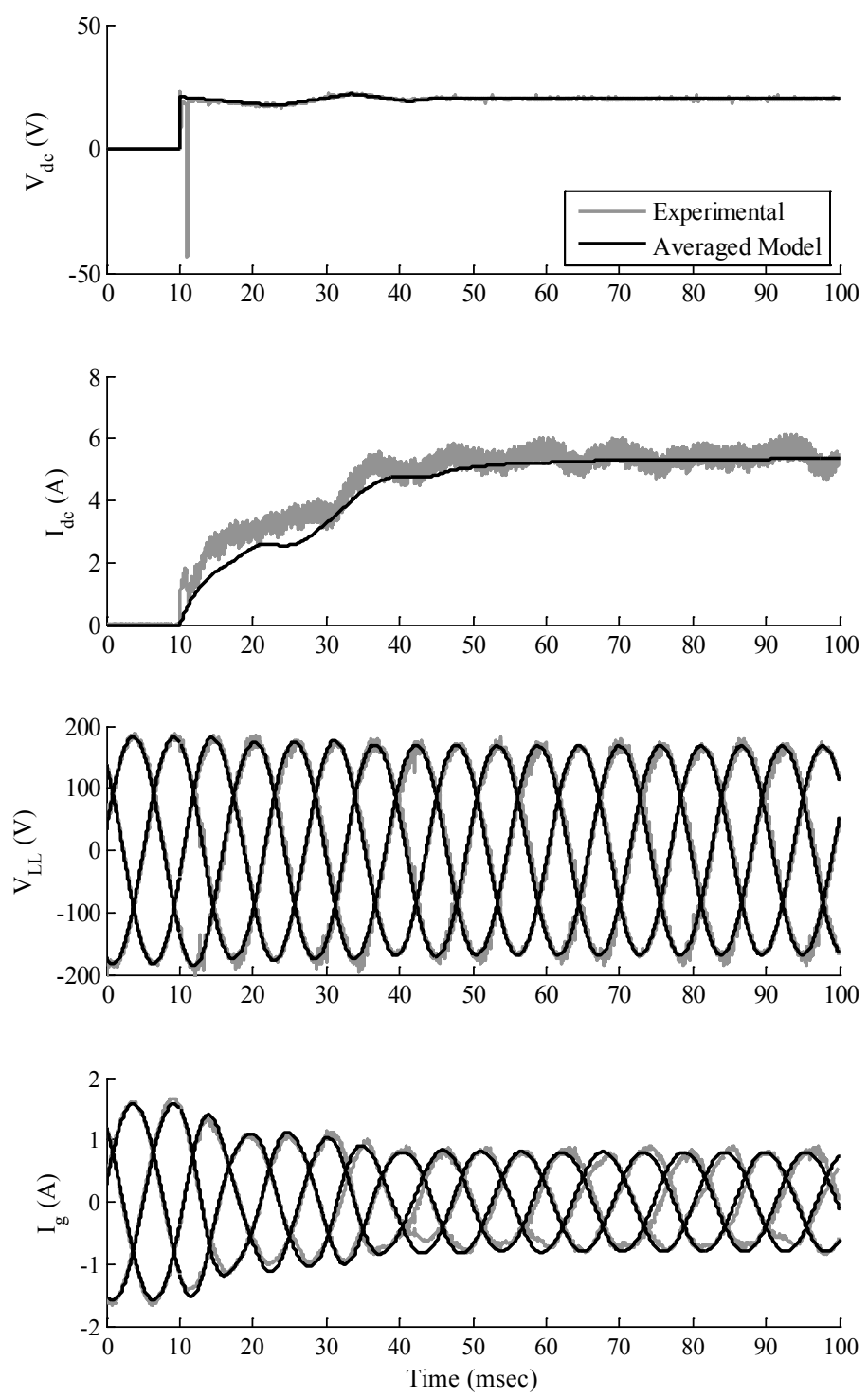

Figure 6.13: Start-up operation of the lab-scaled circuit and the response of the state-space-averaged model, considering the dc-source abnormality, for $f_{s}=2.88 \mathrm{kHz}$ and $f_{\text {sam }}=81 \mathrm{kHz}$

As was already mentioned, a variety of non-idealities can affect the operation of the SSBI and accuracy of the developed state-space averaged models. Abnormal operation 
of the dc-source, besides the semiconductor and snubber effects, is another non-ideality that can be incorporated into the developed state-space-averaged model.
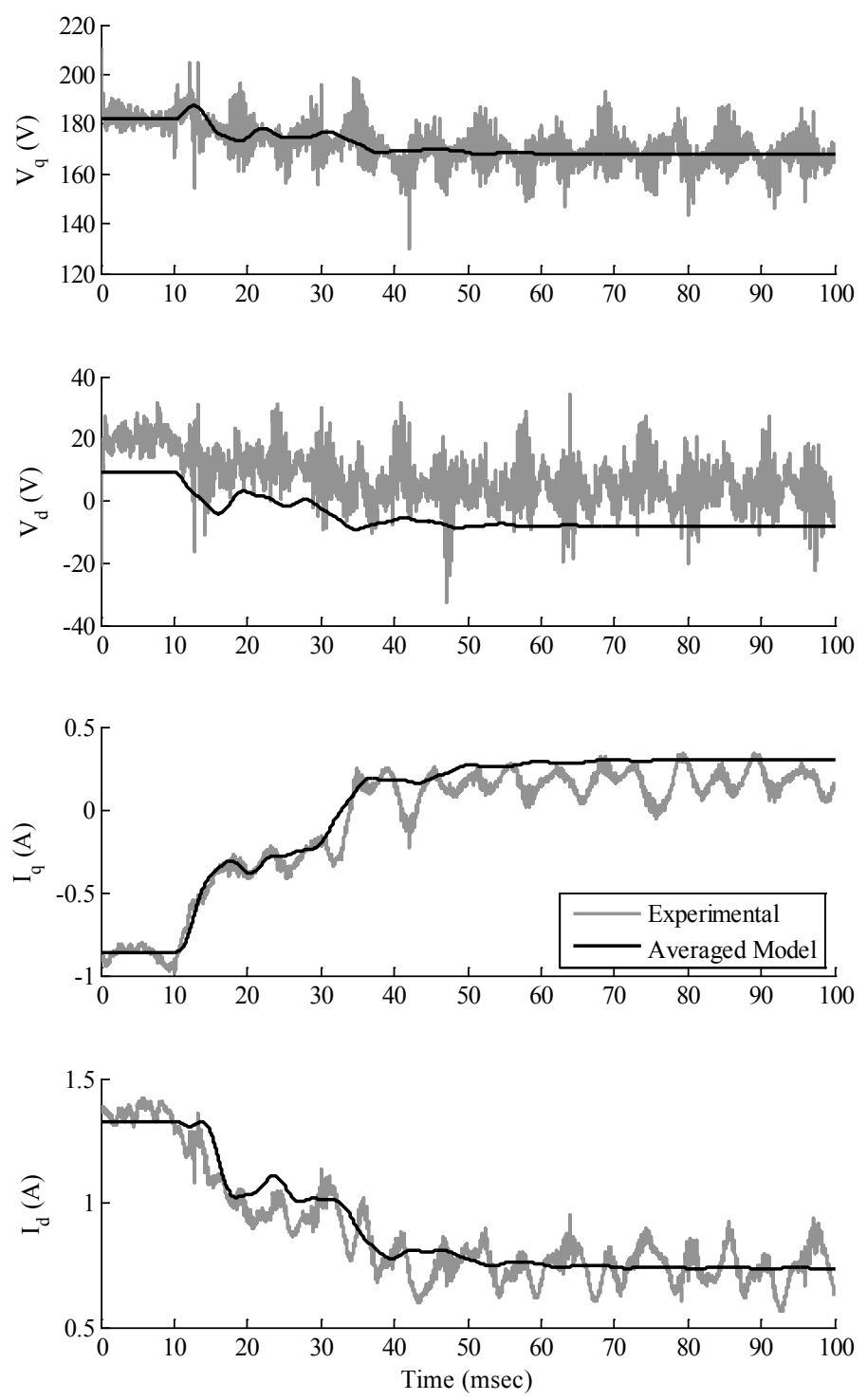

Figure 6.14: $d q$ state variables of the lab-scaled circuit and the state-space-averaged model, considering the dc-source abnormality, during the start-up for $f_{s}=2.88 \mathrm{kHz}$ and $f_{\text {sam }}=81 \mathrm{kHz}$ 
Looking at Figure 6.9 indicates that the dc-source voltage does not behave perfectly once it is switched to the circuit, and it takes about $50 \mathrm{msec}$ until the start-up transients disappear and the voltage settles on $20 \mathrm{~V}$. The state-space-averaged models of (6.20) and (6.22) are capable of considering this abnormal operation, by means of inputting the emulated waveform of the actual dc-source into the model. This has been shown in Figures 6.13 and 5.14. As can be seen, and in comparison with Figures 6.9 and 6.10, considering the dc-source transients results in a noticeable improvement in the model performance.

\subsection{Summary}

The state-space averaging method has been applied in order to develop the dynamic model of the grid-connected three-phase SSBI. The large- and small-signal models have been derived in the synchronous $d q$-frame of reference, and used for sensitivity and stability analysis of the system. It has been observed that, unlike the standalone system, the grid-connected single-stage boost inverter does not have non-dominant eigenvalues. A modification approach for taking into account the losses and nonidealities of the semiconductor devices, has been proposed and has been validated through simulated and experimentally obtained data. Finally, it has been shown that the

obtained models can take into account the abnormal operation of the dc-source. Following chapter will be a conclusion to this research and some issues and ideas are addressed as potential subjects for future work. 


\section{CHAPTER 7}

\section{CONCLUSION AND FUTURE WORK}

The conclusion of this $\mathrm{PhD}$ dissertation is presented in this chapter. The achievements and contributions of the work are revisited and some suggestions are made for future work.

\subsection{Conclusion}

The single-stage boost inverter (SSBI) is an appropriate solution for sustainable energy-based generation systems in which a low dc voltage has to be converted to higher ac voltage. This technology is particularly suitable for residential photovoltaic- and fuel cell-based SE systems. In order to investigate the stability and dynamic performance of the SSBI, and design a proper control system, it is essential to develop a dynamic model for it. This dissertation presented the results of a comprehensive study on dynamic modeling and analysis of the SSBI systems. The following are the major contribution of this study:

i. Development of large- and small-signal state-space-averaged models for the stand-alone and grid-connected single-stage boost inverters.

ii. Analysis of the stability and dynamic behavior of the stand-alone and gridconnected SSBI by performing eigenvalue sensitivity analysis.

These were achieved through exploring different aspects of the problem and adopting suitable means of solving it. At the first step, a comprehensive review of the existing PWM-based power conversion systems was performed. The focus of the survey 
was on the converter topologies, which are used in sustainable energy systems with a low dc voltage, e.g. solar photovoltaic and fuel cell systems.

Besides the existing topologies, extensive research was carried out on the history and contributions of the available methodologies for steady-state and dynamic modeling of the power electronic converters. It was concluded that the state-space averaging, and its variations, are by far the most used techniques for dynamic modeling of the switchmode power converters. This is due to (1) the strength of the state-space averaging method in solving a broad spectrum of modeling problems, and (2) its convenience of implementation.

The idea of the SSBI and the recently proposed PPWM-based switching pattern, which has three charging and six discharging states, were elaborated upon, and the fundamental equations of the system were derived and verified through simulations and experiments. It was shown that this topology, along with the given switching pattern, is capable of providing the residential ac voltage from a single photovoltaic panel at its rated power.

The idea of the SSBI was extended to the single-phase SSBI and the switching pattern is adapted for the single-phase application. In this case, there is one charging and one discharging states during each switching cycle, where only one switch operates at high frequency, one does not conduct, and two switches conduct for the entire switching cycle. This system was simulated, tested, and verified for grid-connected application.

The possible switching states of the stand-alone three-phase SSBI were enumerated, and the associated state-space equations were derived and averaged over a switching cycle. The obtained time-varying averaged model was transformed into the 
synchronous $d q$-frame of reference in order to derive the time-invariant large-signal statespace-averaged model of the system. The small-signal state-space-averaged model of the SSBI is developed by means of perturbation and linearization of the large-signal model. This model was used for eigenvalue sensitivity analysis in order to investigate the stability and dynamic behavior of the stand-alone three-phase SSBI system. It was observed that, the stand-alone system has three dominant and two non-dominant eigenvalues, in which the location of the non-dominant eigenvalues are only affected by the load parameters. A modification approach for taking into account the losses and nonidealities of the semiconductor devices, was proposed and validated through simulation and experiments.

The methodology was applied to the grid-connected three-phase single-stage boost inverter, which is more complicated than its stand-alone counterpart, and the results were successfully tested on the laboratory-scaled hardware setup. Unlike the stand-alone system, the grid-connected SSBI does not have non-dominant eigenvalues. Finally, the abnormal operation of the dc-source during the start-up was studied and it was observed that the developed state-space-averaged models are capable of taking into account this type of abnormality.

\subsection{Future Work}

Many problems were identified over more than one year of working on this research work. Of course, some of them were solved during the course of the study, however the rest of the problems can be the subjects of future studies. 
- Voltage spikes, across the semiconductor switches and diodes, are one of the major problems in design and implementation of the SSBI. Turn-off RDC snubber circuits have been employed in order to reduce the problem. Although, they significantly reduced the voltages spikes and failures of the circuit, there is still room for further studies and the design of more elaborate snubbers for the SSBI.

- A variety of optimization objectives can be defined and the circuit can be accordingly optimized. Tentative objectives can be maximizing efficiency or minimizing cost and size. This subject can be combined with the snubber design, because snubbers affect the efficiency, size, and cost of the circuit. Alternatively, finding a proper switch-diode combination can reduce the need for snubber and can improve the efficiency, size, cost, and reliability of the circuit. Thus, the switching/speed characteristics of the semiconductor devices should be considered in the optimization problems.

- Improving the switching pattern is another viable option for enhancing the system performance. It may make the SSBI more efficient or increase the quality of the output waveforms, which will result in a simpler filter design. In addition, an improved switching pattern can reduce the voltage spikes and the snubber size.

- The developed state-space-averaged models can be further elaborated by considering more details, like parasitic elements of the circuit, or discontinuous mode of operation (however, the discontinuous operation has been never observed during this research). 
- Different control studies (such as closed loop control, optimal and adaptive controls, and observability and controllability) can be performed by use of the obtained state-space-averaged models. For instance, a nonlinear control algorithm like sliding-mode control can be applied in order to control the active and reactive powers in the grid-connected mode, or to control the voltage in the stand-alone operation.

- Different faults of the system should be studied, and a proper protection system should be designed for the SSBI. For example, an open circuit fault can cause overvoltage across the ac-side capacitors. Thus, this fault must be detected by the protection system, and a proper action (like by turning the switches off) must be taken.

- Fault-ride-through and transient stability analyses as well as the seamless transition between the grid-connected and stand-alone operations could be some other interesting subjects for future work. 


\section{BIBLIOGRAPHY}

[1] Q. Li, and P. Wolfs, "A review of the single phase photovoltaic module integrated converter topologies with three different DC link configurations", IEEE Transactions on Power Electronics, vol. 23, no. 3, pp. 1320-1333, May. 2008.

[2] S. Alepuz, S.B. Monge, J. Bordonau, J. Gago, D. Gonzalez, and J. Balcells, "Interfacing renewable energy sources to the utility grid using a three-level inverter," IEEE Transactions on Indsutrial Electronics, vol. 53, no. 5, pp. 15041511, Oct. 2006.

[3] F.Z. Peng, "Z-source inverter," IEEE Transactions on Industry Applications, vol. 39, no. 2, pp. 504-510, Mar./Apr. 2003.

[4] B. Mirafzal, M. Saghaleini, A.K. Kaviani, "An SVPWM-based switching pattern for stand-alone and grid-connected three-phase single-stage boost-inverter," IEEE Transactions on Power Electronics, vol. 26, no. 4, pp.1102-1111, Apr. 2011.

[5] A.K. Kaviani, B. Mirafzal, "A Switching Pattern for Single-Phase Single-Stage Current-Source Boost-Inverter," 27th Annual IEEE Applied Power Electronics Conference \& Exposition, pp. 2066-2071, 2012.

[6] G. Petrone, G. Spagnuolo, R. Teodorescu, M. Veerachary, and M. Vitelli, "Reliability issues in photovoltaic power processing systems," IEEE Transactions on Industrial Electronics, vol. 55, no. 7, pp. 2569-2580, July 2008.

[7] M. Calais, J. Myrzik, T. Spooner, and V. G. Agelidis, "Inverters for single-phase grid connected photovoltaic systems-an overview," in Proc. IEEE 33rd Annual Power Electronics Specialists Conference, Jun. 2002, vol. 4, no. 23-27, pp. 19952000.

[8] M. Calais, V.G. Agelidis, "Multilevel converters for single-phase grid connected photovoltaic systems-an overview," IEEE International Symposium on Industrial Electronics (ISIE '98), vol. 1, pp. 224-229, Jul. 1998.

[9] S. Alepuz, J. Bordonau, and J. Peracaula, "Dynamic analysis of three-level voltage-source inverters applied to power regulation," Proceedings of IEEE Power Electronics Specialist Conference, pp. 721-726, 1999.

[10] S. Alepuz, J. Bordonau, and J. Peracaula, "A novel control approach of three-level VSIs using a LQR-based gain-scheduling technique," Proceedings of IEEE Power Electronics specialist Conference, pp. 743-748, 2000. 
[11] J. Rodríguez, J.S. Lai, and F.Z. Peng, "Multilevel inverters: A survey of topologies, controls, and applications," IEEE Transactions on Industrial Electronics, vol. 49, no. 4, pp. 724-738, Aug. 2002.

[12] M. Mohr, W.T. Franke, B. Witting, and F.W. Fuchs, "Converter systems for fuel cells in the medium power range - a comparative study," IEEE Transactions on Industrial Electronics, vol. 57, no. 6, pp. 2024-2032, Jun. 2010.

[13] Y. Huang, M. Shen, F.Z. Peng, and J. Wang, "Z-source inverter for residential photovoltaic systems," IEEE Transactions on Power Electronics, vol. 21, no. 6, pp. 1776-1782, Nov. 2006.

[14] R. Badin, Y. Huang, F.Z. Peng, and H. Kim, "Grid interconnected Z-source PV system," Proceedings of IEEE 38th Annual Power Electronics Specialist Conference, vol. 1, pp. 2328-2333, Jun. 2007.

[15] C.J. Gajanayake, D.M. Vilathgamuwa, P.C. Loh, R. Teodorescu, and F. Blaabjerg, "Z-source-inverter-based flexible distributed generation system solution for grid power quality improvement," IEEE Transactions on Energy Conversion, vol. 24, no. 3, pp. 695-704, Sep. 2009.

[16] B. Sahan, A. Vergara, N. Henze, A. Engler, and P. Zacharias, "A single-stage PV module integrated converter based on a low-power current-source inverter," IEEE Transaction on Industrial Electronics, vol. 55, no. 7, pp. 2602-2609, Jul. 2008.

[17] Y. Chen, and K. Smedley, "Three-phase boost-type grid-connected inverter," IEEE Transactions on Power Electronics, vol. 23, no. 5, pp. 2301-2309, Sep. 2008.

[18] M. Kazerani, Z.C. Zhang, and B.T. Ooi, "Linearly controllable boost voltages from tri-level PWM current-source inverter," IEEE Transactions on Industrial Electronics, vol. 42, no. 1, pp. 72-77, Feb. 1995.

[19] W. Libo, Z. Zhengming, and L. Jianzheng, "A single-stage three-phase gridconnected photovoltaic system with modified MPPT method and reactive power compensation," IEEE Transactions on Energy Conversion, vol. 22, no. 4, pp. 881-886, Dec. 2007.

[20] A.K. Kaviani, K. Yen, B. Mirafzal, "Dynamic model of three-phase current source boost inverter for stand-alone applications," 27th Annual IEEE Applied Power Electronics Conference \& Exposition, pp. 218-224, 2012.

[21] Z. Qin, D. Sha, X. Liao, "A three-phase boost-type grid-connected inverter based on synchronous reference frame control," 27th Annual IEEE Applied Power Electronics Conference \& Exposition, pp. 384-388, 2012. 
[22] M. Saghaleini, B. Mirafzal, "Reactive power control in three-phase gridconnected current source boost inverter," 27th Annual IEEE Applied Power Electronics Conference \& Exposition, pp. 904-910, 2012.

[23] P.T. Krein, J. Bentsman, R.M. Bass, B.L. Lesieutre, "One the use of averaging for the analysis of power electronic systems," IEEE Transaction os Power Electronics, vol. 5, no. 2, pp.182-190, Apr. 1990.

[24] R.W. Erickson, "DC-DC power converters," Article in Wiley Encyclopedia of Electrical and Electronics Engineering.

[25] N. Mohan, T.M. Undeland, W.P. Robbins, Power Electronics: Converters, Applications, and Design, John Wiley and sons INC, Third edition, Hoboken NJ, USA, 2003

[26] J. Holts, "Pulsewidth modulation-a survey," IEEE Transactions on Industrial Electronics, vol. 39, no. 5, pp. 410-420, Dec. 1992.

[27] D. G. Holmes, and T. A. Lipo, Pulse width modulation for power converters: principles and practice, IEEE Press, Wiley-IEEE, 2003.

[28] P. C. Krause, O. Wasynczuk, S.D. Sudhoff, Analysis of Electric Machinery and Drive Systems, IEEE Press, Wiley-Interscience, Second edition, 2002.

[29] J.A. Sanders, F. Verhulst, Averaging Methods in Nonlinear Dynamical Systems, New York, Springer-Verlag, 1985.

[30] P.T. Krein, J. Bentsman, R.M. Bass, B.L. Lesieutre, "One the use of averaging for the analysis of power electronic systems," IEEE Transactions on Power Electronics, vol. 5, no. 2, pp.182-190, Apr. 1990.

[31] B. Lehman, R.M. Bass, "Extension of averaging theory for power electronic systems," IEEE Transactions on Power electronics, vo. 11, no. 4, pp. 542-553, July 1996.

[32] R.D. Middlebrook, S. Cuk, "A general unified approach to modeling switchingconverter power stages," Proceedings of IEEE Power Electronics Specialists Conference, pp. 18-34, 1976.

[33] B. Lehman, R.M. Bass, "Recent advances in averaging theory for PWM dc-dc converters," Proceeding of the $35^{\text {th }}$ IEEE conference on decision and control, vol. 4, pp. 4467-4471, Dec. 1996. 
[34] B. Lehman, R.M. Bass, "switching frequency dependent averaged models for PWM dc-dc converters," IEEE Transactions on Power electronics, vol. 11, no. 1, pp. 89-98, Jan. 1996.

[35] V.A. Calisikan, G.C. Verghese, A.M. Stankovic, "Multifrequency averaging of dc/dc converter," IEEE Transactions on Power electronics, vol. 14, no. 1, pp. 124-133, Jan. 1999.

[36] A. Davoudi, J. Jatskevich, "Parasitic realiziation in state-space average-value modeling of PWM dc-dc converters using an equal area method," IEEE Transactions on Circuit and Systems-1, vol. 54, no. 9, pp. 1960-1967, Sep. 2007.

[37] H. Sira-Ramirez, M. Delgado de Nieto, "A Lagrangian approach to average modeling of pulsewidth-modulation controlled dc-to-dc power converters," IEEE Transactions on Circuits and Systems-1, vol. 43, no. 5, pp. 427-430, May 1996.

[38] R.D. Middlebrook, "Small-signal modeling of pulse-width modulated switchedmode power converters," Proceedings of IEEE, vol. 76, no. 4, pp. 343-354, Apr. 1988.

[39] V. Vorperian, R. Tymerski, F.C.Y. Lee, "Equivalent circuit models for resonant and PWM switches," IEEE Transactions on Power Electronics, vol. 4, no. 2, pp. 205-214, Apr. 1989.

[40] R. Tymersky, V. Vorperian, F.C.Y. Lee, W.T. Baumann, "Nonlinear modeling of the PWM switch," IEEE Transactions on Power Electronics, vol. 4, no. 2, pp. 225-233, Apr. 1989.

[41] S.R. Sanders, J.M. Noworolski, X.Z. Liu, G.C. Verghese, "Generalized averaging method for power conversion circuits," IEEE Transactions on Power Electronics, vol. 6, no. 2, pp. 251-259, Apr. 1991.

[42] J. Mahdavi, S. Emaadi, M. Ehsani, "Analysis of power electronic converters using the generalized state-space averaging approach," IEEE Transactions on Circuits and Systems-1, vol. 44, no. 8, pp. 767-770, Aug. 1997.

[43] Z. Mihajlovic, B. Lehman, C.S. Sun, "Output ripple analysis of switching dc-dc converters," IEEE Transaction on Circuits and Systems-1, vol. 51, no. 8, pp. 1596-1611, Aug. 2004.

[44] D. Maksimovich, S. Cuk, "A unified analysis of PWM converters in discontinuous modes," IEEE Transactions on Power Electronics, vol. 6, no. 2, pp. 479-490, Jul. 1991. 
[45] N. Femia, V. Tucci, "On the modeling of PWM converters for large signal analysis in discontinuous mode," IEEE Transactions on Power Electronics, vol. 9, no. 5, pp. 487-496, Sep. 1994.

[46] A. davoudi, J. Jatskevich, T. De Rybel, "Numerical state-space average-value modeling of PWM dc-dc converters operating in DCM and CCM," IEEE Transactions on Power electronics, vol. 21, no. 4, pp. 1003-1012, July 2006.

[47] N. Femia, G. Spagnuolo, v. Tucci, "State-space models and order reduction for dc-dc switching converters in discontinuous modes," IEEE Transactions on Power Eelctronics, vol. 10, no. 6, pp. 640-650, Nov. 1995.

[48] J. Sun, D.M. Mitchell, M.F. Greuel, P.T. Krein, R.M. Bass, "Averaged modeling of PWM converters operating in discontinuous conduction mode," IEEE Transactions on Power eelctronics, vol. 16, no. 4, pp. 482-492, July 2001.

[49] K.D.T, Ngo, "Low frequency characterization of PWM converters," IEEE Transactions on Power Electronics, vol. PE-1, no. 4, pp. 223-230, Oct. 1986.

[50] S. Hiti, D. Boroyevich, C. Cuadros, "Small-signal modeling and control of threephase PWM converters," IEEE Industry Applications Society Annual Meeting, pp. 1143-1150, vol. 2, 1994.

[51] S. Hiti, D. Boroyevich, "Control of front-end three-phase boost rectifier," 9th Annual IEEE Applied Power Electronics Conference \& Exposition, pp. 927-933, 1994.

[52] S. Chiniforoosh, H. Atighechi, A. Davoudi, J. Jatskevich, A. Yazdani, S. Filizadeh, M. Saeedifard, J.A. Martinez, V. Sood, K. Strunz, J. Mahseredjian, D. Dinavahi, "Dynamic average modeling of front-end diode rectifier loads considering discontinuous conduction mode and unbalanced operation," IEEE Transactions on Power Delivery, vol. 27, no. 1, pp. 421-429, Jan. 2012.

[53] C.T. Rim, D.Y. Hu, G.Y. Cho, "Transformer as equivalent circuits for switches: general proofs and d-q transformation-based analyses," IEEE Transactions on Industry Applications, vol. 26, no. 4, pp. 777-785, July/Aug. 1990.

[54] H. Mao, D. Boroyevich, F.C.Y Lee, "Novel reduced-order small-signal model of a three-phase PWM rectifier and its application in control design and system analysis," IEEE Transactions on Power Electronics, vol. 13, no. 3, pp. 511-521, May 1998.

[55] G. Venkataramanan, B. Wang, "Dynamic modeling and control of three phase pulse width modulated power converters using phasors," 35th Annual IEEE Power Electronics Specialists Conference, vol.4, no., pp. 2822- 2828, 2004. 
[56] A.M. Stankovich, S.R. Sanders, T. Aydin, "Dynamic phasors in modeling and analysis of unbalanced polyphase ac machines," IEEE Transactions on Energy Conversion, vol. 17, no. 1, pp. 107-113, Mar. 2002.

[57] J. Bordonau, M. Cosan, D. Borojevic, H. Mao, F.C. Lee, "A state-space model for the comprehensive dynamic analysis of three-level voltage-source inverters," 28th Annual IEEE Power Electronics Specialist Conference, pp. 942-948, vol. 2, 1997.

[58] J. Liu, J. Hu, L. Xu, "A modified space vector PWM for Z-source invertermodeling and design," Proceedings of $8^{\text {th }}$ International Conference on Electrical Machines and Systems, vol. 2, pp. 1242-1247, 2005.

[59] N. Kroutikova, C.A. Hernadez-Arambro, T.C. Green, "State-space model of gridconnected inverters under current control mode," IET Electric Power Applications, vol. 1, no. 3, pp. 329-338, 2007.

[60] J. Sun, "Small-signal methods for ac distributed power system-a review," IEEE Transactions on Power Electronics, vol. 24, no. 11, pp. 2545-2554, Nov. 2009.

[61] J. Hu, Z.Q. Zhu, "Investigation on switching patterns of direct power control strategies for grid-connected dc-ac converters based on power variation rates," IEEE Transactions on Power Electronics, vol. 26, no. 12, pp. 3582-3598, Dec. 2011.

[62] Y. Zhang, Z. Jiang, X. Yu, "Small-signal modeling and analysis of parallelconnected voltage source inverters," 6th IEEE International Power Electronics and Motion Control Conference, pp. 377-383, 2009.

[63] N. Pogaku, M. Prodanovic, T.C. Green, "Modeling, analysis and testing of autonomous operation of an inverter-based microgrid", IEEE Transactions on Power electronics, vol. 22, no. 2, pp. 613-625, Mar. 2007.

[64] www.bp.com

[65] www.bp.com/liveassets/bp_internet/solar/bp_solar_usa/STAGING/local_assets/ downloads_pdfs/ BP_175B_datasheet_10_06.pdf

[66] J. Vassallo, J.C Clare, P.W. Wheeler, "A power-equalized harmonic-elimination scheme for utility-connected cascaded H-bridge multilevel converters," 29th Annual IEEE Conference on Industrial Electronics Society, vol. 2, pp. 1185-1190, 2003.

[67] www.ecosolargen.com/wp-content/uploads/2010/08/BP-3110-BP3135.pdf 
[68] www.bp.com/liveassets/bp_internet/bp_korea/bp_korea_english/STAGING/local _assets/downloads_pdfs/a/BP_3230T_10_4087US-2_01_10.pdf

[69] K. Ogata, Modern Control Engineering, Prentice Hall, Fourth edition, Upper Saddle River NJ, USA, 2002.

[70] H.F. Latorre, M. Ghandhari, L. Soder, "Application of control Lyapunov functions to voltage source converters-based high voltage direct current for improving transient stability," Power Tech, 2007 IEEE Lausanne, pp.244-249.

[71] K. Acharya, S.K. Mazumder, I. Basu, "Reaching criterion of a three-phase voltage-source inverter operating with passive and nonlinear loads and its impact on global stability," IEEE Transactions on Industrial Electronics, vol. 55, no. 4, pp.1795-1812, Apr. 2008.

[72] F.S. Garcia, J.A. Pomilio, G.S. Deaecto, J.C. Geromel, "Analysis and control of dc-dc converters based on Lyapunov stability theory," IEEE Energy Conversion Congress and Exposition (ECCE 2009), pp. 2920-2927, 2009.

[73] F. Umbria, J. Aracil, F. Gordillo, "Singular perturbation stability analysis of three phase two-level power converters," $18^{\text {th }}$ Mediterranean Conference on Control \& Automation, pp. 123-128, 2010.

[74] J. Sun, Impedance-based stability criterion for grid-connected inverters," IEEE Transactions on Power Electronics, vol. 26, no. 11, pp. 3075-3078, Nov. 2011.

[75] C. Gezgin, W.C. Bowman, V.J. Thottuvelil, "A stability assessment tool for dc-dc converters," $17^{\text {th }}$ Annual IEEE Applied Power Electronics Conference and Exposition (APEC 2002), vol. 1, pp. 367-373, 2002.

[76] C. Gezgin, "A transient prediction and stability analysis tool for dc-dc converters," $18^{\text {th }}$ Annual IEEE Applied Power Electronics Conference and Exposition (APEC 2003), vol. 2, pp. 1014-1020, 2003.

[77] J. Segundo-Ramirez, E. Barcenas, A. Medina, V. Cardenas, "Steady-state and dynamic state-space model for fast and efficient solution and stability assessment of ASD," IEEE Transactions on Industrial Electronics, vol. 58, no. 7, pp. 28362847, July 2011.

[78] W. Yaunbin, "Research on stability of PWM controlled dc-dc converters in CCM," Second International Conference on Computer Modeling and Simulation, vol. 3, pp. 223-225, 2010.

[79] E. Figueres, G. Garcera, J. Sandia, F. Gonzalez-Espin, J.C. Rubio, "Sensitivity study of the dynamics of three-phase photovoltaic inverters with and LCL grid 
filter," IEEE Transactions on Inductrial Electronics, vol. 56, no. 3, pp. 706-717, Mar. 2009.

[80] L. Yang, Z. Xu, J. Ostergaard, Z.Y. Dong, K.P. Wong, X. Ma, "Oscillatory stability and eigenvalue sensitivity analysis of a DFIG wind turbine system," IEEE Transactions on Energy Conversion, vol. 26, no. 1, pp. 328-339, Mar. 2011.

[81] http://ixdev.ixys.com/DataSheet/1185.pdf

[82] http://www.st.com/internet/com/TECHNICAL_RESOURCES/TECHNICAL _LITERATURE/ DATASHEET/CD00222640.pdf

[83] W. L. Brogan, Modern Control Theory, Prentice Hall, 3rd Edition, 1991.

[84] Z. Yu, A. Mohammed, and I. Panahi, "A review of three PWM techniques," Proceedings of American Control Conference, pp. 257-261, Jun. 1997.

[85] A. Hava, R. Kerkman, and T.A. Lipo, "Carrier-based PWM-VSI overmodulation strategies: analysis, comparison, and design," IEEE Transactions on Power Electronics, vol. 13, no. 4, pp. 674-689, Jul. 1998.

[86] D. G. Holmes and T. A. Lipo, Pulse Width Modulation for Power Converters, Principles and Practice. Hoboken, NJ: IEEE Press, 2003.

[87] J. R.Wells, B. M. Nee, P. L. Chapman, and P. T. Krein, "Selective harmonic control: a general problem formulation and selected solutions," IEEE Transactions on Power Electronics, vol. 20, no. 6, pp. 1337-1345, Nov. 2005.

[88] Mohammed H. Rashid, Power Electronics, Prentice-Hall of India Private Limited, Second Edition, 1994(Book).

[89] Bimal K. Bose, Modern Power Electronics and AC Drives, Pearson education, Second Edition, 2003 (Book).

[90] H.W. van der Broek, H.C. Skudelny, G. Stanke, "Analysis and realization of a pulsewidth modulator based on voltage space vectors", IEEE Transactions on Industry Applications, vol. 24, no. 1, pp. 142-150, Jan./Feb. 1988.

[91] F.Z. Peng, J.S. Lai, "Multilevel converters-a new breed of power converters," IEEE Transactions on Industry Applications, vol. 32, no. 3, pp. 509-517, May/Jun. 1966.

[92] A. Nabae, I. Takahashi, H. Akagi, "A new neutral-point-clamped PWM inverter," IEEE Transactions on Industry Applications, vol. IA-17, no. 5, pp. 518-523, Sep./Oct. 1981. 
[93] P.M. Bhagwat, V.R. Stefanovic, "Generalized structure of a multilevel PWM inverter," IEEE Transactions on Inductry Applications, vol. IA-17, no. 6, pp. 1057-1069, Nov./Dec. 1983.

[94] T.A. Meynard, H. Foch, "Multi-level conversion: high voltage choppers and voltage-source inverters," $23^{\text {rd }}$ Annual IEEE Power Electronics Specialists Conference, vol. 1, pp. 397-403, 1992.

[95] J. Rodriguez, J.S. Lai, F.Z. Peng, "Multilevel inverters: survey of topologies, control, and applications," IEEE Transactions on Industry Applications, vol. 49, no. 4, pp. 724-738, Aug. 2002.

[96] L.M. Tolbert, F.Z. Peng, T. Habetler, "Multilevel converters for large electric drives," IEEE Transactions on Industry Applications, vol. 35, pp. 36-44, Jan./Feb. 1999.

[97] R.H. Park, "Two-reaction theory of synchronous machines-generalized method of analysis, part I," AIEE Transactions, vol. 48, pp. 716-727, Jul. 1929. 


\section{APPENDICES}

The Appendices provided a review of the averaging theory [29] and the perturbation and linearization method [83].

\section{A Averaging Theory}

Equation (A.1) is the general form of the equations, describing a nonlinear dynamical system.

$\dot{\boldsymbol{x}}=\boldsymbol{f}(t, \boldsymbol{x} ; \epsilon)$

where, $\boldsymbol{x}\left(\boldsymbol{x} \in D \subset \mathbb{R}^{n}\right.$, with $D$ an open, bounded set) and $\boldsymbol{f}$ are vectors, elements of $\mathbb{R}^{n}$. The variable $t \in \mathbb{R}$ is usually identified with time; it is assumed $t \geq 0$ or $t \geq t_{0}$ with $t_{0}$ a constant. The parameter $\epsilon$ plays the part of a small parameter which characterizes the magnitude of certain perturbations $\epsilon$. should be taken to be a positive; $0<\epsilon \leq \epsilon_{0}$ with $\epsilon_{0}$ a constant, however, during the approximation process, the limiting value $\epsilon \rightarrow 0$ may be included. All quantities used will be real except if explicitly stated otherwise.

\section{A.1 Definition of Lipschitz Condition}

Consider the vector function $\boldsymbol{f}(t, \boldsymbol{x} ; \epsilon), \boldsymbol{f} \in \mathbb{R}^{n}, t_{0}<t \leq t_{0}+T, \boldsymbol{x} \in D \subset \mathbb{R}^{n}$, $0<\epsilon \leq \epsilon_{0} ; \boldsymbol{f}$ satisfies a Lipschitz condition in $\boldsymbol{x}$ with Lipschitz constant $L$ if in $\left[t_{0}, t_{0}+\right.$ $T] \times D \times\left(0, \epsilon_{0}\right]$

$$
\left\|\boldsymbol{f}\left(t, \boldsymbol{x}_{1} ; \epsilon\right)-\boldsymbol{f}\left(t, \boldsymbol{x}_{2} ; \epsilon\right)\right\| \leq L\left\|\boldsymbol{x}_{1}-\boldsymbol{x}_{\mathbf{2}}\right\|
$$

where $\boldsymbol{x}_{1}, \boldsymbol{x}_{\mathbf{2}} \in D, L$ a constant. Also for a vector $\boldsymbol{u} \in \mathbb{R}^{n}$ with components $u_{i}, \|$. $\|$ indicates the norm 
$\|\boldsymbol{u}\|=\sum_{i=1}^{n}\left|u_{i}\right|$

\section{A.2 Theorem of Existence and Uniqueness}

Consider the initial value problem

$\dot{x}=f(t, x ; \epsilon), x\left(t_{0}\right)=x_{0}$

where $\boldsymbol{x} \in D \subset \mathbb{R}^{n}, \quad t_{0}<t \leq t_{0}+T, \quad 0<\epsilon \leq \epsilon_{0} ; D=\left\{\boldsymbol{x} \mid\left\|\boldsymbol{x}-\boldsymbol{x}_{\mathbf{0}}\right\| \leq d\right\}$. If it is assumed that:

$$
\begin{aligned}
& \boldsymbol{f}(t, \boldsymbol{x} ; \epsilon) \text { is continuous with respect to } t, \boldsymbol{x} ; \epsilon \text { in } G=\left[t_{0}, t_{0}+T\right] \times D \times\left(0, \epsilon_{0}\right] . \\
& \boldsymbol{f}(t, \boldsymbol{x} ; \epsilon) \text { satisfies a Lipschitz condition in } \boldsymbol{x}
\end{aligned}
$$

Then the initial value problem has a unique solution which exits for $t_{0}<t \leq t_{0}+$ $\inf \left(T, \frac{d}{M}\right)$, where $M=\sup _{G}\|\boldsymbol{f}\|$.

\section{A.3 Standard Form Equation}

The equations are often met in the so called standard form

$$
\dot{\boldsymbol{x}}=\epsilon \boldsymbol{f}(t, \boldsymbol{x}), \boldsymbol{x}\left(t_{0}\right)=\boldsymbol{x}_{\mathbf{0}}
$$

Here, if the conditions of the existence and uniqueness theorem have been satisfied, the solution exists for $t_{0}<t \leq t_{0}+\inf \left(T, \frac{d}{M}\right)$ with

$M=\epsilon \sup _{x \in D, t \in\left[t_{0}, t_{0}+T\right)}\|\boldsymbol{f}\|$

Allowing $T$ to be as large as possible, this means that the size of the interval of existence of the solution is of the order of $L / \epsilon$ with $L$ a constant. 


\section{A.4. Definition of Order Function}

A function $\delta(\epsilon)$ is called an order function if $\delta(\epsilon)$ is continuous and positive (or negative) in $(0, \epsilon]$ and if $\lim _{\epsilon \rightarrow 0} \delta(\epsilon)$ exists. Following symbols are used to compare order functions:

$\delta_{1}(\epsilon)=O\left(\delta_{2}(\epsilon)\right)$ for $\epsilon \rightarrow 0$ if there exist a constant $k$ such that $\left|\delta_{1}(\epsilon)\right| \leq$ $k\left|\delta_{2}(\epsilon)\right|$ for $\epsilon \rightarrow 0$.

$\delta_{1}(\epsilon)=o\left(\delta_{2}(\epsilon)\right)$ for $\epsilon \rightarrow 0$ if $\lim _{\epsilon \rightarrow 0} \frac{\delta_{1}(\epsilon)}{\delta_{2}(\epsilon)}=0$. Note that, $\delta_{1}(\epsilon)=o\left(\delta_{2}(\epsilon)\right)$ implies that $\delta_{1}(\epsilon)=O\left(\delta_{2}(\epsilon)\right)$.

$\delta_{1}(\epsilon)=O_{S}\left(\delta_{2}(\epsilon)\right)$ for $\epsilon \rightarrow 0$ if $\delta_{1}(\epsilon)=O\left(\delta_{2}(\epsilon)\right)$ and $\delta_{1}(\epsilon) \neq o\left(\delta_{2}(\epsilon)\right)$.

A.5 Definition of Order of Magnitude of $\phi_{\epsilon}$ in $I$

$\phi_{\epsilon}=O(\delta(\epsilon))$ in $I$ if there exists a constant $k$ such that $\left\|\phi_{\epsilon}\right\|=O(\delta(\epsilon))$ for $\epsilon \rightarrow 0, \delta(\epsilon)$ an order function on $(0, \epsilon]$ and $\|$.$\| a norm for \phi$ as a function of $t$.

$$
\begin{aligned}
& \phi_{\epsilon}=o(\delta(\epsilon)) \text { in } I \text { if } \lim _{\epsilon \rightarrow 0} \frac{\left\|\phi_{\epsilon}\right\|}{\delta(\epsilon)}=0 . \\
& \phi_{\epsilon}=O_{S}(\delta(\epsilon)) \text { in } I \text { if } \phi_{\epsilon}=O(\delta(\epsilon)) \text { and } \phi_{\epsilon} \neq o(\delta(\epsilon)) .
\end{aligned}
$$

Note that this definition implies that the norm of a function is allowed to be $\epsilon$-dependent. One should realize that $\left\|\phi_{\epsilon}\right\|=\sup _{t \in I}\left\|\phi_{\epsilon}(t)\right\|$. 


\section{A.6 Definition of Time-Scale}

$\phi_{\epsilon}=O\left(\delta_{0}(\epsilon)\right)$ as $\epsilon \rightarrow 0$ on the time-scale $\delta^{-1}(\epsilon)$ if the estimate holds for $0 \leq \delta(\epsilon) t \leq L$ with $L$ a constant independent of $\epsilon$. An analogous definition can be given for $o\left(\delta_{0}(\epsilon)\right)$-estimates.

\section{A.7 Theorem of First Order Averaging}

Consider the initial value problems

$\dot{\boldsymbol{x}}=\epsilon \boldsymbol{f}(t, \boldsymbol{x})+\epsilon^{2} \boldsymbol{g}(t, \boldsymbol{x}, \epsilon), \boldsymbol{x}\left(t_{0}\right)=\boldsymbol{x}_{\mathbf{0}}$

and

$\dot{\boldsymbol{y}}=\epsilon \boldsymbol{F}(\boldsymbol{y}), \boldsymbol{y}\left(t_{0}\right)=\boldsymbol{x}_{\mathbf{0}}$

where

$\boldsymbol{F}(\boldsymbol{x})=\frac{1}{T} \int_{0}^{T} \boldsymbol{f}(t, \boldsymbol{x}) d t$

with $\boldsymbol{x}, \boldsymbol{y}, \boldsymbol{x}_{\mathbf{0}} \in D \subset \mathbb{R}^{n}, t \in\left[t_{0}, \infty\right), \epsilon \in\left(0, \epsilon_{0}\right]$. Suppose:

$\boldsymbol{f}, \boldsymbol{g}$ and $\boldsymbol{\nabla} \boldsymbol{f}$ (where $\boldsymbol{\nabla} \boldsymbol{f}$ indicates the derivative of $\boldsymbol{f}$ with respect to the spatial variable $\boldsymbol{x}$ ) are defined, continuous and bounded by a constant $M$ independent of $\epsilon$, in $\left[t_{0}, \infty\right) \times D$

$\boldsymbol{g}$ is Lipschitz-continuous with respect to $x \in D$;

$\boldsymbol{f}$ is $T$-periodic in $t$ with $T$ a constant, independent of $\epsilon$;

$\boldsymbol{y}(t)$ belongs to an ( $\epsilon$-independent) interior subset of $D$ on the time-scale $1 / \epsilon$;

then $\boldsymbol{x}(t)-\boldsymbol{y}(t)=\boldsymbol{O}(\epsilon)$ on the time-scale $\frac{1}{\epsilon}$. 


\section{A.8 Definition of Local Average}

Consider the continuous vectorfield $\boldsymbol{f}: \mathbb{R} \times \mathbb{R}^{p} \rightarrow \mathbb{R}^{n}$. We define the local average $\boldsymbol{f}_{\boldsymbol{T}}$ of $\boldsymbol{f}$ by

$\boldsymbol{f}_{\boldsymbol{T}}(t, \boldsymbol{x})=\frac{1}{T} \int_{0}^{T} \boldsymbol{f}(t+\tau, \boldsymbol{x}) d \tau$

in which $\tau$ is a dummy variable; $p$ is zero or a natural number, e.g. $n$. Also, if $\boldsymbol{f}$ is Tperiodic in $t$. Then

$\boldsymbol{f}_{\boldsymbol{T}}(t, \boldsymbol{x})=\boldsymbol{F}(\boldsymbol{x})=\frac{1}{T} \int_{0}^{T} \boldsymbol{f}(t, \boldsymbol{x}) d t$

\section{A.9 Lemma (Local Average Approximation)}

Consider the initial value problem (A.5)

$\dot{\boldsymbol{x}}=\epsilon \boldsymbol{f}(t, \boldsymbol{x}), \boldsymbol{x}\left(t_{0}\right)=x_{\mathbf{0}}$

with $\boldsymbol{f}: \mathbb{R} \times \mathbb{R}^{n} \rightarrow \mathbb{R}^{n}$, Lipschitz-continuous in $\boldsymbol{x}$ on $D \subset \mathbb{R}^{n}, t$ on the time-scale $\frac{1}{\epsilon} ; \boldsymbol{f}$ continuous in $t$ and $\boldsymbol{x}$. If $\boldsymbol{y}$ is the solution of (A.12)

$\dot{\boldsymbol{y}}=\epsilon \boldsymbol{f}_{T}(t, \boldsymbol{y}), \boldsymbol{y}\left(t_{0}\right)=\boldsymbol{x}_{\mathbf{0}}$

then, $\boldsymbol{x}(t)-\boldsymbol{y}(t)=\boldsymbol{O}(\epsilon)$ as $\epsilon \rightarrow 0$ on the time-scale $\frac{1}{\epsilon}$.

\section{A.10 Theorem of Periodic Averaging}

Consider the initial value problem (A.5)

$\dot{\boldsymbol{x}}=\epsilon \boldsymbol{f}(t, \boldsymbol{x}), \boldsymbol{x}\left(t_{0}\right)=\boldsymbol{x}_{\mathbf{0}}$

with $\boldsymbol{f}: \mathbb{R}^{n+1} \rightarrow \mathbb{R}^{n}$ and (A.8)

$\dot{\boldsymbol{y}}=\epsilon \boldsymbol{F}(\boldsymbol{y}), \boldsymbol{y}\left(t_{0}\right)=\boldsymbol{x}_{\mathbf{0}}$

$\boldsymbol{x}, \boldsymbol{y}, \boldsymbol{x}_{\mathbf{0}} \in D \subset \mathbb{R}^{n}, t \in[t, \infty), \epsilon \in\left(0, \epsilon_{0}\right]$. Suppose 
$f$ has period $T$;

$\boldsymbol{f}$ is Lipschitz-continuous in $\boldsymbol{x}$ on $D \subset \mathbb{R}^{n}, t \geq 0$, continuous in $t$ and $\boldsymbol{x}$ on $\mathbb{R}^{+} \times D$ and with average $\boldsymbol{F}$;

$\boldsymbol{y}(t)$ belongs to an interior subset of $D$ on the time-scale $\frac{1}{\epsilon}$;

then

$\boldsymbol{x}(t)-\boldsymbol{y}(t)=\boldsymbol{O}(\epsilon)$ as $\epsilon \rightarrow 0$ on the time-scale $\frac{1}{\epsilon}$.

\section{A.11 Definition of KBM-Vectorfield}

Consider the vectorfield $f(t, x)$ with $\boldsymbol{f}: \mathbb{R} \times \mathbb{R}^{n} \rightarrow \mathbb{R}^{n}$, Lipschitz-continuous in $\boldsymbol{x}$ on $D \subset \mathbb{R}^{n}, t \geq 0 ; \boldsymbol{f}$ continuous in $t$ and $\boldsymbol{x}$ on $\mathbb{R}^{+} \times D$. If the average

$\boldsymbol{F}(\boldsymbol{x})=\lim _{T \rightarrow \infty} \frac{1}{T} \int_{0}^{T} \boldsymbol{f}(t, \boldsymbol{x}) d t$

exists, $\boldsymbol{f}$ is called a KBM-vectorfield (KBM stands for Krylov, Bogoliubov and Mitropolsky).

\section{A.12 Lemma (Approximating Local Average with the Usual Average)}

Let $y$ be the solution of the initial value problem (A.12)

$\dot{\boldsymbol{y}}=\epsilon \boldsymbol{f}_{T}(t, \boldsymbol{y}), \boldsymbol{y}\left(t_{0}\right)=\boldsymbol{x}_{\mathbf{0}}$

We suppose $\boldsymbol{f}$ is a KBM-vectorfield; $\mathbf{z}$ is the solution of the initial value problem

$\dot{\mathbf{z}}=\epsilon \boldsymbol{F}(\mathbf{z}), \mathbf{z}\left(t_{0}\right)=\boldsymbol{x}_{\mathbf{0}}$

then

$\boldsymbol{x}(t)-\boldsymbol{y}(t)=\boldsymbol{O}\left(\frac{\delta(\epsilon)}{T \epsilon}\right)$ 
with $t$ on the time-scale $\frac{1}{\epsilon}$.

\section{A.13 Theorem of General Averaging}

Consider the initial value problem (A.5)

$\dot{\boldsymbol{x}}=\epsilon \boldsymbol{f}(t, \boldsymbol{x}), \boldsymbol{x}\left(t_{0}\right)=x_{0}$

with $\boldsymbol{f}: \mathbb{R}^{n+1} \rightarrow \mathbb{R}^{n}$ and (A.8)

$\dot{\boldsymbol{y}}=\epsilon \boldsymbol{F}(\boldsymbol{y}), \boldsymbol{y}\left(t_{0}\right)=\boldsymbol{x}_{\mathbf{0}}$

$\boldsymbol{x}, \boldsymbol{y}, \boldsymbol{x}_{\mathbf{0}} \in D \subset \mathbb{R}^{n}, t \in[t, \infty), \epsilon \in\left(0, \epsilon_{0}\right]$. Suppose

$\boldsymbol{f}$ is a KBM-vectorfield with average $\boldsymbol{F}$;

$\boldsymbol{y}(t)$ belongs to an interior subset of $D$ on the time-scale $\frac{1}{\epsilon}$;

then

$\boldsymbol{x}(t)-\boldsymbol{y}(t)=\boldsymbol{O}\left(\delta^{1 / 2}(\epsilon)\right)$ as $\epsilon \rightarrow 0$ on the time-scale $\frac{1}{\epsilon}$

where,

$\delta(\epsilon)=\sup _{\boldsymbol{x} \in D} \sup _{t \in\left[0, \frac{L}{\epsilon}\right)} \epsilon\left|\int_{0}^{t}[\boldsymbol{f}(\tau, \boldsymbol{x})-\boldsymbol{F}(\boldsymbol{x})] d \tau\right|$

\section{B Perturbation and Linearization Method}

Let represent a nonlinear system by

$$
\begin{aligned}
& \dot{\boldsymbol{x}}(t)=\boldsymbol{f}(\boldsymbol{x}(t), \boldsymbol{u}(t)) \\
& \boldsymbol{y}(t)=\boldsymbol{h}(\boldsymbol{x}(t), \boldsymbol{u}(t))
\end{aligned}
$$

If $\boldsymbol{x}(t), \boldsymbol{y}(t)$, and $\boldsymbol{u}(t)$ are perturbed about their steady-state values, $\boldsymbol{X}(t), \boldsymbol{Y}(t)$, and $\boldsymbol{U}(t)$, as: 


$$
\begin{aligned}
\boldsymbol{x}(t) & =\boldsymbol{X}(t)+\boldsymbol{\delta} \boldsymbol{x}(t) \\
\boldsymbol{u}(t) & =\boldsymbol{U}(t)+\boldsymbol{\delta} \boldsymbol{u}(t) \\
\boldsymbol{y}(t) & =\boldsymbol{Y}(t)+\boldsymbol{\delta} \boldsymbol{y}(t)
\end{aligned}
$$

where,

$$
\begin{aligned}
& \dot{\boldsymbol{X}}(t)=\boldsymbol{f}(\boldsymbol{X}(t), \boldsymbol{U}(t)) \\
& \boldsymbol{Y}(t)=\boldsymbol{h}(\boldsymbol{X}(t), \boldsymbol{U}(t))
\end{aligned}
$$

Substituting the perturbation into the nonlinear system equations provides

$$
\begin{aligned}
& \dot{\boldsymbol{X}}(t)+\boldsymbol{\delta} \dot{\boldsymbol{x}}(t)=\boldsymbol{f}(\boldsymbol{X}(t)+\boldsymbol{\delta} \boldsymbol{x}(t), \boldsymbol{U}(t)+\boldsymbol{\delta} \boldsymbol{u}(t)) \\
& \boldsymbol{Y}(t)+\boldsymbol{\delta} \boldsymbol{y}(t)=\boldsymbol{h}(\boldsymbol{X}(t)+\boldsymbol{\delta} \boldsymbol{x}(t), \boldsymbol{U}(t)+\boldsymbol{\delta} \boldsymbol{u}(t))
\end{aligned}
$$

Expanding the components of $f$ and $\boldsymbol{h}$ by Taylor series yields

$$
\begin{aligned}
\dot{\boldsymbol{X}}(t)+\boldsymbol{\delta} \dot{\boldsymbol{x}}(t) & =\boldsymbol{f}(\boldsymbol{X}(t), \boldsymbol{U}(t))+\left[\frac{\partial f}{\partial \boldsymbol{x}}\right]_{\boldsymbol{X}} \boldsymbol{\delta} \boldsymbol{x}+\left[\frac{\partial f}{\partial \boldsymbol{u}}\right]_{\boldsymbol{U}} \boldsymbol{\delta} \boldsymbol{u}+\text { h.o.t } \\
\boldsymbol{Y}(t)+\boldsymbol{\delta} \boldsymbol{y}(t) & =\boldsymbol{h}(\boldsymbol{X}(t), \boldsymbol{U}(t))+\left[\frac{\partial \boldsymbol{h}}{\partial \boldsymbol{x}}\right]_{\boldsymbol{X}} \boldsymbol{\delta} \boldsymbol{x}+\left[\frac{\partial \boldsymbol{h}}{\partial \boldsymbol{u}}\right]_{\boldsymbol{U}} \boldsymbol{\delta} \boldsymbol{u}+\text { h.o.t }
\end{aligned}
$$

which, neglecting the higher order terms (h.o.t), gives

$$
\begin{aligned}
\delta \dot{x}(t) & \cong\left[\frac{\partial f}{\partial x}\right]_{X} \delta \boldsymbol{x}+\left[\frac{\partial f}{\partial u}\right]_{U} \delta \boldsymbol{u} \\
\delta \boldsymbol{y}(t) & \cong\left[\frac{\partial h}{\partial x}\right]_{X} \delta \boldsymbol{x}+\left[\frac{\partial h}{\partial u}\right]_{U} \delta \boldsymbol{u}
\end{aligned}
$$

Let define

$$
\begin{aligned}
\boldsymbol{A} & =\left[\frac{\partial f}{\partial x}\right]_{X}, \text { and } & \boldsymbol{B} & =\left[\frac{\partial f}{\partial u}\right]_{U} \\
\boldsymbol{C} & =\left[\frac{\partial \boldsymbol{h}}{\partial x}\right]_{X}, \text { and } & \boldsymbol{D} & =\left[\frac{\partial \boldsymbol{h}}{\partial \boldsymbol{u}}\right]_{U}
\end{aligned}
$$

then, the linearized system model can described by

$$
\begin{aligned}
& \boldsymbol{\delta} \dot{\boldsymbol{x}}(t)=\boldsymbol{A} \boldsymbol{\delta} \boldsymbol{x}+\boldsymbol{B} \boldsymbol{\delta} \boldsymbol{u} \\
& \boldsymbol{\delta} \boldsymbol{y}(t)=\boldsymbol{C} \boldsymbol{\delta} \boldsymbol{x}+\boldsymbol{D} \boldsymbol{\delta} \boldsymbol{u}
\end{aligned}
$$

where, 


$$
\begin{array}{rlrl}
a_{i j} & =\left[\frac{\partial f_{i}}{\partial x_{j}}\right]_{X_{j}}, \text { and } & b_{i j} & =\left[\frac{\partial f_{i}}{\partial u_{j}}\right]_{U_{j}} \\
c_{i j} & =\left[\frac{\partial h_{i}}{\partial x_{j}}\right]_{X_{j}}, \text { and } & d_{i j}=\left[\frac{\partial h_{i}}{\partial u_{j}}\right]_{U_{j}}
\end{array}
$$




\section{ALI KASHEFI KAVIANI}

June 20, 1982

2000-2005

2005-2008

2009-2012
Born, Gorgan, Iran

B.Sc., Electrical Engineering

K.N. Toosi University of Technology

Tehran, Iran

M.Sc., Electrical Engineering

Amirkabir University of Technology (Tehran Polytechnic)

Tehran, Iran

PH.D. Candidate, Electrical Engineering

Florida International University

Miami, Florida

\section{PUBLICATIONS AND PRESENTATIONS}

\section{SELECTED JOURNALS}

[1] A.K. Kaviani, B. Hadley, B. Mirafzal, "A Time-Coordination Approach for Regenerative Energy Saving in Multi-Axis Motor-Drive Systems," IEEE Transaction on Power Electronics, vol. 27, no. 2, February 2012, pp. 931-941.

[2] A.K. Kaviani, G.H. Riahy, SH.M. Kouhsari, "Optimal Design of a Reliable Hydrogen-based Stand-alone Wind/PV Generating System, Considering Components Outages," Renewable Energy, Volume 34, Issue 11, November 2009, Pages 2380-2390.

[3] A.K. Kaviani, H.R. Baghaee, G.H. Riahy, "Optimal Sizing of a Stand-alone Wind/Photovoltaic Generation Unit using Particle Swarm Optimization," SIMULATION-Transactions of the Society for Modeling and Simulation International, Vol. 85, Issue 2, February 2009, pp. 89-89.

[4] M.T. Bina, A.K. Kaviani, "Three-Phase Unbalance of Distribution Systems: Complementary Analysis and Experimental Case Study," International Journal of Electrical Power \& Energy Systems, Volume 33, Issue 4, May 2011, Pages 817826.

[5] B. Mirafzal, M. Saghaleini, A.K. Kaviani, "An SVPWM-based Switching Pattern for Stand-Alone and Grid-Connected Three-Phase Single-Stage Boost-Inverters," 
IEEE Transaction on Power Electronics, vol. 26, no. 4, April 2011, pp. $1102-$ 1111.

\section{SELECTED CONFERENCES}

[1] A.K. Kaviani, B. Mirafzal, "Dynamic Model of the Three-Phase Single-Stage Boost Inverter, for Grid-Connected Applications," Accepted in 2012 IEEE Energy Conversion and Exposition (ECCE 2012), Sep. 16-20, 2012.

[2] A.K. Kaviani, B. Mirafzal, "Grid-Connected to Stand-Alone Transition of the Three-Phase Single-Stage Boost Inverter," Accepted in 2012 IEEE Energy Conversion and Exposition (ECCE 2012), Sep. 16-20, 2012.

[3] A.K. Kaviani, K. Yen, "Active Power Control in a Grid Connected Distributed Generation System via Time Delay Method," Accepted in 12th Power and Energy Society General Meeting (PES 2012), July 22-26, 2012.

[4] A.K. Kaviani, B. Mirafzal, K. Yen, "Dynamic Model of Three-Phase CurrentSource Boost-Inverter for Stand-Alone Applications," 27th Annual IEEE Applied Power Electronics Conference \& Exposition (APEC 2012), pp. 218-224.

[5] A.K. Kaviani, B. Mirafzal, "A Switching Pattern for Single-Phase Single-Stage Current-Source Boost-Inverter," 27th Annual IEEE Applied Power Electronics Conference \& Exposition (APEC 2012), pp. 2066-2071.

[6] A.K. Kaviani, B. Hadley, B. Mirafzal, "A Time-Coordination Approach for Regenerative Energy Saving in Multi-Axis Motor-Drive Systems," 2011 IEEE Energy Conversion and Exposition (ECCE 2011), pp. 3450-3457.

[7] A.K. Kaviani, M. Elshaer, O.A. Mohammed, "Enhancing Loading Limitations in PV Systems," 36th Annual Conference on IEEE Industrial Electronics Society, IECON 2010, pp. 2790-2794.

\section{AWARDS AND ACHIEVEMENTS}

- $\quad$ Recipient of Dissertation Year Fellowship Award, 2011 University Graduate School, Florida Int. University

- $\quad$ Elite Graduate Member of National Elite Organization of Iran, since 2008

- $\quad$ Honored as the First Rank amongst 7 Graduate Students, 2008 Department of Electrical Engineering, Amirkabir University of Technology

- $\quad$ Honored as the Second Rank amongst 35 Undergraduate Students, 2005 Department of Electrical Engineering, K.N. Toosi University of Technology 